\title{
Game Theoretic ApProach to Medium ACCESS CONTROL IN Wireless NETWORKS
}

\author{
by \\ Seyed Hani Elamahdi Mortazavi Najafabadi
}

A thesis submitted to

The University of Birmingham

for the degree of

Doctor OF PHILOSOPHY

School of Electronic, Electrical and Computer Engineering College of Engineering and Physical Sciences

The University of Birmingham

March 2014 


\section{UNIVERSITYOF BIRMINGHAM}

\section{University of Birmingham Research Archive \\ e-theses repository}

This unpublished thesis/dissertation is copyright of the author and/or third parties. The intellectual property rights of the author or third parties in respect of this work are as defined by The Copyright Designs and Patents Act 1988 or as modified by any successor legislation.

Any use made of information contained in this thesis/dissertation must be in accordance with that legislation and must be properly acknowledged. Further distribution or reproduction in any format is prohibited without the permission of the copyright holder. 


\section{ABstract}

Wireless networking is fast becoming the primary method for people to connect to the Internet and with each other. The available wireless spectrum is increasingly congested, with users demanding higher performance and reliability from their wireless connections. This thesis proposes a game-theoretic random access model, compliant with the IEEE 802.11 standard, that can be integrated into the distributed coordination function (DCF). The objective is to design a game theoretic model that potentially optimizes throughput and fairness in each node independently and, therefore, minimise channel access delay. This dissertation presents a game-theoretic MAC layer implementation for single-cell networks and centralised DCF in the presence of hidden terminals to show how game theory can be applied to improve wireless performance. A utility function is proposed, such that it can decouple the protocol's dynamic adaptation to channel load from collision detection. It is demonstrated that the proposed model can reach a Nash equilibrium that results in a relatively stable contention window, provided that a node adapts its behaviour to the idle rate of the broadcast channel, coupled with observation of its own transmission activity. This dissertation shows that the proposed game-theoretic model is capable of achieving much higher throughput than the standard IEEE 802.11 DCF with better short-time fairness and significant improvements in the channel access delay. 
To my parents and my sister 


\section{ACKNOWLEDGEMENTS}

The endeavour of doctoral research entails many trials and tribulations. My time at Birmingham, through ups and downs, was enriched in a variety of ways with the care and contributions of many people. Costas Constantinou, my supervisor, has been very supportive of me throughout this journey. His attention to detail and focus on obtaining practical results have been formative in all research that I have done since joining his group. I would also like to thank him for instilling in me the importance of good writing and presenting research in a human and understandable form and for all his technical insights during the early part of my Ph.D. His questions about my research always made me think harder, dig deeper, and often come up with better solutions. His enthusiasm for both technical and non-technical aspects of life, especially photography, is infectious. I would like to thank Saeid Safavi and Davood Godarzi for spending many sleepless nights with me while I was trying to get my simulation engine working days before the paper deadlines and for many deep discussions about the notion of life throughout my Ph.D. My stay at Birmingham benefited tremendously from interactions with all members of the Distributed Systems and Networks Laboratory, past and present including Amalia Tsanaka, Paul Kiddie, David Arjona, Debra Topham, and especially Mary Winkles in the postgraduate office for her great help and support. I made some of my best friends after I came to Birmingham while further strengthening several existing friendships. Kasra Daheshpour has been immensely helpful, providing company throughout my Ph.D. in Birmingham, cooking great food, and being a great roommate for a brief period of time. 
I would like to thank Ashkan Izadi, Amir Kasra Asghari, Janos Barberis, and Abulsoud Hanani my Birmingham friends. Finally, I would like to thank my sister Fatemeh and my parents. They supported me when I decided to come to Birmingham without any guaranteed funding and never let me worry about anything other than work during my Ph.D. They are responsible for me never having to experience the life of a poor grad student. My parents have been a constant source of support throughout my life. This thesis is dedicated to them. 


\section{CONTENTS}

$\begin{array}{lll}1 & \text { Introduction } & 1\end{array}$

1.1 Wireless Random Access MAC Protocols Today . . . . . . . . . . . . . . 5

1.2 Game Theory for Wireless MAC . . . . . . . . . . . . . . . . . . . 9 9

1.3 Contributions . . . . . . . . . . . . . . . . 11

1.4 Outline. . . . . . . . . . . . . . . . . . . . . 13

2 Contention-Based Random Channel Access Schemes 14

2.1 Wireless Medium from Random Channel Access Point of View . . . . . . . 14

2.1.1 Single channel Half-duplex . . . . . . . . . . . . . . . 15

2.1 .2 Location-dependent carrier sensing . . . . . . . . . . . . . . . 17

2.1 .3 Time-varying channel . . . . . . . . . . . . . . . . . . 18

2.2 The Design of MAC for Random Channel Access Schemes . . . . . . . . . 18

$2.2 .1 \quad$ Performance analysis of DCF in single-cell wireless networks . . . . 20

2.2 .2 Performance analysis of DCF in the presence of hidden terminals $\quad 22$

2.2 .3 Enhancing contention resolution algorithms . . . . . . . . . 24

2.3 Game Theory and its Application to Random Channel Access Schemes . . 28

2.4 Game Theoretic Models . . . . . . . . . . . . . . . . . . . . . . 29

2.4 .1 Noncooperative games . . . . . . . . . . . . . . . . . 29

2.4 .2 Cooperative games $\ldots \ldots \ldots \ldots \ldots \ldots$

\begin{tabular}{lll}
\hline 2.4 .3 & Challenges in game theoretic design of random channel access schemes 33
\end{tabular}

2.5 Games for Multiple Access Schemes . . . . . . . . . . . . . . . . 34

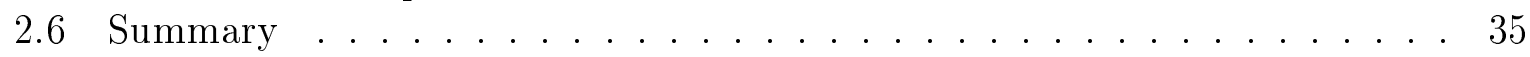

3 Game Theoretic DCF for Single Cell Wireless Network 38

3.1 Introduction . . . . . . . . . . . . . . . . . . 38

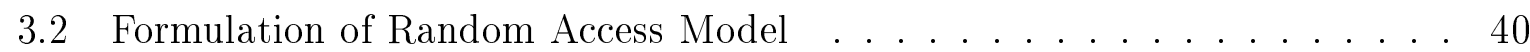

3.2 .1 Description of DCF . . . . . . . . . . . . . . . . . . . 40

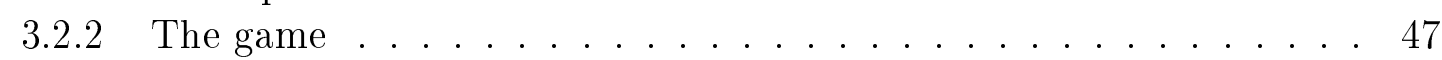

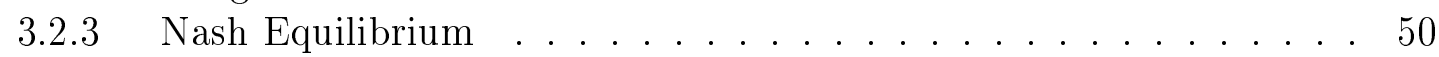

3.2 .4 Utility function design . . . . . . . . . . . . . . . . . . . 51

3.2 .5 Dynamics of random access game . . . . . . . . . . . . . 57

3.3 Medium Access Control Design $\ldots \ldots \ldots \ldots$. . . . . . . . . . 58

3.3.1 Conditional collision probability estimation . . . . . . . . . . 58

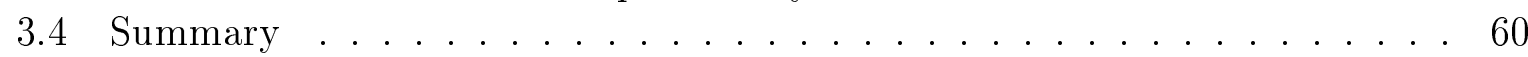


4 Game Theoretic DCF in Presence of the Hidden Terminals 62

4.1 Analysis of Channel Contention . . . . . . . . . . . . . . . 63

4.1 .1 Classic hidden station . . . . . . . . . . . . . . . . . 64

4.1.2 The hidden terminal effect on the DCF (time-based point of view) . 64

4.1.3 Analytical model of the DCF in the presence of the hidden stations 68

4.2 Formulation of Game Theoretic Random Access Model $\ldots \ldots \ldots$. . . 70

$4.2 .1 \quad$ Nash Equilibrium . . . . . . . . . . . . . . . . . . . . 72

4.2 .2 Utility function design $\ldots \ldots \ldots \ldots \ldots . \ldots \ldots$

4.2 .3 Dynamics of random access game $\ldots \ldots \ldots \ldots$. . . . . . 81

4.3 Medium Access Control Design $\ldots \ldots \ldots$. . . . . . . . . . . 83

4.3 .1 Conditional collision probability estimation . . . . . . . . 83

4.4 Summary $\ldots \ldots \ldots \ldots \ldots \ldots \ldots \ldots \ldots \ldots \ldots \ldots$

5 Simulation Methodology 85

5.1 Introduction . . . . . . . . . . . . . . . . . . . 85

5.2 Simulation Environment . . . . . . . . . . . . . . . . . . . . 86

5.3 Simulation Configuration . . . . . . . . . . . . . . . . . 88

$5.3 .1 \quad$ Simulation topology $\ldots \ldots \ldots \ldots$. . . . . . . . . . . 89

5.3 .2 Network modelling . . . . . . . . . . . . . . . . . . . . . . 91

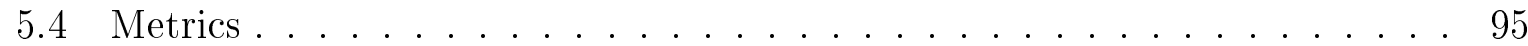

5.4 .1 Throughput . . . . . . . . . . . . . . . . . 96

5.4 .2 Average delay $\ldots \ldots \ldots \ldots \ldots$. . . . . . . . . . . 97

5.4 .3 Collision rate $\ldots \ldots \ldots \ldots \ldots \ldots \ldots$

5.4 .4 Jain index . . . . . . . . . . . . . . . . . . . . . . . 98

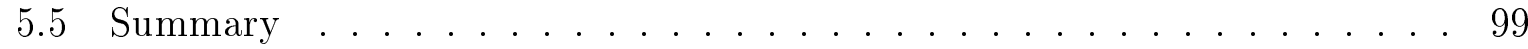

6 Simulation Results 100

6.1 Introduction . . . . . . . . . . . . . . . . . . . 100

6.2 Saturation Throughput and Collision Rate . . . . . . . . . . . . 101

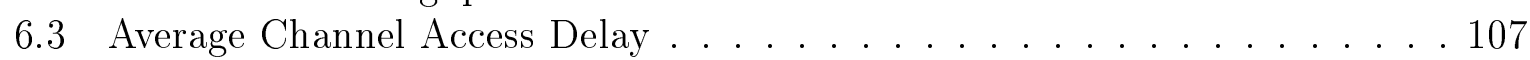

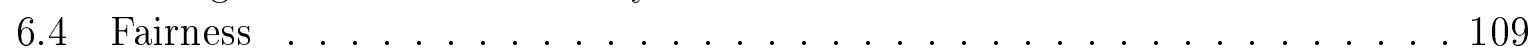

6.5 Conclusion . . . . . . . . . . . . . . . . . . . 111

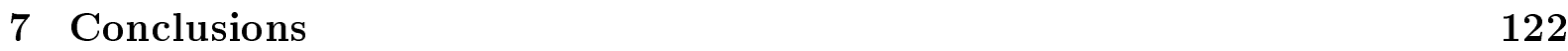

7.1 Future Work . . . . . . . . . . . . . . . . . . . . . . . 123

$\begin{array}{ll}\text { List of References } & 127\end{array}$

\begin{tabular}{|l|l|l|l}
\hline A Derivation of Utility functions & 137
\end{tabular}

A.1 Derevation of Equation $3.2 .19|\&| 3.2 .22 \quad \ldots \ldots \ldots \ldots . \ldots \ldots$

\begin{tabular}{ll}
\hline B Normalised delay distribution & 138
\end{tabular} 
C Common Parameters Used for MiXiM-OmnetPP Simulations 158 C.1 omnetpp.ini . . . . . . . . . . . . . . . . . . . . . 158

\begin{tabular}{ll}
\hline D Publications & 162
\end{tabular} 


\section{LIST OF FIGURES}

1.1 Shows a generic multiple access communication situation in which a number of stations share a transmission medium $(N$ denotes the number of stations

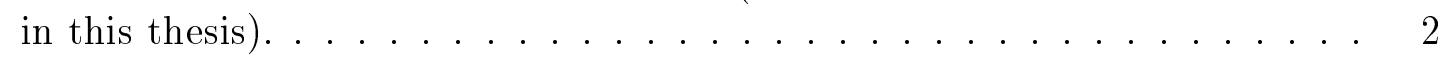

1.2 Approaches to sharing a transmission medium. . . . . . . . . . . . 3

1.3 Typical centralised wireless network, also known as last-hop network. . . . 4

1.4 Typical distributed wireless network, also known as ad-hoc network. . . . . 5

$3.1 \quad$ Backoff timer operation as a part of collision avoidance mechanism in DCF. 41

3.2 Receiving the ACK and CRC check operation. . . . . . . . . . . . . . 42

3.3 Virtual carrier sensing, duration field and the NAV timer. . . . . . . . . . . 43

3.4 Example of basic access mechanism in a single cell wireless network. . . . . 44

3.5 The simplified basic access mechanism operation. . . . . . . . . . . 45

$3.6 \quad$ Example of RTS/CTS mechanism in a single-cell wireless network. . . . . . 46

3.7 Number of nodes versus optimal number of idle slots $\bar{n}_{i}^{\text {opt }}$ for basic access mechanism, up to 50 active nodes based on the parameters in table $3.1 \mid$

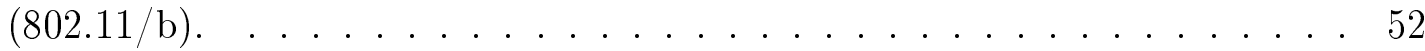

3.8 Number of nodes versus optimal number of idle slots $\bar{n}_{i}^{\text {opt }}$ for RTS/CTS access mechanism, up to 50 active nodes based on the parameters in table

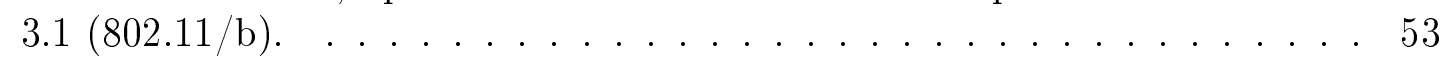

3.9 Number of nodes versus optimal values of contention window size $C W_{\text {opt }}$ for basic access mechanism based on the parameters in table $3.2[(802.11 /$ b). 54

3.10 Number of nodes versus optimal values of contention window size $C W_{\text {opt }}$ for RTS/CTS access mechanism based on the parameters in table $|3.1|(802.11 / \mathrm{b}) .455$

4.1 The classic hidden station . . . . . . . . . . . . . . . . . . . 64

4.2 AP scenario in the presence of hidden nodes from the node $\mathrm{X}$ point of view: The dashed circle represents the carrier sense range of X. The solid black circles represent nodes covered by both $\mathrm{X}$ and the AP, whereas the open circles represent hidden terminals for X but not the AP. . . . . . . . . . 65

4.3 The vulnerable period $\left(\tau_{V}\right)$ : The basic access method. For details of all

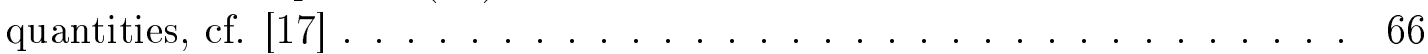

4.4 The vulnerable period $\left(\tau_{V}\right)$ : RTS/CTS access method. For details of all

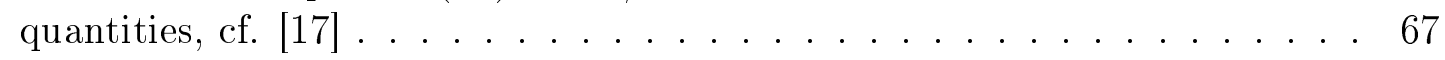


4.5 Number of nodes versus number of hidden nodes from each node point of view versus optimal number of idle slots $\bar{n}_{i}^{\text {opt }}$ for basic access mechanism based on the parameters in Table $3.1[(802.11 /$ b) $. \quad \ldots \ldots . \ldots 78$

4.6 Number of nodes versus number of hidden nodes from each node point of view versus optimal number of idle slots $\bar{n}_{i}^{\text {opt }}$ for RTS/CTS access mechanism based on the parameters in Table $4.1(802.11 / \mathrm{b}) . \quad \ldots \ldots . . \ldots 78$

4.7 Number of nodes versus number of hidden nodes from each node point of view versus optimal values of contention window size $C W_{\text {opt }}$ for basic access

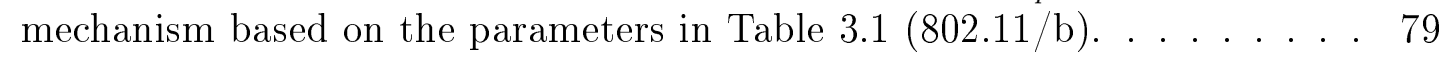

4.8 Number of nodes versus number of hidden nodes from each node point of view versus optimal values of contention window size $C W_{\text {opt }}$ for RTS/CTS

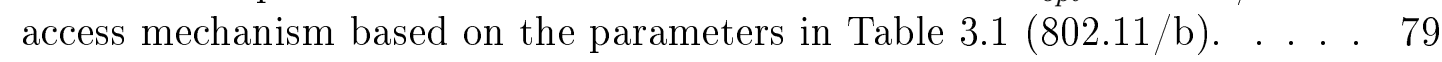

$5.1 \quad$ OSI model vs MiXiM model . . . . . . . . . . . . . . . . . . 87

5.2 Ad hoc network topology with 5 nodes and no hidden terminal. . . . . . . . 90

$5.3 \quad$ Calculation of network with specific hidden node percentage $(\mu) . \quad$. . . . . 90

5.4 An example of centralised topology. A network with 5 nodes and $20 \%$ hidden terminal. . . . . . . . . . . . . . . . . . . 91

5.5 The figure represents the MiXiM node model. The green background box labelled "nic" represents the MAC and phy modules. Arp is used by network layer and MAC layer for address resolution. Arrows connecting the sub modules represent connections and gates. . . . . . . . . . . . 93

5.6 The ACK transmission procedure in event log. . . . . . . . . . . . . . . . . . 94

5.7 Measured throughput with slowly increasing offered load. . . . . . . . . . . 97

6.1 Saturation throughput comparison for network of 5-40 competing active nodes operating in basic access and RTS/CTS mechanism. . . . . . . . . . 102

6.2 Average collision rate comparison for a network of 5-40 active nodes oper-

ating in basic access method. . . . . . . . . . . . . 103

6.3 Average collision rate comparison for a network of 5-40 active nodes operating in RTS/CTS access method. . . . . . . . . . . . . 103

6.4 Saturation throughput comparison for network of 5-40 competing active nodes and with different percentage of hidden terminals, operating in basic access. . . . . . . . . . . . . . . . . . 104

6.5 Saturation throughput comparison for network of 5-40 competing active nodes and with different percentage of hidden terminals, operating in RTS/CTS mechanism. . . . . . . . . . . . . . . . 105

6.6 Average delay comparison for a network of 5-40 active nodes, operating in RTS/CTS and basic access method (DCF \& game-theoretic design). . . . 105

6.7 Average collision rate comparison for a network of 5-40 active nodes operating in basic access (DCF \& game-theoretic design) method with 0-60 percent hidden terminal for each scenario. . . . . . . . . . . . . 112 
6.8 Average collision rate comparison for a network of 5-40 active nodes operating in RTS/CTS access (DCF \& game-theoretic design) method with 0-60 percent hidden terminal for each scenario. . . . . . . . . . . . . . 113

6.9 Normalised delay distribution comparison for a network of 5-20 active nodes, operating in basic access method (DCF \& game-theoretic design). The bin-size is 0.2 seconds. . . . . . . . . . . . . . . . . 114

6.10 Normalised delay distribution comparison for a network of 30-40 active nodes, operating in basic access method (DCF \& game-theoretic design). The bin-size is 0.2 seconds. . . . . . . . . . . . . . . . . 115

6.11 Normalised delay distribution comparison for a network of 5 -20 active nodes, operating in RTS/CTS access method (DCF \& game-theoretic design). The bin-size is 0.2 seconds. . . . . . . . . . . . 116

6.12 Normalised delay distribution comparison for a network of 30-40 active nodes, operating in RTS/CTS access method (DCF \& game-theoretic design). The bin-size is 0.2 seconds. . . . . . . . . . . . . 117

6.13 Average delay comparison for a network of 5-40 active nodes operating in basic access (DCF \& game-theoretic design) method with 0-60 percent hidden terminal for each scenario . . . . . . . . . . . . . . 118

6.14 Average delay comparison for a network of 5-40 active nodes operating in RTS/CTS access (DCF \& game-theoretic design) method with 0-60 percent hidden terminal for each scenario . . . . . . . . . . . . . . 118

6.15 Fairness comparison for a network of 5-40 active nodes operating in basic access method. . . . . . . . . . . . . . . . . . . 119

6.16 Fairness comparison for a network of 5-40 active nodes operating in RT-

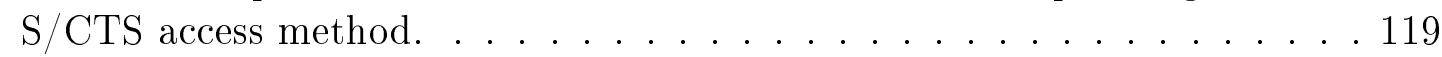

6.17 Fairness comparison for a network of 5-40 active nodes operating in basic access method with $0-60$ percent hidden terminal for each scenario. . . . . 120

6.18 Fairness comparison for a network of 5 -40 active nodes operating in basic

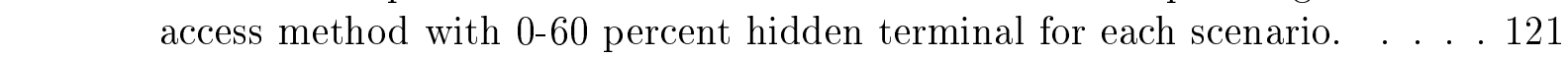

B.1 Normalised delay distribution comparison for a network of 5 active nodes, operating in basic access method (DCF \& game-theoretic design) with 1030 percent hidden terminal for each scenario. The bin-size is 0.2 seconds. . 138

B.2 Normalised delay distribution comparison for a network of 5 active nodes, operating in basic access method (DCF \& game-theoretic design) with 4060 percent hidden terminal for each scenario. The bin-size is 0.2 seconds. . 139

B.3 Normalised delay distribution comparison for a network of 5 active nodes, operating in RTS/CTS access method (DCF \& game-theoretic design) with 10-30 percent hidden terminal for each scenario. The bin-size is 0.2 seconds. 140

B.4 Normalised delay distribution comparison for a network of 5 active nodes, operating in RTS/CTS access method (DCF \& game-theoretic design) with 40-60 percent hidden terminal for each scenario. The bin-size is 0.2 seconds. 141 
B.5 Normalised delay distribution comparison for a network of 10 active nodes, operating in basic access method (DCF \& game-theoretic design) with 1030 percent hidden terminal for each scenario. The bin-size is 0.2 seconds. . 142

B.6 Normalised delay distribution comparison for a network of 10 active nodes, operating in basic access method (DCF \& game-theoretic design) with 4060 percent hidden terminal for each scenario. The bin-size is 0.2 seconds. . 143

B.7 Normalised delay distribution comparison for a network of 10 active nodes, operating in RTS/CTS access method (DCF \& game-theoretic design) with 10-30 percent hidden terminal for each scenario. The bin-size is 0.2 seconds. 144

B.8 Normalised delay distribution comparison for a network of 10 active nodes, operating in RTS/CTS access method (DCF \& game-theoretic design) with 40-60 percent hidden terminal for each scenario. The bin-size is 0.2 seconds. 145

B.9 Normalised delay distribution comparison for a network of 20 active nodes, operating in basic access method (DCF \& game-theoretic design) with 1030 percent hidden terminal for each scenario. The bin-size is 0.2 seconds. 146

B.10 Normalised delay distribution comparison for a network of 20 active nodes, operating in basic access method (DCF \& game-theoretic design) with 4060 percent hidden terminal for each scenario. The bin-size is 0.2 seconds. . 147

B.11 Normalised delay distribution comparison for a network of 10 active nodes, operating in RTS/CTS access method (DCF \& game-theoretic design) with 10-30 percent hidden terminal for each scenario. The bin-size is 0.2 seconds. 148

B.12 Normalised delay distribution comparison for a network of 20 active nodes, operating in RTS/CTS access method (DCF \& game-theoretic design) with 40-60 percent hidden terminal for each scenario. The bin-size is 0.2 seconds. 149

B.13 Normalised delay distribution comparison for a network of 30 active nodes, operating in basic access method (DCF \& game-theoretic design) with 1030 percent hidden terminal for each scenario. The bin-size is 0.2 seconds. .150

B.14 Normalised delay distribution comparison for a network of 30 active nodes, operating in basic access method (DCF \& game-theoretic design) with 4060 percent hidden terminal for each scenario. The bin-size is 0.2 seconds. . 151

B.15 Normalised delay distribution comparison for a network of 30 active nodes, operating in RTS/CTS access method (DCF \& game-theoretic design) with 10-30 percent hidden terminal for each scenario. The bin-size is 0.2 seconds. 152

B.16 Normalised delay distribution comparison for a network of 30 active nodes, operating in RTS/CTS access method (DCF \& game-theoretic design) with 40-60 percent hidden terminal for each scenario. The bin-size is 0.2 seconds. 153

B.17 Normalised delay distribution comparison for a network of 20 active nodes, operating in basic access method (DCF \& game-theoretic design) with 1030 percent hidden terminal for each scenario. The bin-size is 0.2 seconds. . 154

B.18 Normalised delay distribution comparison for a network of 20 active nodes, operating in basic access method (DCF \& game-theoretic design) with 4060 percent hidden terminal for each scenario. The bin-size is 0.2 seconds. 155 
B.19 Normalised delay distribution comparison for a network of 40 active nodes, operating in RTS/CTS access method (DCF \& game-theoretic design) with 10-30 percent hidden terminal for each scenario. The bin-size is 0.2 seconds. 156

B.20 Normalised delay distribution comparison for a network of 40 active nodes, operating in RTS/CTS access method (DCF \& game-theoretic design) with 40-60 percent hidden terminal for each scenario. The bin-size is 0.2 seconds. 157 


\section{LIST OF TABLES}

2.1 Summary of game-theoretic ALOHA-based protocols . . . . . . . . . . 36

2.2 Summary of game-theoretic CSMA-based protocols . . . . . . . . . . . . 37

$3.1 \quad$ DSSS system parameters and additional parameters used to obtain $\bar{n}_{i}^{\text {opt }}$. . 56

3.2 Medium Access Control Design $\ldots \ldots \ldots \ldots \ldots \ldots$

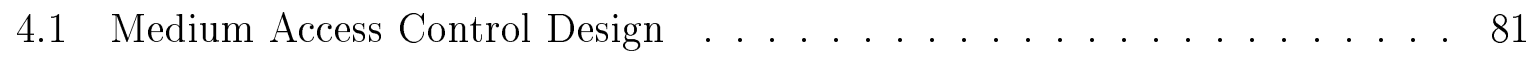

5.1 Generating random locations of nodes . . . . . . . . . . . . . . . . . 89

5.2 The number of hidden and covered stations in scenario depicted in Figure 5.4 from each node's point of view: . . . . . . . . . . . . . . . 91

6.1 Initial game parameters . . . . . . . . . . . . . . . . 101 


\section{List of ABBREVIATIONS}

ACK Acknowledgement

ADC Analogue to Digital Converter

AOB Asymptotically Optimal Backoff

AP Access Point

API Application Programming Interface

CCA Clear Channel Assessment

CDMA Code-Division Multiple Access

CSMA/CA Carrier Sense Multiple Access/Collision Avoidance

CW Contention Window

DCF Distributed Coordination Function

DIFS Distributed Inter-Frame Space

DSSS Direct Sequence Spread Spectrum

EDCF Extended Distributed Coordination Function

EIED Exponential Increase Exponential Decrease

EIFS Extended Inter-Frame Space

FDMA Frequency-Division Multiple Access

FSFCR Fairly Scheduled Fast Collision Resolution

IEEE Institute of Electrical and Electronic Engineers

LAN Local Area Network

MAC Medium Access Control

MACA Multiple Access with Collision Avoidance 
MACAW Multiple Access with Collision Avoidance for Wireless

MILD Multiplicative Increase, Linear Decrease

NAV Network Allocation Vector

NIC Network Interface Card

QoS Quality of Service

RTS/CTS Request to Send/Clear to Send

SDMA Space-Division Multiple Access

SSMA Spread-Spectrum Multiple Access

SIFS Short Inter-Frame Space

SINR Signal-to-Interference-plus-Noise Ratio

SNR Signal-to-Noise Ratio

TDD Time-Division Duplex

TDMA Time-Division Multiple Access 


\title{
LIST OF SYMBOLS
}

\author{
$N \quad$ Total number of nodes \\ $P_{i}^{\tau} \quad$ Channel access probability \\ $\tau_{V} \quad$ Vulnerable period \\ $s_{i} \quad$ Strategy of player $i$ \\ $s_{i}^{*} \quad$ Nash equilibrium strategy of player $i$ \\ $\mathbf{s}_{-\mathbf{i}}^{*} \quad$ Nash equilibrium strategy vector of all players except player $i$. \\ $\mathbf{S}_{\mathbf{i}} \quad$ Set of strategies of player $i$ \\ $\mathbf{s}^{*} \quad$ Nash bargaining solution strategy vector of all players \\ $u_{i}^{d} \quad$ Threat point \\ $P_{i}^{c} \quad$ Conditional collision probability \\ $\mathbf{P}^{\tau} \quad$ Channel access probability vector of all nodes \\ $\mathbf{P}^{\mathbf{c}} \quad$ Conditional collision probability vector of all nodes \\ $m \quad$ Backoff stage \\ $\mathcal{F}_{i} \quad$ Contention resolution algorithm \\ $\mathcal{C}_{i} \quad$ Conditional collision probability updates mechanism \\ $U_{i}($.$) \quad Utility function$ \\ $u_{i}($.$) \quad Payoff function$ \\ $P_{i}^{\tau *} \quad$ Nash equilibrium strategy of player $i$ \\ $\mathbf{P}^{\tau *}{ }_{i} \quad$ Nash equilibrium strategy vector of all players except player $i$. \\ $\mathbf{P}^{\tau *} \quad$ Nash equilibrium strategy vector of all players \\ $T_{s} \quad$ Time slot duration $[\mathrm{s}]$
}


$T_{c} \quad$ Average collision duration [s]

PHY $Y_{h d r}$ Physical layer header size [bit]

$M A C_{h d r} \quad$ MAC layer header size [bit]

L $\quad$ Packet size [bit]

RTS RTS header size [bit]

DIFS DIFS duration [s]

$\widehat{\sigma} \quad$ Mean propagation delay $[\mathrm{s}]$

br $\quad$ Basic rate $[\mathrm{bit} / \mathrm{s}]$

$v \quad$ Channel bit rate [bit/s]

$\bar{n}_{i} \quad$ Number of idle slots between two transmission attempts

$\bar{n}_{i \infty}^{\mathrm{opt}} \quad$ Optimal number of idle slots between two transmission attempts

$P_{i}^{\tau_{h}} \quad$ Probability that a hidden station transmits during the vulnerable period

$n_{c} \quad$ Number of the covered nodes

$n_{h} \quad$ Number of the hidden nodes

$P_{t r} \quad$ Probability of transmission in a time slot

$P_{s} \quad$ Probability of a successful transmission in a time slot

$E($.$) \quad Step size function$

$P_{i}^{i d l e} \quad$ Channel idle probability

SIFS SIFS duration [s]

CTS CTS header size [bit]

$A K C \quad$ AKC header size [bit]

$\Psi_{i} \quad$ Optimal number of idle slots matrix (for player $i$ ) 


\section{CHAPTER 1}

\section{INTRODUCTION}

Wireless devices are increasingly pervasive in everyday life. Coupled with the flexibility and mobility of wireless systems, technological advances are the driving force behind the "Anyone, Anywhere, Anytime" paradigm of networking. With laptops connecting to WiFi hotspots and cellphones streaming videos through cellular base-stations, users are demanding higher speeds and higher availability from their wireless networks. Even with the significant advancements made in wireless network designs over the last few decades, wireless networks are plagued with problems, such as location-dependent nature of carriersensing operation (that ultimately results in hidden terminals and exposed terminals problems), burst errors, and time-varying channel. An example that many readers would identify with is seeing a good signal level on the WiFi connection of a laptop and still experiencing page load errors in a web browser. Such problems are much more noticeable in highly crowded wireless environments, such as enterprise buildings or conferences with hundreds of users simultaneously using the wireless medium for data transfer.

Most challenges in wireless networking originate from the shared, broadcast nature of the wireless medium. In the broadcast networks, a single transmission medium is shared by a community of nodes [1]. For this reason, these networks are called multiple access networks (Figure 1.1). A shared transmission medium implies that communication devices need to contend amongst themselves, requiring specific sharing mechanisms to use 


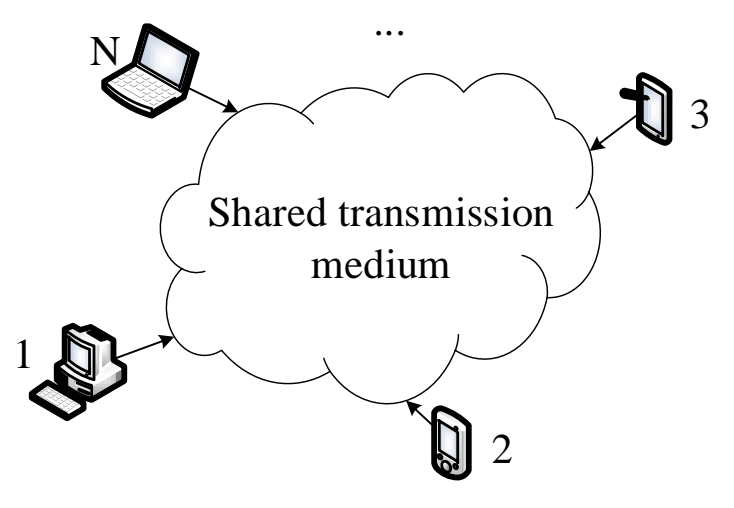

Figure 1.1: Shows a generic multiple access communication situation in which a number of stations share a transmission medium ( $N$ denotes the number of stations in this thesis).

the medium efficiently. These mechanisms consist of a set of rules, which are collectively called the medium access control (MAC) protocol.

The scope of this study must be defined before a whole network of wireless nodes ${ }^{1}$ trying to establish the proposed protocols, are considered. There are two broad categories of schemes for sharing a transmission medium [2]. The first category involves a static, collision-free, and contention-free channel access sharing of the medium, which is called a channelisation scheme because it involves the partitioning of the medium into separate channels that are then dedicated to particular users [1, 3]. Channelisation techniques are Time-Division Multiple Access (TDMA), Frequency-Division Multiple Access (FDMA), Code-Division Multiple Access (CDMA) or Spread-Spectrum Multiple Access (SSMA), Space-Division Multiple Access (SDMA), and channelisation techniques that are used in telephone cellular networks and lie outside the scope of this thesis.

The second category involves a dynamic sharing of the medium on a per frame basis that

\footnotetext{
${ }^{1}$ Wireless devices are called stations, terminals, or nodes in this thesis (generally in wireless network terminology, a station, terminal, and node are often used interchangeably with no strict distinction existing between these terms).
} 


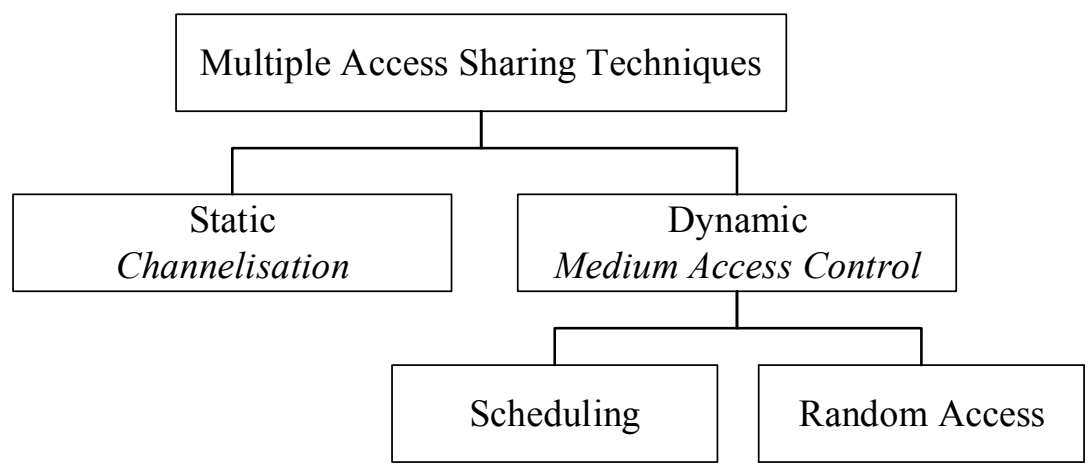

Figure 1.2: Approaches to sharing a transmission medium.

is better matched to situations in which the user traffic is bursty [1, 3]. This category is called medium access control schemes. The primary function of MAC is to minimise or eliminate collisions to achieve a reasonable utilisation of the transmission medium both in terms of channel access delay and channel throughput. MAC schemes can be classified based on the mode of operation into random and guaranteed access protocols ${ }^{2}$. As the title of the thesis suggests, MAC schemes and specifically random access protocols are the main focus. Figure 1.2 summarises the various methods to sharing a transmission medium.

In wireless networks, there are two types of architecture, centralised and distributed: Centralised architecture in wireless networks are extensions to wired networks with wireless in the last section of the network. This type of the architecture in the wireless networks are also known as last-hop networks. These networks have an access point (AP) that allows wireless devices to connect to a wired network. In centralized architecture, the down-link transmissions (from AP to stations) and the up-link (from stations to the AP) are shared by all the stations, therefore, this is a multiple access channel. The existence

\footnotetext{
${ }^{2}$ There is another classification of MAC which is hybrid access protocols. This type of MAC protocol uses random access protocols and scheduling protocols in hybrid fashion. Hybrid access protocols can only operate in a centralised architecture.
} 


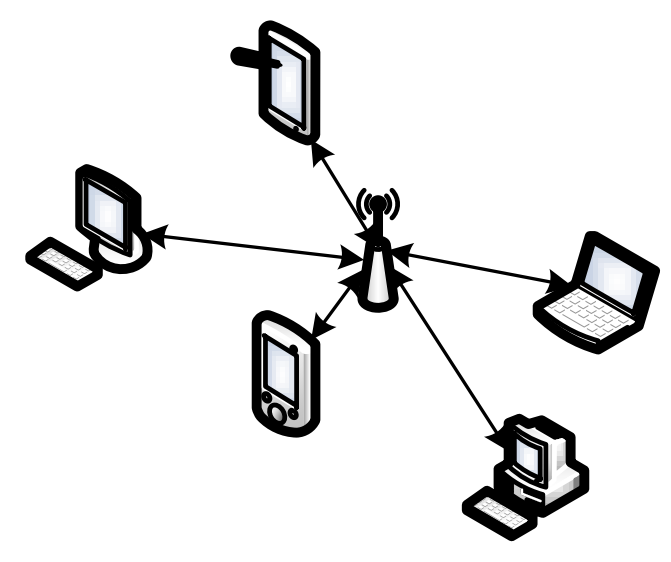

Figure 1.3: Typical centralised wireless network, also known as last-hop network.

of a central coordinator, such as an AP in this type of network architecture, gives a great degree of flexibility in using different MAC protocols [1, 3]. This network architecture can operate in all MAC schemes, including random access protocols, guaranteed access protocols, and hybrid access protocols. The AP can control the up-link transmissions by coordinating the channel access according to Quality of Service (QoS) requirements. The centralised wireless network architecture is shown in Figure 1.3.

Distributed wireless networks are wireless stations that communicate with one another in a infrastructure-less fashion. This type of architecture is also known as ad hoc networks. A typical ad hoc network is illustrated in Figure 1.4. In ad hoc networks wireless terminals exchange information between one another in a distributed manner. In this particular network architecture, all data transmission and reception must be in the same frequency band since there are no special nodes to translate the transmission from one frequency band to another. Therefore, all ad hoc networks can only operate using random access protocols. 


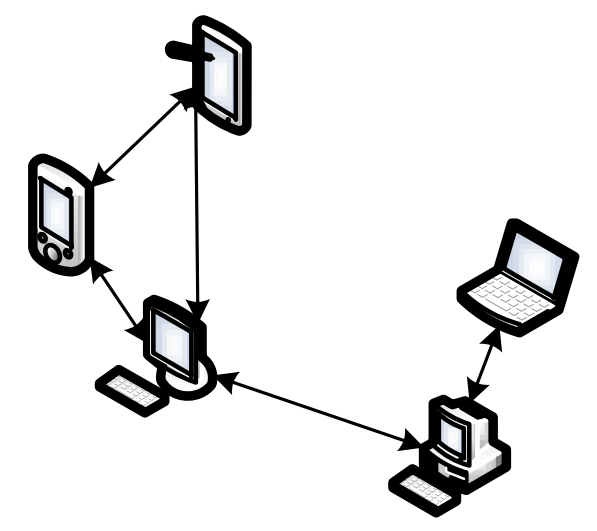

Figure 1.4: Typical distributed wireless network, also known as ad-hoc network.

\subsection{Wireless Random Access MAC Protocols Today}

Due to the simple nature of random access protocols, their operating mechanisms are generally straightforward. However, random access protocols are problematic in that a transmission is not guaranteed to be a success [4]. In random access protocols, a transmission is initiated without a prior negotiation with other possible transmissions, that ultimately can result in collision. This enables the protocol to accommodate large numbers of stations in the network without any increase in the computational complexity of its operation in order to allocate channels to individual stations. In random access protocols, when network traffic load is light, the probability of collisions becomes small, so retransmissions need to be carried out infrequently. Consequently, under light network traffic load, the channel access delay is low. On the contrary, when network traffic load is high, the probability of collisions increases, resulting in high channel access delay and low system throughput. For this reason, random access protocols require stations to use a backoff algorithm, which typically delays retransmission for the duration of a random amount of time. 
The history of random access MAC is now briefly discussed for the sake of explaining today's state-of-art MAC protocol for wireless local area networks (LANs).

\section{- $A L O H A$ :}

Norman Abramson and his team at the University of Hawaii Developed ALOHA random access protocol [5]. The system operation is simple. A radio transmitter is attached to the stations, and packets are transmitted as soon as they are generated, thus producing the smallest possible channel access delay. When two or more nodes transmit simultaneously, a collision will occur. This results in a corrupted packet. These collisions can be treated as transmission errors, and recovery can take place by retransmission? The retransmission mechanism uses a simple backoff algorithm without carrier sensing $]^{4}$ in order to minimise the probability of further collisions. In 1972, Robert et al. [6] proposed a method for doubling the ALOHA system performance. The proposal was to divide time into discrete intervals, each interval corresponding to one frame. This approach requires the nodes to send their packets during synchronised time slots. In order to achieve synchronization, Robert suggested [6] having one special node in the network to broadcast the start of each interval, like a clock. This method is called slotted ALOHA.

- Carrier Sense Multiple Access Protocols: Protocols in which nodes sense the carrier on the transmission medium and act accordingly are called carrier sense protocols. In 1975, Kleinrock et al. [8] proposed and analysed several protocols from this category, such as 1-persistent, nonpersistent, and p-persistent protocols.

When a node wishes to transmit, it senses the carrier on the channel. If it finds the

\footnotetext{
3"It is assumed that a protocol providing a reliable communication service that resides in the upper layers of the protocol stack and it will discover a failure of the transmission some time after the collision and will then trigger a retransmission" [5].

${ }^{4}$ By sensing the medium for the presence of carrier signal from other stations, a station can determine whether there is an ongoing transmission. When nodes sense the carrier on the transmission medium, none of them will attempt to use the medium until it becomes idle.
} 
channel to be busy, the node waits until it becomes idle. Otherwise, it transmits a frame. If a collision occurs, the station waits a random amount of time and starts all over again. In practice, the performance of the CSMA protocol is much better than ALOHA system. The reason is that nodes have the ability to desist from interfering with the other node's transmission. However, each variant of CSMA has its own problems. 1-persistent CSMA has poor fairness because a station with packets to send becomes too greedy and never loses hold of the channel. To overcome this problem nonpresistent CSMA was proposed, that was made to be less greedy than in the 1-persistent variant. Before sending, a station senses the carrier on the channel. If the channel is idle, the station initiate its transmission. However, if the channel is already in use, the station does not continually sense it for the purpose of seizing it immediately. Instead, it waits a random period of time and starts all over again. This protocols has better channel throughput but longer channel access delays than 1-persistent CSMA. To deal with delay problem and providing better channel utilisation, the p-persistent protocol was proposed. In this protocol stations transmit with a probability $a$ and they defer transmission attempts with a probability $b=1-a$ until the next slot. Nevertheless, all CSMA protocols are not practical for today's wireless networking demands. The lack of ability to deal with problems such as the hidden and exposed terminal (cf. \$2.1.2) shows they can not operate reliably with large numbers of competing node. Moreover CSMA protocols do not have appropriate collision avoidance mechanisms $5^{5}$ to operate in wireless medium.

\section{- Multiple Access with Collision Avoidance for Wireless (MACAW):}

One of the first protocols designed specifically for wireless networks was MACA (Multiple Access with Collision Avoidance) [7]. The basic concept behind its oper-

\footnotetext{
${ }^{5}$ These mechanisms are explained in context of distributed coordination function (DCF) in $\$ 3.2 .1$.
} 
ation is that the node that intends to transmit uses the short frame called request to send (RTS) in order to stimulate the destination node to respond using another short frame called clear to send (CTS). Thus, the stations nearby receive CTS frame and detect this transmission. Consequently, they avoid transmitting for the duration of the upcoming (large) data frame. Later on, Bharghavan et al. [8] suggested including an ACK frame to MACA after each successful data transmission in order to make this protocol more reliable and also to improve its performance. They called the new protocol MACA for Wireless (MACAW). This protocol became the foundation for today's standard 802.11 distributed coordination function (DCF). In this study, the DCF was used as the reference protocol. The detailed description of DCF is given in Chapters 3 and 4.

IEEE 802.11 DCF is the de facto MAC protocol for WLANs because it is one of the most stable distributed protocols that has been ever built. However, it has a serious shortcoming: The choice of contention resolution algorithm and contention measure is the key to the performance of medium access control protocols, and the inappropriate choice of contention measure and contention resolution will and does result in poor performance [9, 10. DCF uses the binary exponential backoff mechanism as one of its collision avoidance methods, where each node doubles its contention window (CW) after each collision. This collision avoidance mechanism results in too many collisions and, subsequently, leads to poor channel utilisation as the size of the network increases [10]. Moreover, DCF also has short-term fairness problem due to the exponential backoff mechanism applied after each collision, which results in unwanted oscillation in the size of contention window simply because DCF cannot distinguish collisions from corrupted frames. Designing efficient MAC protocols provides motivation for addressing these issues in the DCF. This leads to the fundamental questions on how to design efficient MAC protocols and how to determine which methodology and guidelines to follow. Designing efficient MAC protocols is a 
challenging task, especially in wireless environments where channel sensing is much less reliable than in a wired medium. Therefore, an efficient MAC protocol should satisfy the following properties:

- An efficient MAC protocol, at some point of operation, must converge to a stable equilibrium. The converged stable equilibrium must be optimal for the whole network, which means each node must get a fair share of payoff and good channel utilisation at the equilibrium operating point.

- An efficient MAC protocol must be able to survive in a non-compliant environment: In autonomous wireless networks, nodes may act selfishly and do not cooperate as an entity in a wireless network. These nodes may adapt the strategy that maximises their own utility. Therefore, under such circumstances, an efficient MAC protocol must be able to survive in a way that each node can reach the stable equilibrium even if the rest of the network operates in a self-interested and noncooperative fashion.

\subsection{Game Theory for Wireless MAC}

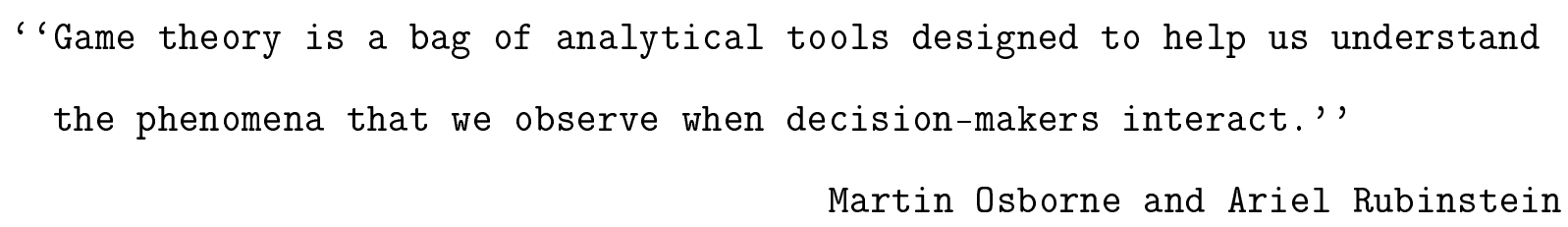

The study is conducted to design an efficient MAC protocol using game theory. In this section, the motivation behind adopting a game theoretic approach rather than global optimization approach is discussed. The discussion starts by asking, why is game theory the most promising candidate for conducting this study?

Game theory is a branch of mathematics concerned with the analysis of interactive decision-making processes. It provides mathematical basis for predicting what might 
(and possibly what should) happen when rational agents with conflicting interests interact. It is not a single monolithic technique, but a collection of modelling tools that aids in the understanding of interactions amongst the decision-making processes. A game has three basic components: a set of players which are the decision makers in the modelled scenario, a set of actions, and a set of preferences. For a MAC protocol to be designed efficiently, the players are most often the nodes of the network. The actions are the alternatives available to each player. In extensive or dynamic form games, the set of actions might change over time. In a wireless MAC protocol, actions may include the transmit power level, channel access probability, or any other factor that is under the control of the node [11, 12]. The outcome of the game is determined by the result "action profile" when each player chooses an action. Finally, a preference relationship or in many cases, utility function evaluates all possible outcomes from each node's point of view. An action with higher utilities representing more desirable outcomes. In the wireless MAC scenario, outcomes that yield higher channel utilisation, lower channel access delay, and better fairness might be preferred by a player, although in many practical situations, these goals will be in conflict [11]. Appropriately modelling these preference relationships is one of the most challenging aspects of the application of game theory to MAC protocol design.

Wireless networks using DCF protocols have occupied a preeminent place in the wireless networking literature for the last several years (cf. 2.2.3). A DCF is a self-configuring protocol in which no central coordinator is presented, even in the centralised configuration. Thus, every aspect of the configuration and operation of a DCF protocol is completely distributed. In a modern wireless network, each node running a distributed protocol must make its own decisions (possibly relying on information from other nodes). These decisions may be constrained by the rules or algorithms of a protocol, but ultimately, each node will have some leeway in setting parameters or changing its mode of operation [11]. These nodes, then, are autonomous agents, making decisions about transmit power, back- 
off time as the choice of channel access probability, and so on. In making these decisions, what does the node seek to optimize?

In some cases, nodes may seek the "greater good" of the network as a whole. In other cases, nodes may behave selfishly, looking out for only their own user's interests [11]. In a final case, nodes may behave maliciously, seeking to ruin network performance for other users [11, 12]. In the second and third cases, the application of game theory is straightforward as game theory traditionally analyses situations in which player objectives are in conflict

[11. In the first case, node objectives are aligned (as all players seek the "greater good" of the network), but game theory may still offer useful insights [11, 12, 13]. Even when nodes have shared objectives, they will each have a unique perspective on the current network state (e.g., networks with presence of hidden terminals), leading to possible conflicts regarding the best course of action.

\subsection{Contributions}

This thesis proposes and explores the game-theoretic approach as a new paradigm for designing distributed wireless MAC protocols. It adopts a noncooperative, static, gametheoretic approach to model DCF in autonomous wireless networks where each node chooses its strategy, which is the channel access probability, to maximise its utility function. In noncooperative games, the players make rational, self-enforcing decisions considering only their individual payoffs. Due to the nature of the wireless channel, static games are employed, which means all players make decisions without the knowledge of the remaining competing players' strategies. The idle sense method is employed to design the utility and payoff functions, as proposed by Heusse et al. [14]. This derives the optimal number of consecutive idle time slots between transmissions, dependent on the channel modulation scheme, in order to avoid or minimise contention. This means at the Nash equilibrium (cf. 3.2 .3 for definition of Nash equilibrium in the game) the proposed non- 
cooperative games produce the strategy profile where no players (nodes) have incentive to deviate unilaterally. Thus, the proposed game-theoretic MAC protocols operate as the distributed strategy update mechanism, approaching the Nash equilibrium by locally observing the consecutive idle slots between transmissions. To this end, two games are proposed. The first game is designed for single-cell ad hoc networks [15]; the other game is designed for centralised networks using DCF in the presence of hidden terminals [16]. Specifically, the proposed MAC protocols make the following contributions.

- Game-theoretic MAC protocols decouples the channel access probability from the conditional collision probability by estimating the conditional collision probability through observation of consecutive idle slots on the medium in order to provide the ability to discriminate between channel contention and occasional channel lack of reliability and to minimise the effect of de-synchronisation amongst covered and hidden nodes.

- The proposed game-theoretic random access models provide an appropriate contention window size independently for each node in the network. This yields an appropriate channel access probability for all nodes in the presence of hidden terminals and provides an almost equal channel access probability for all nodes in single-cell ad hoc network only through local observation of consecutive idle slots between transmissions.

These contributions results in significant improvement to channel utilisation and fairness and ultimately lower channel access delay in all circumstances, such as in the presence of hidden terminals, high error rates on the channel and etc, where compare to DCF as base line protocol. 


\subsection{Outline}

The thesis is organised as follows. Chapter 2 provides a background to distributed medium access protocols, identifying the current state-of-the-art protocols, and explores the designs that improve distributed protocols using game-theoretic and global optimization approaches. This chapter explains challenge in game theoretic designs of multiple access protocols and provides comprehensive details on previous work in this area. Chapter 3 proposes and describes the design of a game-theoretic medium access method for singlecell ad hoc networks. This chapter explains how the proposed MAC protocol can be integrated into DCF and exhibits stable protocol behaviour. Chapter 4 describes the design of a game-theoretic access method for a centralised network operating in a distributed manner. This chapter addresses the issue of hidden terminals by designing utility function using an appropriate Markov model that takes the effect of hidden terminals into account. Chapter 5 provides the simulation methodology employed, including the validation of the chosen network simulator, Omnet ++ , and compares it against a number of other network simulators. It also introduces and discusses the performance metrics, network topology, simulations parameters, and simulation scenarios used in this study and upon which the discussion of further chapters has been based. In Chapter 6, the simulation results of the proposed protocols are analysed and compared with DCF as baseline protocol, focusing on their throughput, collision rate, fairness, and average channel access delay. Finally, in Chapter 7, the thesis is concluded with a discussion of the main findings and possible avenues for future work. 


\title{
CHAPTER 2
}

\section{CONTENTION-BASED RANDOM CHANNEL}

\section{ACCESS SCHEMES}

\author{
"It is generally not possible for radios to receive and transmit \\ on the same frequency band because of the interference that res- \\ ults. Thus, bidirectional systems must separate the uplink and \\ downlink channels into orthogonal signalling dimensions, typica- \\ lly using time or frequency dimensions.',
}

- Andrea Goldsmith, 'Wireless Communications,', Cambridge University Press.

\subsection{Wireless Medium from Random Channel Access Point of View}

Random access protocols are a well-researched topic in both wired and wireless systems. Due to the nature of wireless networks medium, the design procedure for wireless network MAC protocols notably different to those of wire line networks, and is also considered much more challenging. In order to design an efficient MAC protocol, there are various issues relevant to the wireless medium that need to be considered. 


\subsubsection{Single channel Half-duplex}

One of the key principles associated with wireless communication is the fact that a radio is unable to both receive and transmit simultaneously at the same frequency. In other words, a radio cannot operate in a full-duplex fashion in wireless networks. This is because of the fact that wireless signals attenuate quickly over distance. Thus, the signal power from a local transmitting antenna is significantly stronger than those from other nodes. Accordingly, collision detection is not an option for single channel half-duplex wireless networks. To deal with this issue, wireless nodes that are running random access protocols must separate the uplink and downlink using Time-Division Duplex (TDD), also known as half-duplexing.

In the wireless network, TDD can be viewed simply in terms of time slots; when data is to be sent between nodes, access in time is shared, which breaks up time into respective time slots. Subsequently, the nodes take turns to send data to one another. Channel time division, applied in such a way, prevents interference in transmissions when two nodes are transmitting. Various different networks utilise TDD owing to the fact that its application is relatively straightforward-particularly in the case of ad hoc networks in which frequency use is neither strictly coordinated nor controlled (e.g., wireless LANs (802.11), Bluetooth and Zigbee [17]). Nevertheless, the utilisation of time-division duplexing aggravates any channel-level inconsistency across nodes. The fact that only one node in every communicating pair is able to transmit data at any particular time, the transmitting node's surrounding wireless channel may appear to be engaged, whilst the receiving node's surrounding wireless channel may appear vacant. These types of discrepancy are one of the key reasons behind the numerous issues and challenges apparent with TDD wireless networks, including hidden terminal effects and packet losses as a result of collisions, etc. 
There are a number of suggested approaches ${ }^{1}[18,19$, 20, 21, 22, 23, 24] for the design of a single-channel, full-duplex wireless radio, utilising interference cancellation for the eradication of the self-interference signal from the receiver of the wireless node. Theoretically, such an obstacle should be simple to overcome: for a system with separate antennas for receiving and transmitting, owing to the fact that the system knows the signal of the transmit antenna, it is able to subtract it from the signal of the receiving antenna and then decode the remainder. However, in practice, overcoming this obstacle is much more problematic: the receiver circuitry — particularly the ADC (analogue to digital converter) - is saturated through strong self-interference, which makes it impossible for a packet to be decoded by the receiver following the deduction of the self-interference signal. Thus, this impedes the application of a full-duplex system utilising only digital cancellation [22]; nonetheless, such a cancellation approach cannot function when there is a moderately high-power, wideband wireless signal, as in the case of the 802.11 (WiFi) because of ADC saturation.

To conclude, because of the fact that full-duplexing is not considered practical for distributed networks, and also because TDD operation would not facilitate the detection of the collision by nodes, as in the case of the wired networks, all suggested MAC protocols for TDD systems need to implement collision-avoidance approaches $2^{2}$ One of the key collision-avoidance instruments in the majority of all distributed MAC protocols is carriersensing; this is commonly adopted with the aim of lessening the likelihood of an in-network collision (decreasing the probability of a collision). Nonetheless, this tool can induce a number of other challenges, as discussed in the following part.

\footnotetext{
${ }^{1}$ Wireless researchers have used interference cancellation techniques to either exploit collisions [18] or recover from collision losses [19, 20]. Full-duplexing using some cancellation techniques has also been explored in the literature. Analogue cancellation techniques using noise cancelling chips have been proposed to subtract the self-interference signal (the "noise") from the received signal [21]. Digital cancellation, used in CSMA/CN [22, optical networks [23], and proposals for full-duplex operation [24], subtracts selfinterference in the digital domain, after a receiver has converted the baseband signal to digital samples.

${ }^{2}$ Collision avoidance principles belonging to DCF are discussed in detail in Chapter 3 and 4.
} 


\subsubsection{Location-dependent carrier sensing}

Over distance, wireless signals are known to attenuate according to a power law distance dependency; this is usually attributed to multipath propagation. Accordingly, in line with the transmitter position, the position of the receiver implements carrier-sensing, meaning that, in the network, wireless nodes only have the ability to sense the presence of one another within a restricted range; this is dependant on the sensitivity of the receiver as well as the power of the transmitter. Accordingly, such location dependency will result in various problems, some of which stated below:

- Hidden Terminals: This issue arises when a node is visible from a set of specific nodes in the network, but not from other nodes communicating with that set of specific nodes.

- Exposed Terminals: This problem goes hand-in-hand with the hidden terminal issue. This arises when a node is stopped from transmitting to other nodes as a result of the occupancy of the wireless medium channel by a nearby carrier signal, meaning the message recipients intended by the node are not reached.

- Capture: This arises when a receiver is able to easily and efficiently receive a transmission from one of two simultaneous transmissions-both within its range. The overall efficiency of the network in a distributed structure might be increased; however, in the case of a centralised architecture, those nodes that are closely aligned with AP could seize the channel more often, thus resulting in fairness issues amongst nodes in the network.

Game-theoretic designs to address these problems in 802.11 are discussed in Chapters 3 and 4 


\subsubsection{Time-varying channel}

Multipath propagation in a wireless medium causes the received signal at each node, to become a superposition of attenuated and time-shifted versions of the transmitted signal. Moreover, the received signal varies as a function of time due to the movement of scatters. Therefore, in a time-varying channel, the received signal may not be recognised by the destination node. In this case, the destination node is said to be in a fade. In order to mitigate this problem, which is a direct result of constant change of signal characteristics in a time-varying channel, handshaking is widely used: Nodes that wish to communicate with each other use small packets without payload to test the quality of the channel between themselves. However, the changing characteristic of channel over time makes nodes more vulnerable to receiving corrupted frames. Ultimately, this increases the error rate on the channel. This phenomenon is called Burst Channel Error [1]. To address this problem, the link-layer provides a reliable communication channel by acknowledging the transmitted signal or in the case of not receiving an acknowledgement from the destination, by triggering the retransmission procedure.

\subsection{The Design of MAC for Random Channel Access Schemes}

The key parameters that need to be considered in order to design and optimise a multiple channel access scheme are network throughput, network delay, and fairness. Network throughput is the volume of data transmitted successfully across the nodes within a particular timeframe. Network delay refers to the time spent by nodes in the network to successfully transmit their packets, whilst fairness considers whether or not network nodes utilise their fair share of channel capacity within a long or short period of time. Wireless system properties and their associated medium-notably time-varying channel, 
location-dependent carrier-sensing, and half-duplexing - cause inadequate fairness, long delays, low throughput and, low level of power efficiency, due to aforementioned reasons.

A number of experiments have been carried out in real-world environments with the aim of examining the wireless medium with the implementation of the wireless LAN protocol. Based on an 802.11 centralised testbed in the Divert project, experiments showed notable frame losses as a result of radio channels' time-varying behaviours - even when nodes were fairly close to the AP [25]. Moreover, it was also observed that frame losses were identified in bursts, with their rate significantly depend on the path between a node and an access point. In Roofnet [26], extensive measurements showed that frame loss rates distribution is comparatively uniform across the entire loss rates range. Furthermore, both distance and Signal-to-Noise Ratio (SNR) have very little predictive value for loss rate [26]. An additional research based on ad hoc networks showed that the transmission range in ad hoc networks is not circular, communications amongst nodes is not symmetric, and the wide variation in the average signal strength - even amongst those nodes whose positions are relatively close to an AP [27]. As another example [28], the authors measured bit-rate diversity amongst nodes in the context of centralised wireless LANs, showing that, when nodes are comparatively close to the AP, as in the case in an indoor environment, more than half of all data packets were transferred with the use of the lowest bit rate rather than the higher one. Other experiments [29] examined the physical layer capture effect in consideration of 802.11 wireless LANs, with the finding established that a stronger signal might be successfully received by a node if a data packet is transmitted with the use of a stronger signal involved in a collision. Such an occurrence induces significant inequality in terms of throughput amongst contending nodes; this induces notable inequitableness owing to the fact that the node that detects the collision doubles its contention window; this decreases the channel access probability. However, the successful node achieves a larger share of the channel in two different ways: It performs its frame transmission and 
continues to function with the use of the minimum contention window, which provides a rationale for why the 802.11 is recognised as having a short-time fairness issue, that also leads to a long-time fairness issue.

The performance analysis of a node's behaviour in wireless networks and particularly in 802.11 wireless LANs will be briefly reviewed below. The performance analysis of a node's behaviour will be used later for designing the utility and payoff function in the proposed game-theoretic designs. Subsequently, a number of the designs that propose enhanced contention resolution algorithms related to this study are discussed in order to identify the shortcomings of the 802.11 wireless MAC.

\subsubsection{Performance analysis of DCF in single-cell wireless net- works}

Kleinrock and Tobagi [30] proposed the first model to evaluate the 1-persistent, p-persistent, and non-persistent CSMA. Huang et al. [31] evaluated non-persistent CSMA with fourway handshaking methods in multi-cell wireless networks by taking hidden node problems and cell-overlapping into account. Ray et al. 32] proposed a detailed analysis method based on queuing theory to evaluate the effect of hidden terminals on the mean packet delay in CSMA protocol without considering the backoff time between consecutive transmissions. Chhaya [33] and Wang et al. [34] introduced the concept of the vulnerable period ${ }^{3}$ and developed a model for the collision probability in the network based on the length of the vulnerable period. However, these models [30, 31, 32, 33, 34] are not able to analyse a node's behaviour in IEEE 802.11 DCF because they ignore the binary exponential backoff procedure, which is one of the most important collision avoidance principles incorporated in DCF.

In 1997, the IEEE 802.11 protocol [17] was the first wireless networking protocol to be

\footnotetext{
${ }^{3}$ The concept of vulnerable period is discussed in context of IEEE 802.11 DCF for centralised wireless network architecture in $\$ 4.1 .2$
} 
standardised for wireless LANs. Since then, a large number of performance analysis studies have focused on modelling the DCF behaviour in wireless networks. The first coherent model for 802.11 DCF was proposed by Bianchi [9] He defined the conditional transmission probability for a node as the sum of all the probabilities corresponding to the node's backoff counter as it reaches zero during a randomly chosen slot time. He assumed that transmission probability is constant and independent of the number of retransmissions (which can be interpreted as the DCF backoff mechanism). Furthermore, he pointed out that this assumption is fairly accurate when the size of the contention window and the number of nodes is relatively large. Finally, based on the transmission probability, he derived the saturation throughput of DCF, which incorporates the binary exponential backoff mechanism. This model was the first model to elucidate the DCF backoff procedure in terms of channel access probability. However, this model did not consider the effect of hidden terminals and networks with nodes in the nonsaturation condition. After his proposed model for DCF, many modified models were proposed considering various aspects of 802.11: Wu et al. [35] used the Markov chain model like Bianchi's [9], which considers frame retry limits to arrive at a more accurate calculation of DCF throughput. Alternatively, Ziouva et al. [36] used the Bianchi model [9] by considering contention window time slots being recounted as a result of a frozen timer when the wireless medium is sensed busy by the carrier sensing procedure in DCF. In [37, 38], Bianchi's model [9] is extended to analyse the network throughput when nodes are in both nonsaturation and saturation conditions. An additional transition state was used to model the nonsaturation condition to present nodes that have no packets to transmit in the network.

\footnotetext{
${ }^{4}$ We use Bianchi's model for design of the utility function in Chapter 3 . For more details see $\$ 3.2 .2$
} 


\subsubsection{Performance analysis of DCF in the presence of hidden ter- minals}

The DCF models discussed above have been established based on the perspective that the DCF network is single-cell. In other words, the assumption is held that, within the network, there are no hidden terminals. Simulation [39] shows that Bianchi's model [9] is not valid for the analysis of DCF networks with the presence of hidden terminals. Several studies [40, 41, 42, 43, 44, 45] were proposed to model the effect of hidden terminals on the overall network performance. These studies can be divided into two groups: The first group [40, 46, 41] considers networks with small numbers of nodes and only two contending stations. The second group [42, 43, 44, 45] performs the analysis for large numbers of contending nodes in the network. The first group of models are fairly accurate; however, they cannot be used for realistic network scenarios because of lack of clarity in terms of how they may be extended to networks with large number of active nodes (e.g. $N \geqslant 10$ ). Kim et al. [40] modelled the coupling effect $t^{5}$ with the application of Bianchi's model for the network with small numbers of nodes $(N \leqslant 8)$. The work illustrated that the probability of collision significantly depends on the DCF backoff approach. The model was amended and modified so as to include the coupling effect of the conditional collision probability within the network where nodes are recognised as being in saturation. A special scenario was adopted where the hidden terminals and opposing nodes were categorised into two different areas, with the contending nodes found to be equal to the number of hidden terminals. In a similar vein, a phenomenon was introduced by Tsertou and Laurenson [46], centred on the lack of time-synchronisation amongst hidden terminals. The research emphasised that hidden terminals are not able to decrement their backoff counters upon the occurrence of a collision within the network, and that the synchronisation of backoff

\footnotetext{
${ }^{5}$ The coupling effect is the effect between hidden and covered node in process of seizing the channel
} 
timers will only be witnessed following successful transmission; thus suggests that the duration of the vulnerable period ${ }^{6}$ is double that previously believed to be the case. In consideration to this conclusion, the authors also maintained that renewal theory - such as Poisson and Bianchi's Markov models - is not able to sufficiently represent the transmission probability because of the lack of time-synchronisation amongst stations. Although, in [46], an accurate model was suggested with the aim of presenting the impacts of hidden terminals but, their model is valid only for networks with only two nodes contending at same time. They also used a fixed-size backoff counter as opposed to a binary exponential backoff mechanism in DCF. Furthermore, Kim and Choi [4] proposed presenting the conditional collision probability in saturation mode based on an ad hoc network with two sets of contending station pairs.

A model was presented by Ekici and Yongacoglu [42, which was concerned with the calculation of the collision probability between contending nodes and hidden terminals in a centralised WLAN. Similarly, Tsertou et al. [43] presented a model based on conditional collision probability between two contending pairs in the context of an ad hoc network. The postulation was made that the probability of a hidden terminal gaining access to the channel in each backoff slot is independent of the probability of hidden nodes gaining access to the channel in any of the previous slots. Nonetheless, such a postulation $]^{7}$ goes against the fundamental characteristics of the Markov chain; this necessitates that the transmission probability in each backoff slot rests only on the state of the node for the duration of the previous time slot alone. In order to overcome this issue, 44, 45] considered the probability of hidden terminal transmission during the vulnerable period as another parameter in their node's behaviour analysis. However, their model can only be used in a network with hidden terminals present.

\footnotetext{
${ }^{6}$ c.f. $\$ 4.1 .2$

${ }^{7}$ If $P_{i}^{\tau}$ denote the channel access probability in a randomly chosen slot time, then based on [42, 43] the probability that a hidden terminal will not transmit during the vulnerable period $\tau_{V}$ is $\left(1-P_{i}^{\tau}\right)^{\tau_{V}}$.
} 
The performance analysis model in [39] was used for designing the utility function in chapter [4]. [39] devise an analytical framework geared towards the assessment of the performance of the centralised 802.11 DCF in both nonsaturation and saturation conditions; this was achieved with the utilisation of a spatial-temporal analysis and a two-dimensional Markov chain. Importantly, the framework can be applied not only in cases where hidden terminals are present, but also in the case of networks without hidden terminals. Moreover, their model is simpler when compared with similar models, such as [44].

\subsubsection{Enhancing contention resolution algorithms}

There have been a number of proposals made in regard to the numerous improvements to be implemented for 802.11. Protocol designs proposing better contention resolution algorithms to improve throughput, mostly by tuning the contention window in order to be comparable with the proposals, are now briefly discussed.

The optimal value of the contention window (CW) was calculated by Cali et al. [47. Comparably, Gannoune et al. [48] suggested an approach for the calculation of the minimum CW size, whilst Bianchi et al. 49] presented an approach for predicting the amount of active hosts through the use of a Kalman filter to establish appropriate CW values. In an attempt to overcome the complexity associated with such an approach, a centralised strategy, involving the AP measuring the number of active nodes in the network then broadcasts the optimal CW size across all contending nodes, was proposed by Ma et al. [10]. Furthermore, a very complicated and decentralised approach was suggested by Bianchi et al. [50] and Cali et al. [47] with the aim of predicting the number of contending nodes in the network and accordingly calculating an optimal CW for the maximisation of network throughput. However, their estimation is fragile because it is highly dependent on the estimation of the conditional collision probability. A new backoff algorithm was presented by Bononi et al. [51] with the aim of improving an 802.11 DCF; this is known

\footnotetext{
${ }^{8}$ For more details see $\$ 4.1 .3$
} 
as Asymptotically Optimal Backoff (AOB), and has performance objectives comparable with [14].

Aad et al. 52 proposed a method to enhance the DCF efficiency and fairness by dividing the CW by two instead of resetting it to its initial value of $C W_{\min }$, treating it like the binary exponential backoff mechanism after a successful transmission (cf. \$3.2.1 for the formal binary exponential backoff detail) in order to provide a less disproportionate CW for nodes in the network. This method is called slow CW decrease. Conversely, a fast collision resolution algorithm was proposed by Kwon et al. [53], based on throughput improvement. The backoff mechanism acts like the DCF backoff when any node that either experiences a collision or loses a contention (it doubles the contention window size), but when the backoff timer begins the countdown stage, nodes can exponentially lower their backoff timer if they come to identify number of idle slots on the channel. In comparison to DCF backoff mechanism, the fast collision resolution algorithm significantly improves the throughput. However, it causes a reduction in the short time fairness because only the node that has just succeeded in accessing the channel can minimise its CW. To address this issue, Kwon et al. [53] have introduced the fairly scheduled fast collision resolution (FSFCR) method, which simply sets a limit on the amount of successive retransmissions which a node may perform: upon the reaching of a limit by a station, the $\mathrm{CW}$ is set to $C W_{\max }$.

In [54], an approach based on decreasing the collision overhead-referred to as the binary countdown strategy — was defined by the authors. Because of the fact that, collisions significantly deteriorate the throughput of the $802.11 \mathrm{DCF}$, the transmission of management messages was proposed with the aim of scheduling each transmission. Nonetheless, this management technique is recognised as taking up as much as one-fifth of the channel's overall capacity [54].

Some proposals have been made based on node cooperation to meet requirements, 
such as high throughput, lower delay, and better fairness. In an attempt to deal with the hidden terminal issue, Multiple Access Collision Avoidance - referred to as MACA- 7 was suggested, which utilises RTS/CTS as a collision-avoidance principle on the shared channel. In line with MACA, there is the proposition of a new protocol for wireless network LANs: the MACA for wireless (MACAW) was created with the aim of establishing fair channel allocation and high throughput with the utilisation of ACKs frames to accompany RTS/CTS ones [8]. The MACAW backoff mechanism suggested is based on the multiplicative increase, linear decrease (MILD) principle, whereby the backoff counter of each node is increased by a factor upon a collision or a corrupted frame, with the backoff timer decreased by one following transmission success. The approach applies the same backoff counter value for all nodes, as distributed in the packet header and copied by the receiver into its own counter.

Nandagopal et al. [55] introduced the proportionally fair contention resolution (PFCR) in which nodes control a transmission probability using the MILD principle to impose fairness among nodes when flows in the network face diverse spatial and contention conditions. However, imposing fairness at the level of flows must not be done at the MAC layer. It can be argued that flow control is the problem of traffic management, and it should be handled at an upper layer, such as the transport layer. Similarly, Song et al. [56] proposed a new backoff algorithm based on the EIED (exponential increase, exponential decrease) principle, in which each node increases its backoff counter by a factor upon a collision or corrupted frame and decreases the backoff timer by a different factor after a successful transmission. This method does well in comparison with MILD and the binary exponential backoff, which is used as standard backoff mechanism in 802.11 DCF.

The main problem with all these proposals is related to the core principle of dynamic load control. When a network node experiences a collision in all instances, the CW size of the node is increased in an attempt to decrease the probability of collision. This results 
in lower channel utilisation and fairness owing to the fact that the previously discussed proposals for enhancing DCF are unable to differentiate between collisions from corrupted frames; in addition, they do not have the ability to appropriately handle the capture effect [29]. In the case of the above methods, the CW size is usually increased significantly following a collision, and the increased size of the CW is almost never optimal. To address these issues, Heusse et al. [14] proposed a novel idle-sense access method for a single-cell wireless LAN, which compares the mean number of consecutive idle slots between transmission attempts to the optimal value and adopts an additive increase and multiplicative decrease algorithm to dynamically control the contention window in order to improve throughput and short-term fairness. As the idle-sense method relies on only observing idle periods in channel activity, this method is insensitive to all the problems that arise in the above methods based on inferring the channel load from collisions. In the proposed access methods (cf. Chapters 3 and 4), conditional collision probabilities are estimated by wireless nodes through observations of consecutive idle slots between transmissions similar to idle-sense. Accordingly, as in the case of the idle-sense, the proposed access methods can separate handling failed transmissions from contention control. Similar to idle-sense method, Hu et al. [57] proposed a channel access method to maximise the bandwidth utilisation and achieve proportional bandwidth allocation. This approach ultimately rests on to locally observable variables, namely the amount of collisions witnessed between two consecutive successful transmissions and the amount of consecutive idle slots, based on which to control the de-queueing rate of wireless nodes instead of dynamically tuning their contention windows.

A different approach is shown by Tan et al. [28], who proposed the placement of a regulator above the MAC layer in an $\mathrm{AP}$ with the aim of controlling the set and cell rates for nodes in line with various performance-related goals (commonly throughput or time fairness). Despite the fact that the concept may be seen to be valuable from the 
perspective of achieving equal time shares across stations, it remains that there is much dependence on a central coordinator and the access method does not address the hidden terminals problem.

\subsection{Game Theory and its Application to Random Chan- nel Access Schemes}

A game in random channel access schemes includes a set of players, actions, and preferences or player payoffs. Commonly, the players are the wireless nodes when MAC protocols are designed [11]. The actions are the alternatives available to each player. An action selected by a player, is referred to as the strategy. In the case of a wireless MAC protocol, actions might comprise the probability of channel access, the level of transmit power, CW size, as well as a number of other elements within the player's control. Upon the deterministic selection of the action, this is referred to as pure strategy. In contrast, upon the probabilistic selection of the action in line with a particular probability distribution, this is referred to as mixed strategy. Ultimately, the "action profile" established based on selection of an action by all players will determine the outcome of the game.

For all players, a preference relationship signifies the player's individual assessment of all potential outcomes. In a number of different instances, the preference link is signified by a utility function; this attributes a number to all of the possible outcomes, with higher utilities representing more desirable outcomes. In the wireless MAC scenario, a player might prefer outcomes that yield higher channel utilisation, lower channel access delay, and better fairness, although in many practical situations, these goals will be in conflict. Ultimately, depending on the game's nature, there are a number of different solution concepts that commonly depend on the principle of equilibrium. Using this principle ensures that a player will gain optimal payoff with considering the strategies of other players. One other important game concept is Pareto optimality. A strategy is called Pareto optimal, 
if it is impossible to make one player better off without requiring other players to forfeit or sacrifice in any way possible. The suitable modelling and implementation of such preference relationships is recognised as being one of the most challenging aspect of game theory, which will be reviewed briefly in the following sections.

\subsection{Game Theoretic Models}

When modelling multiple access schemes, there are two types of game that can be used, namely cooperative and noncooperative games. In the case of the former, group rationality behaviour is displayed by players, who implement an enforceable contract within their group; in the case of noncooperative methods, self-interested behaviours are displayed by the players, meaning decisions are made based only on their individual payoff.

\subsubsection{Noncooperative games}

In noncooperative games, players are not able to establish binding commitments [13]; however, this is not to suggest that players are not able to cooperate: in these games, any cooperation needs to be self-enforcing. Such a game has been applied widely with the aim of improving the performance of wireless networks (e.g, various aspects of MAC games, power control in CDMA, time slot competition, etc.). Nash equilibrium [13] is a well-known equilibrium concept in the context of noncooperative games; this is a joint approach where there is no incentive amongst players to deviate from their strategy when considering that other players behave in the same way. This means that a player's utility cannot be enhanced through unilateral deviation. In the case of pure strategies, $s_{i} \in \mathbf{S}_{i}{ }^{9}$ is a Nash equilibrium if the following is true:

$$
u_{i}\left(s_{i}^{*}, \mathbf{s}_{-\mathbf{i}}^{*}\right) \geqslant u_{i}\left(s_{i}, \mathbf{s}_{-\mathbf{i}}^{*}\right), \forall s_{i} \in \mathbf{S}_{\mathbf{i}}, \forall i \in N,
$$

\footnotetext{
${ }^{9} \mathbf{S}_{\mathbf{i}}$ denote a set of strategies of player $i$
} 
where $u_{i}($.$) is the payoff function of player i$; $s_{i}^{*}$ is a Nash equilibrium strategy of player $i$; and $\mathbf{s}_{-\mathbf{i}}^{*}$ is a Nash equilibrium strategy vector of all players except player $i$. The most glaring shortcoming of this equilibrium concept is the fact that it is almost impossible to justify why players in a real game would necessarily play with such equilibrium, and if they did play such an equilibrium, whether it is unique.

Another equilibrium concept for noncooperative approaches is correlated equilibrium; this is recognised as being a more generalised version of the Nash equilibrium [58]. A strategy profile is a correlated equilibrium in situations where players decide upon their strategy in line with the joint distribution as opposed to marginal distribution, as in the case of the Nash equilibrium. Notably, an official definition has been provided: a probability distribution $\pi$ over $\mathbf{S}_{\mathbf{1}} \times \ldots \times \mathbf{S}_{\mathbf{N}}$ is a correlated equilibrium if every strategy $s_{i}^{*} \in \mathbf{S}_{\mathbf{i}}$ satisfies $\pi\left(s_{i}^{*}, \mathbf{S}_{-\mathbf{i}}\right)>0$, and every alternative strategy $\left(s_{i} \in \mathbf{S}_{\mathbf{i}}\right)$ must satisfy $\sum_{s_{-i} \in \mathbf{S}_{-\mathbf{i}}} \pi\left(s_{i}^{*}, \mathbf{S}_{-\mathbf{i}}\right)\left[u_{i}\left(s_{i}^{*}, \mathbf{S}_{-\mathbf{i}}\right)-u_{i}\left(s_{i}, \mathbf{s}_{-\mathbf{i}}\right)\right] \geq 0, \forall s_{i} \in \mathbf{S}_{\mathbf{i}}, \forall i \in N$. In other words, upon a recommendation (i.e., a recommended strategy according to the distribution $\pi$ ) being made to player $i$, a distribution $\pi$ is recognised as a correlated equilibrium if no player $i$ is able to select a strategy $s_{i}$ as opposed to $s_{i}^{*}$, thus causing a higher expected payoff [13]. In terms of classification, a noncooperative game may be considered as an incomplete or complete information game. In the case of the former, this involves players' lack of information of various characteristics relevant to their opponents; such information might relate to their payoff, strategies and/or available actions. On the other hand, complete information games give players all information relating to their opponents. An incomplete information game can be modelled as a Bayesian game [13, 11], which involves Bayesian analysis being applied with the aim of predicting the game's outcome. Furthermore, games may be considered either dynamic or static in nature: the former involves a player selecting an action at the current stage, with consideration to the knowledge of the actions selected by other players, either in previous phases or at the current phase. The latter is 
a one-shot game involving all players making decisions without any knowledge relating to the strategies adopted by other players.

Importantly, in the case of dynamic games, the common equilibrium solution is a subgame perfect Nash equilibrium [59], which can be interpreted as a Nash equilibrium of every sub-game of the original game. One approach utilised widely in order to achieve sub-game perfect equilibria is backward induction.

A dynamic game with incomplete information can be described as a multi-stage game when information is unknown to other players [13]. It is similar to a dynamic game with complete information in that the players take turns sequentially rather than simultaneously, but information is incompletely known by others. The players follow their beliefs and dynamically update their beliefs by using Bayes' rule which is a natural and standard way to handle incomplete information games [13. In a dynamic game with incomplete information, perfect Bayesian equilibrium is the solution concept. The term Bayesian equilibrium is used to refer to a Nash equilibrium in which players update their strategies according to Bayes' rule.

Repeated games are an important tool for understanding concepts of "reputation" and "punishment" in game theory [13]. In a repeated game formulation, players participate in repeated interactions within a potentially infinite time horizon. Players must therefore, consider the effects that their chosen strategy in any round of the game will have on their opponents' strategies in subsequent rounds. Each player tries to maximise its expected payoff over multiple rounds. It is well-known that some single-stage games result in Nash equilibria that are suboptimal from the point of view of all players. The key issue is that each player must consider possible reactions from opponents that will impact that player's future payoffs. For instance, selfish behaviour may be punished. There is a very natural relationship between the notion of a repeated game and that of a Markov game. A Markovian game (i.e., Markovian dynamic game or stochastic game) is an extension 
of game theory to Markov decision process-like environments. In a stochastic or Markov game, the history at each stage of the game can be summarised by a state, and movement from state to state follows a Markov process. In other words, the state at the next round of the game depends on the current state and the current action profile [60, 61, 62].

Lastly, there is the Stackelberg leadership model, also known as leader-follower game [13. This game adopt a strategic approach, in which the player acting as a leader moves first and then the players acting as follower move sequentially. The leadership model is based on establishing an optimal strategy for the leader, presuming that the followers behave rationally and optimise their objective functions in direct consideration of the leader's actions. The Stackelberg game model can be solved through the utilisation of the sub-game perfect Nash equilibrium.

\subsubsection{Cooperative games}

In contrast with noncooperative games, in the case of cooperative games, players can make binding commitments. In this type of game, players maximise their payoffs based on the common objective of a coalition to which they belong. This means, the players are able to implement strategy coordination and agree on the way in which payoff is to be divided amongst all individuals in a coalition. One of the most widely researched cooperative games is Nash bargaining [13], which involves players maximising their gain in consideration to what would be received by each player without cooperation. Essentially, the Nash bargaining solution is defined as following [13]:

$$
\mathbf{s}^{*}=\underset{s}{\operatorname{argmax}} \prod_{i \in N}\left(u_{i}\left(s_{i}\right)-u_{i}^{d}\right)
$$

where $\mathbf{s}^{*}$ is a Nash bargaining solution strategy vector of all players, and $u_{i}^{d}$ is the threat point (i.e., the utility gained if player $i$ decides not to cooperate and bargain with the other players). 


\subsubsection{Challenges in game theoretic design of random channel ac- cess schemes}

As discussed in the introduction, when seeking to model and analyse nodes' cooperative and noncooperative behaviours and their interactions throughout the course of channelaccess contention in the case of wireless networks, game theory may be applied. However, when this is achieved and multiple access schemes in wireless networks are analysed, various considerations are needed to deliver guidance on the suitable application of game theory. This is because, it will help examine the possible future applications and directions of game theory.

- Self-interest and group-rationality: The majority of game theoretic frameworks that are designed for distributed protocols in wireless networks presume group rationality and self-interest in the case of both cooperative and noncooperative game models. A self-interest node (player) only tires to maximise its own payoff function; nevertheless, such behaviours might not be well positioned to deliver a socially ideal solution, which then encourages the implementation of a cooperative game in order to achieve socially optimal solution for all the nodes in the network (e.g., a bargaining game) [63, 64]. However, in most cases, the condition of group rationality might not be true across all nodes, with some showing non-conformity to cooperation. Accordingly, there is the necessity of a penalisation mechanism [65] to ensure cooperation among the nodes is enforced and guarantee the achievement of a socially optimal solution.

- Practical implementation: Despite the fact that the game theory provides solutions for distributed protocols such as DCF, the implementation of such solutions in a practical setting remains problematic. In a number of situations, game-theoretic access methods require information from the channel (e.g., power, SINR) in order 
to convergence to the equilibrium solution, which in reality is impossible to obtain accurately. This means, the stations might need to learn such information from the radio environment with less accuracy, thus potentially this lead to the overall complexity of implementation and accordingly decreasing the rate of convergence to the equilibrium solution.

- Payoff function: A payoff function in the game shows a player's benefit or reward as a strategy is selected by the player. Designing an appropriate payoff function requires considerable insight into the problem at hands. There is a need to define the payoff function in consideration to the nodes' and/or network's physical performance measures (cf. \$3.2.4 and 4.2.2 for designing of payoff functions in Chapter 3 and Chapter 4).

The following section briefly reviews a number of different game-theoretic models proposed in the literature for the study of distributed protocols in wireless networks.

\subsection{Games for Multiple Access Schemes}

There are a number of different game models, such as cooperative, noncooperative, and Stackelberg - all of which used to model and analyse the ALOHA channel access scheme behaviour both with and without rate adaptation and power control. In the majority of the ALOHA game models, nodes are given the option of choosing whether to transmit or not to transmit, with the assumption that their possible actions and nodes' transmission powers are fixed. Tables 2.1 summarize the game models formulated for the Aloha access mechanisms.

There are also a number of different game models, same as ALOHA protocols - all of which used for the analysis of the CSMA/CA-based channel access scheme. Tables 2.2 summarise the game models formulated for CSMA/CA-based access mechanisms. In some CSMA/CA-based MAC game models, the action set of nodes is defined as channel access 
probabilities. In addition, most of the CSMA/CA-based MAC game models consider only the symmetric strategy case by assuming that all nodes are identical and that throughput maximisation is the key objective. In some of the CSMA/CA-based game models, the actions are "To transmit" and "backoff". The solutions of these game models are mixed strategies. Since in random access schemes, nodes access the channel(s) in a distributed manner, some nodes may misbehave. Moreover, to the best of author knowledge, there is no study in the game theoretic literature so far that addresses the hidden and expose terminal problem. This is because, almost all of the studies consider single-cell networks.

\subsection{Summary}

In this chapter, the wireless medium access problem was discussed, and the performance analysis of the node's behaviour in wireless networks, particularly in 802.11 wireless LANs, was briefly reviewed. Then, some designs that propose better contention resolution algorithms that relate to this study to address the shortcomings in the wireless medium were also discussed. It can be observed that the main problem with all of these proposals is related to the core principle of dynamic load control, which implies that these methods cannot distinguish collisions from corrupted frames, nor can they handle the capture effect. In the next chapters, a method similar to [14] and [57] is proposed that relies on only observing idle periods in the channel activity and is therefore insensitive to most of the problems that are discussed in this chapter. The random channel access from the game theory point of view was investigated in this chapter, and a background was provided on how game theory can be applied to a random channel access protocol and the way in which an equilibrium solution (i.e., behaviour of the nodes at steady state) can be obtained. 


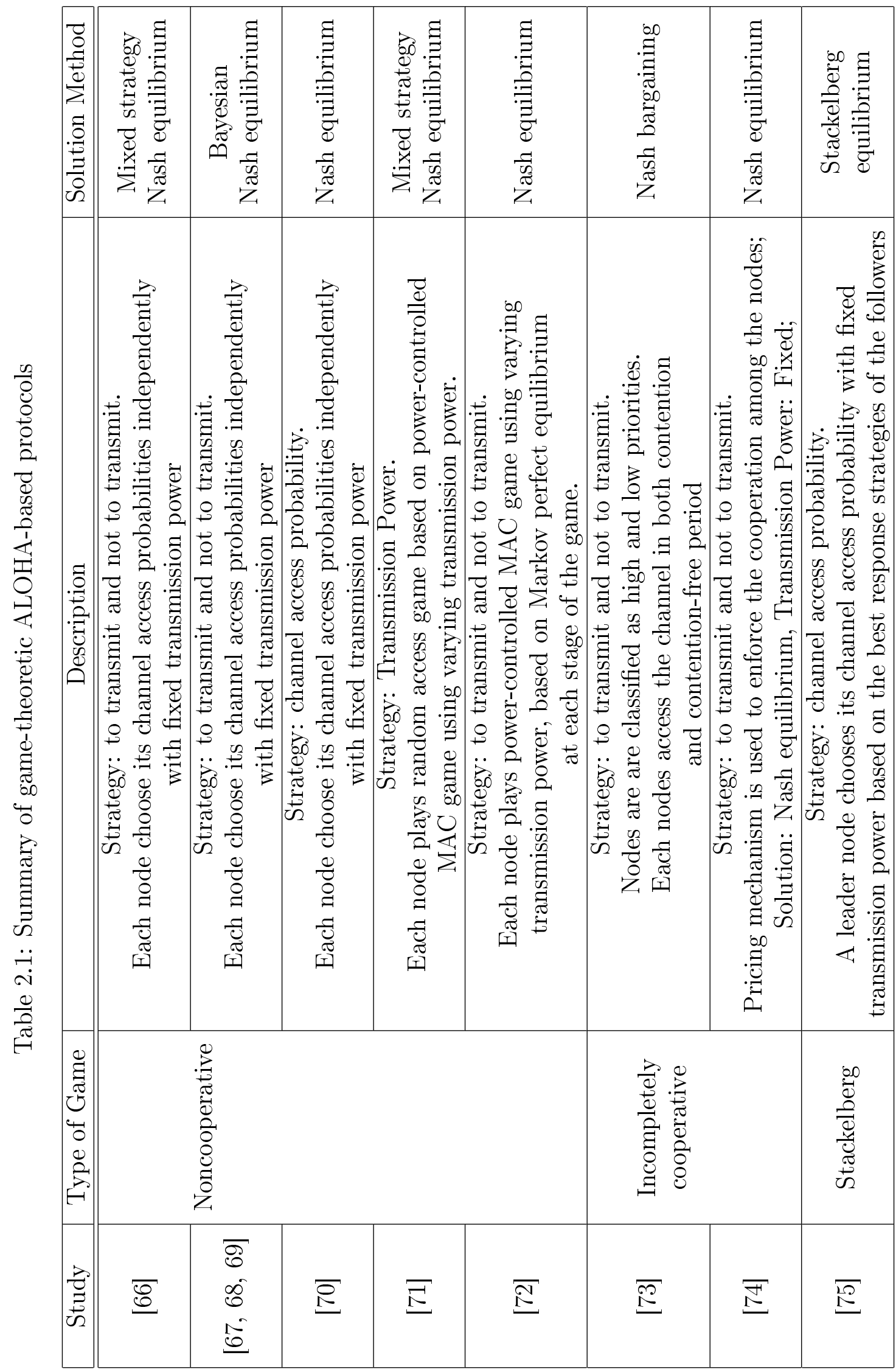




\begin{tabular}{|c|c|c|c|c|c|c|c|c|}
\hline 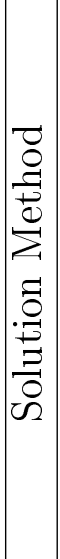 & 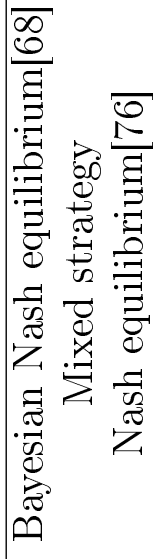 & 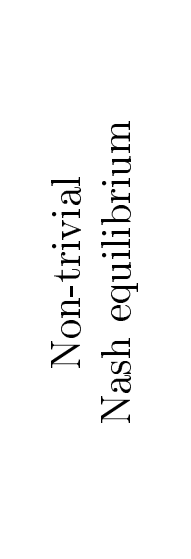 & 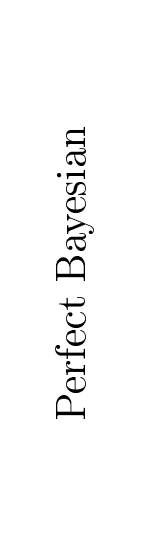 & 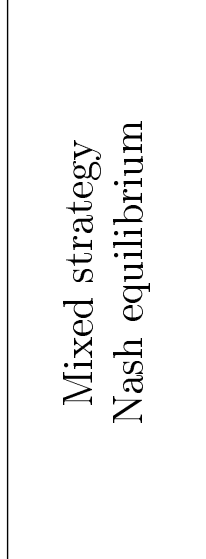 & 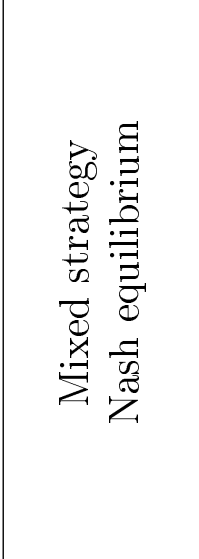 & 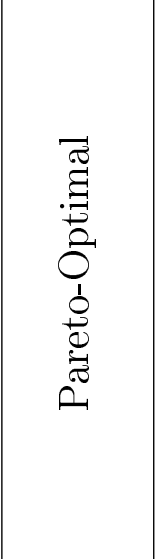 & 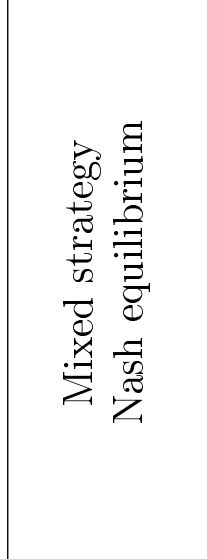 & 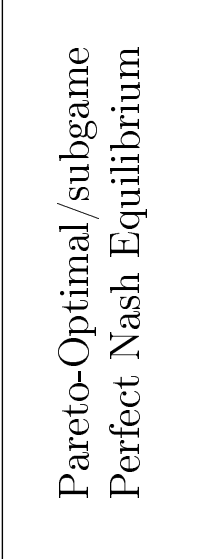 \\
\hline 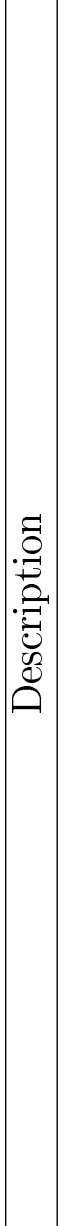 & 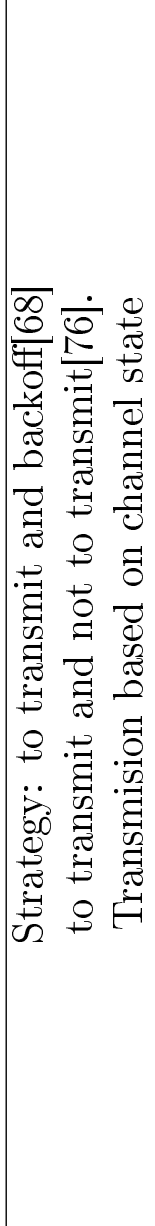 & 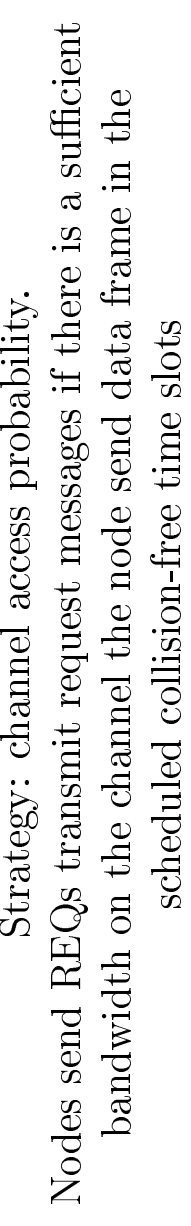 & 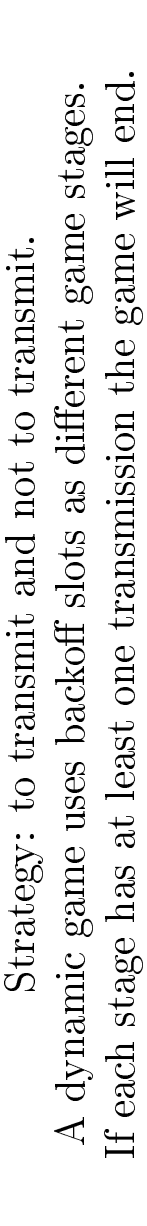 & 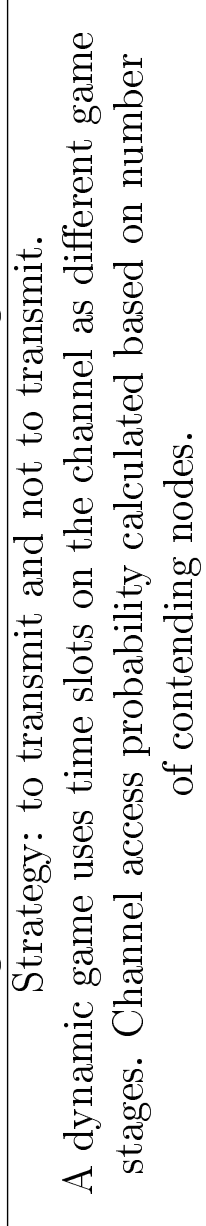 & 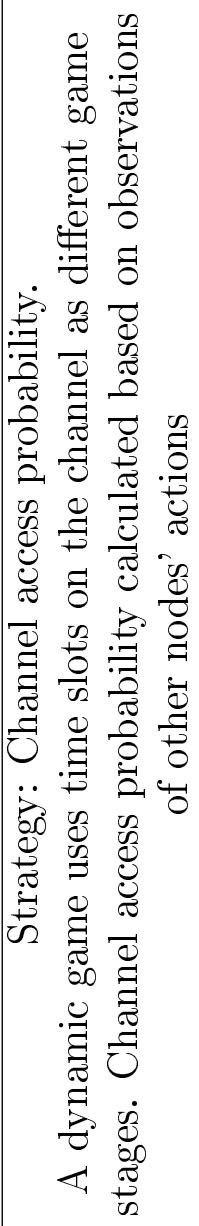 & 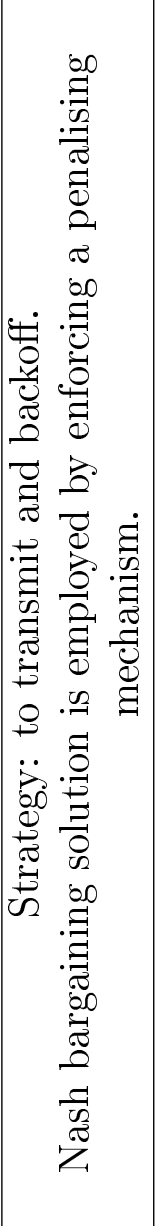 & 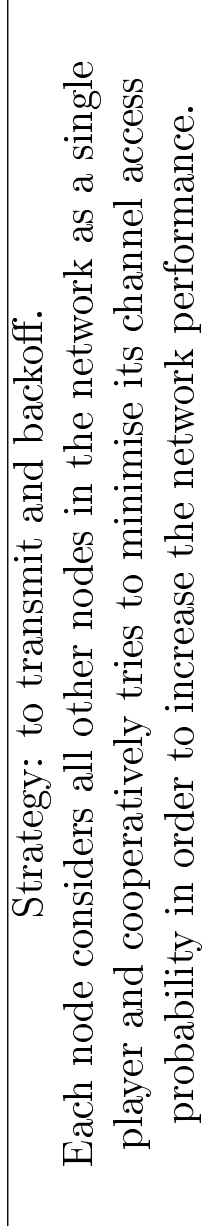 & 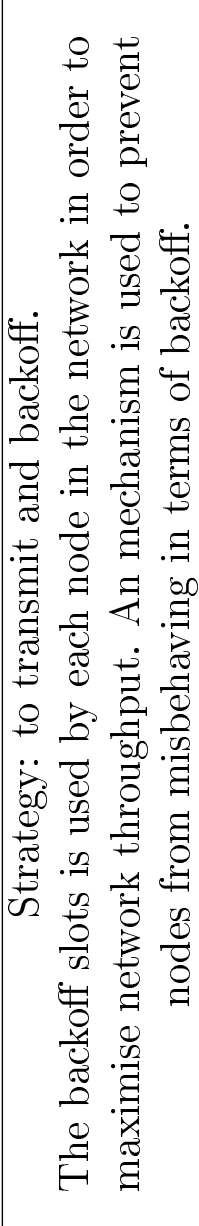 \\
\hline 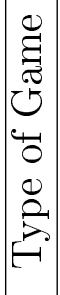 & \multicolumn{2}{|l|}{ 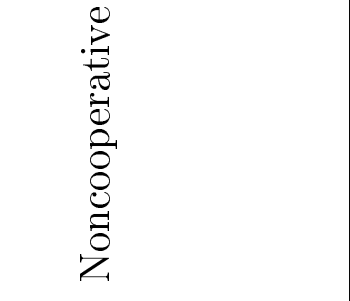 } & \multicolumn{3}{|l|}{ 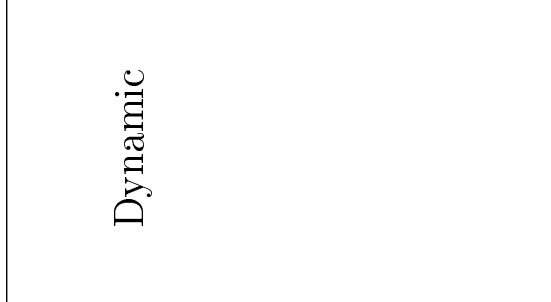 } & \multicolumn{2}{|l|}{ 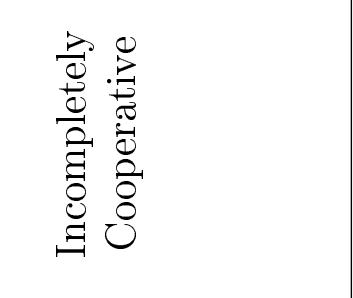 } & 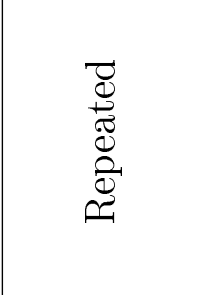 \\
\hline 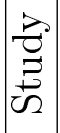 & $\begin{array}{l}0 \\
0 \\
0 \\
0\end{array}$ & $N$ & $\infty$ & $\underline{\underline{p}}$ & 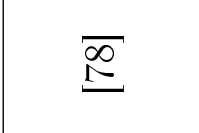 & $\stackrel{2}{2}$ & $\infty$ & $\underline{\infty}$ \\
\hline
\end{tabular}




\section{CHAPTER 3}

\section{Game Theoretic DCF for Single Cell}

\section{WIRELESS NETWORK}

\subsection{Introduction}

This chapter presents a game-theoretic random access model capable of being readily incorporated into the IEEE 802.11 DCF standard. The objective is to design a game theoretic model that can potentially optimize throughput and fairness in each node independently and, therefore, minimise channel access delay. A utility function is proposed, such that it can decouple the protocol's dynamic adaptation to channel load from collision detection by replacing binary exponential backoff mechanism with game theoretic approach for backoff mechanism (cf. \$3.2.2), which ultimately results in constant contention window $(\mathrm{CW})$ for each active node in the network. The proposed model demonstrated that it can reach a Nash equilibrium, which results in a relatively stable contention window provided that each node adapts its behaviour to the idle rate of the broadcast channel, coupled with observation of its own transmission activity.

A noncooperative, static, game-theoretic approach is adapted to model contentionbased wireless medium access: In noncooperative games, the players make rational decisions considering only their individual payoffs. The players are unable to reach an 
enforceable contract, but this does not mean that the players do not cooperate, provided that the game is appropriately designed. However, any cooperation implied by this kind of games must be self-enforcing (cf. 2.4.1). Moreover, due to the nature of the wireless channel, a static game is used, which means that all players make decisions without the knowledge of the strategies that are being chosen by the remaining competing players (cf. \$2.4.1). To design utility and payoff functions, a single cell network is assumed, implying that all nodes can sense each other's transmissions. It is worth mentioning that this protocol can be integrated into DFC for both centralised and ad hoc wireless network topologies.

The idle-sense method is employed to design our utility and payoff functions, as proposed by Heusse et al. [14], which derives the optimal number of consecutive idle time slots between transmissions (dependent on the channel modulation scheme) in order to avoid or minimise contention. Then, gradient play is used as a strategy update mechanism to dynamically control the contention window size by adjusting a current channel access probability gradually in a gradient direction towards the optimal value of the number of idle slots observed between transmission attempts. In Chapter 6 numerical simulations of proposed method in this chapter is shown, including throughput, short-time fairness, collision rate, and channel access delay.

The remainder of this chapter is organized as follows. In $\$ 3.2$, the formulation of a random access game theoretic model is structured by first reviewing the operation of the IEEE 802.11 DCF in 33.2 .1 and then casting the essence of its operation in game theoretic terms by specifying a per-node utility function in $\$ 3.2 .2$. Section 3.2 .3 defines the Nash equilibrium and investigates under what kind of conditions a unique nontrivial Nash equilibrium can be achieved. In $\$ 3.2 .4$, the design of the utility function is specified using reverse engineering from a desired point of operation based on the conditions discussed in $\$ 3.2 .3$. Furthermore, in $\$ 3.2 .5$, a method is proposed based on the game that adjusts 
the CW size much less aggressively than the commonly used binary exponential backoff algorithm. Then in $\$ 3.3$, a MAC protocol is designed, based on the formulated game. Finally, in $\$ 3.4$, a summary of this chapter is given.

\subsection{Formulation of Random Access Model}

As mentioned in Chapter 2, the broad consensus in the published literature is that the performance of DCF is highly dependent on the number of active nodes in the network. Consequently, most of the proposals for improving the IEEE 802.11 MAC attempt to estimate the number of competing stations in the network and adjust the contention window size accordingly (cf. 2.2 .3 . In practice, it is difficult to calculate the number of (active) competing stations accurately because the DCF backoff scheme cannot distinguish collisions from frame corruption events that commonly occur in wireless networks, especially in weak signal areas. Thus, most proposed methods that depend on estimating the number of (active) nodes suffer from the above inaccuracy. In this chapter a channel access method is sought that decouples the channel access probability, $P_{i}^{\tau}$, from the conditional collision probability, $P_{i}^{c}$, in order to provide the ability to discriminate between channel contention and occasional channel lack of reliability. Thus, in order to achieve high throughput and relatively good short-time fairness, a game-theoretic random access model based on CSMA/CA is proposed that provides an appropriate CW size independently for each node in the network. This yields an almost equal channel access probability for all nodes only through local observation of consecutive idle slots between transmissions.

\subsubsection{Description of DCF}

The operation of the DCF channel access mechanism is well-documented in [17]. In order to model the contention resolution algorithm in the context of game theory, this section attempts to explain both basic access and request to send (RTS) / clear to send (CTS) 


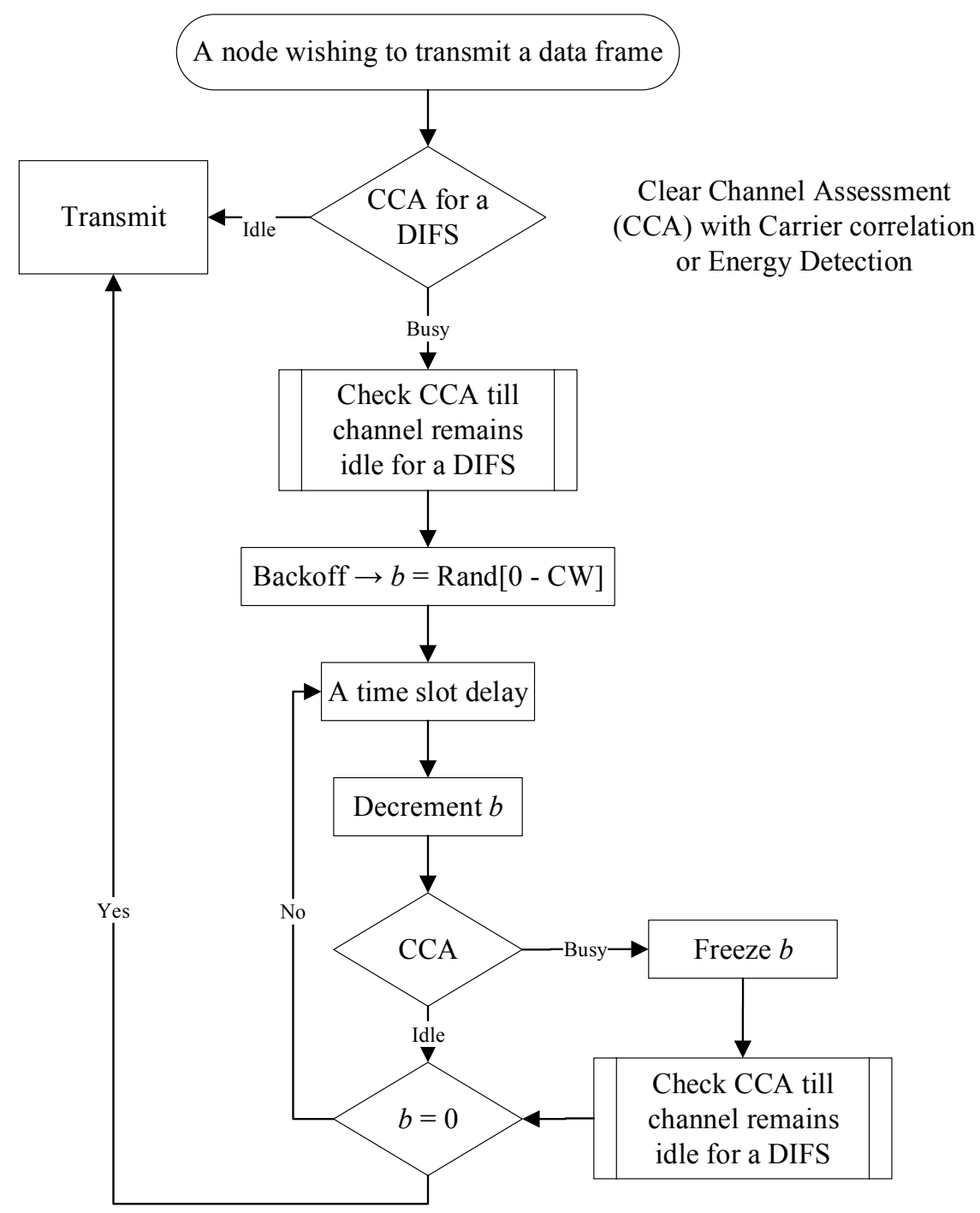

Figure 3.1: Backoff timer operation as a part of collision avoidance mechanism in DCF.

access mechanisms in detail.

A node wishing to transmit, senses the carrier on the channel. If the channel remains idle for a period of time, called a distributed inter frame space (DIFS), the node transmits. Otherwise, the node persists in sensing the channel until this is measured as being idle for a DIFS period. At this point, the node generates a random slotted backoff interval before transmitting. It chooses backoff $b$, an integer distributed uniformly in the window 


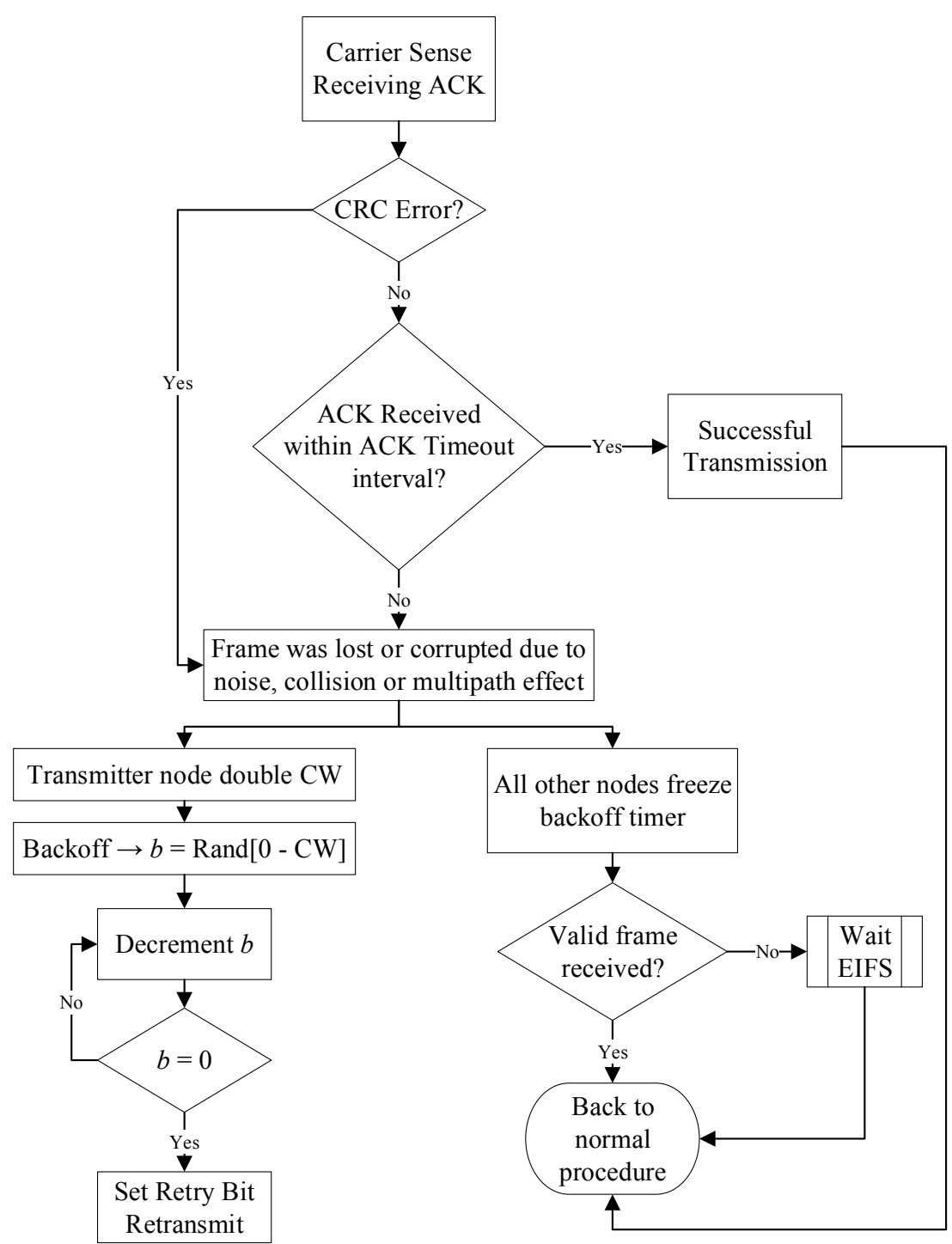

Figure 3.2: Receiving the ACK and CRC check operation.

$[0, C W]$, and waits for period of $b$ time slots before attempting to transmit, while the backoff timer is decremented as long as the channel remains idle. This procedure will be repeated until the node transmits its packet (Figure 3.1). Since the DCF does not rely on the capability of the stations to detect a collision by hearing their own transmission (cf. \$2.1.1), a positive acknowledgement (ACK) is required by the destination station to signal the successful frame reception. Therefore, if the data frame is correctly received, 


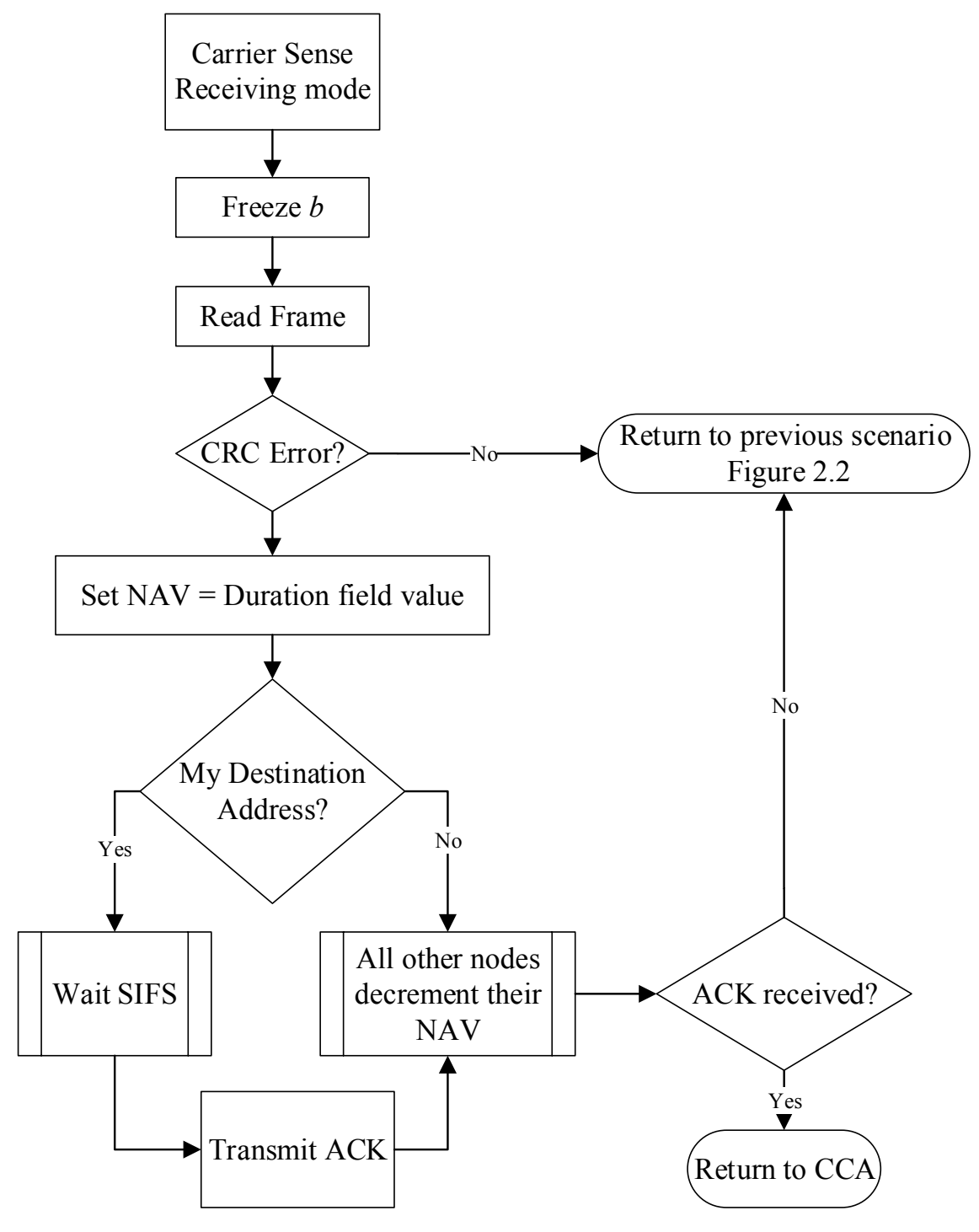

Figure 3.3: Virtual carrier sensing, duration field and the NAV timer.

the receiving node transmits an ACK frame after a fixed period of time, which is called a short inter frame space (SIFS).

Otherwise, if the transmitting node does not receive the ACK frame, it executes the exponential backoff algorithm (it doubles $C W$, which is bounded between $C W_{\text {min }}$ and $\left.C W_{\max }\right)$ and repeats the transmission attempt up to a maximum number of attempts (Figure 3.2). 


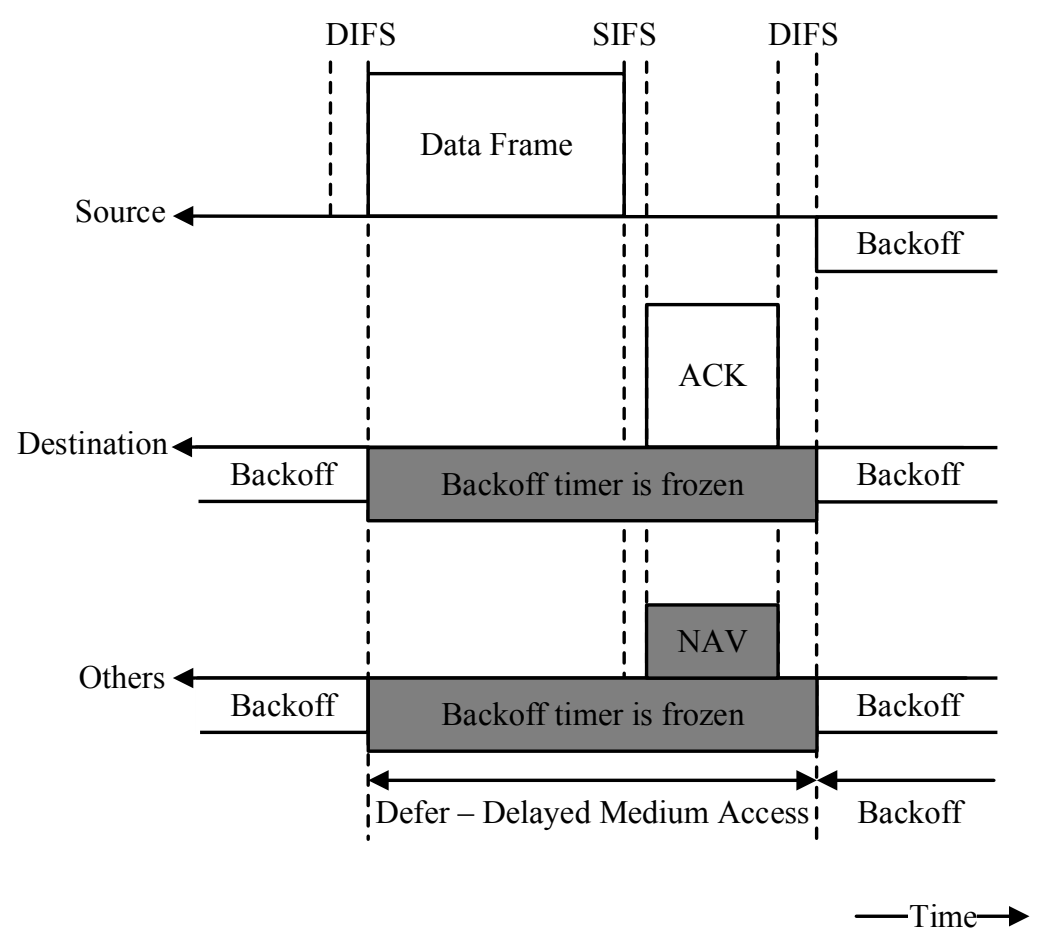

Figure 3.4: Example of basic access mechanism in a single cell wireless network.

It is also unusual for all nodes to be able to communicate directly with all other nodes (cf. \$2.1.2). For this reason, DCF implements a network allocation vector (NAV). The NAV is a value that indicates to a station the amount of time that remains before the medium will become available. In the basic access mechanism, NAV information is implemented using a duration field in the MAC header of each data frame. The value of the duration field specifically represents the transmitter's estimate of how much time is needed to complete the current frame sequence. Even if the medium does not appear to be carrying a transmission via physical carrier sense, a listening station may avoid transmitting. Therefore, the NAV plays the role of virtual carrier-sensing mechanism in DCF (cf. Figure 3.3). By combining the virtual carrier sensing mechanism with the physical carrier sensing mechanism and the binary exponential backoff mechanism, the DCF implements the collision avoidance portion of the CSMA/CA access mechanism. Although 


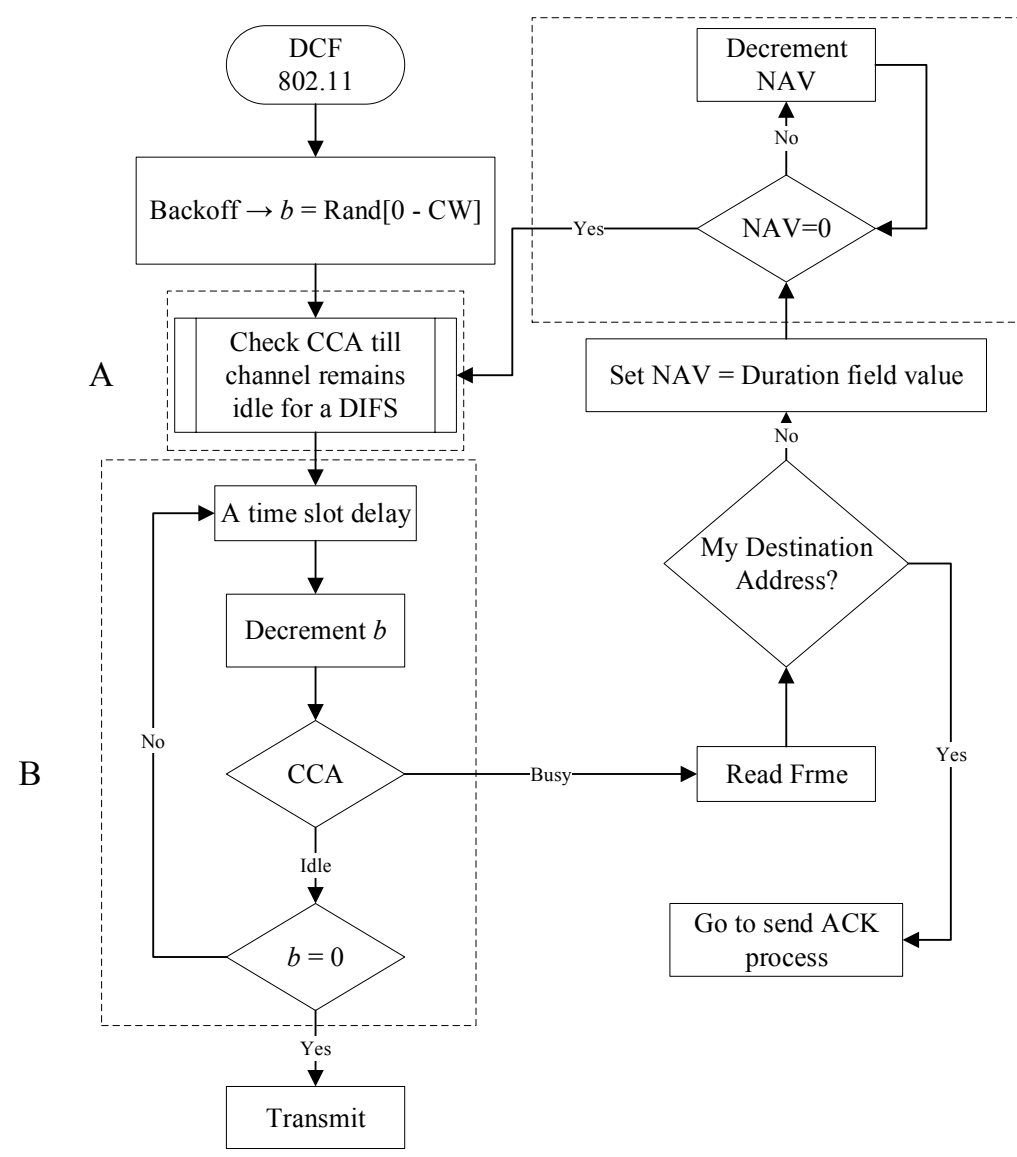

Figure 3.5: The simplified basic access mechanism operation.

both the NAV and the backoff mechanism support collision avoidance, they perform very different functions: e.g., a non-zero NAV timer indicates that virtual carrier sensing has reserved the use of the broadcast medium, but a non-zero backoff timer identifies a node in a randomly ordered queue waiting to transmit. Figure 3.4 illustrates a successful basic access operation in which all stations share the same wireless channel. At the end of the frame transmission by the source, all other stations wait for a DIFS and then choose a random backoff time before transmitting another frame. Note that the frame transmission by the source occurs in the middle of the backoff time for the destination node. As a consequence of the channel being sensed as busy, the backoff time is frozen, and the 


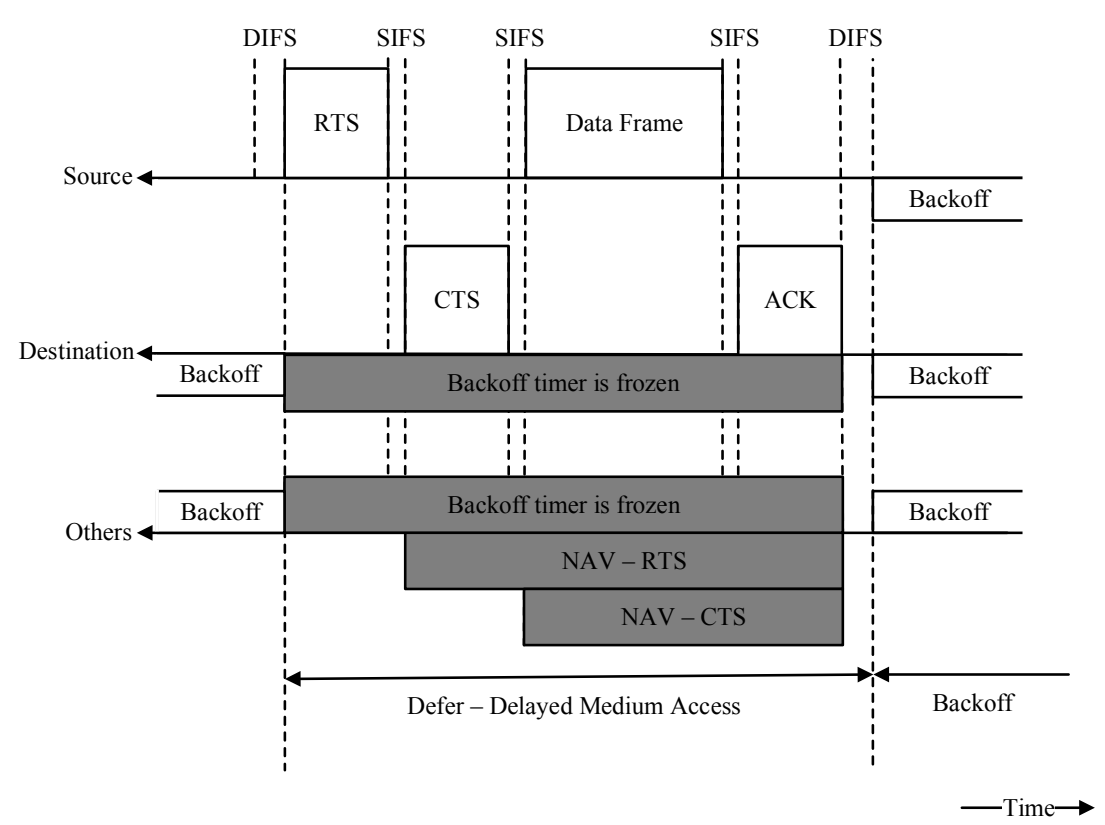

Figure 3.6: Example of RTS/CTS mechanism in a single-cell wireless network.

backoff counter decrements again only when the channel is sensed idle for a DIFS. The ACK is immediately transmitted at the end of the frame transmission, after a SIFS. As the SIFS (plus the propagation delay) is shorter than a DIFS, no other station is able to detect the channel idle for a DIFS until the end of the ACK transmission.

A simplified version of two-way handshaking technique (the basic access mechanism operation in the DCF), which is described above, is illustrated in Figure 3.5. Once again note that DCF is built based on CSMA/CA and has three main elements: Element A is a physical carrier sense mechanism that works using CCA. Element B and C are a binary backoff and virtual carrier sense respectively, which provide collision avoidance ability for nodes.

DCF also has an additional four-way handshaking technique that is optional. This mechanism is known as request to send / clear to send (RTS/CTS). An example of successful frame transmission is shown in Figure 3.6. A node wishing to transmit senses the carrier on the channel. If the channel remains idle for a DIFS, the node transmits a special short 
frame called request to send (RTS). When the destination station detects an RTS frame, it responds after a SIFS with a clear to send (CTS) frame. The source is allowed to transmit its data frame only if the CTS frame is correctly received. The frames RTS and CTS carry the information of the length of the frame to be transmitted (duration field). This information can be read by any listening station, which is then able to update a network allocation vector (NAV) containing the information of the period of time in which the channel will remain busy. This method is suitable to address the hidden terminal problem. The importance of this method in designing the utility function for a network with hidden nodes will be discussed in next chapter, where a network with hidden terminals is assumed. Note that the RTS/CTS mechanism is exactly the same as the basic access mechanism in terms of collision avoidance except that the NAV information is carried by a RTS and CTS frame. This method provides better time synchronization among the nodes, especially when the size of data frame is large.

\subsubsection{The game}

Now consider a set of wireless network nodes $N$ with DCF as the contention protocol. Associated with each wireless node $i \in N$ is its channel access probability, $P_{i}^{\tau}(t)$, and a certain contention measure observed by each node through the backoff mechanism. As stated in the description of DCF, each node in the network can observe its own channel access probability $P_{i}^{\tau}(t)$ and its conditional collision probability (contention measure), $P_{i}^{c}(t)$, but not those of other nodes. It can then adjust its channel access probability, $P_{i}^{\tau}(t)$, based on a system control point of view, written as:

$$
P_{i}^{\tau}(t+1)=\mathcal{F}_{i}\left(P_{i}^{\tau}(t), P_{i}^{c}(t)\right)
$$

where time is slotted in units of a slot duration. The conditional collision probability depends on the channel access probability, represented through a vector $\mathbf{P}^{\tau}(t)=\left(P_{i}^{\tau}(t), i \in\right.$ 
$N):$

$$
P_{i}^{c}(t)=\mathcal{C}_{i}\left(\mathbf{P}^{\tau}(t)\right)
$$

Here, $\mathcal{F}_{i}$ models the contention resolution algorithm, and $\mathcal{C}_{i}$ models a mechanism that updates the conditional collision probability in DCF. It is assumed that Equation 3.2.1 and 3.2.2 have an equilibrium $\left(\mathbf{P}^{\tau}, \mathbf{P}^{\mathbf{c}}\right)$. The fixed point of Equation 3.2.1 defines an implicit relation between the equilibrium $P_{i}^{\tau}(t)$ and $P_{i}^{c}(t)$. Then, by the implicit function theorem [82], there exists a unique continuously differentiable function $F_{i}$, such that,

$$
P_{i}^{c}=F_{i}\left(P_{i}^{\tau}\right)
$$

In [9] the probability $P_{i}^{\tau}$ that a station transmits in a randomly chosen slot time when the backoff time counter is equal to zero, regardless of the backoff stage, is expressed as the following:

$$
P_{i}^{\tau}=\frac{2\left(1-2 P_{i}^{c}\right)}{\left(1-2 P_{i}^{c}\right)(C W+1)+P_{i}^{c} C W\left(1-\left(2 P_{i}^{c}\right)^{m}\right)}
$$

where $m$ is the backoff stage. Also, the probability that a transmitted packet encounters a collision (the conditional collision probability) is the probability that, in a time slot, at least one of the remaining stations in the network transmits. Therefore this probability is defined as the following:

$$
\mathcal{C}_{i}\left(\mathbf{P}^{\tau}\right)=1-\left(1-P_{i}^{\tau}\right)^{N-1}, \forall i \in N
$$

The contention window $(\mathrm{CW})$ in DCF is formulated in terms of the node channel access probability, $P_{i}^{\tau}$ (cf. Equation 3.2.4). When, $m=0$, i.e., no exponential backoff is considered, the CW easily maps to a backoff mechanism by the following correspondence between $P_{i}^{\tau}$ and a constant contention window, that holds in saturation mode [9, 35]. The 
saturation mode implies that every node has a packet ready to transmit at all times.

$$
P_{i}^{\tau}=\frac{2}{C W+1}
$$

Therefore, the probability $P_{i}^{\tau}$ is independent of $P_{i}^{c}$ (c.f. Equation 3.2.6). However, the current backoff scheme in DCF considers the lack of an ACK reception as a collision and doubles its contention window, which is the main source of short-time unfairness [48]. Therefore, a constant contention window is adopted in our utility function design in order to preserve the independence of $P_{i}^{\tau}$ from $P_{i}^{c}$. As will become clear later, such a choice of constant contention window in the protocol plays a crucial role in guaranteeing the uniqueness of the Nash equilibrium and enabling the design of a payoff function. Therefore, the utility function of each node $i$ is defined as follows:

$$
U_{i}\left(P_{i}^{\tau}\right)=\int_{v_{i}}^{P_{i}^{\tau}} F_{i}\left(P_{i}^{\tau}\right) d P_{i}^{\tau} .
$$

Based on the Lack of dependency of $P_{i}^{\tau}$ on $P_{i}^{c}$ embodied in Equation 3.2.6 and the utility function 3.2.7, a random access game $G$ is defined as a triple $G=\left\{N,\left(S_{i}\right)_{i \in N},\left(u_{i}\right)_{i \in N}\right\}$, where $N$ is the set of players (active nodes in the network). Player $i \in N$, adopts strategy $S_{i}=\left\{P_{i}^{\tau} \mid P_{i}^{\tau} \in\left[v_{i}, P_{i}^{\tau}\right]\right\}$ with $0<v_{i} \leqslant P_{i}^{\tau}<1$, and a payoff function:

$$
u_{i}\left(\mathbf{P}^{\tau}\right)=U_{i}\left(P_{i}^{\tau}\right)-P_{i}^{\tau} \mathcal{C}_{i}\left(\mathbf{P}^{\tau}\right)
$$

with a utility function $U_{i}\left(P_{i}^{\tau}\right)$ and price function $P_{i}^{\tau} \mathcal{C}_{i}\left(\mathbf{P}^{\tau}\right)$. Note that the throughput and fairness of node $i$ depend on the $P_{i}^{\tau}$ (as throughput is proportional to $P_{i}^{\tau}$, if there are no collisions in the network). $P_{i}^{\tau} \mathcal{C}_{i}\left(\mathbf{P}^{\tau}\right)$ is considered as a contention price for node $i$ for a given $P_{i}^{\tau}$. Therefore, potentially each node can calculate precisely the cost of its action based on $P_{i}^{\tau}$, and with the knowledge that $P_{i}^{\tau}$ and $\mathcal{C}_{i}\left(\mathbf{P}^{\tau}\right)$ are independent of 
each other, each node has the ability to maximise its payoff function regardless of having direct knowledge of any other player's action at a specific time. In the next section, we discuss the condition for which our payoff function can produce a unique nontrivial Nash equilibrium and thus all nodes can independently reach almost the same channel access probability, which can then maximise the overall network performance. Here is the list of the assumptions that will be used in the proposed game theoretic approach.

- Assumption 1: The utility function $U_{i}\left(P_{i}^{\tau}\right)$ is twice continuously differentiable and strictly concave and increasing.

- Assumption 2: Function $P_{i}^{i d l e}\left(\mathbf{P}^{\tau}\right)=\left(1-P_{i}^{\tau}\right)\left(1-U_{i}^{\prime}\left(P_{i}^{\tau}\right)\right), \forall i \in N$ at a nontrivial equilibrium $\mathbf{P}^{\tau *}$ is strictly monotone in $S_{i}, \forall i \in N$, which means it is strictly increasing or strictly decreasing function.

Assumption 1 is a standard assumption in economics and can also be seen as derived from those assumptions on Equation 3.2.3. Under this assumption, there exists a Nash equilibrium for any random access game $G$. Assumption 2 guarantees the uniqueness of nontrivial Nash equilibrium, which we will define later in Section 3.2.4.

\subsubsection{Nash Equilibrium}

The objective in this noncooperative game model is to find the equilibrium solution for a wireless network with self-interested nodes in a distributed manner. As mentioned in 2.4.1, a well-known solution for noncooperative games is the Nash equilibrium [13], which jointly maximises all the players' utilities.

Based on the game defined in Section 3.2.1, $\mathbf{P}^{\tau *}$ is Nash equilibrium if it satisfies the following condition [13]:

$$
u_{i}\left(P_{i}^{\tau *}, \mathbf{P}_{-i}^{\tau *}\right) \geqslant u_{i}\left(P_{i}^{\tau}, \mathbf{P}_{-i}^{\tau *}\right), \forall i \in N, \forall P_{i}^{\tau} \in S_{i}
$$


where $u_{i}($.$) is the payoff function of player i$, and $\mathbf{P}_{-i}^{\tau *}$ is a Nash equilibrium strategy vector for all the players except player $i\left(\mathbf{P}_{-i}^{\tau} \equiv\left(P_{1}^{\tau}, \ldots, P_{i-1}^{\tau}, P_{i+1}^{\tau}, \ldots, P_{N}^{\tau}\right)\right)$.

Equation 3.2 .9 states that each player $i$, in choosing $P_{i}^{\tau *}$ is playing its best response to all the other players' strategy choices. A Nash equilibrium $\mathbf{P}^{\tau *}$ is a nontrivial equilibrium if $P_{i}^{\tau *}$ satisfies the following condition [13]:

$$
\frac{\partial u_{i}\left(P_{i}^{\tau *}, \mathbf{P}_{-i}^{\tau *}\right)}{\partial P_{i}^{\tau}}=0, \forall i \in N
$$

Note that the equilibrium condition in Equation 3.2 .10 does not guarantee that the equilibrium is unique and implies that, at the Nash equilibrium, $P_{i}^{\tau *}$ can take values at the boundaries of the strategy space which results in poor performance in terms of throughput and fairness (cf. the assumptions in 3.2 .2 . However, if it satisfies Equation 3.2.11 then the equilibrium $P_{i}^{\tau *}$ is guaranteed to be a unique nontrivial equilibrium [13]:

$$
\frac{\partial U_{i}\left(P_{i}^{\tau *}\right)}{\partial P_{i}^{\tau}}=\mathcal{C}_{i}\left(\mathbf{P}^{\tau *}\right), \forall i \in N
$$

In order to meet the condition in Equation 3.2.11, the utility function $U_{i}\left(P_{i}^{\tau}\right)$ must be continuously differentiable and strictly concave. Since Equation 3.2 .7 is an integral, and since $P_{i}^{c}=F_{i}\left(P_{i}^{\tau}\right)$ and $P_{i}^{c} \geqslant 0, F_{i}\left(P_{i}^{\tau}\right)$ is a continuous and decreasing function (the larger the channel access probability, the smaller the conditional collision probability (cf. Equation 3.2.5, it follows that $U_{i}\left(P_{i}^{\tau}\right)$ is a nondecreasing function: This implies that $U_{i}\left(P_{i}^{\tau}\right)$ is strictly concave. Therefore, $u_{i}\left(\mathbf{P}^{\tau}\right)$ is continuous and concave in $P_{i}^{\tau}$. Hence, there exists a nontrivial Nash equilibrium for game $G$ [13].

\subsubsection{Utility function design}

Choosing an appropriate utility function requires considerable insight into the problem at hand. The utility function in this study is formulated for each node in the network in 


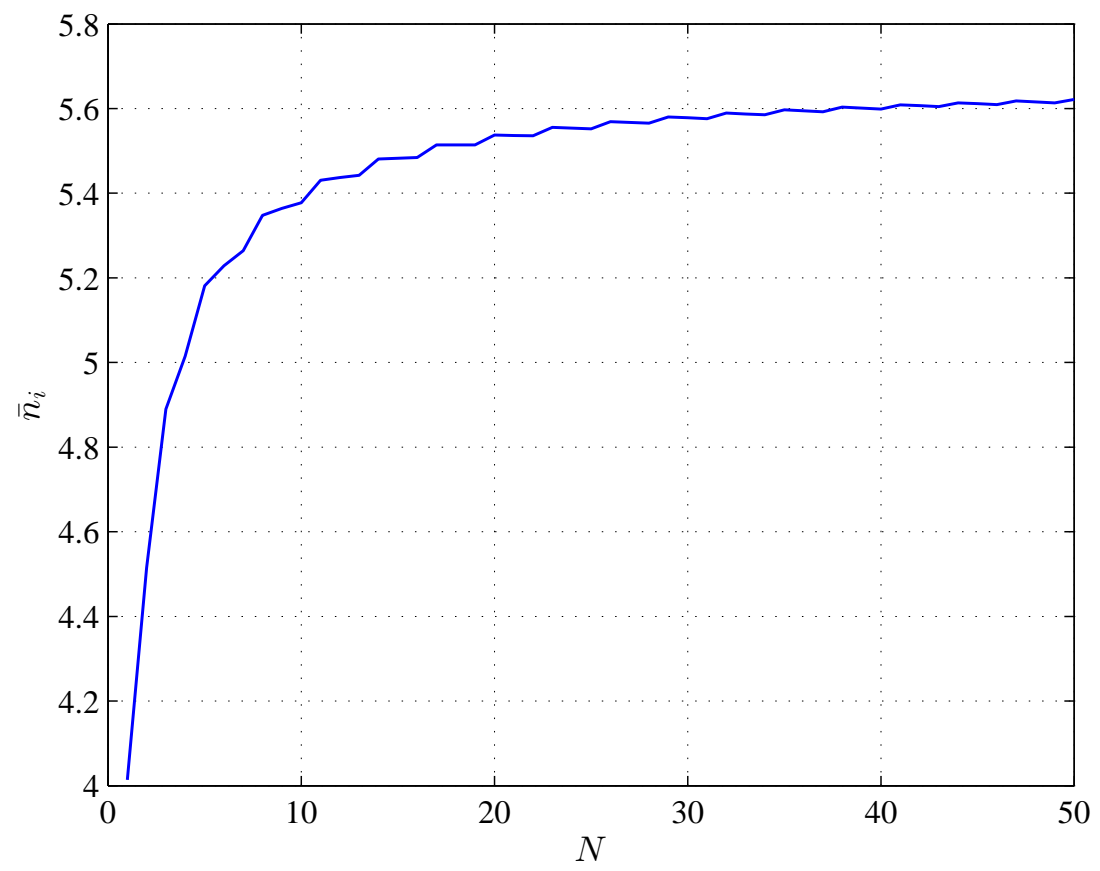

Figure 3.7: Number of nodes versus optimal number of idle slots $\bar{n}_{i}^{\text {opt }}$ for basic access mechanism, up to 50 active nodes based on the parameters in table $3.1(802.11 / \mathrm{b})$.

such a manner as to ensure that its design results in a unique, nontrivial Nash equilibrium for each node. Based on the idle sense method [14], DCF is reverse engineered from the desired operation point. In [14], a medium access method is proposed that uses the mean number of consecutive idle slots between two transmission attempts. Let us denote by $\bar{n}_{i}$ the mean number of consecutive idle slots between two transmission attempts (successful transmission, or collision, or frame corruption). This can be written as a function of $P_{i}^{\text {idle }}$ ( the probability of channel being idle), as follows,

$$
\bar{n}_{i}=\frac{P_{i}^{\text {idle }}\left(\mathbf{P}^{\tau}\right)}{1-P_{i}^{i d l e}\left(\mathbf{P}^{\tau}\right)} .
$$

Under the assumption of a Poisson arrival process, the channel idle probability is [9, 14],

$$
P_{i}^{i d l e}\left(\mathbf{P}^{\tau}\right)=\left(1-P_{i}^{\tau}\right)^{N}
$$




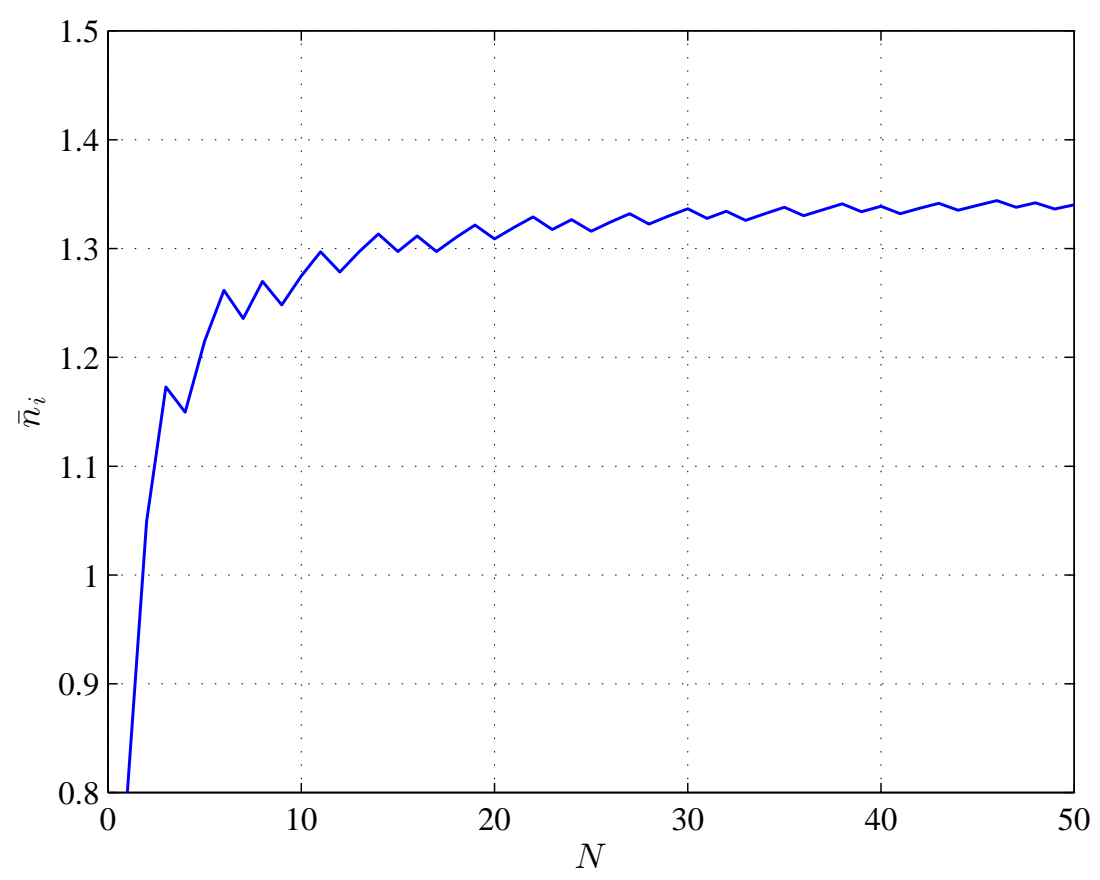

Figure 3.8: Number of nodes versus optimal number of idle slots $\bar{n}_{i}^{\text {opt }}$ for RTS/CTS access mechanism, up to 50 active nodes based on the parameters in table $3.1(802.11 / \mathrm{b})$.

It can be shown using [14] that, provided an equal contention window is used for all competing nodes in a network as $N \rightarrow \infty$, this leads to the following:

$$
P_{i}^{\text {idle(opt) }}=(1-\xi / N)^{N} \rightarrow e^{-\xi}
$$

where $\xi$ satisfies $1-\xi=\eta e^{-\xi}, \eta=1-T_{s} / T_{c}, T_{s}$ is a slot duration that is a fixed period of time, and $T_{c}$ is the average collision duration. Thus, the optimal number of idle slots between two transmission attempts becomes the following:

$$
\bar{n}_{i \infty}^{\mathrm{opt}}=\frac{e^{-\xi}}{1-e^{-\xi}}
$$

If DCF does not use a RTS/CTS handshake scheme, then the average collision duration 


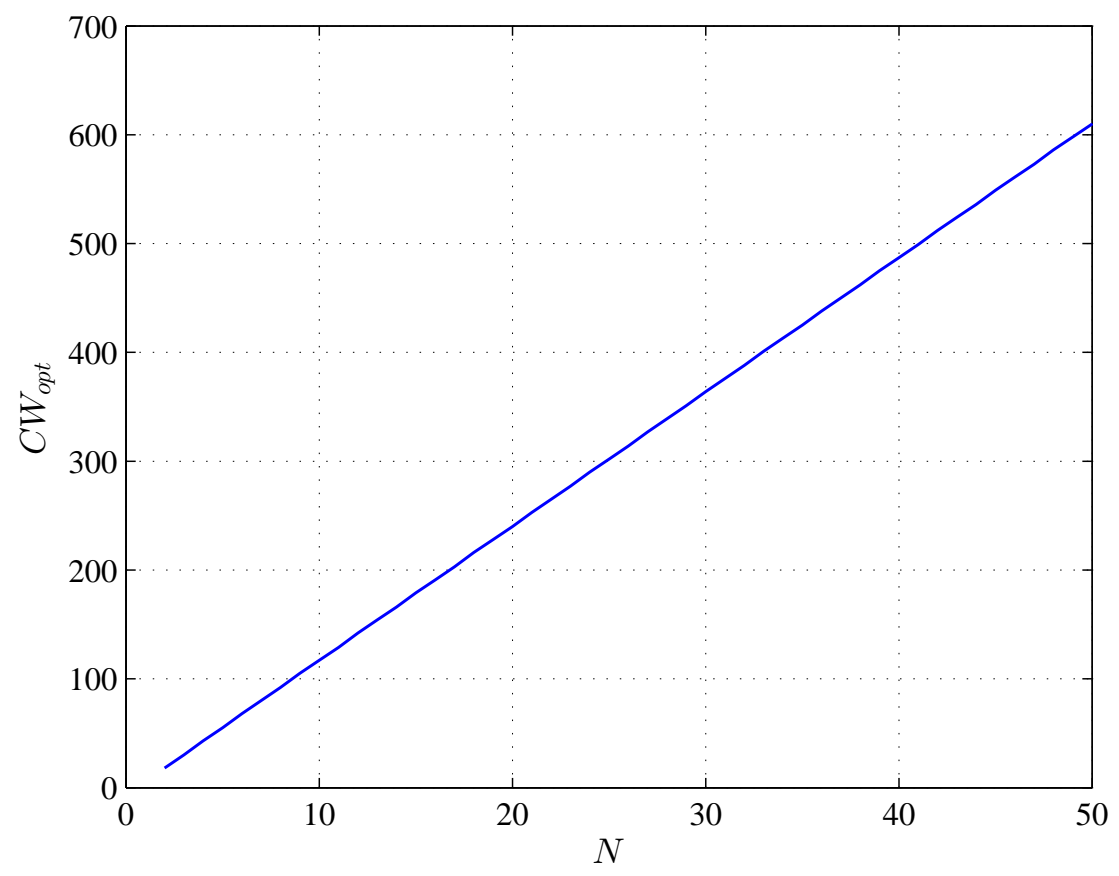

Figure 3.9: Number of nodes versus optimal values of contention window size $C W_{\text {opt }}$ for basic access mechanism based on the parameters in table $3.2(802.11 / \mathrm{b})$.

becomes this:

$$
T_{c}=\frac{P H Y_{h d r}}{b r}+\frac{M A C_{h d r}+L}{v}+D I F S+\widehat{\sigma}
$$

otherwise,

$$
T_{c}=R T S+D I F S+\widehat{\sigma},
$$

where $P H Y_{h d r}$ is the header of the physical layer, and $M A C_{h d r}$ is the header of the MAC layer. $L$ is the packet length/size, $v$ is the channel bit rate, $b r$ is the basic rate and $\widehat{\sigma}$ is the mean propagation delay. Note that $\bar{n}_{i \infty}^{\mathrm{opt}}$ is completely determined by the protocol parameters and not by estimating the number of nodes in the network (Equations 3.2 .12 and 3.2 .13 give the optimal number of idle slots between two transmission attempts $\bar{n}_{i}^{\text {opt }}$ and thus the optimal contention window size $C W_{\text {opt }}$ which are shown in

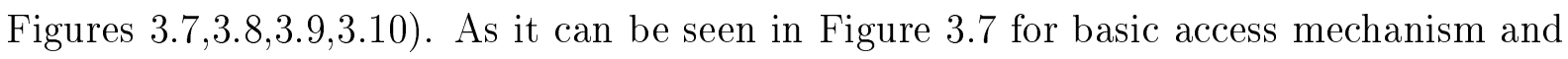




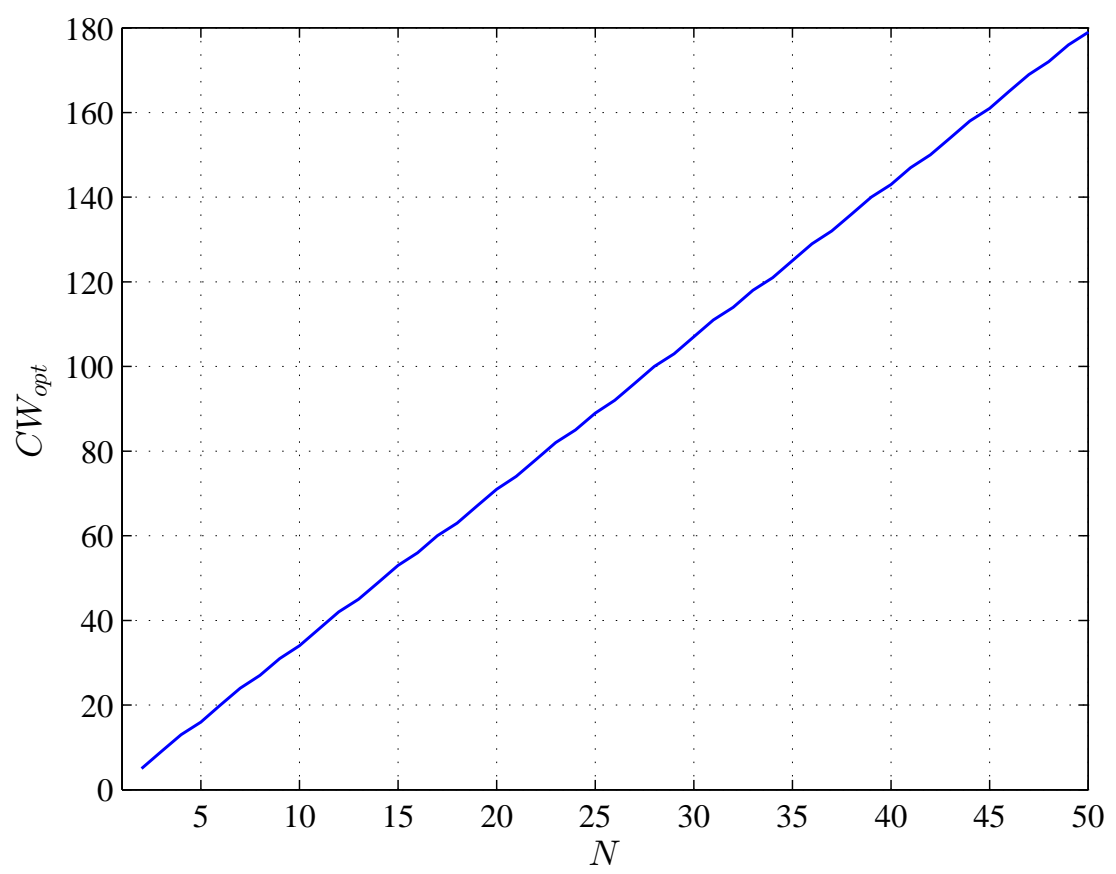

Figure 3.10: Number of nodes versus optimal values of contention window size $C W_{\text {opt }}$ for RTS/CTS access mechanism based on the parameters in table 3.1 $(802.11 / \mathrm{b})$.

Figure 3.8 for RTS/CST access mechanism that as the number of competing nodes increases, the optimal number of idle slots converges quickly to $\bar{n}_{i \infty}^{\mathrm{opt}}$. This indicates that any deviation from $\bar{n}_{i \infty}^{\text {opt }}$ will result in decreasing the network performance in terms of both throughput and fairness. Therefore, without loss of generality, for a large number of nodes $(N \geqslant 10)$,

$$
P_{i}^{i d l e(\mathrm{opt})} \approx e^{-\xi}, \forall i \in N
$$

In order to reverse engineer the utility function from the desired point of operation, it can be shown that,

$$
P_{i}^{i d l e}\left(\mathbf{P}^{\tau}\right)=\left(1-P_{i}^{\tau}\right)\left(1-U_{i}^{\prime}\left(P_{i}^{\tau}\right)\right), \forall i \in N
$$

Therefore, according to Equation 3.2.18, the utility function at its maximum should be $P_{i}^{i d l e}\left(\mathbf{P}^{\tau}\right)=e^{-\xi}$. By applying Equation 3.2.7, the utility function is obtained as the 
Table 3.1: DSSS system parameters and additional parameters used to obtain $\bar{n}_{i}^{\text {opt }}$

\begin{tabular}{|c||c|}
\hline Channel Bit Rate & $11 \mathrm{Mbps}$ \\
\hline Basic Rate & $1 \mathrm{Mbps}$ \\
\hline Slot Time & $20 \mu \mathrm{s}$ \\
\hline DIFS & $50 \mu \mathrm{s}$ \\
\hline SIFS & $10 \mu \mathrm{s}$ \\
\hline Propagation Delay & $1 \mu \mathrm{s}$ \\
\hline Physical Header & $192 \mathrm{bits}$ \\
\hline MAC Header & $272 \mathrm{bits}$ \\
\hline ACK & $112 \mathrm{bits}$ \\
\hline Packet Payload & $12000 \mathrm{bits}$ \\
\hline
\end{tabular}

following:

$$
U_{i}\left(P_{i}^{\tau}\right)=P_{i}^{\tau}+e^{-\xi} \ln \left(1-P_{i}^{\tau}\right)
$$

However, utility function 3.2 .20 is not a strictly nondecreasing function, and hence, it cannot guarantee the uniqueness of the Nash equilibrium. In other words, it does not satisfy assumption 2 in $\$ 3.2 .2$. A convenient approximate choice that satisfies the uniqueness of the Nash equilibrium is as follows:

$$
P_{i}^{\text {idle }}\left(\mathbf{P}^{\tau}\right)=e^{-\xi}\left(1+P_{i}^{\tau}\right)
$$

As mentioned before when $N$ is large, the optimal $P_{i}^{\tau}$ that maximises the throughput is very small [14, 9]. Applying Equation 3.2.7, the utility function is derived as follows:

$$
U_{i}\left(P_{i}^{\tau}\right)=\left(1+e^{-\xi}\right) P_{i}^{\tau}+2 e^{-\xi} \ln \left(1-P_{i}^{\tau}\right)
$$

Therefore, the payoff function now becomes:

$$
u_{i}\left(\mathbf{P}^{\tau}\right)=\left(1+e^{-\xi}\right) P_{i}^{\tau}+2 e^{-\xi} \ln \left(1-P_{i}^{\tau}\right)-P_{i}^{\tau} \mathcal{C}_{i}\left(\mathbf{P}^{\tau}\right) .
$$




\subsubsection{Dynamics of random access game}

This section discusses how interacting players converge to a Nash equilibrium. Since a noncooperative, static, game-theoretic approach is adopted to model contention-based wireless medium access, repeated play of the random access game was considered as an update mechanism in which players adjust strategies in response to observations of other player actions, so as to achieve the Nash equilibrium. The simplest strategy update mechanism is the best response. At each stage, every node chooses the best response to the actions of all the other nodes in the previous round by maximising Equation 3.2.23. Mathematically, at stage $t+1$, node $i \in N$ chooses a channel access probability derived from stage $t$. Then each node $i \in N$ updates its contention window $C W_{i}$ as follows:

$$
C W_{i}(t+1)=\frac{2-P_{i}^{\tau}(t+1)}{P_{i}^{\tau}(t+1)} .
$$

Equation 3.2.24 is derived from Equation 3.2.6 with the assumption that if the size of contention window remains constant, $P_{i}^{\tau}$ and $P_{i}^{c}$ become independent of each other. This relation is extremely accurate when we have a large number of active nodes in the network $(N \geqslant 10)$, as validated through extensive simulations by [83, 9]. Clearly, if the above game reaches a steady state, then this state is a Nash equilibrium. Nonetheless, to the best of this author's knowledge, there are no known convergence results for general games using this approach. We thus consider an alternative strategy update mechanism called gradient play [84]. Each node $i \in N$ updates its strategy according to the following:

$$
P_{i}^{\tau}(t+1)=P_{i}^{\tau}(t)+E\left(\mathbf{P}^{\tau}\right)\left[U_{i}^{\prime}\left(P_{i}^{\tau}(t)\right)-\mathcal{C}_{i}\left(\mathbf{P}^{\tau}(t)\right)\right]
$$

where $E\left(\mathbf{P}^{\tau}\right)>0$ is step size towards the equilibrium The gradient play admits a nice economic interpretation if the conditional collision probability $\mathcal{C}_{i}\left(\mathbf{P}^{\tau}(t)\right)$ is interpreted as 
contention price for node $i$ : If the marginal utility $U_{i}^{\prime}\left(P^{\tau}{ }_{i}(t)\right)$ is greater than the contention price, we increase the channel access probability. Conversely, if the marginal utility is less than the contention price, then the access probability is decreased; then using Equation 3.2.24, each node updates its strategy. The step size determines the convergence speed. In the proposed game, we choose a constant step size $E\left(\mathbf{P}^{\tau}\right)$ for all nodes because the same payoff function is used for all nodes that make the network homogeneous. Moreover, the assumed network is single-cell, which means all nodes can see each other. Therefore, the same step size in a homogeneous single-cell network can guarantee the convergence of the whole network in almost a fixed number of transmissions.

\subsection{Medium Access Control Design}

Based on Section 3.2, a medium access model adopted by each node in a wireless network was designed, instead of using a binary exponential backoff scheme. Hence, each node computes locally its displacement from equilibrium using $U_{i}^{\prime}\left(P^{\tau}{ }_{i}(t)\right)-\mathcal{C}_{i}\left(\mathbf{P}^{\tau}(t)\right)$ in a distributed manner, and uses it to adjust the size of its contention window (see Table 3.2 for a formal description of this scheme in the form of an algorithm) in order to achieve an optimal channel access probability $P_{i}^{\tau}$, which leads to optimal throughput, as well as short-time fairness for each node. The variable maxtrans in Table 3.2 denotes the size of the observation window, which only depends on the number of transmission attempts. As ntrans increases, $\widehat{n}_{i}$ approaches $\bar{n}_{i}$. In the next section, methodology of obtaining $\mathcal{C}_{i}\left(\mathbf{P}^{\tau}(t)\right)$, only by listening for consecutive idle slots in the shared transmission medium is explained.

\subsubsection{Conditional collision probability estimation}

In order to have an accurate estimation of the conditional collision probability we have used the same method as in [14]. Therefore, the conditional collision probability can be 
Table 3.2: Medium Access Control Design

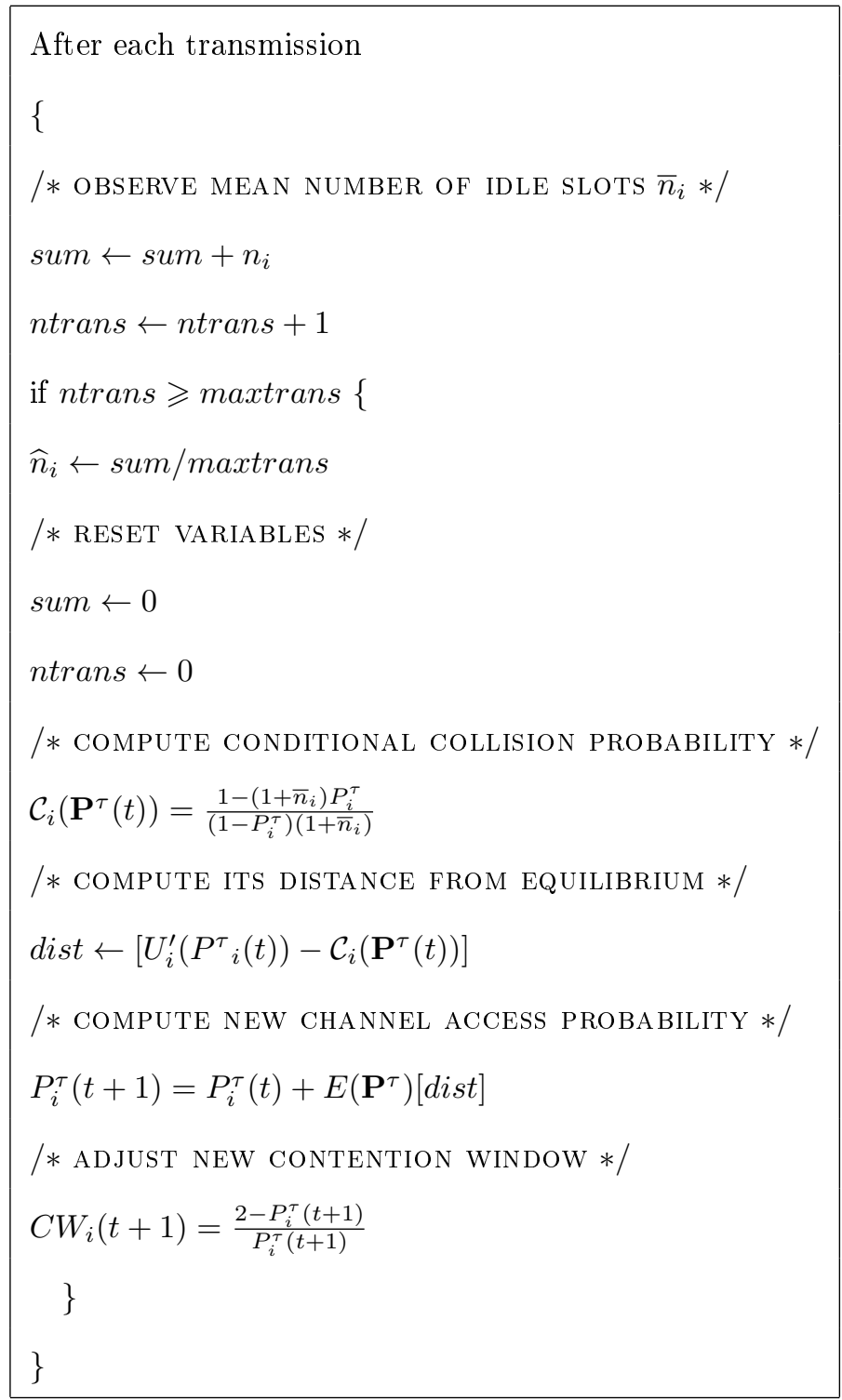

estimated via the observation of the wireless medium over several time slots. The parameters in our access method depend only on the protocol parameters that enable the game-theoretic model to provide a feedback scheme which does not need to differentiate between collided and corrupted frames. Thus, as aforesaid, we seek a channel access method that decouples the channel access probability from the conditional collision probability in order to provide us with the ability to discriminate between channel contention 
and occasional channel lack of reliability. Therefore, the conditional collision probability can be written using Eq. (3.2.5) and (3.2.13) as

$$
\mathcal{C}_{i}\left(\mathbf{P}^{\tau}\right)=1-\frac{P_{i}^{i d l e}\left(\mathbf{P}^{\tau}\right)}{1-P_{i}^{\tau}}
$$

Combining Eqs. 3.2.12 and 3.3.1 yields with good accuracy,

$$
\mathcal{C}_{i}\left(\mathbf{P}^{\tau}\right)=\frac{1-\left(1+\bar{n}_{i}\right) P_{i}^{\tau}}{\left(1-P_{i}^{\tau}\right)\left(1+\bar{n}_{i}\right)}
$$

which is determined through monitoring the consecutive idle time slots in the transmission medium only. The accuracy of conditional collision probability is proven by comparison with extensive simulations and literature [9].

\subsection{Summary}

A game-theoretic model for contention control has been developed and proposed a medium access method derived from CSMA/CA, in which each node estimates its state in a game and adjusts its persistence probability, or equivalently its contention window, according to its displacement from the Nash equilibrium. This results in harmonised but independently determined performance objectives for all competing nodes through the specification of per-node utility functions. As wireless nodes can estimate the conditional collision probability by observing consecutive idle slots between transmissions, it is possible to decouple contention control from handling failed transmissions. In Chapter 6, numerical results show, this model is capable of achieving much higher throughput than the standard IEEE 802.11 DCF with significantly better short-time fairness. Given that the network is in saturation mode, this also implies a low collision probability and channel access delay. The proposed protocol is highly dependent on the estimation of consecutive idle slot times. Therefore, a wrong choice of maxtrans, which defines the size of the observation window, 
can lead to a collapse of the game. Also, the step size must be chosen appropriately in order to converge to the desired point of operation with a reasonable convergence speed. This is an empirically arrived at compromise that balances convergence speed against protocol instability, which arises by choosing a larger step size for the update mechanism in the design. 


\section{CHAPTER 4}

\section{Game Theoretic DCF in Presence of}

\section{THE HidDEN TERMINALS}

As discussed in Chapter 2, the role of the MAC distributed coordination function (DCF) as the underpinning contention-based random channel access method has received much attention in the past decade ( $\mathrm{cf} \$ 2.2 .3$ to quantify its performance and improve its throughput and fairness. In literature (cf. \$2.2.2), it is shown that the performance of DCF is highly dependent on the number of hidden nodes in the network: These studies show that the presence of hidden nodes causes significant degradation in fairness and throughput due to the lack of time-synchronization between the hidden nodes. As DCF is expected to continue to play a prominent role in 5 th generation mobile communication technologies to address the issue of high bandwidth demand at base stations, eliminating its shortcomings remains a significant challenge.

This chapter seeks a channel access method that decouples the channel access probability $P_{i}^{\tau}$ from the conditional collision probability $P_{i}^{c}$, to minimise the effect of desynchronization amongst hidden nodes. Thus, in order to achieve good short-time fairness and higher throughput, a game-theoretic random access model based on CSMA/CA is proposed. This protocol provides an appropriate contention window size independently for each node in the network, resulting in an appropriate channel access probability for 
all nodes through local observation of consecutive idle slots between transmissions. The design of the game and utility function is reverse engineered through a system control point of view from the desired operation point using the DCF models in [39, 9]. A noncooperative, static, game theoretic approach to model the behaviour of contention-based wireless medium access was adopted. In noncooperative games, the players make rational, self-enforcing decisions considering only their individual payoffs; hence, the protocol operation is purely local. Due to the nature of the wireless channel, a static game in which all players make decisions without the knowledge of the remaining competing players' strategies was used(cf. \$2.4.1). In designing utility and payoff functions, a 802.11 DCF network with a single AP in the presence of hidden nodes is assumed. The concept of idle sense method was employed to design the utility and payoff functions, as proposed in [14], to derive the optimal number of consecutive idle time slots between transmissions based on the channel modulation scheme to avoid or minimise contention. A gradient play solution is used as a strategy update mechanism to dynamically control the contention window size by adjusting a current value of $P_{i}^{\tau}$ gradually towards its optimal value based on the number of idle slots observed between transmission attempts. Moreover, in Chapter 6 , numerical simulations of the proposed method in this chapter is demonstrated, including throughput, short-time fairness, collision rate, channel access delay, and delay distribution among the networks with different percentages of hidden terminals.

\subsection{Analysis of Channel Contention}

In this section, the effect of hidden terminal is introduced, the network topology that is used in the game will be discussed, and finally, an analytical model for DCF in the presence of hidden stations is presented. 


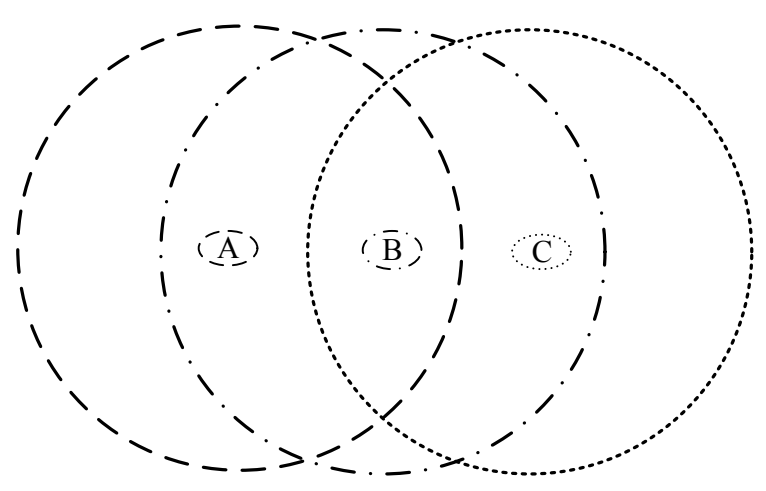

Figure 4.1: The classic hidden station

\subsubsection{Classic hidden station}

The classic hidden station scenario is shown in Figure 4.1, where both node $\mathrm{A}$ and $\mathrm{C}$ are in the carrier-sensing range of node B. However, node A and C cannot hear each other's transmission, and both may end up transmitting simultaneously. If one or both of those transmissions is destined for $\mathrm{B}$, then $\mathrm{B}$ will not be able to receive the packet successfully because of the data frame collision between $\mathrm{A}$ and $\mathrm{C}$. Therefore, node $\mathrm{C}$ is called a hidden terminal to A and vice versa (cf. Figure 4.1.

\subsubsection{The hidden terminal effect on the DCF (time-based point of view)}

The operation of the DCF channel access mechanism was discussed in $\$ 3.2 .1$ and will not be repeated here in the interest of brevity. The remarks here are confined to the scenario depicted in Figure 4.2. Taking $\mathrm{X}$ as the source for a particular transmission and AP as the destination, the backoff time measured in $b$ slots is a random transmission deferral time chosen uniformly from a distribution $[0, C W]$, where $C W$ is the contention window defined by the protocol. As shown in Figure 4.3, when a source transmits a data frame to the destination, the covered nodes sense the channel as busy, defer their transmission, 


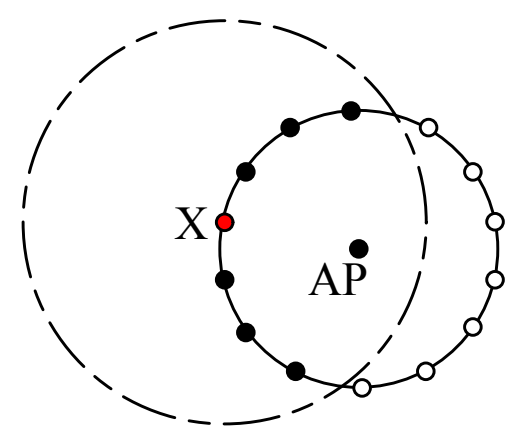

Figure 4.2: AP scenario in the presence of hidden nodes from the node $\mathrm{X}$ point of view: The dashed circle represents the carrier sense range of $\mathrm{X}$. The solid black circles represent nodes covered by both $\mathrm{X}$ and the AP, whereas the open circles represent hidden terminals for $\mathrm{X}$ but not the AP.

and freeze their backoff timers. A packet collision between the source and a covered node occurs only if the covered node transmits its frame before it senses the transmission from the source. The possible collision period between two nodes is called the vulnerable period $\left(\tau_{V}\right)$ [39, 46]. This period for a covered node in the basic access mechanism is the minimum period of time from the instant the source starts to transmit a data frame to the instant the covered node detects this ongoing transmission (defined as one slot time in IEEE 802.11 Figure 4.3). On the other hand, when the source transmits a data frame to the destination, the hidden nodes will sense the channel as idle until they receive an acknowledgement (ACK) frame from the destination. Any hidden node can transmit another frame to the destination during this period (a data frame together with a SIFS time duration). Hence, the vulnerable period for a hidden node in the basic access method equals the length of a data frame, as shown in Figure 4.3. As expected, the backoff period needs to be minimised to increase throughput, but maximised to reduce contention. From Figure 4.3 , it is evident that $\tau_{V}$ time interval depends on the backoff delay and the data frame duration.

Likewise, for a covered station in the RTS/CTS access mechanism, the basic access 


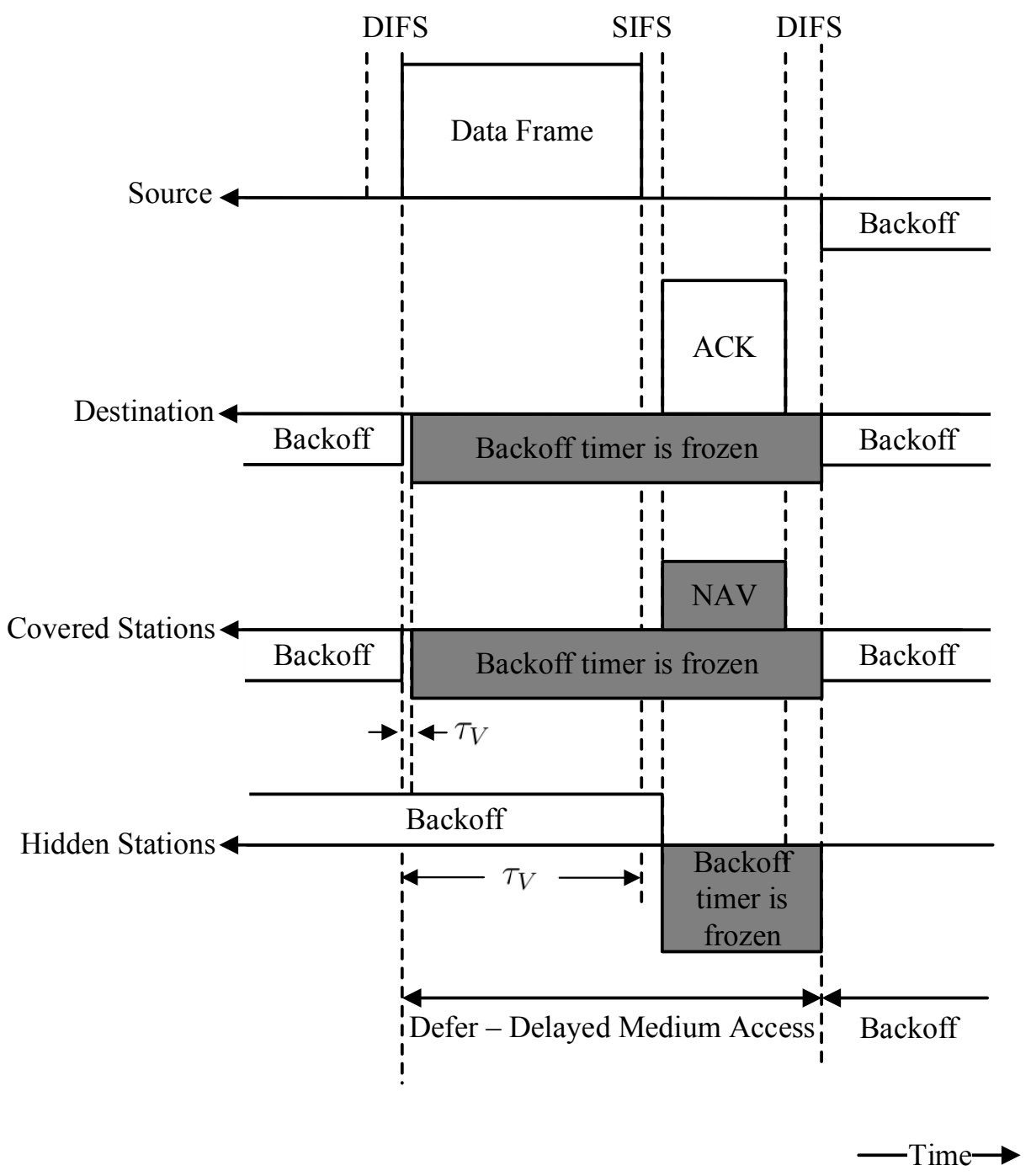

Figure 4.3: The vulnerable period $\left(\tau_{V}\right)$ : The basic access method. For details of all quantities, cf. [17]

mechanism $\tau_{V}$ time interval is also a slot time. In contrast, however, upon the transmission of a data frame to the destination, as incurred by the source, the hidden stations could sense the channel as being idle prior to receiving a CTS frame from the destination. Based on the RTS/CTS handshaking process, the destination will respond through only a CTS frame if it receives the RTS frame correctly and if it recognises the channel as idle. If the hidden station transmits another frame in the period of SIFS between the RTS and 


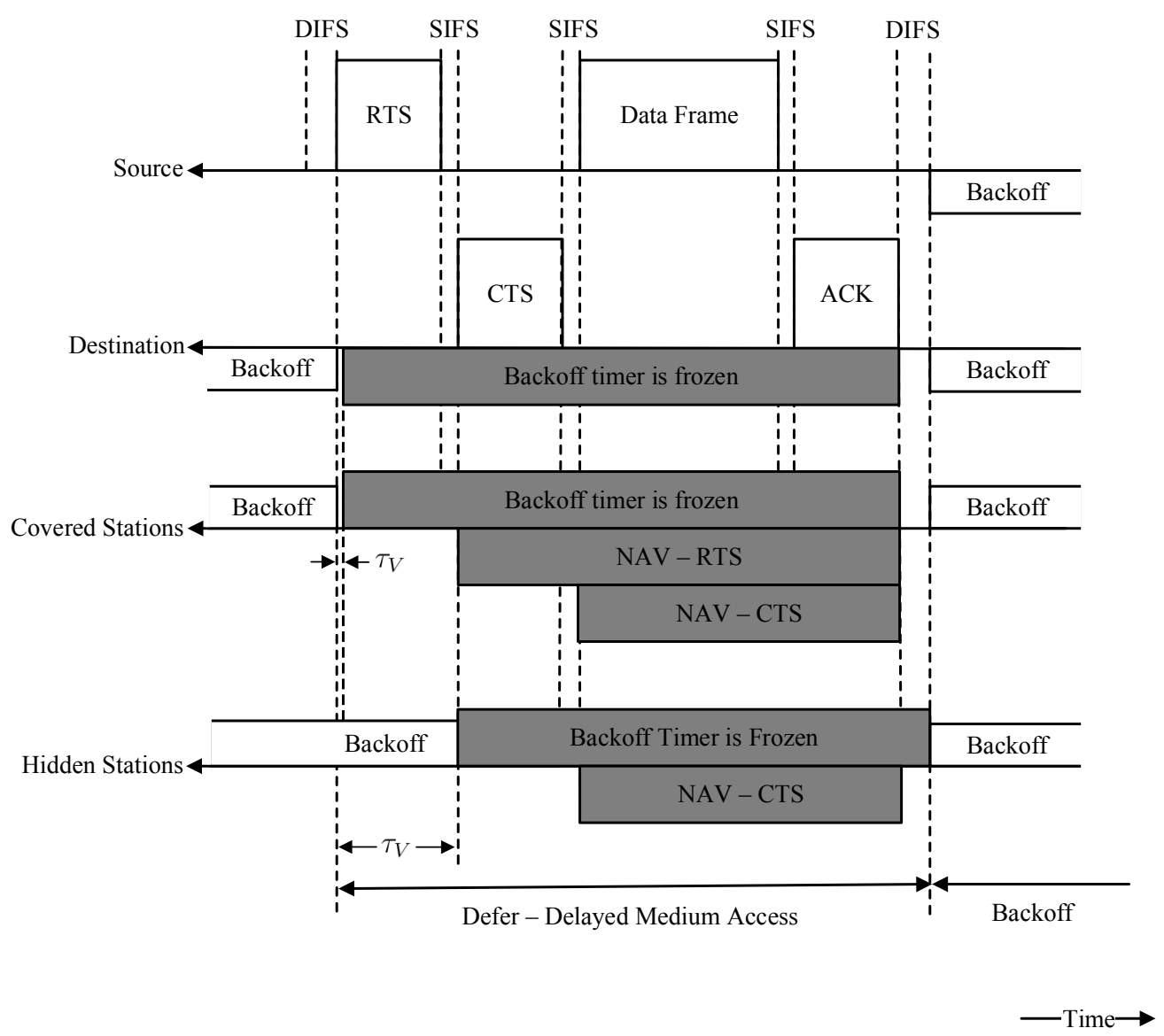

Figure 4.4: The vulnerable period $\left(\tau_{V}\right)$ : RTS/CTS access method. For details of all quantities, cf. [17]

CTS frame, the channel will be determined by the destination as busy, meaning it will not respond with a CST frame - even if the RTS frame is correctly received. Accordingly, in the case of a hidden station in the RTS/CTS access method, the $\tau_{V}$ is equal to the total an SIFS plus the RTS frame time period, as shown in Figure 4.4 .

Comparing the hidden station effect on the basic and RTS/CTS access mechanisms, the $\tau_{V}$ for the covered station in the RTS/CTS access method is same as that in the basic access mechanism. However, unlike the basic access method, the $\tau_{V}$ for hidden stations in the RTS/CTS access method is a fixed length period, a RTS frame plus a SIFS time, and does not depend on the length of the data frame from the source. So the RTS/CTS 
access mechanism reduces $\tau_{V}$ for the hidden stations. However, the RTS/CTS access method introduces an overhead, a RTS/CTS handshaking period, in every successful transmission which correspondingly decreases the network performance both in terms of network throughput and channel access delay.

\subsubsection{Analytical model of the DCF in the presence of the hidden stations}

The objective of the proposed method in this chapter is to minimise the effect of desynchronization amongst hidden nodes in both basic access and RTC/CTS mechanism, in order to minimise the collision probability in the $\tau_{V}$ time interval, without increasing $C W$ as aggressively as IEEE 802.11 DCF. First, an accurate relationship between $\tau_{V}$ and the conditional collision probability must be determined. Therefore, model in[39] is used as the underlying analytical model to compute $P_{i}^{c}$ and $P_{i}^{\tau}$.

In the case of the infrastructure mode in DCF [17], all of the stations are only able to transmit a frame to the AP; therefore, the network transmission can be categorised as upstream and downstream traffic: a frame sent from a station to the AP is known as upstream traffic, whereas a frame sent to the station from an AP is downstream traffic. Upon the transmission of a frame to the AP by the station, any station that is able to sense the source's transmission is referred to as a covered station. In contrast, any station that does not sense the transmission from the source but which is able to sense the transmission from the AP is referred to as a hidden station (cf. Figure 4.2). Accordingly, as it can be seen in Figure 4.2, in the case of the downstream traffic, there is no hidden station as all stations are able to sense the AP transmissions. Nonetheless, not all of the stations have the ability to sense transmissions in the upstream traffic, meaning, in this situation, the hidden station problem remains apparent.

In [39], the conditional collision probability of above scenario is evaluated using the two- 
dimensional Markov chain model. Based on chain regularities in [39], it is shown that the stationary probability $P_{i}^{\tau}$ that a covered station will, in a randomly selected time slot in the saturation conditions, transmit and then collide with the frame transmitted by the source, can be represented as follows[39]:

$$
\begin{aligned}
b_{0,0} & =\frac{2\left(1-P_{i}^{c}\right)\left(1-2 P_{i}^{c}\right)}{2\left(1-P_{i}^{c}\right)\left(1-2 P_{i}^{c}\right)+\left(1-2 P_{i}^{c}\right)\left(1-\left(P_{i}^{c}\right)^{m+1}\right)+W_{\min }\left(1-P_{i}^{c}\right)\left[1-\left(2 P_{i}^{c}\right)^{m+1}\right]}, \\
P_{i}^{\tau} & =\sum_{i=0}^{m} b_{i, 0}=\frac{1-\left(P_{i}^{c}\right)^{m+1}}{1-P_{i}^{c}} \cdot b_{0,0}
\end{aligned}
$$

where $m$ is the backoff stage $\left(C W_{j}=2^{j} C W, j \in(0, m)\right)$. The stationary probability $P_{i}^{\tau_{h}}$ that a hidden station will transmit during the $\tau_{V}$ period and collide with the frame transmitted by the source can be represented as [39]:

$$
\begin{gathered}
P_{i}^{\tau_{h}}=\sum_{i=0}^{m} \sum_{k=0}^{\tau_{V}} b_{i, k}= \\
\left\{\begin{array}{cc}
\left(\left(\tau_{V}+1\right) \cdot\left(\frac{1-P_{i}^{c^{m+1}}}{1-P_{i}^{c}}\right)-\left(\frac{\tau_{V}\left(\tau_{V}+1\right)}{2 C W_{\min }}\right) \cdot\left(\frac{1-\left(P_{i}^{c} / 2\right)^{m+1}}{1-\left(P_{i}^{c} / 2\right)}\right)\right) \cdot b_{0,0}, & \tau_{V}<C W_{\min } \\
\left(\frac{1}{2} \cdot\left(\frac{1-P_{i}^{c}}{1-P_{i}^{c}}\right)+\frac{C W_{\min }}{2} \cdot\left(\frac{1-\left(2 P_{i}^{c}\right)^{R}}{1-\left(2 P_{i}^{c}\right)}\right)+\left(\tau_{V}+1\right) \cdot\left(\frac{P_{i}^{c^{R}}-P_{i}^{c^{m+1}}}{1-P_{i}^{c}}\right)-\right. & \\
\left.\left(\frac{\tau_{V}\left(\tau_{V}+1\right)}{2 C W_{\min }}\right) \cdot\left(\frac{\left(P_{i}^{c} / 2\right)^{R}-\left(P_{i}^{c} / 2\right)^{m+1}}{1-\left(P_{i}^{c} / 2\right)}\right)\right) \cdot b_{0,0}, & C W_{R-1}<\tau_{V}<C W_{R} \\
1, & \tau_{V}>C W_{\max }
\end{array}\right.
\end{gathered}
$$

Where the $\tau_{V}$ duration is measured in the units of backoff slots. $R$ is the minimum backoff stage at which the contention window size is greater than $\tau_{V}(1 \leqslant R \leqslant m)^{1}$,

\footnotetext{
${ }^{1}$ Please Note that in $802.11 / \mathrm{b} \tau_{V}<C W_{\min }$
} 


\subsection{Formulation of Game Theoretic Random Access Model}

Consider a set of wireless network nodes $N$ with DCF as the contention protocol. Associated with each wireless node $i \in N$ is its channel access probability $P_{i}^{\tau}(t)$ and a certain contention measure observed by each node through the backoff mechanism. As stated in the description of DCF, each node in the network can observe its own channel access probability $P_{i}^{\tau}(t)$ and its conditional collision probability (contention measure) $P_{i}^{c}(t)$, but not those of other nodes. It can then adjust $P_{i}^{\tau}(t)$ based on a system control point of view, written as:

$$
P_{i}^{\tau}(t+1)=\mathcal{F}_{i}\left(P_{i}^{\tau}(t), P_{i}^{c}(t)\right)
$$

where time is slotted in units of a slot duration. The conditional collision probability depends on the channel access probability (i.e., the state of the broadcast medium), represented through a vector $\mathbf{P}^{\tau}(t)=\left(P_{i}^{\tau}(t), i \in N\right)$ :

$$
P_{i}^{c}(t)=\mathcal{C}_{i}\left(\mathbf{P}^{\tau}(t)\right)
$$

Here, $\mathcal{F}_{i}$ models the contention resolution algorithm and $\mathcal{C}_{i}$ models a mechanism, which updates the conditional collision probability in DCF. We assume that Equation 4.2.1 and 4.2.2 have an equilibrium $\left(\mathbf{P}^{\tau}, \mathbf{P}^{\mathbf{c}}\right)$. The fixed point of Equation 4.2.1 defines an implicit relation between the equilibrium $P_{i}^{\tau}(t)$ and $P_{i}^{c}(t)$. Then, by the implicit function theorem [82], there exists a unique, continuously differentiable function $F_{i}$, such that,

$$
P_{i}^{c}=F_{i}\left(P_{i}^{\tau}\right)
$$

Under the assumption of a Poisson arrival process, in DCF the conditional collision probability in the presence of hidden nodes is defined as [39], 


$$
\mathcal{C}_{i}\left(\mathbf{P}^{\tau}\right)=1-\left(1-P_{i}^{\tau}\right)^{n_{c}-1}\left(1-P_{i}^{\tau_{h}}\right)^{n_{h}}, \forall i \in N
$$

where $n_{c}$ is number of the covered nodes including the transmitting node, and $n_{h}$ is the number of the hidden nodes. The total number of contending nodes in the network $N$ is $N=n_{c}+n_{h}$ and $P_{i}^{\tau_{h}}$ will be defined in Equation 4.2.6.

The $C W$ in DCF is formulated in terms of the node channel access probability, $P_{i}^{\tau}$. When, $m=0$ (i.e., no exponential backoff) is considered, the $C W$ easily maps to a backoff mechanism by the following correspondence between $P_{i}^{\tau}$ and a constant contention window, that holds in saturation mode [9, 39] (the saturation mode implies that every node has a packet ready to transmit at all times) and it is independent of $P_{i}^{c}$ (cf. Equation 4.1.1):

$$
P_{i}^{\tau}=\frac{2}{C W+1}
$$

Moreover, as discussed above: no exponential backoff is considered, so based on Equation 4.1.1 and 4.1.2, $P_{i}^{\tau_{h}}$ can be written as a function of $P_{i}^{\tau}$ as follows:

$$
P_{i}^{\tau_{h}}=\left(\tau_{V}+1-\frac{\tau_{V}\left(\tau_{V}+1\right)}{2 C W_{\min }}\right) P_{i}^{\tau},
$$

A slowly varying contention window in the proposed utility function design is considered (theoretically, it is treated as a constant) in order to preserve the independence of $P_{i}^{\tau}$ from $P_{i}^{c}$. As will become clear later, such a choice of a "constant" contention window in the protocol plays a crucial role in guaranteeing the uniqueness of the Nash equilibrium, enabling the correct design of a payoff function and, more importantly, decoupling the $P_{i}^{\tau}$ from $P_{i}^{c}$. Therefore, the utility function of each node $i$ is defined as the following: 


$$
U_{i}\left(P_{i}^{\tau}\right)=\int_{v_{i}}^{P_{i}^{\tau}} F_{i}\left(P_{i}^{\tau}\right) d P_{i}^{\tau} .
$$

Based on the lack of dependency of $P_{i}^{\tau}$ on $P_{i}^{c}$ embodied in Equation 4.2.5 and the utility function 4.2.7, a random access game $G$ is defined as a triple $G=\left\{N,\left(S_{i}\right)_{i \in N},\left(u_{i}\right)_{i \in N}\right\}$, where $N$ is the set of players (nodes in the network). Player $i \in N$, adopts strategy $S_{i}=\left\{P_{i}^{\tau} \mid P_{i}^{\tau} \in\left[v_{i}, P_{i}^{\tau}\right]\right\}$ with $0<v_{i} \leqslant P_{i}^{\tau}<1$, and a payoff function $u_{i}\left(\mathbf{P}^{\tau}\right)=U_{i}\left(P_{i}^{\tau}\right)-$ $P_{i}^{\tau} \mathcal{C}_{i}\left(\mathbf{P}^{\tau}\right)$, with a utility function $U_{i}\left(P_{i}^{\tau}\right)$ and price function $P_{i}^{\tau} \mathcal{C}_{i}\left(\mathbf{P}^{\tau}\right)$. Note that the throughput and fairness of node $i$ depend on $P_{i}^{\tau}$ (as throughput is proportional to $P_{i}^{\tau}$ in the absence of collisions). $P_{i}^{\tau} \mathcal{C}_{i}\left(\mathbf{P}^{\tau}\right)$ represents the contention price for node $i$, for a given $P_{i}^{\tau}$. Therefore, potentially, each node can calculate precisely the cost of its action based on $P_{i}^{\tau}$, and with the knowledge that $P_{i}^{\tau}$ and $\mathcal{C}_{i}\left(\mathbf{P}^{\tau}\right)$ are independent of each other, has the ability to maximise its payoff function regardless of having direct knowledge of any other player's action at any specific time.

All the assumptions that will be used in the proposed game are same as the assumptions in last chapter. Please see $\$ 3.2 .2$.

\subsubsection{Nash Equilibrium}

The objective of the noncooperative game is to find the equilibrium solution for a wireless network with self-interested nodes in a distributed manner. A well-known solution for noncooperative games is the Nash equilibrium [13], which jointly maximizes all the players' utilities. Based on the game defined in Section 4.2, the Nash equilibrium satisfies the following condition [13]:

$$
u_{i}\left(P_{i}^{\tau *}, \mathbf{P}_{-i}^{\tau *}\right) \geqslant u_{i}\left(P_{i}^{\tau}, \mathbf{P}_{-i}^{\tau *}\right), \forall i \in N, \forall P_{i}^{\tau} \in S_{i},
$$

where $u_{i}($.$) is the payoff function of player i$, and $\mathbf{P}^{\tau *}{ }_{i}$ is a Nash equilibrium strategy vector for all the players except player $i\left(\mathbf{P}_{-i}^{\tau} \equiv\left(P_{1}^{\tau}, \ldots, P_{i-1}^{\tau}, P_{i+1}^{\tau}, \ldots, P_{N}^{\tau}\right)\right)$. Equation 4.2.8 
states that each player $i$, in choosing $P_{i}^{\tau *}$ is playing its best response to all the other players' strategy choices. A Nash equilibrium $\mathbf{P}^{\tau *}$ is a nontrivial equilibrium if $P_{i}^{\tau *}$ satisfies the following condition [13]:

$$
\frac{\partial u_{i}\left(P_{i}^{\tau *}, \mathbf{P}_{-i}^{\tau *}\right)}{\partial P_{i}^{\tau}}=0, \forall i \in N
$$

Note that Equation 4.2.9 does not guarantee that the equilibrium is unique and can admit solutions where the Nash equilibrium, $P_{i}^{\tau *}$ can take values at the boundaries of the strategy space, resulting in poor performance in terms of throughput and channel access delay (cf. assumption 2 in Section 4.2). However, if it satisfies Equation 4.2.10, then the equilibrium $P_{i}^{\tau *}$ is guaranteed [13] to be a unique nontrivial equilibrium:

$$
\frac{\partial U_{i}\left(P_{i}^{\tau *}\right)}{\partial P_{i}^{\tau}}=\mathcal{C}_{i}\left(\mathbf{P}^{\tau *}\right), \forall i \in N
$$

In order to meet the condition 4.2.10, the utility function $U_{i}\left(P_{i}^{\tau}\right)$ must be continuously differentiable and strictly concave. Since Equation 4.2.7 is integral and since $P_{i}^{c}=F_{i}\left(P_{i}^{\tau}\right)$ and $P_{i}^{c} \geqslant 0, F_{i}\left(P_{i}^{\tau}\right)$ is a continuous decreasing function (the larger the channel access probability, the smaller the conditional collision probability), it follows that $U_{i}\left(P_{i}^{\tau}\right)$ is a non-decreasing function: This implies that $U_{i}\left(P_{i}^{\tau}\right)$ is strictly concave. Hence, $u_{i}\left(\mathbf{P}^{\tau}\right)$ is continuous and concave in $P_{i}^{\tau}$, and there exists a nontrivial Nash equilibrium for game $G$ [13].

\subsubsection{Utility function design}

The utility function is formulated for each node in the network so as to ensure the existence of a unique nontrivial Nash equilibrium for each node. Based on the idle sense method [14], DCF from the desired operation point is reverse engineered. Consider a node attempting to transmit in a given time slot. the collision probability $P_{i}^{c}$ is denoted as the probability that two nodes in the network have the same value of $b$ and they transmit and collide at 
the same instant, or at least one hidden node transmits in the vulnerable period. Now let $P_{t r}$ be the probability that there is at least one transmission in the considered time slot:

$$
P_{t r}\left(\mathbf{P}^{\tau}\right)=1-\left(1-P_{i}^{\tau}\right)^{N}
$$

Thus, it is straightforward to express the probability of a successful transmission in a given slot, $P_{s}$, if $N$ nodes contend for the channel: Such an event requires a transmission attempt by a single node and the absence of all the others, including hidden and covered nodes:

$$
P_{s}\left(\mathbf{P}^{\tau}\right)=\frac{N P_{i}^{\tau}\left(1-P_{i}^{\tau}\right)^{n_{c}-1}\left(1-P_{i}^{\tau_{h}}\right)^{n_{h}}}{P_{t r}\left(\mathbf{P}_{\mathbf{i}}^{\tau}\right)} \cdot 2
$$

The collision probability in a slot can be expressed similarly:

$$
\mathcal{C}_{i}\left(\mathbf{P}^{\tau}\right)=1-P_{s}\left(\mathbf{P}^{\tau}\right) P_{t r}\left(\mathbf{P}^{\tau}\right)-P_{i}^{\text {idle }}\left(\mathbf{P}^{\tau}\right)
$$

where $P_{i}^{\text {idle }}$ is the probability of an idle slot and based on Equation 4.2.12 and 4.2.13. $P_{i}^{\text {idle }}$ can be written as:

$$
P_{i}^{\text {idle }}\left(\mathbf{P}^{\tau}\right)=\left(1-N P_{i}^{\tau}\right)\left(1-P_{i}^{\tau}\right)^{n_{c}-1}\left(1-P_{i}^{\tau_{h}}\right)^{n_{h}} .
$$

In [14, a medium access method is proposed that uses the mean number of consecutive idle slots between two transmission attempts. The mean number of consecutive idle slots between two transmission attempts (successful transmission, collision or frame corruption) is denoted by $\bar{n}_{i}$. This can be written as a function of $P_{i}^{\text {idle }}$ as following:

$$
\bar{n}_{i}=\frac{P_{i}^{\mathrm{idle}}\left(\mathbf{P}^{\tau}\right)}{1-P_{i}^{\mathrm{idle}}\left(\mathbf{P}^{\tau}\right)} .
$$

\footnotetext{
${ }^{2}$ Please note that when $N$ is large, the optimal channel access probability that maximises the channel utilization is very small (according to $802.11 / \mathrm{b} C W_{\min }=32$ and $C W_{\max }=1024$, see Equation 4.2.5.
} 
To derive the utility function the throughput is optimized by minimizing collision overhead and the time spent in idle slots. The throughput is expressed as a function of $P_{i}^{\tau}$ (cf. Equation 4.2.5) using [9, 39], such as what follows:

$$
S\left(P_{i}^{\tau}\right)=\frac{P_{s} P_{t r} L}{\left(1-P_{t r}\right) T_{\text {slot }}+P_{t r} P_{s} T_{s}+P_{t r}\left(1-P_{s}\right) T_{c}}
$$

In this expression, $T_{\text {slot }}$ is a slot duration that is a fixed protocol parameter. $T_{c}$ and $T_{s}$ are the average collision and transmission durations, respectively, and $L$ is the packet size. The average busy time after a collision by two mutually hidden nodes is denoted as $T_{c_{(\text {hidden })}}$, and the average busy time after a collision by two mutually covered stations is denoted as $T_{c_{\text {(covered) }}}$. Let $T_{\text {int }}$ denote the time interval between the start times of two colliding transmissions [39]. Denoting $\vartheta=P H Y_{\text {(header) }} / b r+M A C_{(\text {header })} / v, \widehat{\sigma}$ being the propagation delay and $A C K_{\text {(timeout) }}=S I F S+A C K / v+D I F S$, for the basic access mechanism we have:

$$
\begin{aligned}
& T_{s}=\vartheta+L+\widehat{\sigma}+S I F S+A C K+\widehat{\sigma}+D I F S . \\
& T_{c_{(\text {covered })}}=T_{i n t_{(\text {covered })}}+\vartheta+L+\widehat{\sigma}+A C K_{\text {(timeout) }} . \\
& T_{i n t_{(\text {covered })}}=(1 / 2) \times T_{\text {slot }} . \\
& T_{c_{(\text {hidden })}}=T_{i n t_{(\text {hidden })}}+\vartheta+L+\widehat{\sigma}+A C K_{(\text {timeout })} . \\
& T_{i n t_{(\text {hidden })}}=(1 / 2) \times(\vartheta+L) . \\
& T_{c}=\left(n_{c} / N\right) \times T_{c_{(\text {covered }}}+\left(n_{h} / N\right) \times T_{c_{(\text {hidden })} .} .
\end{aligned}
$$


Also for RTS/CTS the following is true:

$$
\begin{aligned}
& T_{s}=R T S+\widehat{\sigma}+S I F S+C T S+\widehat{\sigma}+S I F S+\vartheta \\
& +L+\widehat{\sigma}+S I F S+A C K+\widehat{\sigma}+D I F S . \\
& T_{c_{\text {(covered) }}}=T_{\text {int }} \text { (covered) }_{\text {(imeout }}+ \\
& T_{\text {int }} \text { (covered) }=(1 / 2) \times T_{\text {slot }} . \\
& T_{c_{\text {(hidden) }}}=T_{\text {int }_{\text {(hidden) }}}+R T S+\widehat{\sigma}+C T S_{\text {Timeout }} . \\
& T_{\text {int }_{(\text {hidden })}}=(1 / 2) \times(R T S+\widehat{\sigma}) . \\
& T_{c}=\left(n_{c} / N\right) \times T_{c_{(\text {covered })}}+\left(n_{h} / N\right) \times T_{c_{(\text {hidden })}} .
\end{aligned}
$$

where $C T S_{\text {Timeout }}=S I F S+C T S+\left(2 \times T_{\text {slot }}\right)$.

The objective is to calculate the optimal value $P_{i}^{\text {idle* }}$, so the first derivative of the throughput in Equation 4.2.16 is set to zero, $\partial S\left(P_{i}^{\tau *}\right) / \partial P_{i}^{\tau}=0$, to obtain $\bar{n}_{i}^{\text {opt }}$ for a given number of competing and hidden nodes for values of $P_{i}^{\text {idle* }}$ in $[0,1]$, corresponding to a variant of 802.11 summarized in Table 3.1 and Equation 4.2.17 and 4.2.18.

This leads to $P_{i}^{\tau *}$. Equation 4.2.14 and 4.2.15 give the optimal number of idle slots between two transmission attempts $\bar{n}_{i}^{\text {opt }}$ and thus the optimal contention window size $C W_{\text {opt }}$. We summarize $\bar{n}_{i}^{\text {opt }}$ for variable numbers of hidden nodes as the lower triangular matrix $\Psi_{i}$ :

$$
\left(\begin{array}{ccccc}
\bar{n}_{i_{(2,0)}^{\mathrm{opt}}}^{\mathrm{op}} & & & & \\
\bar{n}_{i_{(3,0)}^{\mathrm{opt}}} & \bar{n}_{i_{(3,1)}^{\mathrm{opt}}} & & 0 & \\
\vdots & \vdots & \ddots & & \\
\bar{n}_{i_{(k-1,0)}^{\mathrm{opt}}}^{\mathrm{ot}} & \bar{n}_{i_{(k-1,1)}^{\mathrm{opt}}} & \cdots & \bar{n}_{i_{(k-1, j)}^{\mathrm{opt}}} & \\
\bar{n}_{i_{(k, 0)}^{\mathrm{opt}}} & \bar{n}_{i_{(k, 1)}^{\mathrm{opt}}}^{\mathrm{opt}} & \bar{n}_{i_{(k, 2)}}^{\mathrm{opt}} & \cdots & \bar{n}_{i_{(k, j)}}^{\mathrm{opt}}
\end{array}\right)
$$


where $k$ is the total number of active nodes in the network, and $j$ is the number of hidden nodes from each node's point of view. If there are a total of 5 nodes in the network, a node with no hidden terminals should choose $\Psi_{i}(5,0)$, and another node with one hidden node should choose $\Psi_{i}(5,1)$ as its optimal $\bar{n}_{i}^{\text {opt }}$ (cf. Figures 4.5 and 4.6 .

According to the above procedure, the variation of $\bar{n}_{i}^{\text {opt }}$ for the basic access mechanism is highly sensitive to the vulnerable period because the size of $\tau_{V}$ depends on the size of the data frame (cf. Figure 4.3). Therefore, when the total number of stations increases and the fraction of covered and hidden nodes changes, $\bar{n}_{i}^{\text {opt }}$ varies dramatically and does not converge to the same value across the network (cf. Figure 4.5). In contrast, in the RTS/CTS access mechanism, $\bar{n}_{i}^{\text {opt }}$ varies slowly because the size of $\tau_{V}$ is fixed to the length of the RTS frame (cf. Figure 4.4). However, $\bar{n}_{i}^{\text {opt }}$ still does not converge to the same value across the network (cf. Figure 4.6). In order to reach equilibrium, stations must mirror the fact that they observe differing numbers of consecutive idle slots (c.f. Equation 4.2.19) between two transmission attempts, based on their associated number of hidden stations. Optimal contention window sizes $C W_{\text {opt }}$ for given $\bar{n}_{i}^{\text {opt }}$ are presented in Figures 4.7 and 4.8 .

Any deviation from $\bar{n}_{i}^{\text {opt }}$ will result in decreasing network performance in terms of throughput, fairness, and increased channel access delay. Therefore, without loss of generality, $P_{i}^{\text {idle* }}$ becomes the following:

$$
P_{i}^{\text {idle* }} \approx \mathcal{M}_{i}, \forall i \in N
$$

where $\mathcal{M}_{i}$ is defined based on the number of hidden nodes that node $i$ sees, as well as the total number of nodes in network:

$$
\mathcal{M}_{i}=\frac{\Psi_{i}(k, j)}{1+\Psi_{i}(k, j)}, \forall i \in N
$$




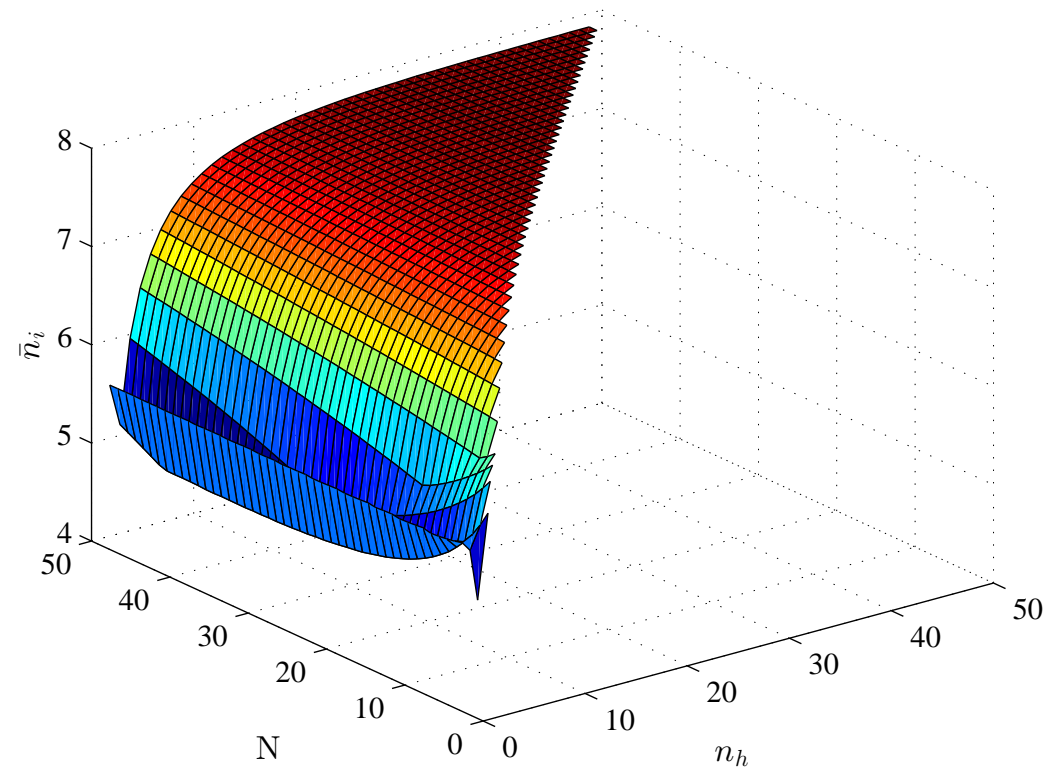

Figure 4.5: Number of nodes versus number of hidden nodes from each node point of view versus optimal number of idle slots $\bar{n}_{i}^{\text {opt }}$ for basic access mechanism based on the parameters in Table $3.1(802.11 / \mathrm{b})$.

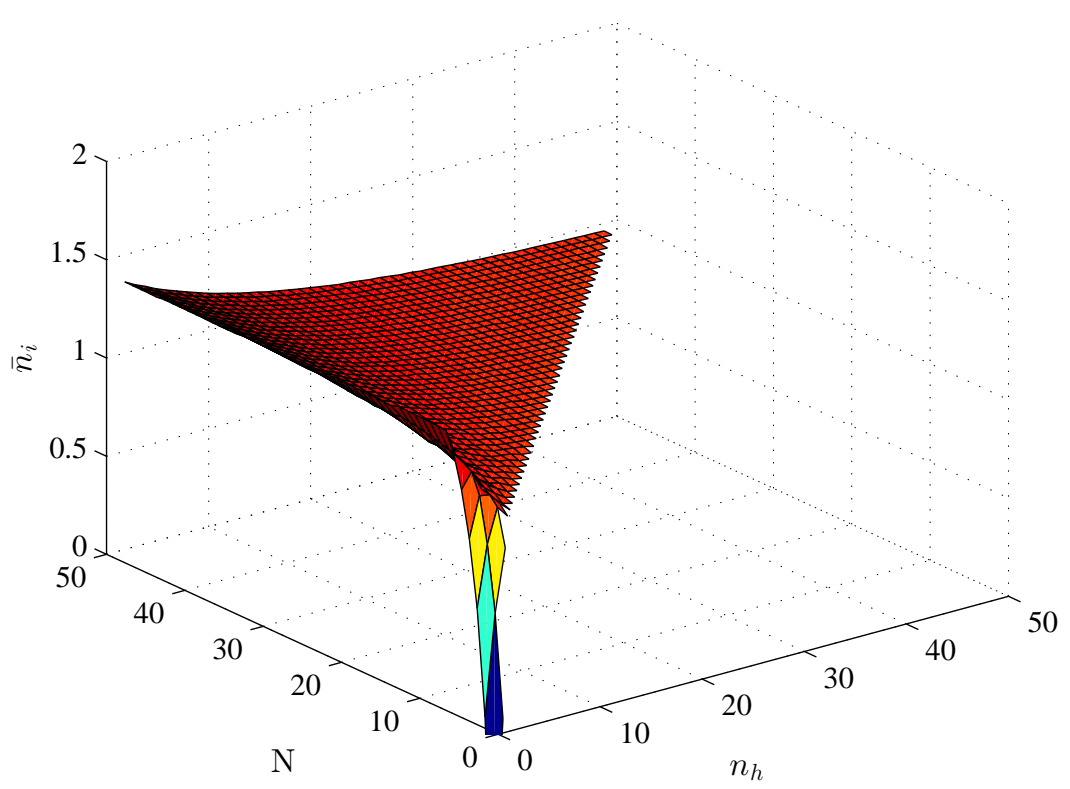

Figure 4.6: Number of nodes versus number of hidden nodes from each node point of view versus optimal number of idle slots $\bar{n}_{i}^{\text {opt }}$ for RTS/CTS access mechanism based on the parameters in Table 4.1 (802.11/b). 


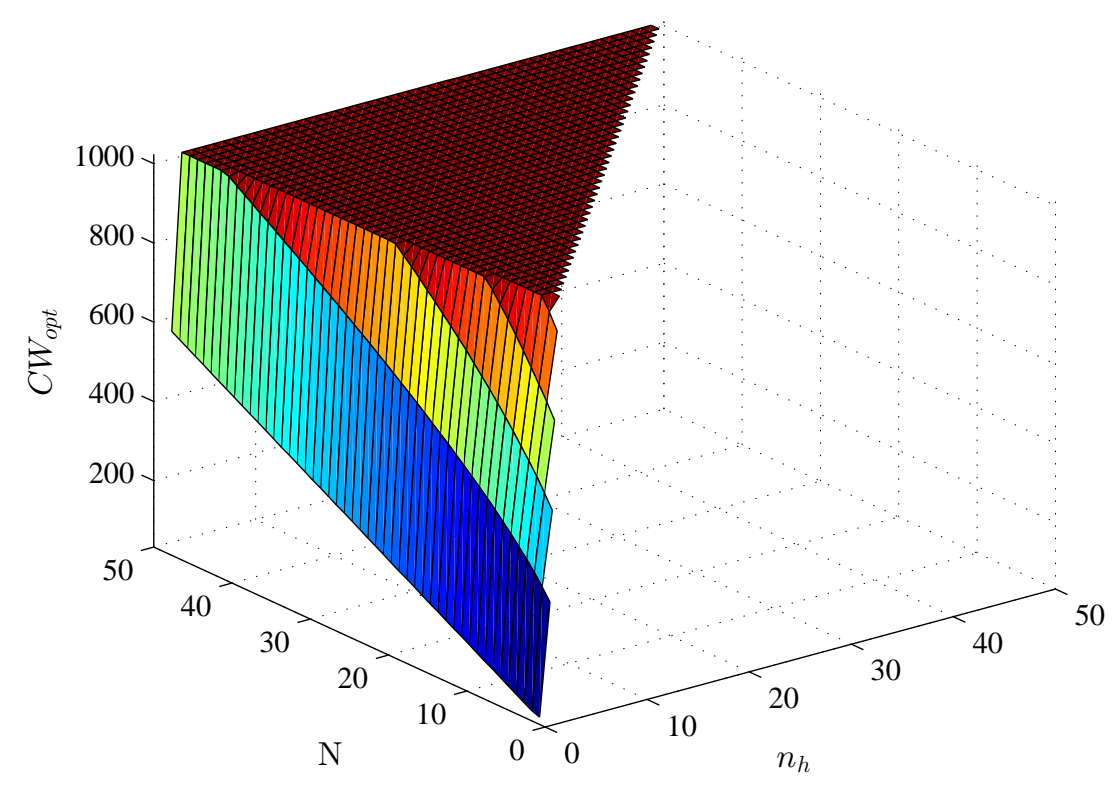

Figure 4.7: Number of nodes versus number of hidden nodes from each node point of view versus optimal values of contention window size $C W_{\text {opt }}$ for basic access mechanism based on the parameters in Table $3.1(802.11 / \mathrm{b})$.

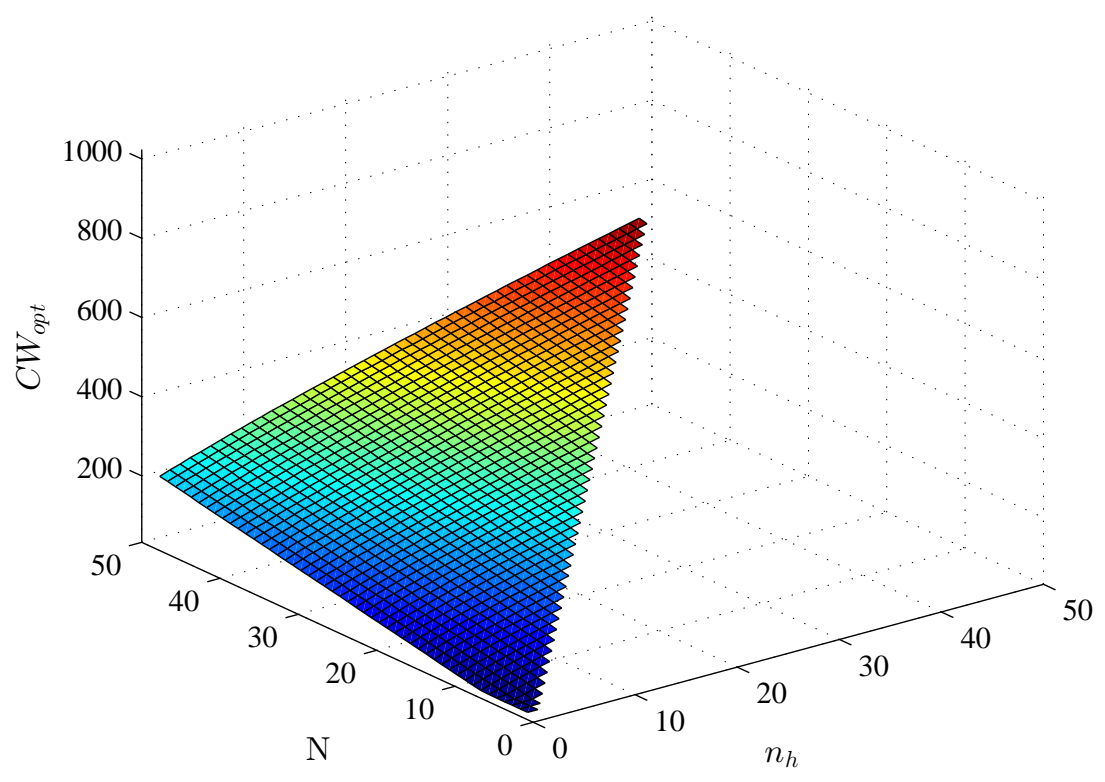

Figure 4.8: Number of nodes versus number of hidden nodes from each node point of view versus optimal values of contention window size $C W_{\text {opt }}$ for RTS/CTS access mechanism based on the parameters in Table $3.1(802.11 / \mathrm{b})$. 
In order to reverse engineer the utility function from the desired point of operation, it can be easily shown that,

$$
P_{i}^{\mathrm{idle}}\left(\mathbf{P}^{\tau}\right)=\left(1-N P_{i}^{\tau}\right)\left(1-U_{i}^{\prime}\left(P_{i}^{\tau}\right)\right), \forall i \in N
$$

where a prime denotes a derivative with respect to the function argument. According to Equation 4.2.20, the utility function at its maximum should be $P_{i}^{\text {idle }}\left(\mathbf{P}^{\tau}\right)=\mathcal{M}_{i}$. By applying Equation 4.2.7 we obtain the utility function. This function turns out not to be a strictly non-decreasing function; hence, it cannot guarantee the uniqueness of the Nash equilibrium. A convenient approximate choice that satisfies the uniqueness of the Nash equilibrium is the following:

$$
P_{i}^{\text {idle }}\left(\mathbf{P}^{\tau}\right)=\mathcal{M}_{i}\left(1+P_{i}^{\tau}\right)
$$

Note that when $N$ is large, the optimal channel access probability that maximizes the throughput is very small [39, 14]. Applying Equation 4.2.7, the utility function is derived as follows:

$$
U_{i}\left(P_{i}^{\tau}\right)=P_{i}^{\tau}\left(\frac{\mathcal{M}_{i}}{N}+1\right)+\frac{\ln \left(1-N P_{i}^{\tau}\right)\left(\mathcal{M}_{i}+N \mathcal{M}_{i}\right)}{N^{2}}
$$

Therefore, the payoff function now becomes this:

$$
\begin{array}{r}
u_{i}\left(\mathbf{P}^{\tau}\right)=P_{i}^{\tau}\left(\frac{\mathcal{M}_{i}}{N}+1\right)+\frac{\ln \left(1-N P_{i}^{\tau}\right)\left(\mathcal{M}_{i}+N \mathcal{M}_{i}\right)}{N^{2}} \\
-P_{i}^{\tau} \mathcal{C}_{i}\left(\mathbf{P}^{\tau}\right) .
\end{array}
$$


Table 4.1: Medium Access Control Design

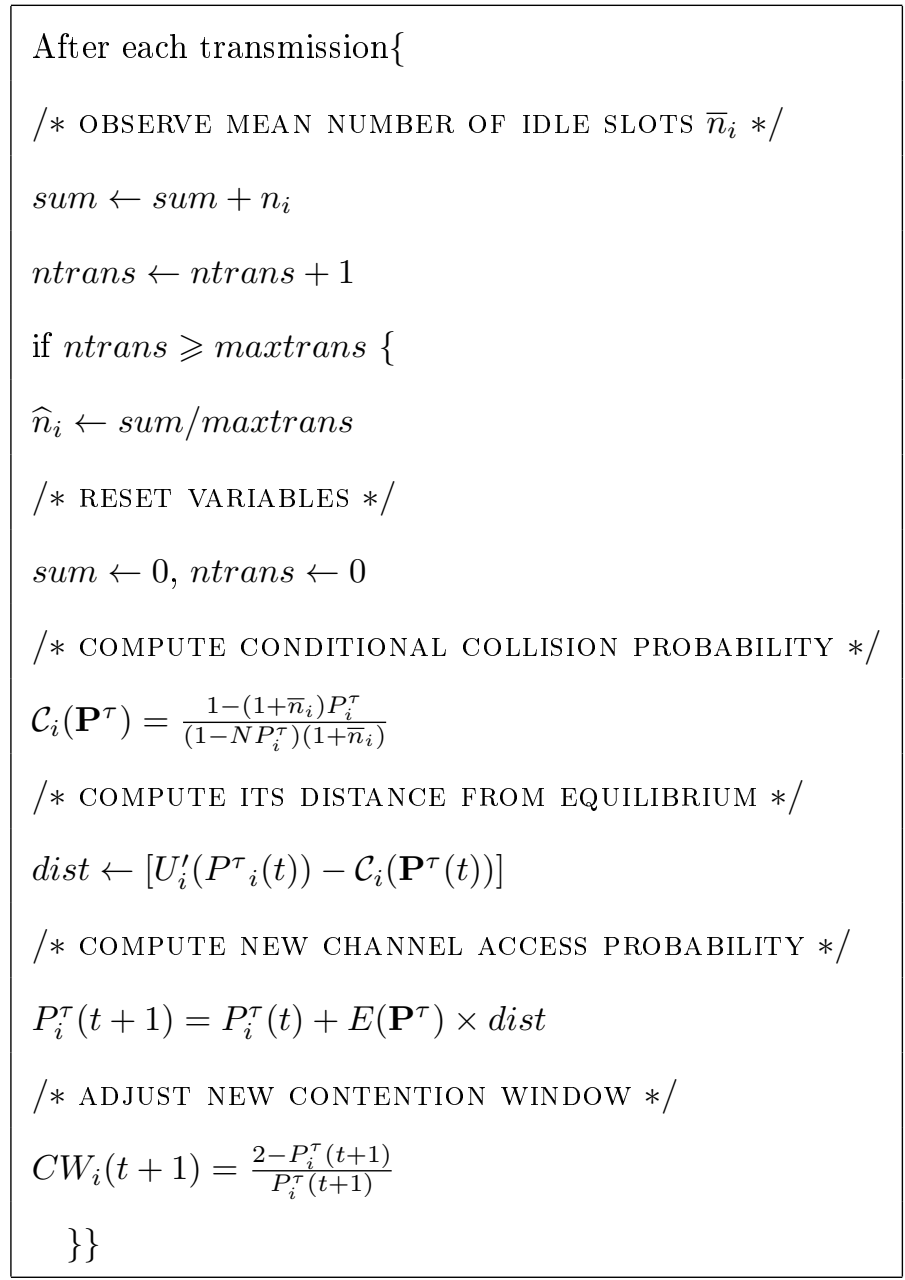

\subsubsection{Dynamics of random access game}

An investigation on how interacting players converge to a Nash equilibrium is the next step. Repeated play of the random access game is considered, and an update mechanism in which players repeatedly adjust strategies in response to observations of other player' actions is sought so as to achieve the Nash equilibrium. The simplest and most common strategy update mechanism is sorting the best response at each stage. This means that every node chooses the best response to the previous round of the game. Mathematically, 
at stage $t+1$, node $i$ chooses a channel access probability using the following mechanism:

$$
P_{i}^{\tau}(t+1)=\underset{P_{i}^{\tau} \in S_{i}}{\operatorname{argmax}}\left(U_{i}\left(P_{i}^{\tau}(t)\right)-P_{i}^{\tau}(t) \mathcal{C}_{i}\left(\mathbf{P}^{\tau}(t)\right) .\right.
$$

Evidently, if the above mechanism reaches to a steady state, then this state is a Nash equilibrium. However, there are no convergence results for noncooperative games using this mechanism (cf. \$2.4.2). Therefore, we adopt the gradient play [84] strategy update mechanism. Each node $i \in N$ updates its strategy according to,

$$
P_{i}^{\tau}(t+1)=P_{i}^{\tau}(t)+E\left(\mathbf{P}^{\tau}\right)\left[U_{i}^{\prime}\left(P_{i}^{\tau}(t)\right)-\mathcal{C}_{i}\left(\mathbf{P}^{\tau}(t)\right)\right]
$$

where $E\left(\mathbf{P}^{\tau}\right)>0$ is the update step size. At stage $t+1$, node $i \in N$ chooses a channel access probability derived from stage $t$. Then each node $i \in N$ updates its contention window $C W_{i}$ as follows:

$$
C W_{i}(t+1)=\frac{2-P_{i}^{\tau}(t+1)}{P_{i}^{\tau}(t+1)} .
$$

The gradient play admits a nice economic interpretation, if we interpret the conditional collision probability $\mathcal{C}_{i}\left(\mathbf{P}^{\tau}(t)\right)$ as contention price for node $i$. If the marginal utility $U_{i}^{\prime}\left(P_{i}^{\tau}(t)\right)$ is greater than the contention price, we increase the channel access probability. Conversely if the marginal utility is less than contention price, we decrease the access probability; then using Equation 4.2 .28 each node updates its strategy. The step size determines the convergence speed. In our game we choose a constant step size $E\left(\mathbf{P}^{\tau}\right)$ for all nodes. The step size empirically arrived at a compromise that balances convergence speed against protocol instability. Each node in the network might see different number of hidden and covered terminals (each node has different $\mathcal{M}_{i}$ ). Therefore, the payoff function is different for each node (cf. Equation 4.2.25). This means nodes in the network converge to the equilibrium in different number of transmissions. However, 
in comparison with single-cell networks in the previous chapter both proposed protocols have close overall convergence speed.

\subsection{Medium Access Control Design}

Using section 4.2, a medium access model is designed assuming that the AP can determine and periodically broadcast the number of active nodes. Then, instead of using a binary exponential backoff scheme, each station computes locally its displacement from equilibrium using $U_{i}^{\prime}\left(P^{\tau}{ }_{i}(t)\right)-\mathcal{C}_{i}\left(\mathbf{P}^{\tau}(t)\right)$ in a distributed manner and uses it to adjust the size of its contention window (see Table 4.1 for a formal description of this scheme in the form of an algorithm). The variable maxtrans in Table 4.1 denotes the size of the observation window, which only depends on the number of transmission attempts. As ntrans increases, $\widehat{n}_{i}$ approaches $\bar{n}_{i}$.

\subsubsection{Conditional collision probability estimation}

In order to estimate accurately the conditional collision probability, the same method as [14] has been used. Therefore, the conditional collision probability can be estimated via the observation of the wireless medium over several time slots and can be written using Equations 4.2.4 and 4.2.14 as the following:

$$
\mathcal{C}_{i}\left(\mathbf{P}^{\tau}\right)=1-\frac{P_{i}^{\text {idle }}\left(\mathbf{P}^{\tau}\right)}{1-N P_{i}^{\tau}}
$$

Combining Equation 4.2.15 and 4.3.1 yields with good accuracy:

$$
\mathcal{C}_{i}\left(\mathbf{P}^{\tau}\right)=\frac{1-\left(1+\bar{n}_{i}\right) P_{i}^{\tau}}{\left(1-N P_{i}^{\tau}\right)\left(1+\bar{n}_{i}\right)}
$$

This is determined through monitoring the consecutive idle time slots in the transmission medium and broadcasting the number of active nodes by the AP. The accuracy of 
conditional collision probability is proven by comparison with extensive simulations and literature [9, 39]. This estimation leads to extremely accurate results, especially when the number of stations in the network is fairly large (say $N \geqslant 10$ ).

\subsection{Summary}

This study has developed a game-theoretic model for contention control and proposes a medium access method derived from CSMA/CA, in which each node estimates its state in a game and adjusts its persistence probability or contention window according to its displacement from the Nash equilibrium. The novelty of the protocol is that, to the best of author's knowledge, it is the first such protocol to explicitly account for the effect of hidden nodes and mitigate for it purely through local observations in the context of game theory. The correct specification of per-node utility functions results in harmonized but independently determined performance objectives for all competing nodes. As wireless nodes can estimate the conditional collision probability by observing consecutive idle slots between transmissions, contention control can be decoupled from the handling of failed transmissions. As it will be shown in chapter 6 the proposed game-theoretic medium access control design exhibits low channel access delay and better throughput which ultimately results in good short-term fairness.

The proposed protocol is highly dependent on the estimation of consecutive idle slot times. Therefore, a wrong choice of maxtrans, which defines the size of the observation window, can lead to a collapse of the game. Also, the step size must be chosen appropriately in order to converge to the desired point of operation with a reasonable convergence speed. This is an empirically arrived at compromise that balances convergence speed against protocol instability that arises by choosing a larger step size for the update mechanism in this design. 


\section{CHAPTER 5}

\section{Simulation Methodology}

\subsection{Introduction}

$\mathrm{OMNeT}++$ is an object-oriented modular discrete event network simulation framework [85]. "OMNeT ++ itself is not a simulator of any specific system; however, it provides infrastructure and tools for writing simulations. It has a generic architecture; therefore, it can be used in modelling and simulation of any system where the discrete event approach is suitable and can be conveniently mapped into entities communicating by exchanging messages. One of the fundamental ingredients of OMNet ++ 's infrastructure is a component architecture for simulation models. Models are assembled from reusable components termed modules. Well-written modules are truly reusable, and can be combined in various ways like LEGO blocks" [85].

Numerical experiments have been conducted using $\mathrm{OMNeT}++$. The game-theoretic MAC protocols that were discussed in chapter 3 and 4 were implemented into the standard 802.11 DCF basic access method and RTS/CTS in order to evaluate their performance in comparison to the original access mechanisms.

This chapter describes briefly the $\mathrm{OMNeT}++$ simulation framework and introduces the design of the study's wireless network in the simulation environment. Moreover, it details the development work of a number of extensions that were added to the existing protocol 
stack in order to implement the game theoretic MAC protocols. The chapter presents the implementation of various simulation aspects, such as the network topology parameters and traffic patterns parameters. A number of validation studies are also presented. Finally, a number of performance metrics are defined to measure the channel throughput, channel access delay, and fairness of the MAC protocols.

\subsection{Simulation Environment}

A network simulator is an efficient and cost-effective way to measure how the network or protocol would behave in any given hypothetical conditions or scenarios. Most network simulators vendors provide source code for users in order to enable them to customize the simulator in a way to fulfil their specific simulation and analysis needs. "Discrete event simulations store a list of pending "events", and these events are processed in order, with some events triggering future events, such as the event of the departure of a packet from one node triggering the event of the arrival of that packet at a downstream node" [85]. Some network simulation problems, notably those relying on queuing theory, are well suited to Markov chain simulations; in that a list of future events is maintained and the simulation consists of transiting between different system "states" in a memory-less fashion. Markov chain simulation is typically faster but less accurate and flexible than detailed discrete event simulation. Therefore, to have accurate and detailed results for this study, a number of discrete-event network simulators were evaluated.

There are many discrete-event network simulators available for wireless networks [86]. Some of the well-known simulators include Network Simulator 2 (Ns-2) [87], Opnet Modeler[88], Jist/SWANS[89], QualNet (previously GloMoSim)[90] and OMNeT ++ [85]. These simulators run over the GNU/Linux, FreeBSD, Solaris, Mac OS, and Microsoft Windows operating systems. A brief evaluation of $\mathrm{Ns} 2$ in order to implement our MAC protocols revealed a programming model that used a combination of a $\mathrm{C}++$ for simulation 
OSI

\begin{tabular}{|c|}
\hline Application \\
\hline Presentation \\
\hline Session \\
\hline Transport \\
\hline Network \\
\hline Data link \\
\hline Physical \\
\hline
\end{tabular}

MiXiM

\begin{tabular}{|l|}
\hline appl \\
\hline N/A \\
\hline N/A \\
\hline N/A \\
\hline netwl \\
\hline mac \\
\hline phy \\
\hline
\end{tabular}

Figure 5.1: OSI model vs MiXiM model

models backed by a tcl scripting engine, resulting in complex simulation code and a timeconsuming programming task. Opnet was discounted as the kernel source code was not readily available, and this was required for the implementation of the MAC layer access to the physical layer to calculate the consecutive idle time slots (cf. $\$ 5.3 .2$ ). Jist/SWANS and GloMoSim are no longer under active development and simulated entities are buggy and incomplete, especially for implementing IEEE 802.11. Finally, for this study, OM$\mathrm{NeT}++$ was adopted as simulator for a number of reasons. The author was familiar with the $\mathrm{C}++$ language which is the programming language for modules in $\mathrm{OMNeT}++$. More importantly OMNeT ++ come with MiXiM! which has a detailed radio wave propagation and 802.11 MAC protocol by default. The availability of radio wave propagation model was especially important to determine the effects these had on time-synchronization among the nodes in both single-cell networks and centralised networks with hidden terminals in order to evaluate the proposed MAC protocol's performance (cf. Chapter 3

\footnotetext{
1 "MiXiM is an OMNeT ++ modeling framework created for mobile and fixed wireless networks (wireless sensor networks, body area networks, ad-hoc networks, vehicular networks, etc.). It offers detailed models of radio wave propagation, interference estimation, radio transceiver power consumption and wireless MAC protocols (e.g. 802.11, Zigbee, etc.)."[91]
} 
and (4). Moreover the simulations can be run under various user interfaces. Graphical, animated user interfaces are highly useful for demonstration and debugging purposes, and command-line user interfaces are best for batch execution [85]. These interfaces allow us to build a realistic simulation for the proposed MAC protocols, where particular aspects of protocol implementation can be isolated and individually tested. None of the above simulators have all these features together except Opnet, for which the kernel source code was not available. Additionally, the MiXiM Application Programming Interface (API) was well-documented and facilitated rapid prototyping and was easy to understand as the MiXiM is organised into a set of sub modules which corresponded roughly to the classical OSI model (cf. Figure 5.1).

\subsection{Simulation Configuration}

Credible simulation studies, which ultimately could produce correct and trustworthy simulation results, must be verified and validated in order to confirm a simulation model is implemented correctly with respect to its mathematical (conceptual) model [92, 93, 86]. IEEE 802.11 MAC protocol model has been implemented and configured for both access mechanisms (RTS/CTS \& basic access) to run as a baseline for comparison, verification and validation of simulator behaviour against the proposed game theoretic MAC protocols. According to [86]: "Valid and credible simulation studies must be repeatable, unbiased, rigorous and results must be statistically sound with respect to the mathematical models". This section introduces the network topology, network and node model, and the environmental parameters used to design the network simulations. The methods used to evaluate the validity of the baseline protocols are also detailed. The script code for the details of the simulation configuration, the "omnetpp.ini" file is given in Appendix C. 
Table 5.1: Generating random locations of nodes

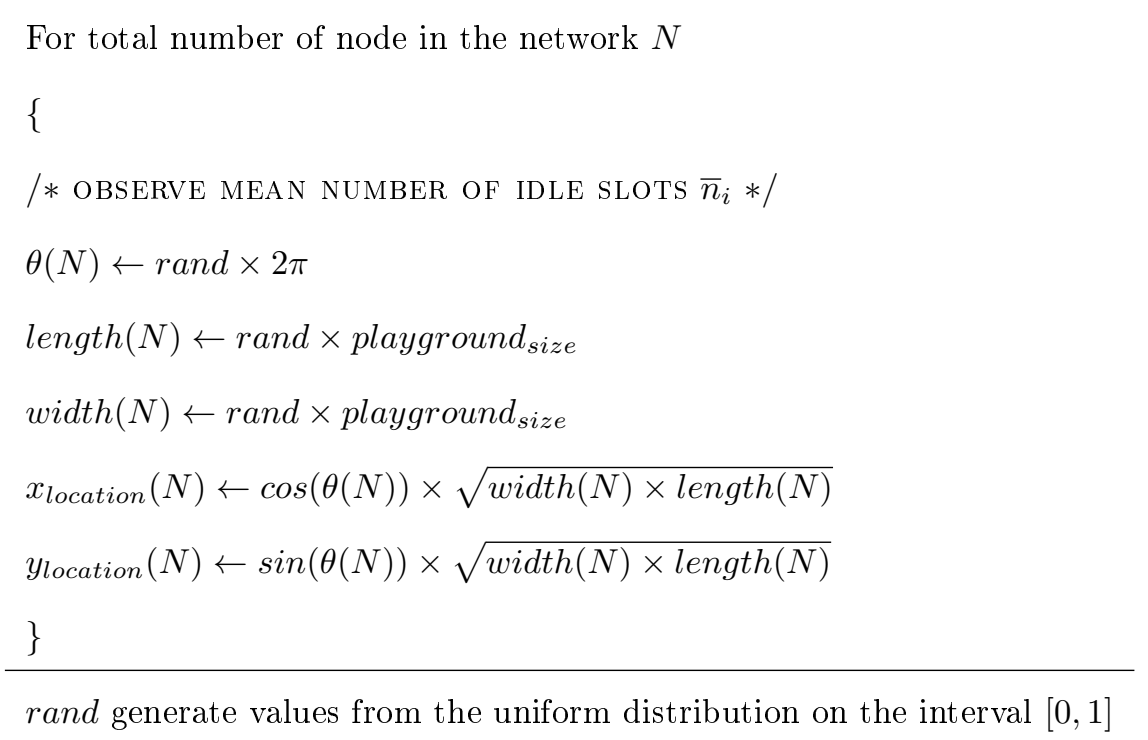

\subsubsection{Simulation topology}

The topology for the proposed protocol in Chapter 3 is ad hoc and assumes there are no hidden terminals in the network. Therefore, nodes were randomly located around the centre of the simulation area ("playground"), which is a square. The diagonal of the area was set as the maximum sensing range of the nodes to guarantee the absence of hidden terminals in the network. Specific and realistic simulator environment parameters, such as the phy layer, transmit power and phy sensitivity, etc. (cf. Appendix C) were used. The same parameter settings were re-used in the proposed MAC protocols for the sake of drawing meaningful comparisons. Moreover, simulations were run for different numbers of nodes and in Chapter 4 also with a different number of hidden nodes in the network. This is done to ensure that fair and representative performance comparisons are made. All these measures were taken to make the simulation fair, unbiased and rigorous. The size of the networks is varied from 5 active nodes up to 40 active nodes. Table 5.1 shows the formal algorithm description for generating the location of simulation nodes based on the assumptions of Chapter 3. Figure 5.2 shows one of the scenarios of an ad-hoc network 


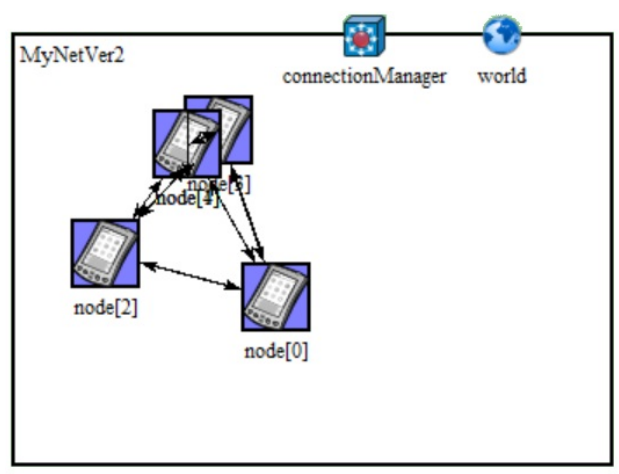

Figure 5.2: Ad hoc network topology with 5 nodes and no hidden terminal.

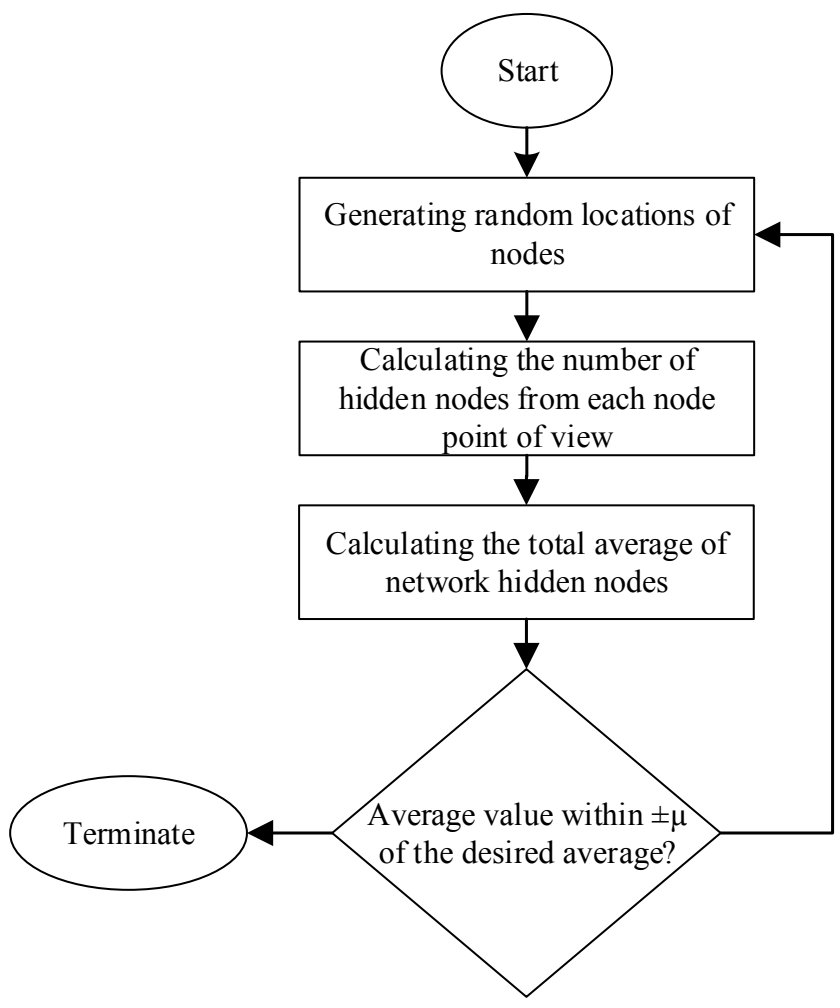

Figure 5.3: Calculation of network with specific hidden node percentage $(\mu)$.

topology on the OMNeT ++ graphical interface.

Chapter 4 assumes an infrastructure topology. In order to evaluate the protocol's performance for all possible scenarios, the simulation topology was designed based on the 


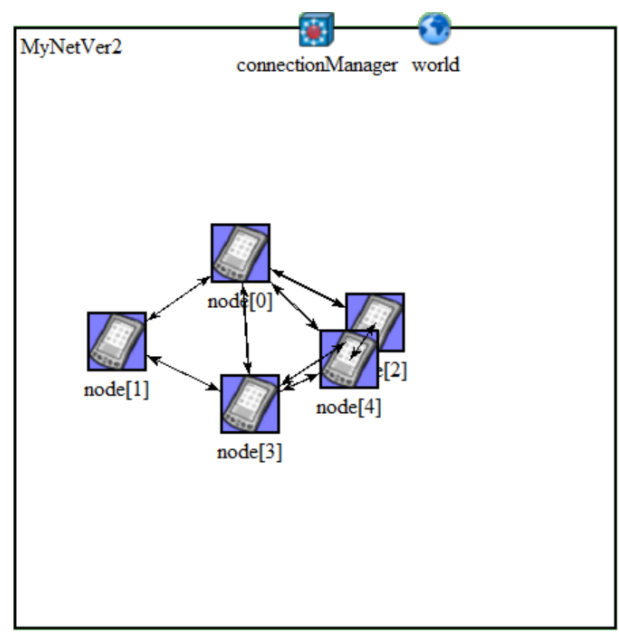

Table 5.2: The number of hidden and covered stations in scenario depicted in Figure 5.4 from each node's point of view:

\begin{tabular}{|c|c|c|}
\hline$N$ & Hidden & Covered \\
\hline node[0] & 0 & 4 \\
\hline node[1] & 2 & 2 \\
\hline node[2] & 1 & 3 \\
\hline node[3] & 0 & 4 \\
\hline node[4] & 1 & 3 \\
\hline Percentage & $20 \%$ & $80 \%$ \\
\hline
\end{tabular}

Figure 5.4: An example of centralised topology. A network with 5 nodes and $20 \%$ hidden terminal.

percentage of total hidden nodes in the network. Calculating the theoretical average number of hidden stations has to be done based on each station's point of view of the distribution of node locations around the AP. In this study, a uniform spatial distribution is assumed. However, any other distribution can be assumed. The problem is formalized as follows: $N$ stations are randomly (uniformly) located around the AP (cf. Table 5.1). For each station, it is determined how many of the remaining $N-1$ stations are hidden to that particular station. Then, if the average hidden nodes in the network are the desired percentage $(\mu)$ for the scenario, it will be updated to the omnetpp.ini file. Otherwise, the process will be repeated till the desired average for the scenario is reached (cf. Figure 5.3). For example, Figure 5.4 and Table 5.2 shows a $20 \%$ hidden nodes scenario in the infrastructure topology according to the assumptions discussed in Chapter 4.

\subsubsection{Network modelling}

"A MiXiM node model in OMNeT ++ consists of hierarchically nested sub modules that communicate by exchanging messages with each other" [91]. These sub modules are written in $\mathrm{C}++$, using the OMNeT ++ simulation class library and MiXiM's APIs. As men- 
tioned earlier, these sub-modules correspond roughly to the classical OSI model (Figure 5.1). Sub modules communicate with messages that contain arbitrary data, in addition to usual attributes, such as a time-stamp. They send messages via gates to their destination modules. Gates are the input and output interfaces of modules: messages are sent through output gates and arrive through input gates. An input gate and output gate are linked by a connection (Figure 5.5). All the sub modules have parameters ${ }^{2}$ that are assigned in the configuration file omnetpp.ini. These parameters are detailed in Appendix C.

Typical 802.11 networks in MiXiM are shown in Figures 5.2 and 5.4 . The connection manager module is responsible for providing the connections between the wireless MiXiM NICs (Network Interface Card). Parameters such as propagation delay, data rate, and bit error ratio are assigned to connections using the connection manager. Also, each node in the network consists of modules for its application, network, and MAC layers (cf. Figure $5.5)$.

A number of functions were developed for the different parts of the protocol stacks used in implementing the game theoretic MAC protocols. Based on the assumptions discussed in Chapters $3 \&$ 4, all nodes in the network are in saturation mode, which requires that all nodes have a packet ready to send. At the application layer based on the total number of the nodes in the network, each node generates its portion of the data traffic in such a way as the summation of all generated traffic streams brings network into saturation mode. Moreover, addressing the packets has been done completely randomly and based on the topology of the network. In a centralised topology, after authentication, the AP broadcasts the total number of active nodes to the network in order to enable the nodes to calculate their distance from equilibrium in a distributed manner (cf. Section 4.3). In the MAC layer, a number of functions were designed in which each node, by con-

\footnotetext{
2"Parameters can take string, numeric or boolean values, or can contain XML data trees. Numeric values include expressions using other parameters and calling $\mathrm{C}$ functions, random variables from different distributions, and values input interactively by the user". 911 Parameters are used to customize the sub modules behaviour, and to parametrize the model topology.
} 


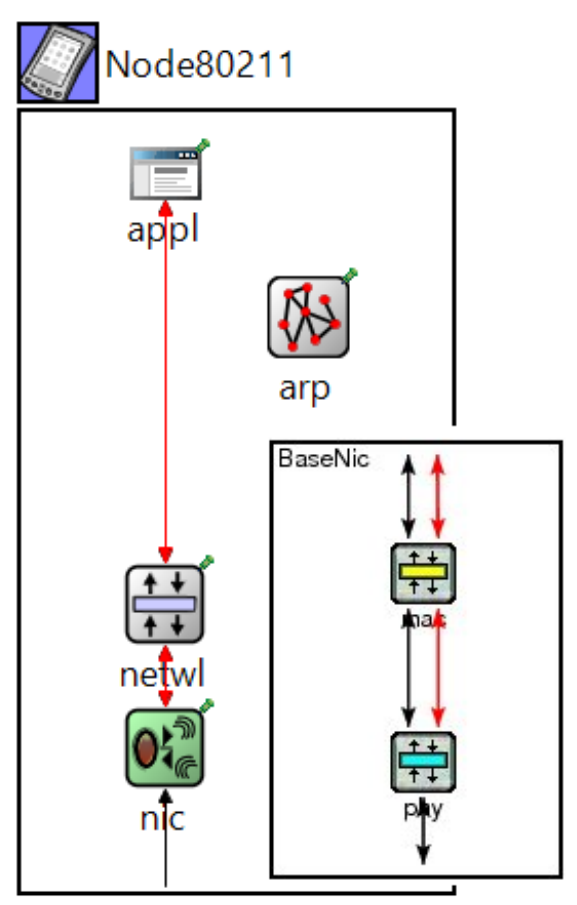

Figure 5.5: The figure represents the MiXiM node model. The green background box labelled "nic" represents the MAC and phy modules. Arp is used by network layer and MAC layer for address resolution. Arrows connecting the sub modules represent connections and gates.

sidering its MAC state, checks on the channel state during every slot time using the getChannelState() to request the phy to sense the channel over a period of time to get the instantaneous channel state. Therefore, each node becomes able to count the number of consecutive idle slots after each transmission attempt and ultimately choose the right $C W$ size.

In order to verify and validate the simulation models various processes and techniques were used to assure the model matches specifications and assumptions with respect to the mathematical model. The behaviour of the design model was checked against the protocols description (cf. \$3.2.1 and 4.1) using OMNeT++'s event log. Figure 5.6 shows the receiving procedure of node 0 in the event log. This procedure start from top of the figure and completed in the button of the figure. As the figure shows the ACK is 


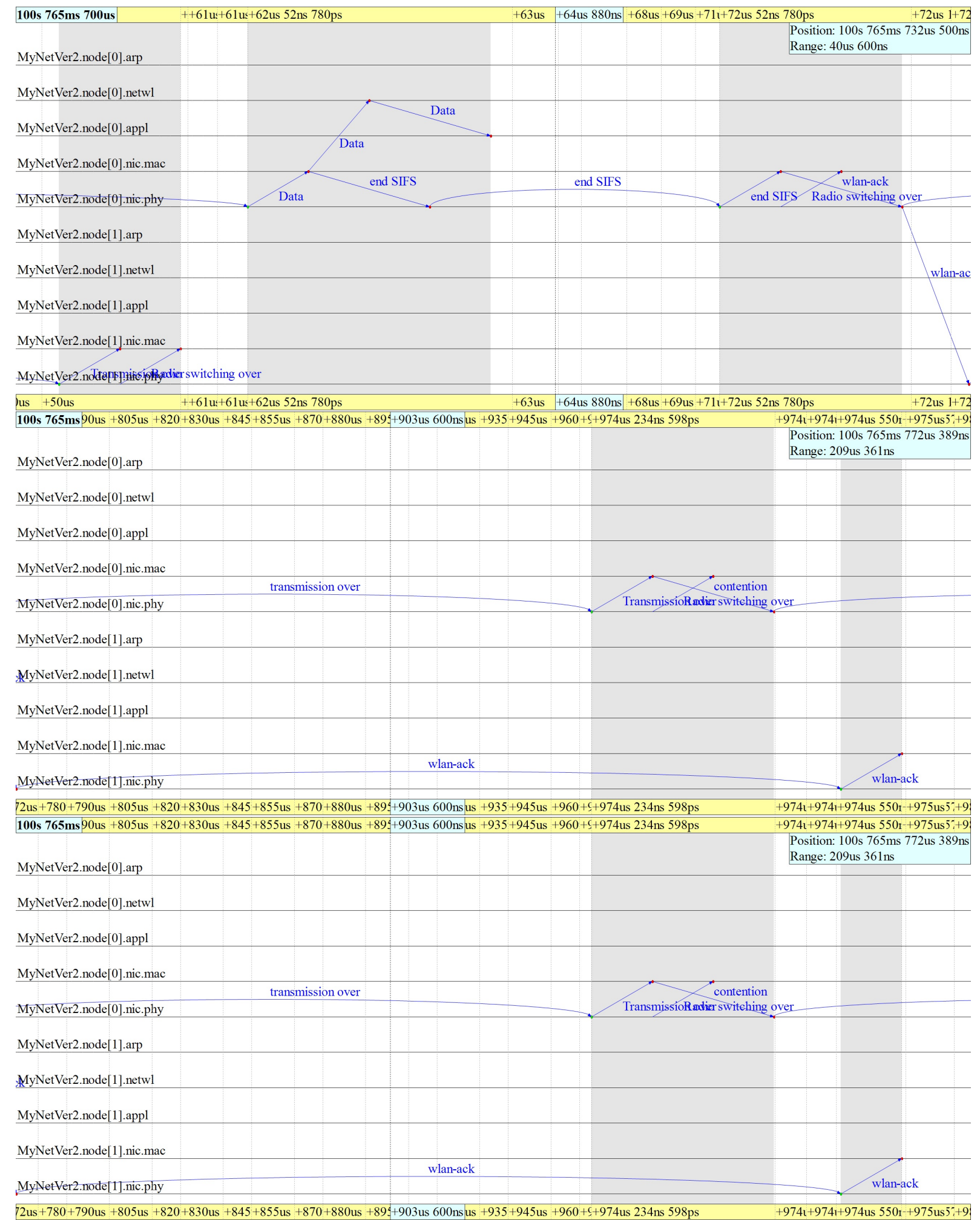

Figure 5.6: The ACK transmission procedure in event log. 
immediately transmitted at the end of the frame transmission, after a SIFS. As the SIFS (plus the propagation delay) is shorter than a DIFS, no other station is able to detect the channel idle for a DIFS until the end of the ACK transmission. This can be seen in the middle image when node 0 detects the channel to be busy and goes into contention resolution mode. (for details of protocol's behaviour please see Figures 3.4 and 3.6 .

In order to verify the simulation results and to assure that they are statistically sound as suggested in [93], the throughput of the mathematical model was compared against simulation output. we compared the mathematical models in [39, 9] with our simulation output, 6.1 for 802.11 /b without hidden terminal and 6.4 and 6.5 for 802.11 in the presence of hidden terminal. The throughput of the mathematical design is slightly better than the simulation models, and the reason is that in mathematical model, the effect of realistic physical channel characteristic (see 2.1) has not been considered. However, the overall behaviour of the simulation matches the theoretical design. For example, as the number of nodes increases, both the theoretical and simulated throughputs decrease almost at the same rate.

\subsection{Metrics}

The proposed game theoretic MAC protocols have a range of interesting properties and features to be analysed and compared to the existing baseline medium access control methods. Statistics of performance metrics were collected periodically for each node during the simulation, which included the number transmitted packets, the number of successfully transmitted packets, the number of collisions, and the size of the MAC buffer. These raw data were processed in MATLAB and the following metrics were computed. 


\subsubsection{Throughput}

Throughput is defined as the successful traffic transmitted between stations. Throughput reflects how the shared medium is utilized by the network's active nodes and quantifies, as a fraction of total channel capacity used successfully. It is given by the following:

$$
\text { Throughput }=\frac{\sum_{i=1, j=1}^{N, P s} P k t_{i j}}{T_{\text {sim }}}
$$

where $P k t_{i j}$ is the $j^{\text {th }}$ packet length of a successful packet received by node $i^{\text {th }} . T_{s i m}$ is the total simulation time in seconds. $N$ is the number of total active nodes in the network. $P s$ is the total number of successful packets received by a node. In this study, "saturation throughput" was considered as main metric to measure the network performance.

This is recognised as an fundamental performance figure, explained as the limit met by the system throughput with the increase of the network traffic. This signifies the maximum traffic the system is able to carry in stable conditions. It is also acknowledged that several random access schemes demonstrate an unstable behaviours; more specifically, with the increase of the offered load, the throughput of random access schemes increases to a maximum value, referred to as "maximum throughput". Nonetheless, subsequent increases of the offered load (traffic) result in a major drop in the throughput of the system. This causes infeasibility in operating the random access scheme at its maximum throughput for a "long" period of time. This instability issue, in terms of its mathematical formulation and interpretation, is the focus of a wide-ranging of discussion in [94]. Indeed, DCF is recognised as showing some type of instability.(see e.g., [95, 96]).

To visualize the unstable behaviour of 802.11 DCF, in Figure 5.7 the simulations have been run in which the offered load linearly increases with the simulation time. The general simulation model and parameters employed are summarized in Appendix C. The 


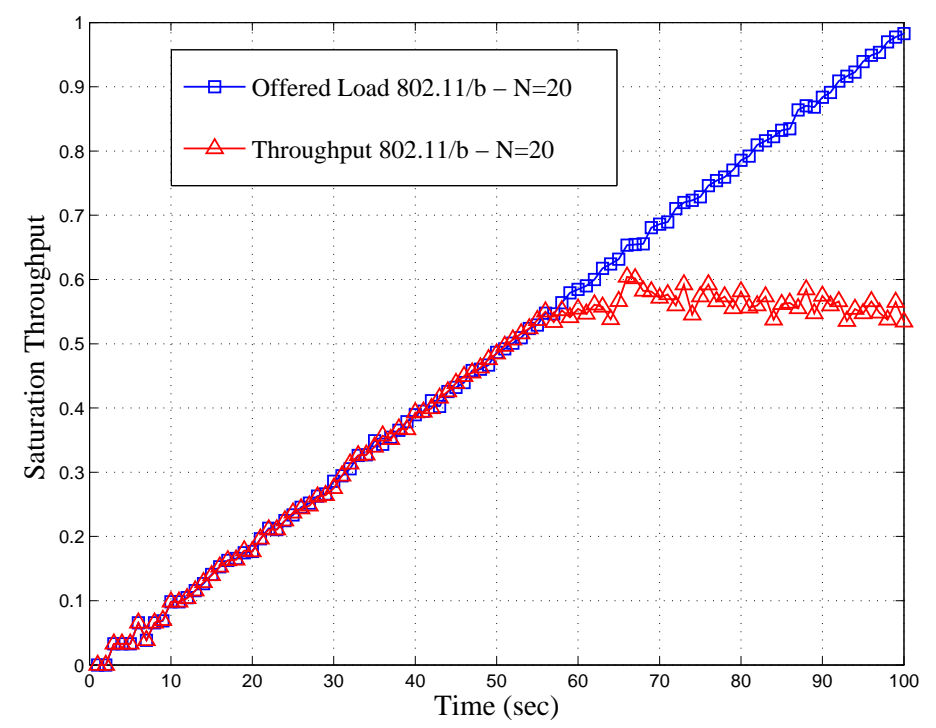

Figure 5.7: Measured throughput with slowly increasing offered load.

throughput depict in Figure 5.7 is obtained with 20 nodes. The simulated offered load has been generated with fixed size packets and is shown by blue line (cf. Table 3.1). The Figure 5.7 shows both offered load and system throughput measured over 100 seconds time intervals and normalized with respect to the channel rate. From the figure, we see that the measured throughput appears to follow closely the measured offered load for the first 58 seconds of simulation, while it asymptotically drops to the value 0.54 in the second part of the simulation run. This asymptotic throughput value is referred to, in this study, as saturation throughput, and represents the system throughput in overload conditions. Note than, during the simulation run, the instantaneous throughput temporarily increases over the saturation value (up to 0.64 in the example considered), but ultimately, it decreases and stabilizes to the saturation value. Queue build-up is observed in such a condition in the MAC layer [9].

\subsubsection{Average delay}

The average delay rate is defined as the aggregate of all MAC layer delays. This was measured by rate of the occupancy of the MAC buffer. The delay rate is given by the 
following:

$$
\text { Delay }_{\text {average }}=\frac{\sum_{i=1}^{N} \frac{\sum_{j=1}^{P s} D e l_{j}}{\text { buffer } \text { size }^{i}}}{N}
$$

Essentially, this means the average of all nodes $\frac{\text { service }_{\text {rate }}}{\text { buffer }_{\text {size }}}$ for specific time interval. Del $l_{j}$ is the $j^{\text {th }}$ packet that has been transmitted by node $i$. Buf $f_{\text {size }} i$ is the occupancy value of MAC buffer for node $i$ over specific time interval.

\subsubsection{Collision rate}

The collision rate of the network measures the collisions that occurred during the simulation time, and it is given by the following:

$$
\text { Collision }_{\text {rate }}=\frac{\sum_{i=1, j=1}^{N, C n} C_{\text {sim }}}{T_{\text {sim }}}
$$

where $\mathrm{Col}_{j}$ is the $j^{\text {th }}$ collided packet detected by node $i$. $T_{\text {sim }}$ is the total simulation time in seconds. $C n$ is total number of collisions that occurred during the simulation time for each node $i$.

\subsubsection{Jain index}

To measure the network fairness, the Jain fairness index was employed, which is defined as the following: [97],

$$
\text { Fairness }=\frac{\left(\sum_{i}^{N} t_{i}\right)^{2}}{|N| \Sigma_{i} t_{i}^{2}}
$$

where $t_{i}$ is the number of transmissions of node $i$ over a specific time window, and $N$ is the total number of active nodes in the network. 


\subsection{Summary}

In this chapter, OMNeT ++ was introduced and chosen as a simulation platform. All the components of the proposed game theoretic MAC protocols are described in the context of OMNeT ++ modelling. A number of components were added to ease simulation debugging, development, and post-processing for metric collection. Significant validation work revealed the necessity of implementation of some parts, including traffic generating, observing idle slot, calculating equilibrium, and impelementing the new backoff function within the application and MAC layers. These parts were fixed and the functionality added, which produced results comparable to published simulation studies for large-scale wireless network in realistic propagation environments. Moreover, the topology design for each proposed MAC protocol was detailed. 


\section{CHAPTER 6}

\section{Simulation ReSUlts}

\subsection{Introduction}

This chapter presents the numerical experiments that evaluate the performance of the proposed medium access method. As discussed in Chapter 5 . OMNeT ++ was employed to implement the game-theoretic medium access protocol, the standard 802.11b (DCF) basic access method, and the RTS/CTS access method. Realistic simulator environment parameters for $802.11 \mathrm{~b}$, such as the physical layer transmit power, receiver sensitivity, etc., were used. The MAC and physical layer information is included in the Appendix C. All other relevant simulation parameters that were used to obtain numerical results in this chapter are listed in Table 3.1 and 6.1 . These system values are those specified in the 802.11b standard with DSSS physical layer [17] and based on the game-theoretic design in Chapters 3 and 4 .

To evaluate the game-theoretic design of Chapter 3 by simulation, a single-cell network without hidden terminals with homogeneous users is considered. All nodes are in saturation mode and also run for varying traffic load, from 0 to 100 percent of the channel capacity. All nodes were located in the OMNet ++ playground using the algorithm detailed in $\$ 5.3 .1$. The size of the network is varied from 5 to 40 nodes in 5 different networks of size 5, 10, 20, 30 and 40 nodes. Each network is run under the DCF MAC protocol 
Table 6.1: Initial game parameters

\begin{tabular}{|c|c|}
\hline Parameters & Value \\
\hline maxtrans for single-cell networks & 5 \\
\hline maxtrans for the networks with hidden terminals & 10 \\
\hline Step size for the gradient play $E\left(\mathbf{P}^{\tau}\right)$ & 0.025 \\
\hline Step size for the gradient play with hidden terminals $E\left(\mathbf{P}^{\tau}\right)$ & 0.020 \\
\hline
\end{tabular}

and then under the game-theoretic protocol. Metrics were collected and are presented in this chapter. Moreover, in networks with hidden terminals present, the simulations were run with different numbers of hidden node, from $0 \%$ hidden nodes up to $60 \%$, using the algorithm explained in $\$ 5.3 .1$. Then the same parameter settings were re-used on the proposed MAC protocol to enable direct comparison. This is done to ensure that fair and representative performance comparisons are made. Also simulations were run for a relatively long time of 1000 seconds to ensure the stability of the protocol designs during the simulation execution time.

\subsection{Saturation Throughput and Collision Rate}

The throughput achieved by the game-theoretic access method compared with $802.11 \mathrm{~b}$ DCF. In the numerical experiments with DCF, after a packet's $(m+1)^{\text {th }}$ failed transmission, the contention window resets to the base contention window, where $m$ denotes the maximum backoff stage, and is set to 5 for $802.11 / \mathrm{b}$. This is also equivalent to the packet being discarded after $m$ failed retransmissions (cf. Figure 3.2).

Figure 6.1 shows the saturation throughput achieved by the game theoretic design and DCF for both access mechanisms. The game-theoretic design achieves the higher saturation throughout in most cases, with only a slightly lower throughput than DCF for a network of a very small number of competing nodes. This also confirms the validity of the approximations (3.2.18) and (3.2.21) made in deriving the utility functions $(3.2 .22)$, and 


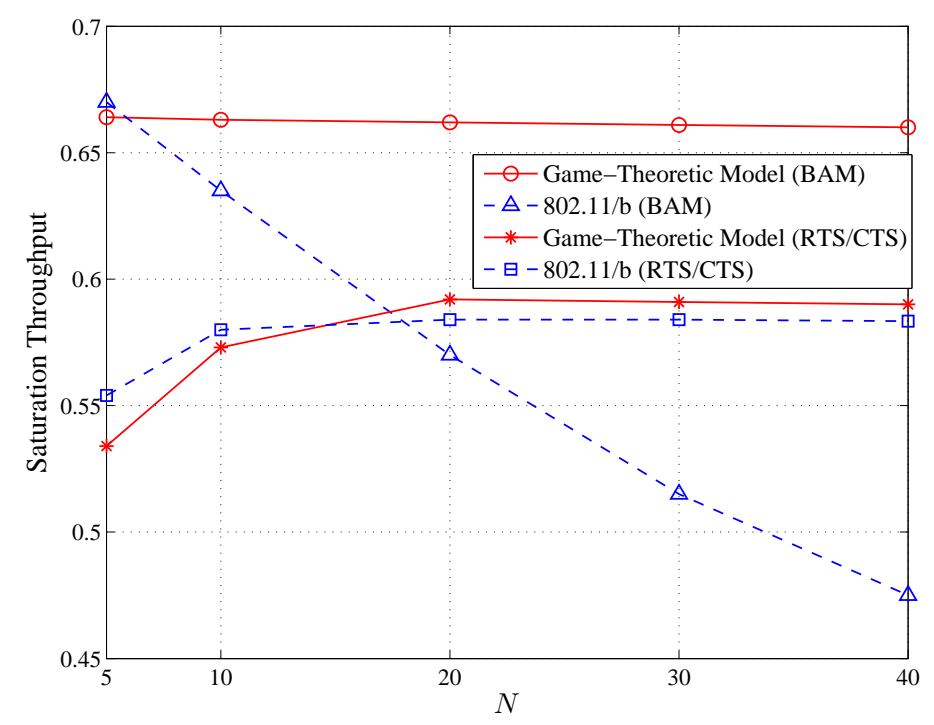

Figure 6.1: Saturation throughput comparison for network of 5-40 competing active nodes operating in basic access and RTS/CTS mechanism.

the contention measure estimator 3.3 .1 and 3.3 .2 gives a fairly accurate estimation of the conditional collision probability. The reason for a lower game-theoretic access scheme throughput for small number of competing nodes is that the utility function (c.f. 3.2 .4 ) is designed based on an optimal number of idle slots for a large number of nodes. $(N \geqslant 10$ in practice, cf. Figures. $3.7 \& 3.8$.

For a network of a very small number of wireless nodes, 802.11 provides a slightly higher throughput than the game-theoretic design. However, with the basic access method as the number of nodes increases, The game-theoretic access method achieves much higher throughput and with RTS/CTS, both designs maintain approximately the same level of performance. With 802.11, each new transmission starts with the base contention window and executes a binary exponential backoff upon collisions, while with the gametheoretic design, nodes choose a constant contention window determined by the Nash equilibrium, which is "optimal" for the current contention level in the network. Thus, for a system of many competing nodes where the contention in the network is heavy, the 802.11 basic access mechanism will incur many more packet collisions than the game- 


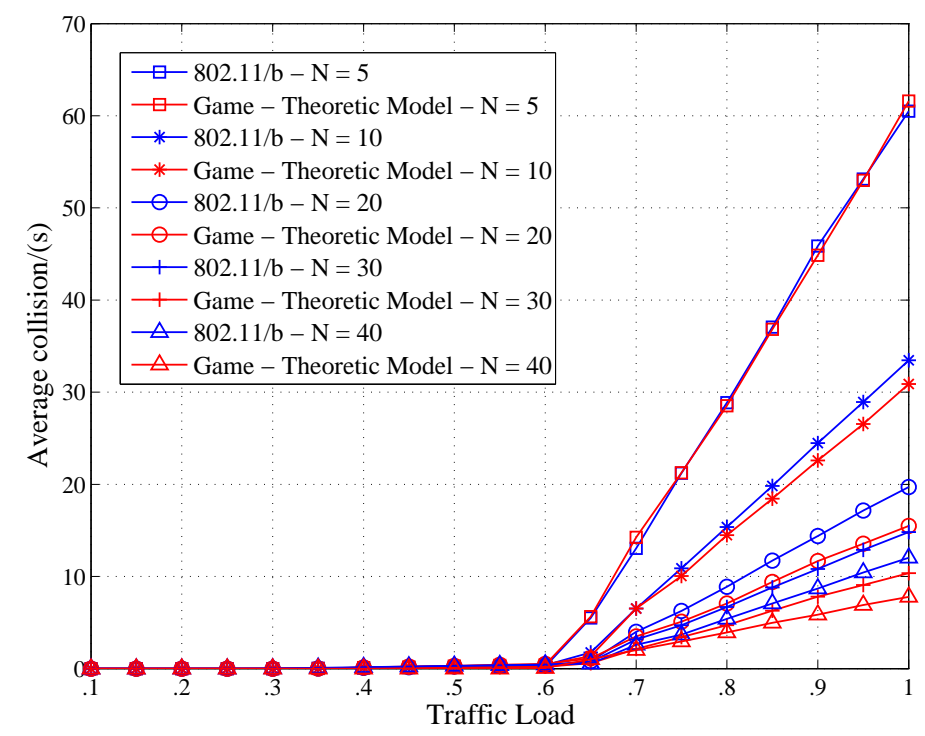

Figure 6.2: Average collision rate comparison for a network of 5-40 active nodes operating in basic access method.

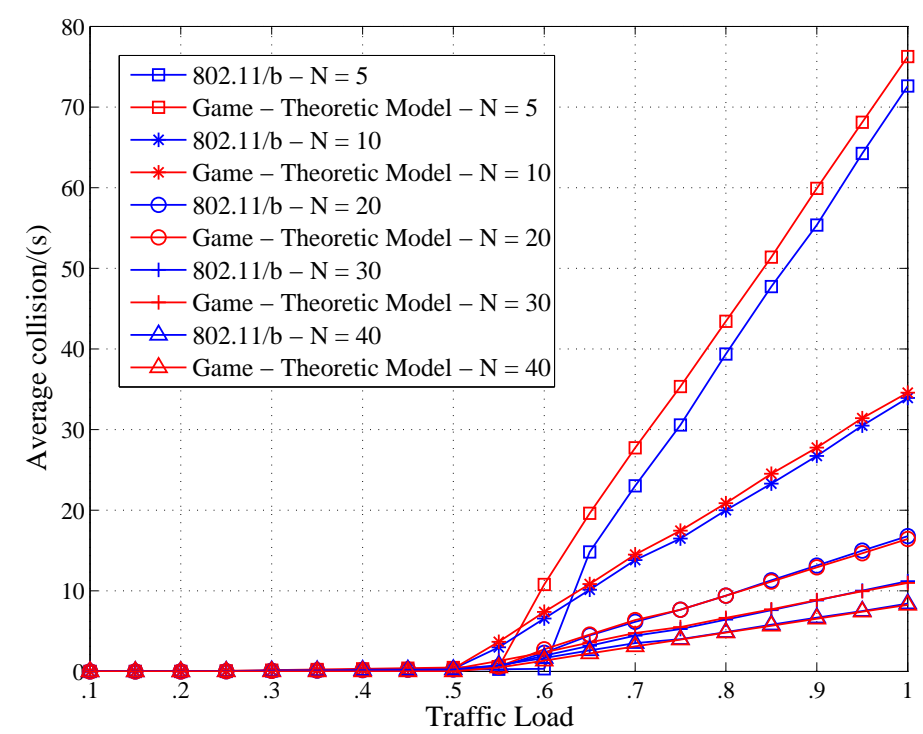

Figure 6.3: Average collision rate comparison for a network of 5-40 active nodes operating in RTS/CTS access method.

theoretic design, which results in a much lower throughput, as shown in Figure 6.1. This is further confirmed by the comparison of collision overheads, as shown in Figure 6.2. The 802.11 RTS/CTS increases the system performance by reducing the duration of a collision (the vulnerable period) when data frames are transmitted. On the other hand, 


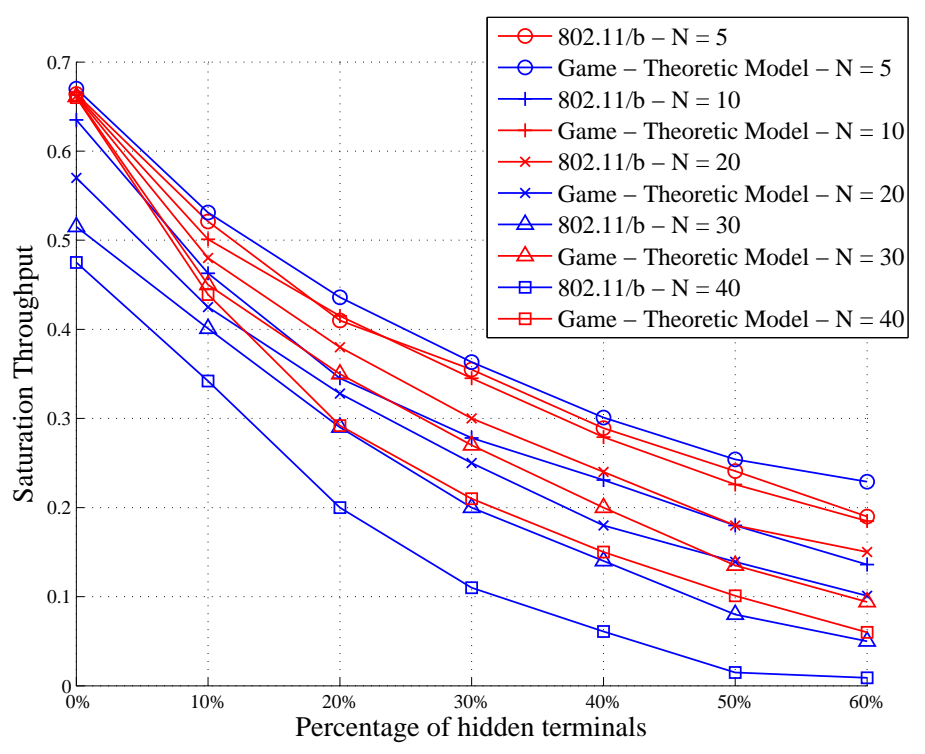

Figure 6.4: Saturation throughput comparison for network of 5-40 competing active nodes and with different percentage of hidden terminals, operating in basic access.

RTS/CTS decreases efficiency since it transmits two additional frames without any payload, which result in lower performance than the game-theoretic design in the basic access method. The game-theoretic design both in basic access and RTS/CTS achieves a very small, almost constant collision rate (Figures 6.2 \& 6.3), better tradeoff between channel access and collision avoidance, and hence a higher throughput that is sustainable over a large range of numbers of competing nodes. Practically, this means that the game theoretic design can achieve a higher throughput, but with fewer transmissions than 802.11 DCF, which will benefit the whole system in many aspects, such as having lower energy usage and less interference to the wireless nodes of neighbouring cells, if any exist.

As mentioned in the introduction, simulations for the networks with hidden terminals were run for different numbers of nodes and different percentages of hidden terminals. Figure 6.4 shows the saturation throughput achieved by the game theoretic design and the 802.11 basic access method. Figure 6.5 shows the saturation throughput of the game theoretic design and the 802.11 RTS/CTS access method. The game-theoretic design achieves the higher saturation throughout for large networks with only a slightly lower 


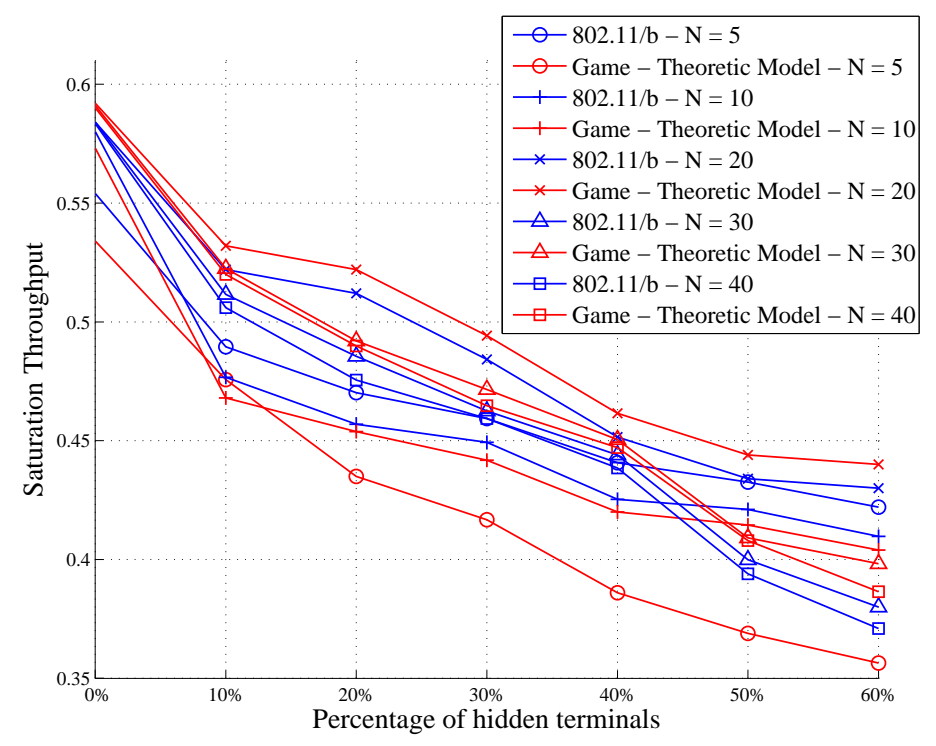

Figure 6.5: Saturation throughput comparison for network of 5-40 competing active nodes and with different percentage of hidden terminals, operating in RTS/CTS mechanism.

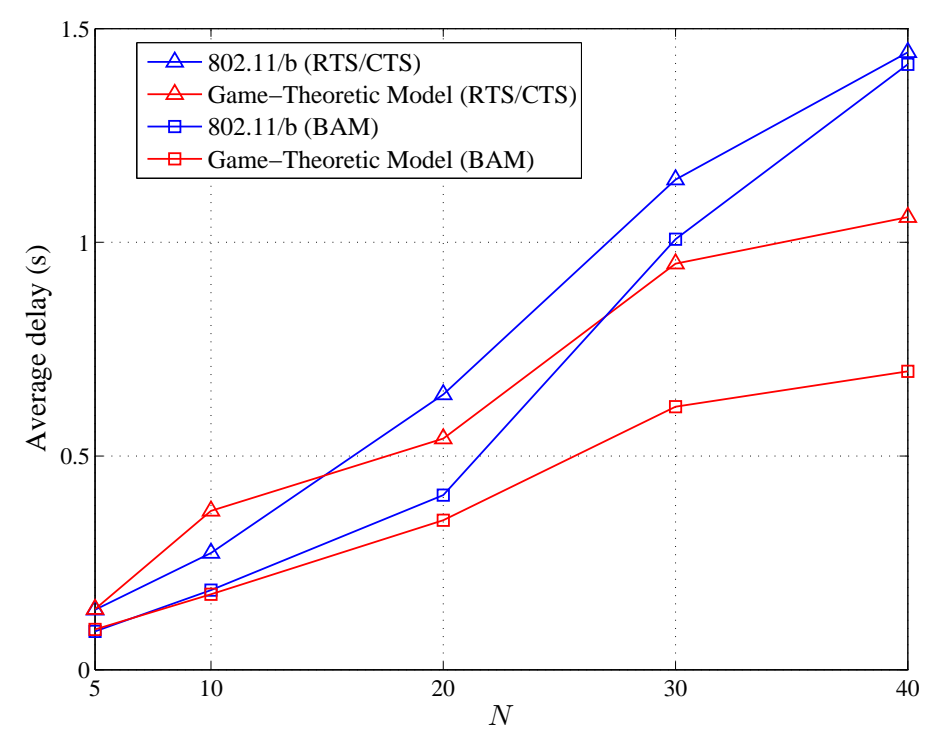

Figure 6.6: Average delay comparison for a network of 5-40 active nodes, operating in RTS/CTS and basic access method (DCF \& game-theoretic design).

throughput than DCF for a network of a very small number of competing nodes. This also confirms the validity of the approximations 4.2 .20 and 4.2 .23 made in deriving the utility functions (4.2.24), and the contention measure estimator 4.3.1) and (4.3.2) gives a fairly accurate estimation of the conditional collision probability. Again the reason for 
a lower game-theoretic access scheme throughput for small number of competing nodes is that the utility function is designed based on a Markovian model [9, 39] which is accurate for the networks with the size of $N \geqslant 10$.

Compared to 802.11, for a network of a very small number of wireless nodes with hidden terminals and AP as central coordinator in the network, 802.11 provides a slightly higher throughput than the game-theoretic design for different percentages of hidden nodes. However, with DCF operating in basic access method, as the number of nodes increases, the game-theoretic access method achieves a much higher throughput, especially when the percentage of hidden terminals increases in the network. In networks operating in RTS/CTS mechanism both designs (game-theoretic and DCF) again keep almost the same level of performance. With 802.11, each new transmission starts with the base contention window and executes binary exponential backoff upon collisions, while, with the game-theoretic design nodes choose a constant contention window determined by the Nash equilibrium, which is "optimal" for the current contention level in the network. Thus, for a system of many competing nodes with hidden terminals where the contention in the network is heavy, the 802.11 basic access mechanism will incur much more packet collisions than the game-theoretic design, which results in much lower throughput, as shown in Figure 6.4.

This is further confirmed by the comparison of collision overheads for different percentages of hidden terminals in each network topology, as the lower part of each sub figure shown in Figure 6.7. The game-theoretic design both in basic access and RTS/CTS achieves a lower collision rate for a system of many competing nodes (Figures 6.7 \& 6.8), i.e., a better tradeoff between channel access and collision avoidance, and hence a higher throughput that is sustainable over a large range of numbers of competing nodes, as the number of hidden nodes increases. Practically, this means that game theoretic design can achieve higher throughput, but with fewer transmissions than 802.11 DCF, the same argument 
which was made in single-cell network with same benefits, which are lower energy usage and less interference to the wireless nodes of neighbouring cells. Figures $6.7 \& 6.8$ also exhibit the power of four-way hand shaking of the RTS/CTS access method in dealing with hidden terminals in the network by reducing the number of collisions significantly. the warmer colour shows more collisions and cooler colour show less collisions. Top part of each sub-figure shows the collision rate of DCF and the bottom part shows the collision rate of the game.

The Figure 6.4 shows, as the percentage of hidden terminals in the network increases, the saturation throughput of the DCF basic access method dramatically decreases: E.g., for network of size of 40 nodes as the percentage of hidden terminals reaches $60 \%$ the saturation throughput almost approaches zero. In contrast to DCF operating in the RTS/CTS mode, where the RTS/CTS handshaking manages to keep the throughput at approximately 0.37 (cf. Figure 6.5), another observation worth mentioning is that the level of the game-theoretic design throughput improvement is more significant in the basic access method than in the RTS/CTS access method. This is because of the level of de-synchronization among the hidden nodes in the DCF basic access method (cf. \$4.1.2). Therefore, the game-theoretic MAC protocol is able to improve the system performance more effectively than the RTS/CTS access method, which already has the appropriate tools to deal with this de-synchronization, to reduce the vulnerable period among hidden nodes (cf. \$4.1.3).

\subsection{Average Channel Access Delay}

For $802.11 / \mathrm{b}$ (for both the RTS/CTS \& basic access mechanisms), a network of a very small number of wireless nodes provides a slightly lower difference in channel access delay than the game-theoretic design (cf. Figure 6.6). As mentioned in the above sections, this is because design of the utility function based on a Markov model is only accurate 
for networks with size $N \geqslant 10$. However, as the size of the network increases, the gametheoretic design provides a much lower channel access delay. Moreover, RTS/CTS both in DCF and the game-theoretic design produce longer delays because of the four-way hand shake which produces extra packets without payload for time synchronisation among nodes. Figures 6.9, 6.10, 6.11, and 6.12 compare the DCF delay distribution of the basic and RTS/CTS access methods with their corresponding game-theoretic design over 1000 second simulation runs. The left sub-figure for each network shows the linear scale delay distribution histogram and the right sub-figure shows the logarithmic scale delay histogram of the left sub-figure to emphasize long delays with small but finite probabilities. With 802.11, each new transmission starts with the base contention window and executes a binary exponential backoff when collisions occur, while with the game-theoretic design, nodes choose a constant contention window determined by the Nash equilibrium, which is "optimal" for the current contention level in the network. Therefore, in the game-theoretic design the delays are tightly distribute around a specific range of times on the other hand, the delay in DCF is more broadly distributed and exhibits long tails. As the size of the network increases, this distribution for DCF becomes wider. This demonstrates that the game-theoretic design has better short-time fairness (this will be discussed in more detail in the next section).

Figures 6.13 and 6.14 compare the average channel access delay of the two protocols for different percentages of hidden terminals in the network. It can be seen that the gametheoretic design provides a much better channel access delay than DCF. Figures B.1 B.19 compare the normalized delay distribution of the two protocols for each network depicted in Figures 6.13 and 6.14 . The large histogram peak at the right hand side of the rightmost sub-plots shows that 802.11 aggressively moves toward the maximum contention window size $C W_{\text {max }}$, but the game theoretic model adjusts its CW based on the Nash equilibrium in a much more measured way. In the networks with hidden terminals present, and which 
are operating in the RTS/CTS access method, all stations are adjusting their NAV based on the duration field value of the RTS from the source station, or the CTS from the destination station. The use of RTS/CTS frames helps to minimise the duration of the collisions, including those caused by hidden stations. More specifically, if a collision occurs with two or more small RTS frames, the time loss is smaller compared to the collision of long data frames. Furthermore, the successful exchange of small messages, RTS and CTS, reserves the area within the range of the receiver and the sender for the intended transmission period guaranteeing undisturbed transmission for the longer data frame, which results in lower delay, better fairness and higher throughput.

\subsection{Fairness}

It is well-known [48] that the DCF backoff scheme (binary exponential backoff) has poor short-time fairness. This is the case for two reasons. First, a node that fails to transmit successfully has to wait for a longer period of time before it retries. Second, whenever a failed transmission is reported, DCF automatically considers it as a collision, and therefore, it executes a binary exponential backoff with similar results. Figures 6.15 and 6.16 compare the short-time fairness of the game-theoretic model and DCF, using the Jain fairness index [97].

The game-theoretic access scheme in both the basic and RTS/CTS access methods produces a much better short-time fairness than the IEEE 802.11b DCF for networks of many competing nodes $(N \geqslant 10)$, as expected due to the sharing of the same contention window by all the nodes in single-cell networks, which results in the same channel access probability for all nodes. On the other hand for DCF, a node that fails to transmit successfully has to wait for a longer period of time before it retries, and whenever a failed transmission is reported DCF automatically considers it as a collision. Therefore, it executes a binary exponential backoff which results in dramatic differences in CW sizes 
between nodes. This difference becomes rapidly larger as the number of nodes in the network increases, which ultimately leads to lower short-time fairness, which can imply lower long-time fairness as well.

As expected, the 802.11 RTS/CTS mechanism provides the better short-time fairness in a network without hidden terminals than the basic access method by introducing extra packets for synchronisation between nodes to reduce the vulnerable time (cf. Figure 4.4). This results in reducing the duration of a collision and ultimately yield better shorttime fairness. However, the true power of the RTS/CTS access method reveals itself in networks with hidden terminals present. Figures 6.17 \& 6.18 show that RTS/CTS has much better short-time fairness than the basic access method. These figures illustrate how short-time fairness drops below 0.1 in the basic access method in a network of 40 nodes, but with RTS/CTS the fairness is maintained above 0.6. Also, in both access methods, the game-theoretic design has much better fairness than DCF. This is because each node in the game-theoretic design adopts the "optimal" contention window based on the number of hidden terminals that particular node interact with. Therefore, if a node interacts with more hidden terminals, it has to choose a bigger contention window, and this results in nodes with higher number of hidden terminal in their neighbours staying more "quiet" and allow the overall network to operate better. Superficially, it seems this might result in unfairness, but in comparison with DCF, the game-theoretic design shows that this choice of contention window will result in better fairness because it decreases the collision probability across the entire network. This ultimately means, even nodes with higher numbers of hidden terminal neighbours have more chance to transmit their data packets successfully than DCF, and do so more frequently. 


\subsection{Conclusion}

This chapter illustrates that 802.11 starts each new transmission with the base contention window, and after each collision, it executes a binary exponential backoff. As the size of a single-cell network grows, or the percentage of hidden terminal increases, 802.11 aggressively moves toward the maximum contention window $C W_{\max }$. Thus, the binary exponential backoff collision avoidance mechanism leads to longer delays, unfairness among the nodes and increasing the collision probability by not considering the vulnerable period among the covered and especially hidden nodes, which decreases the whole network performance. While, on the contrary, the game-theoretic design nodes choose a constant contention window determined by the Nash equilibrium, which is "optimal" for the current contention level in the network, both for single-cell network and for network with hidden terminals present. This decreases the collision probability, which leads to better fairness, lower delays, and ultimately, better network performance. 


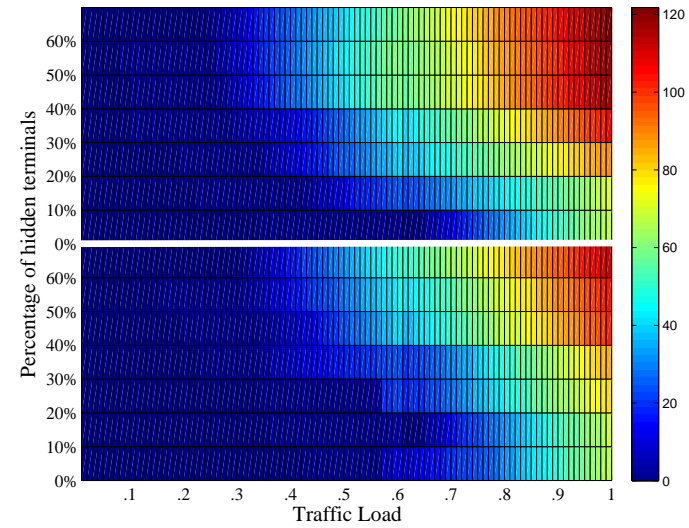

(a) $N=5$

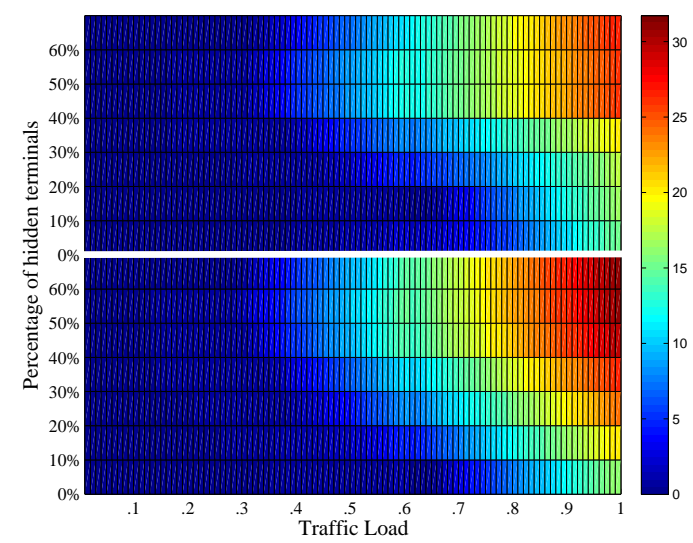

(c) $N=20$

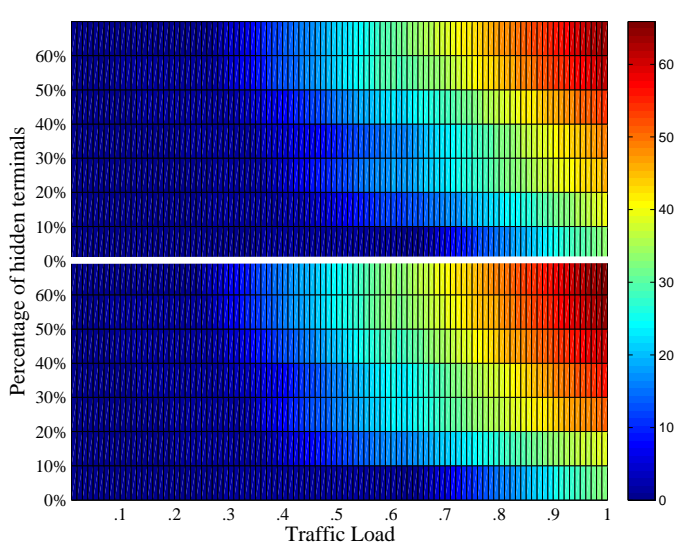

(b) $N=10$

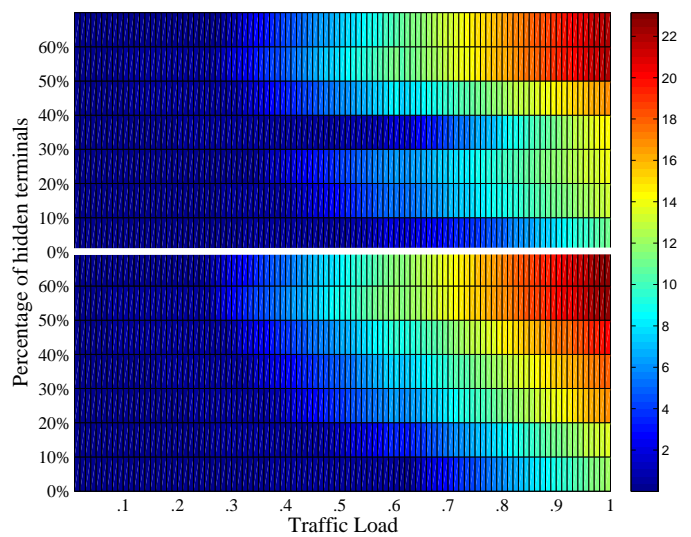

(d) $N=30$

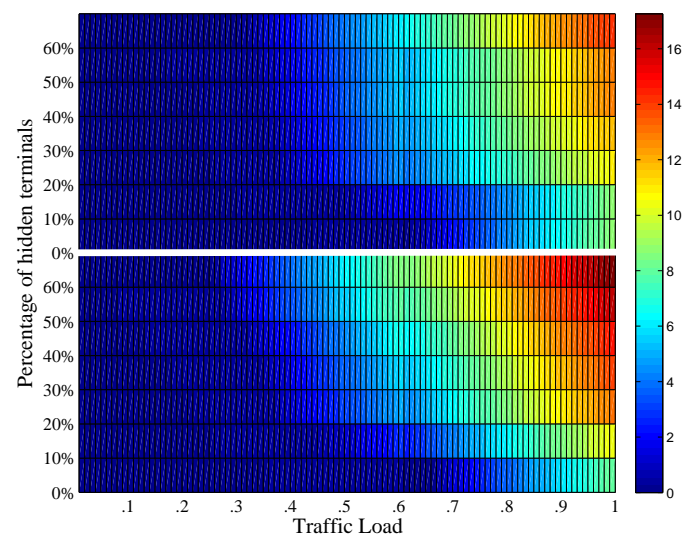

(e) $N=40$

Figure 6.7: Average collision rate comparison for a network of 5-40 active nodes operating in basic access (DCF \& game-theoretic design) method with 0-60 percent hidden terminal for each scenario. 


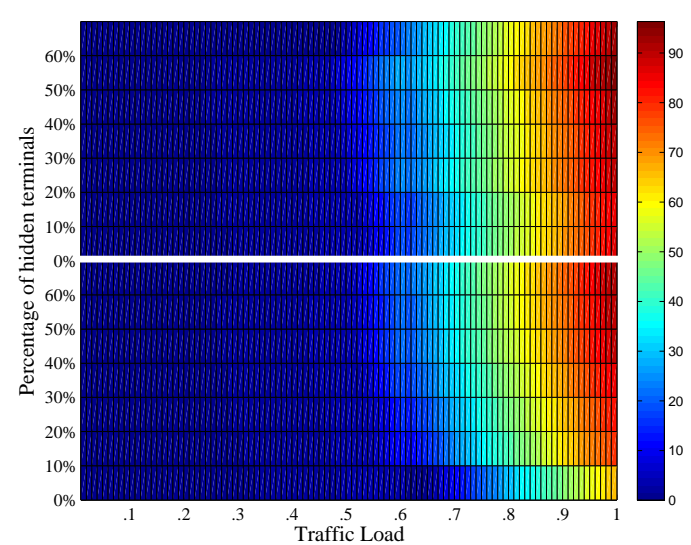

(a) $N=5$

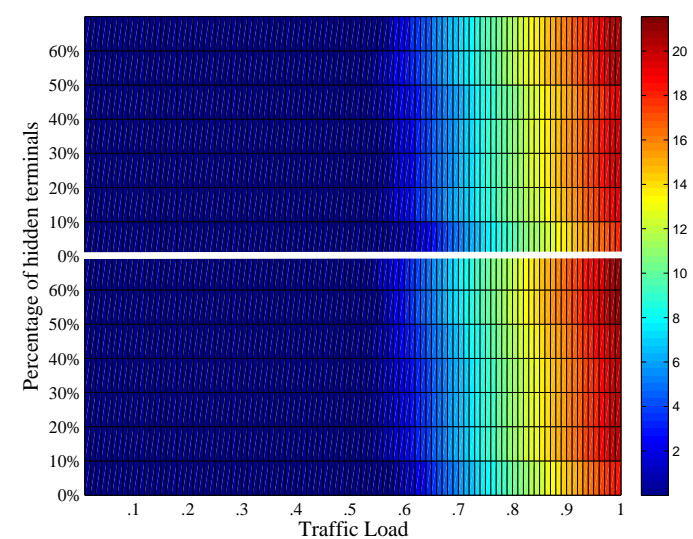

(c) $N=20$

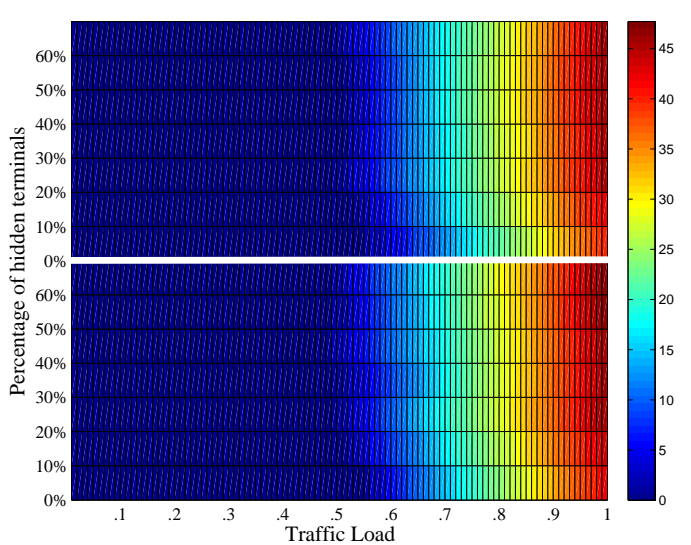

(b) $N=10$

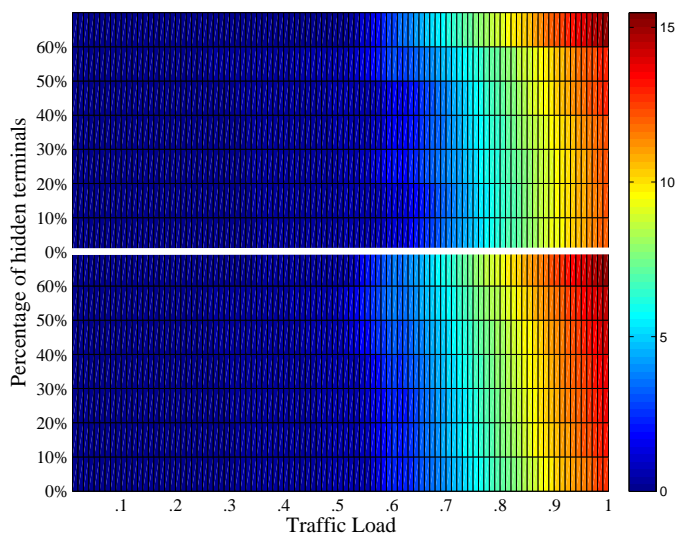

(d) $N=30$

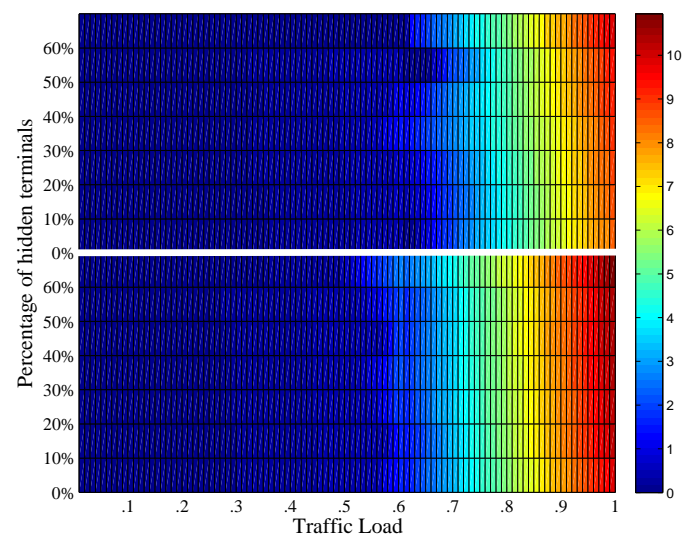

(e) $N=40$

Figure 6.8: Average collision rate comparison for a network of 5-40 active nodes operating in RTS/CTS access (DCF \& game-theoretic design) method with 0-60 percent hidden terminal for each scenario. 


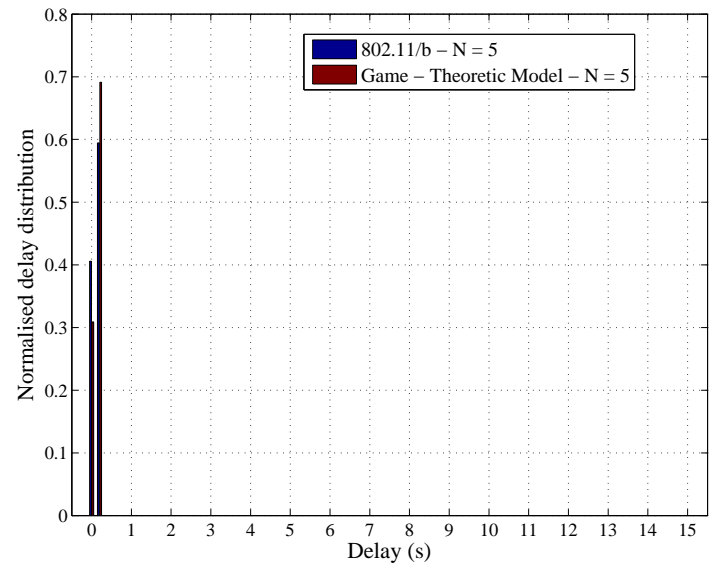

(a) Linear scale

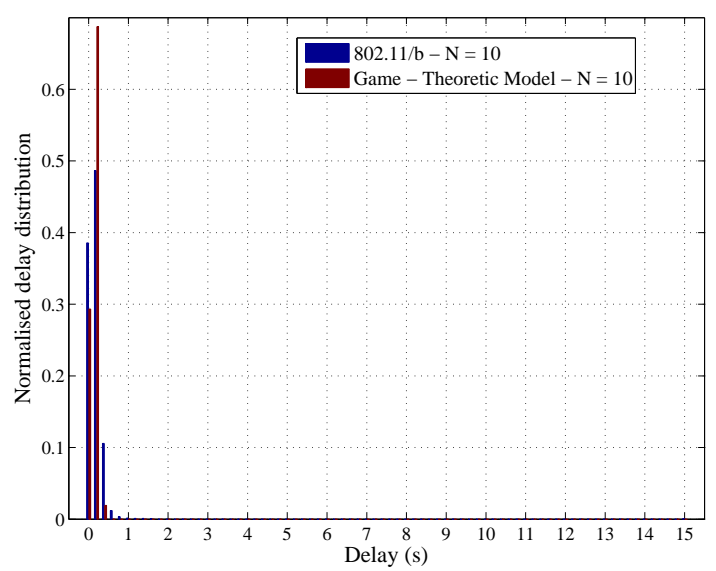

(c) Linear scale

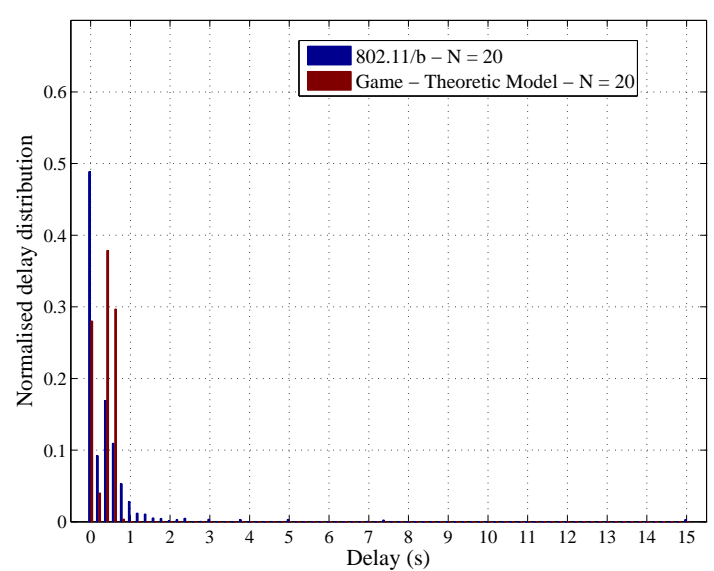

(e) Linear scale

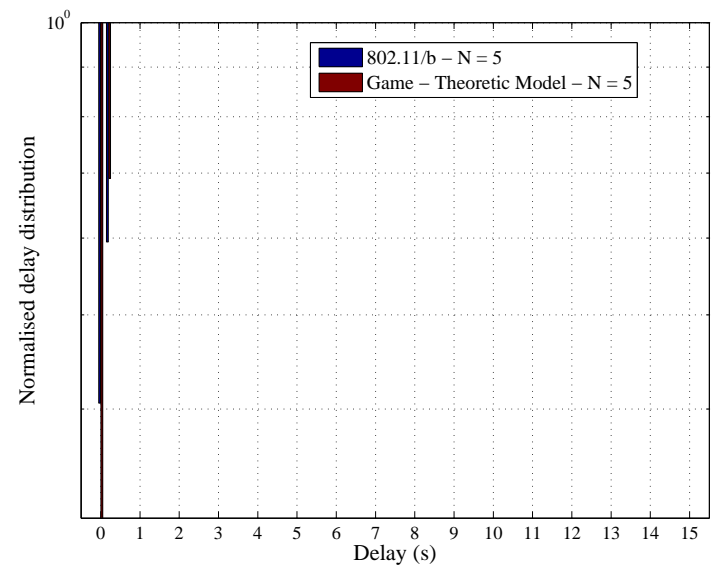

(b) Logarithmic scale

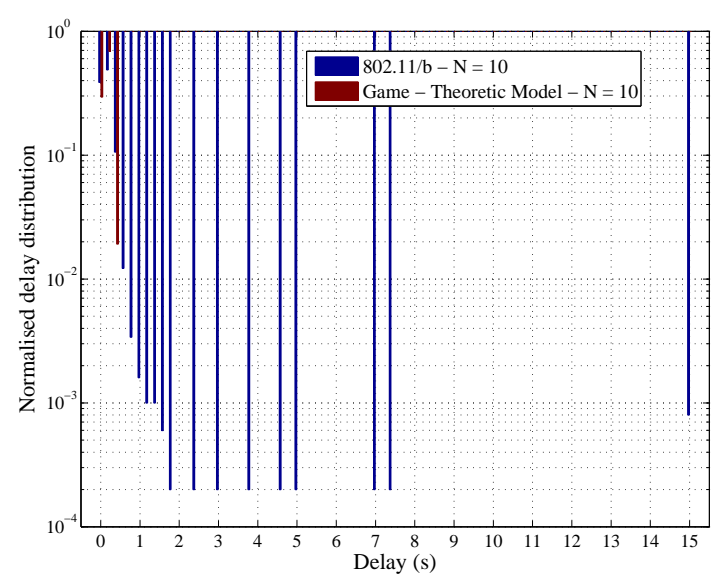

(d) Logarithmic scale

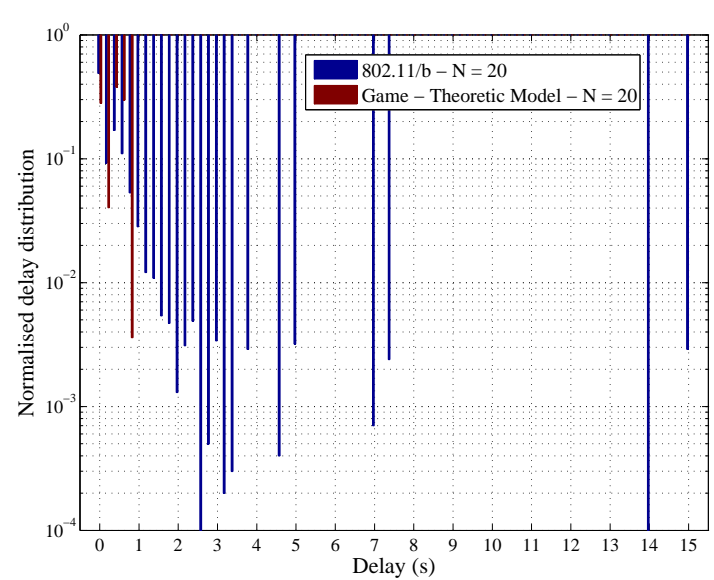

(f) Logarithmic scale

Figure 6.9: Normalised delay distribution comparison for a network of 5-20 active nodes, operating in basic access method (DCF \& game-theoretic design). The bin-size is 0.2 seconds. 


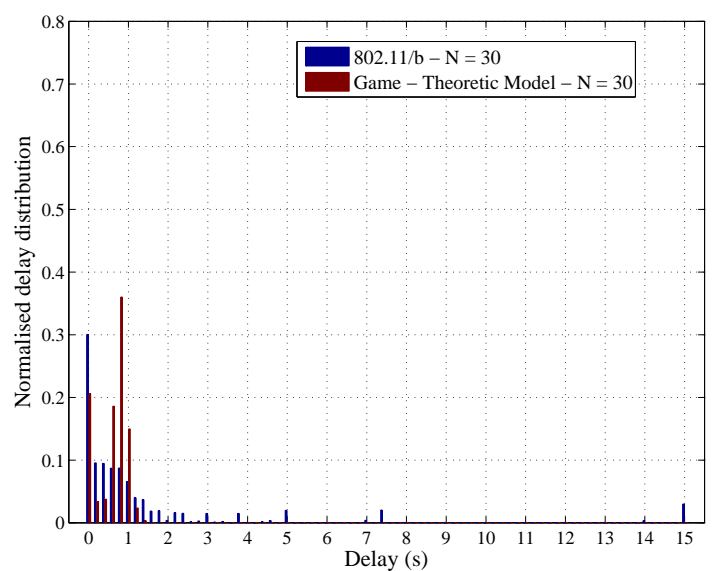

(a) Linear scale

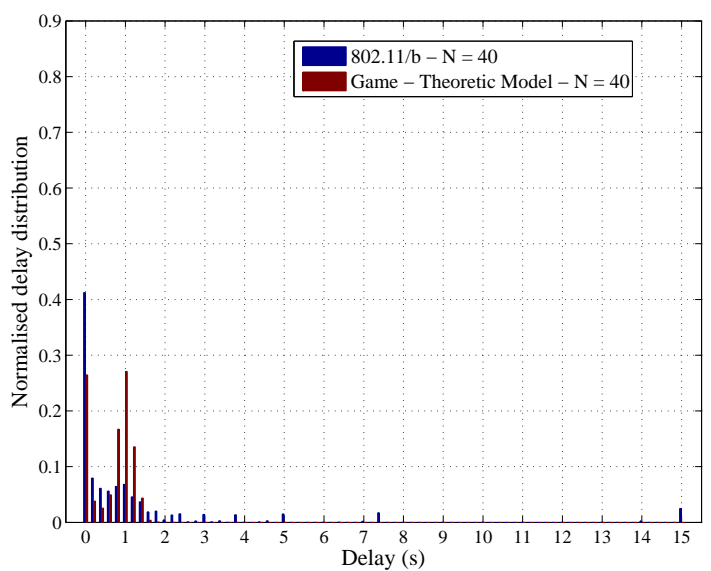

(c) Linear scale

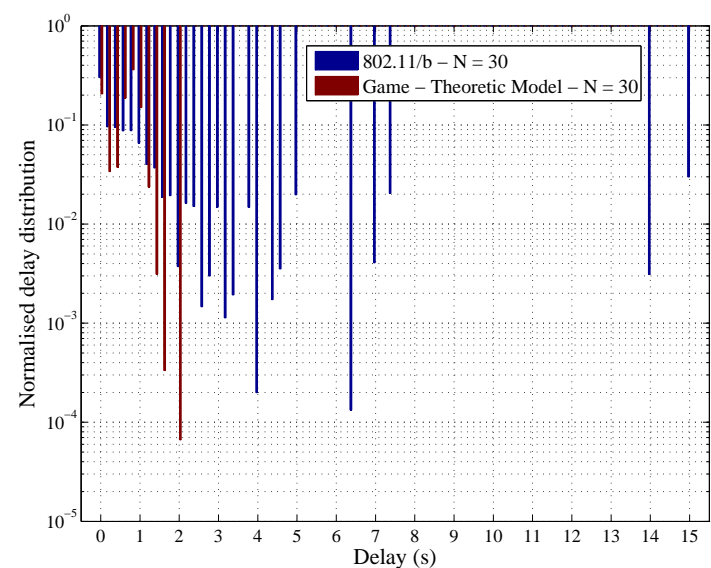

(b) Logarithmic scale

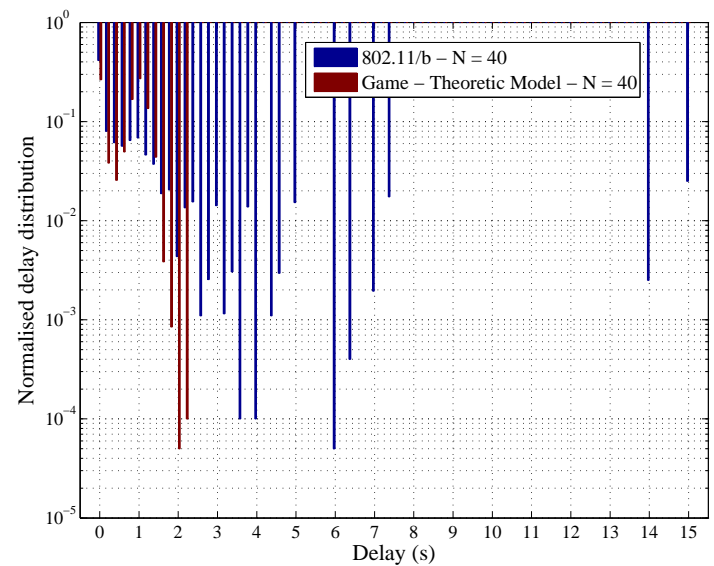

(d) Logarithmic scale

Figure 6.10: Normalised delay distribution comparison for a network of 30-40 active nodes, operating in basic access method (DCF \& game-theoretic design). The bin-size is 0.2 seconds. 


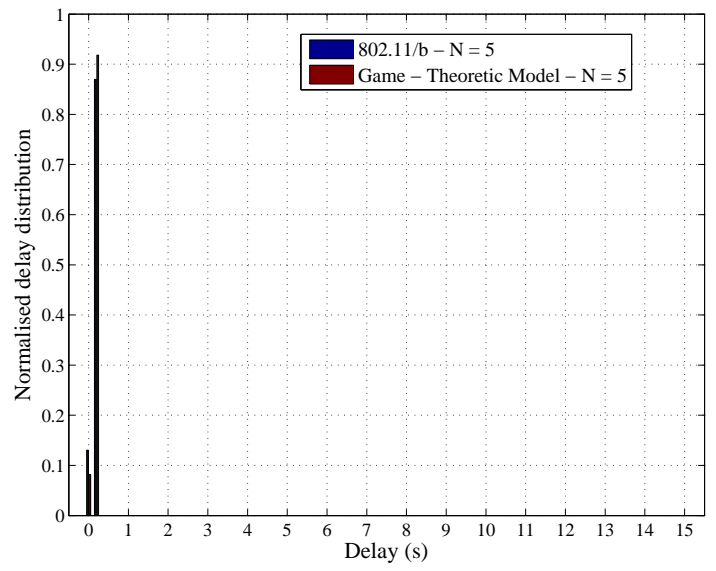

(a) Linear scale

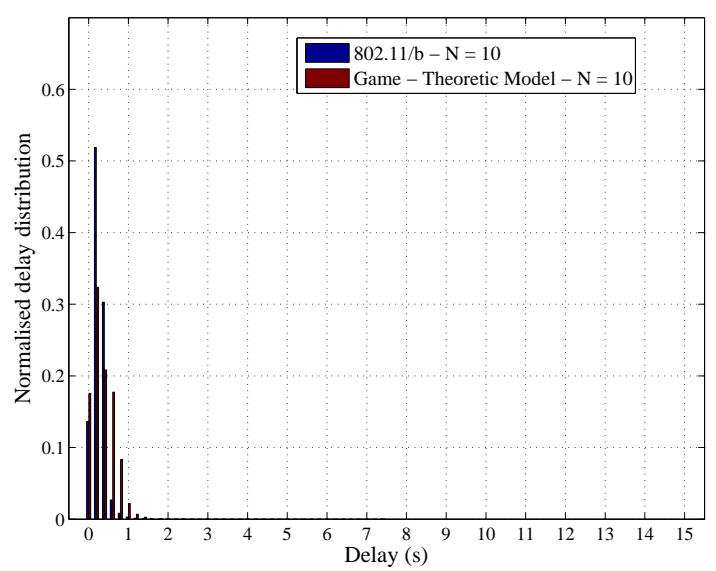

(c) Linear scale

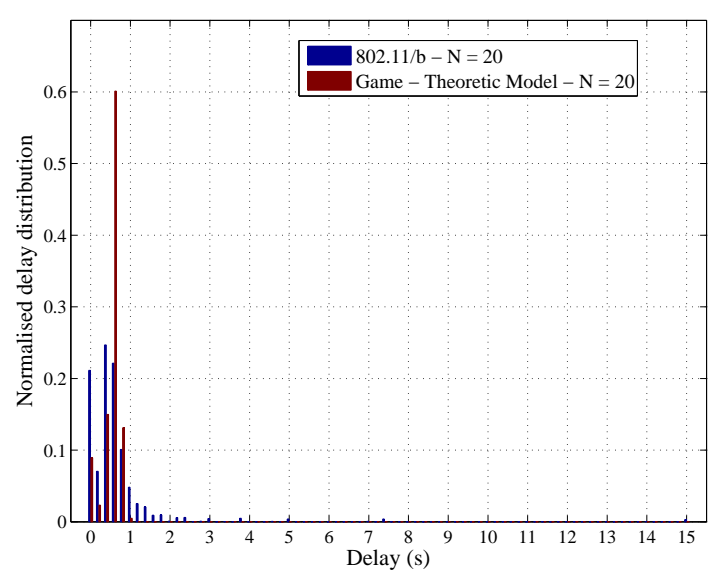

(e) Linear scale

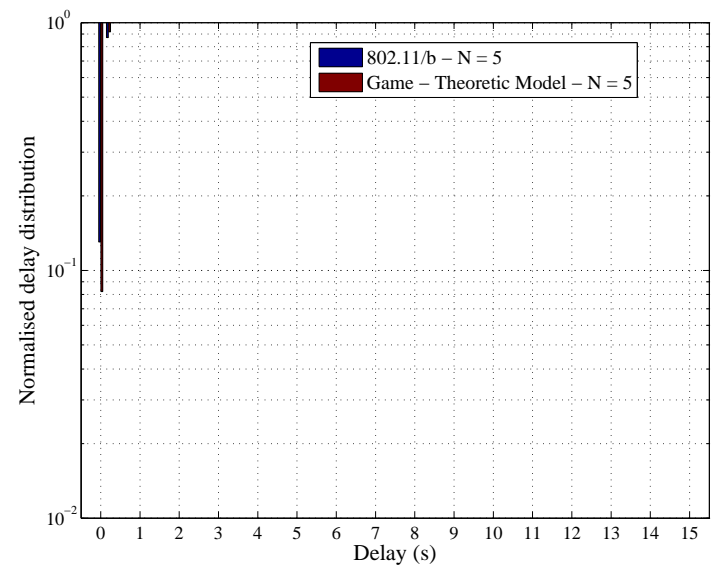

(b) Logarithmic scale

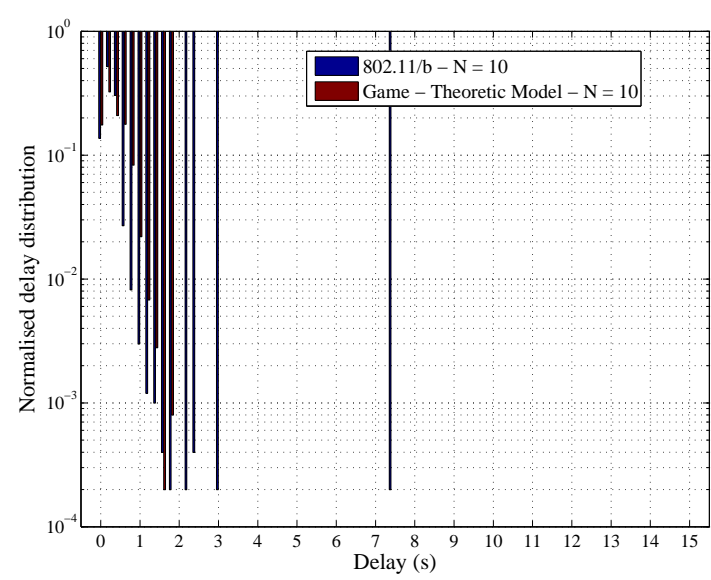

(d) Logarithmic scale

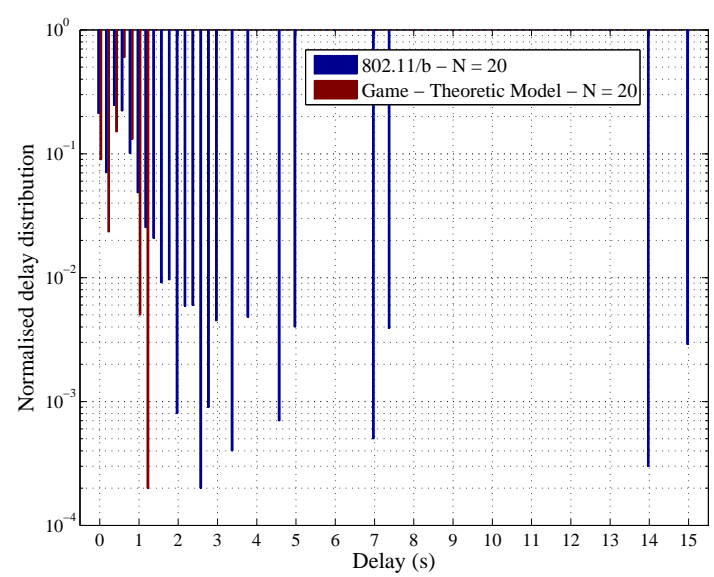

(f) Logarithmic scale

Figure 6.11: Normalised delay distribution comparison for a network of 5-20 active nodes, operating in RTS/CTS access method (DCF \& game-theoretic design). The bin-size is 0.2 seconds. 


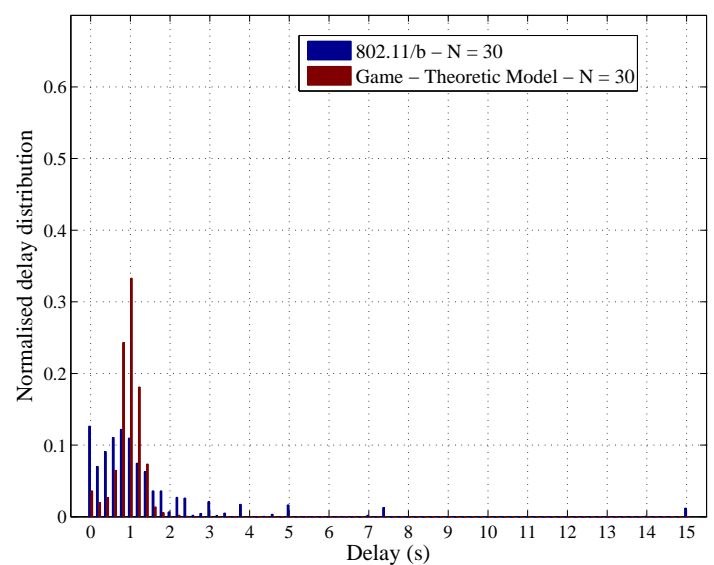

(a) Linear scale

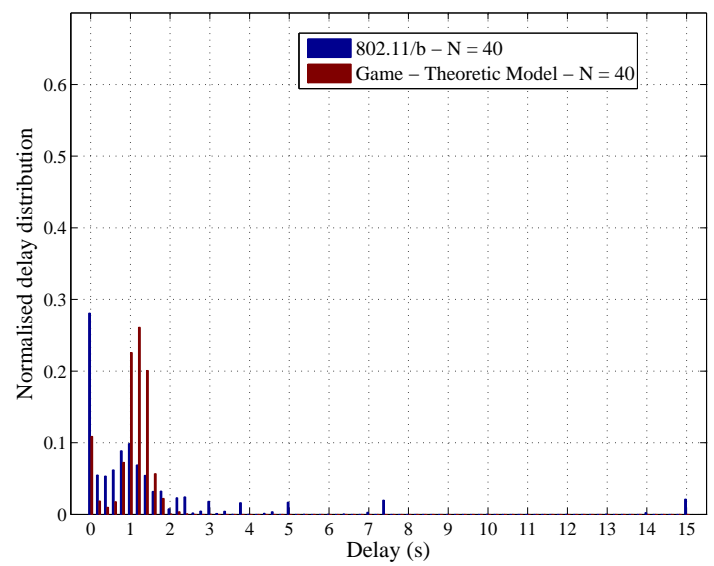

(c) Linear scale

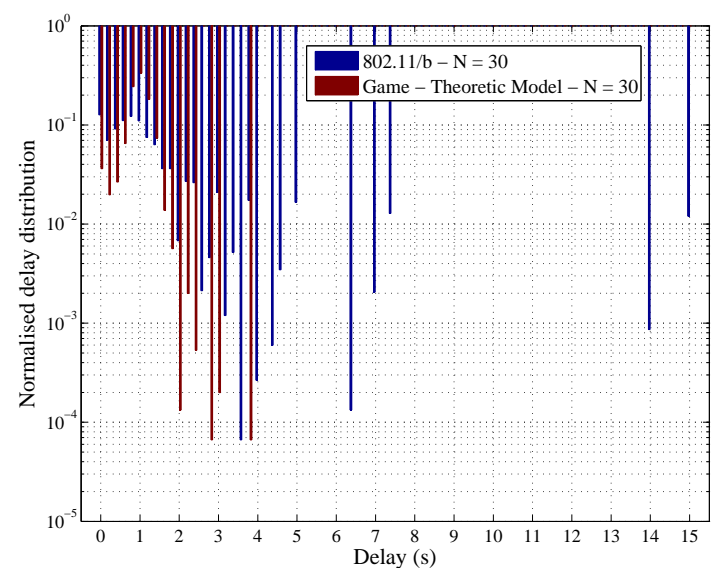

(b) Logarithmic scale

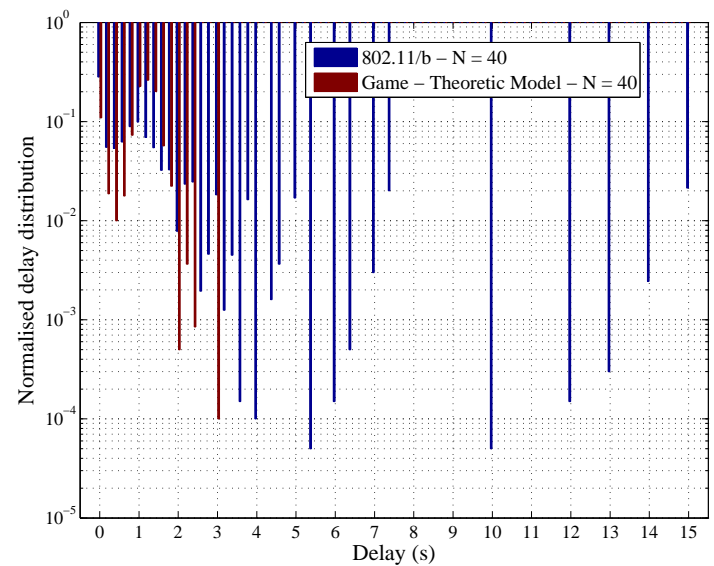

(d) Logarithmic scale

Figure 6.12: Normalised delay distribution comparison for a network of 30-40 active nodes, operating in RTS/CTS access method (DCF \& game-theoretic design). The bin-size is 0.2 seconds. 


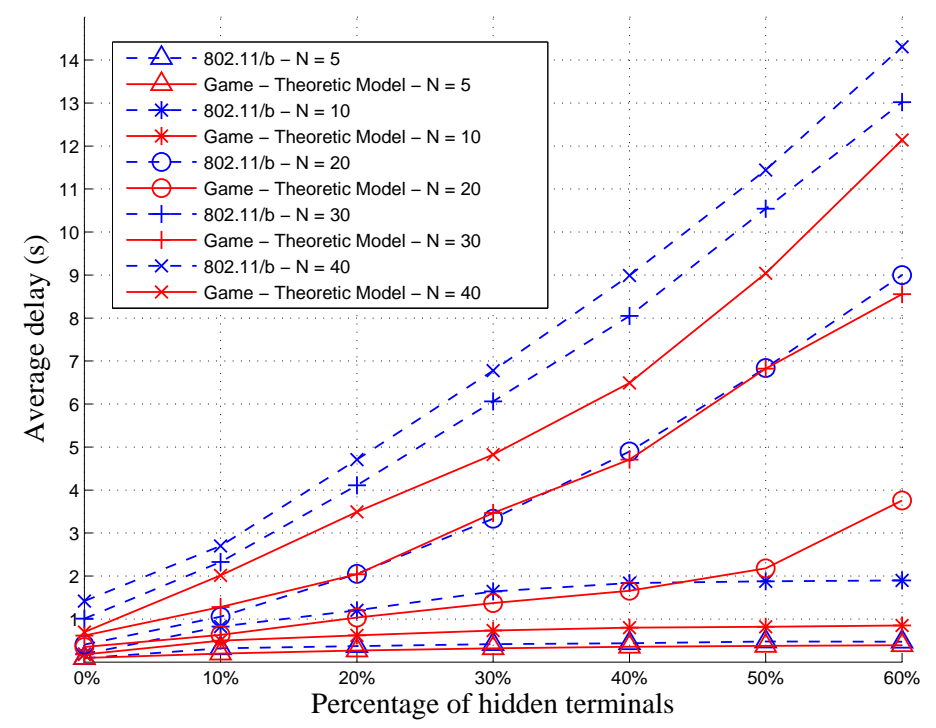

Figure 6.13: Average delay comparison for a network of 5-40 active nodes operating in basic access (DCF \& game-theoretic design) method with 0-60 percent hidden terminal for each scenario

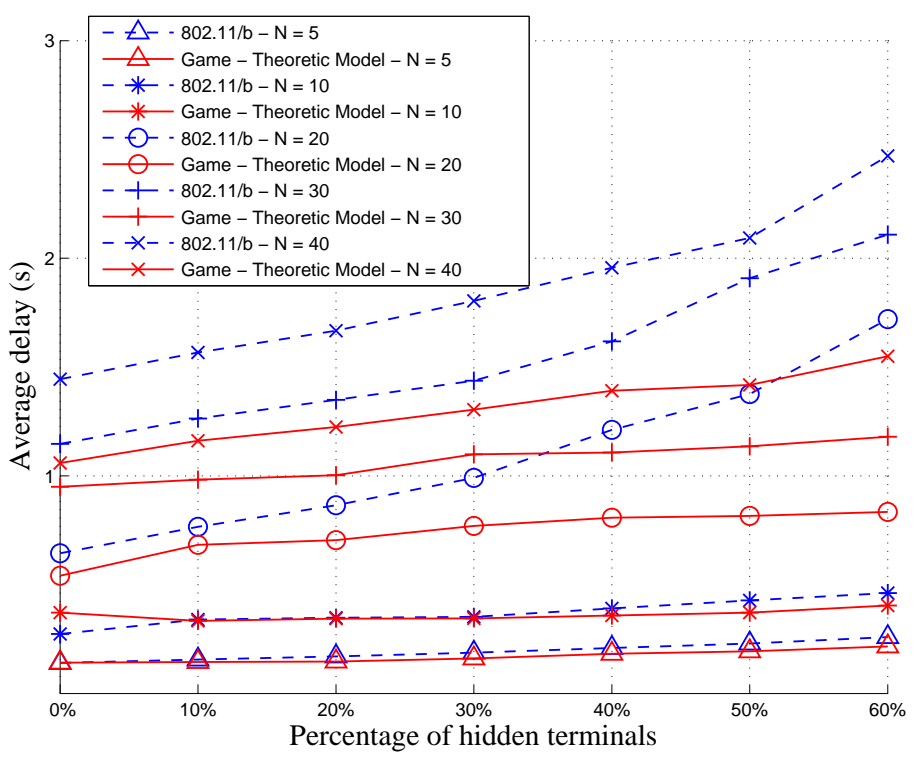

Figure 6.14: Average delay comparison for a network of 5-40 active nodes operating in RTS/CTS access (DCF \& game-theoretic design) method with 0-60 percent hidden terminal for each scenario 


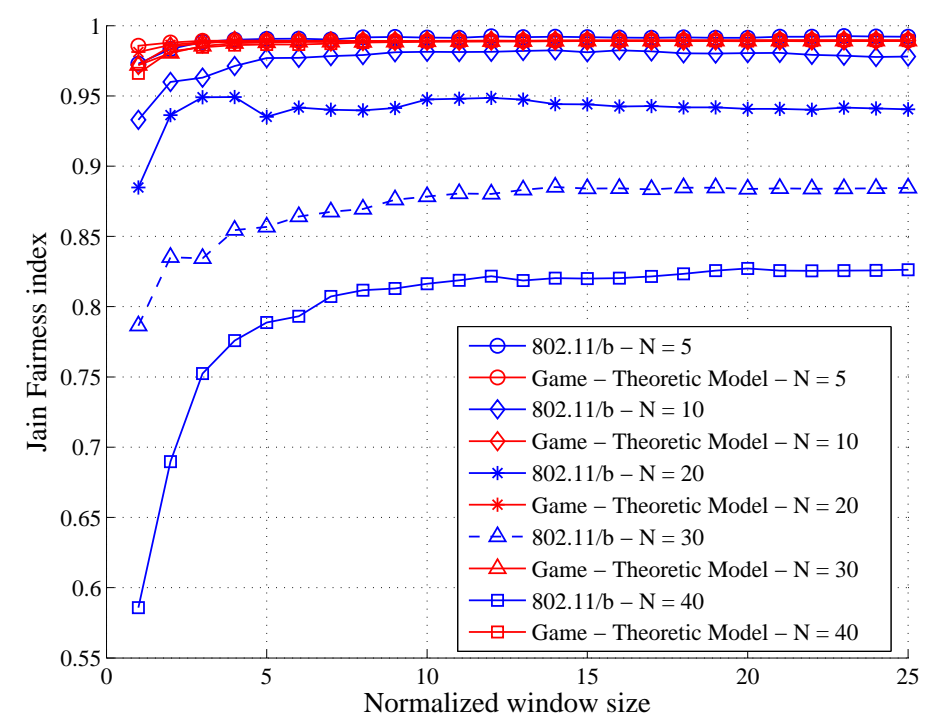

Figure 6.15: Fairness comparison for a network of 5-40 active nodes operating in basic access method.

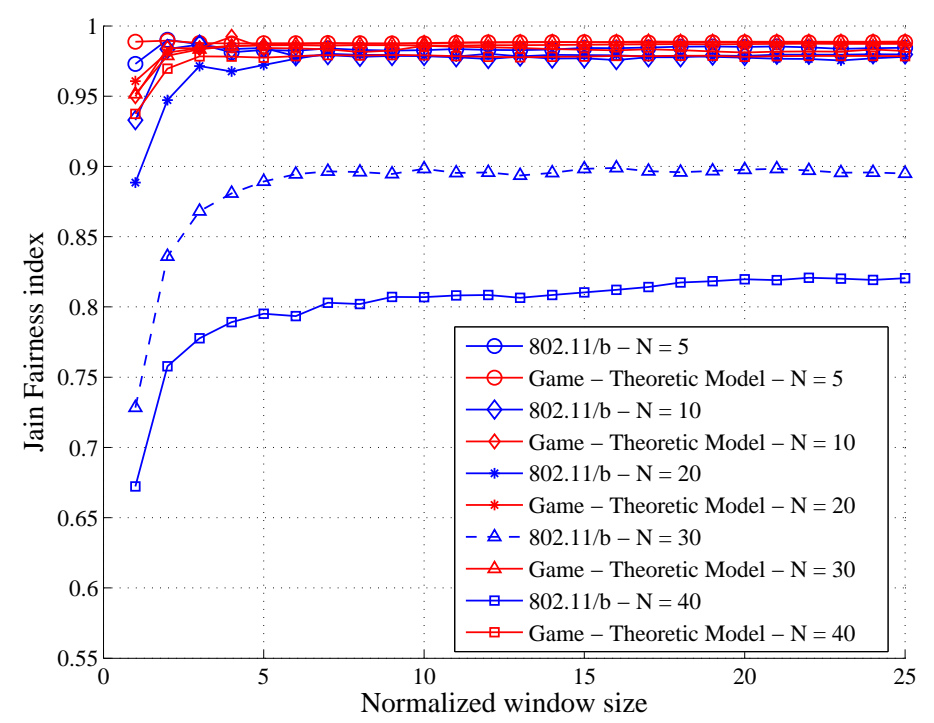

Figure 6.16: Fairness comparison for a network of 5-40 active nodes operating in RTS/CTS access method. 
$\triangle 802.11 / \mathrm{b}-$ hidden node $=0 \%$

$\triangle$ Game - Theoretic Model - hidden node $=0 \%$

$* 802.11 / \mathrm{b}-$ hidden node $=10 \%$

* Game - Theoretic Model - hidden node $=10 \%$

- $802.11 / \mathrm{b}-$ hidden node $=20 \%$

- Game - Theoretic Model - hidden node $=20 \%$

$+802.11 / \mathrm{b}-$ hidden node $=30 \%$

+ Game - Theoretic Model - hidden node $=30 \%$

$\times 802.11 / \mathrm{b}-$ hidden node $=40 \%$

$\star$ Game - Theoretic Model - hidden node $=40 \%$

$\diamond 802.11 / \mathrm{b}-$ hidden node $=50 \%$

$\diamond$ Game - Theoretic Model - hidden node $=50 \%$

- $802.11 / \mathrm{b}-$ hidden node $=60 \%$

- Game - Theoretic Model - hidden node $=60 \%$

(a) Legend

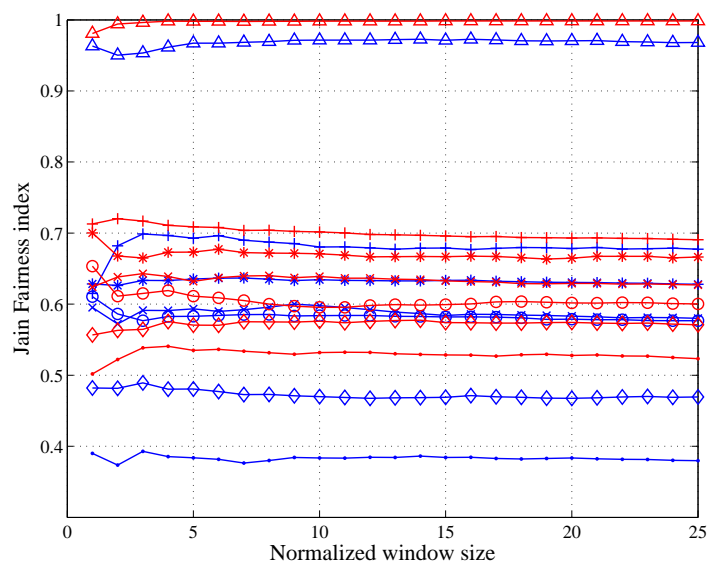

(c) $N=10$

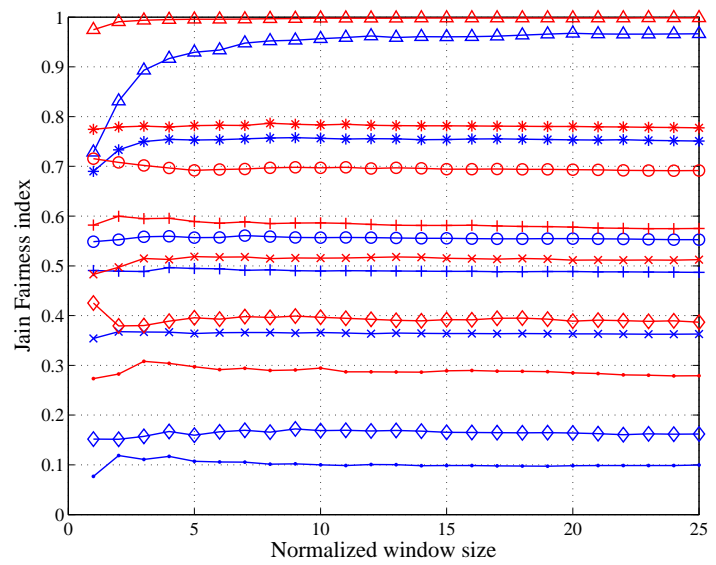

(e) $N=30$

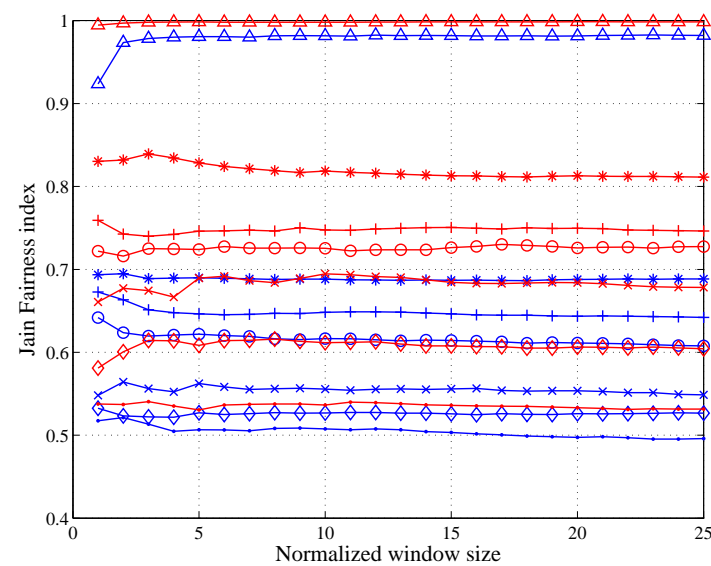

(b) $N=5$

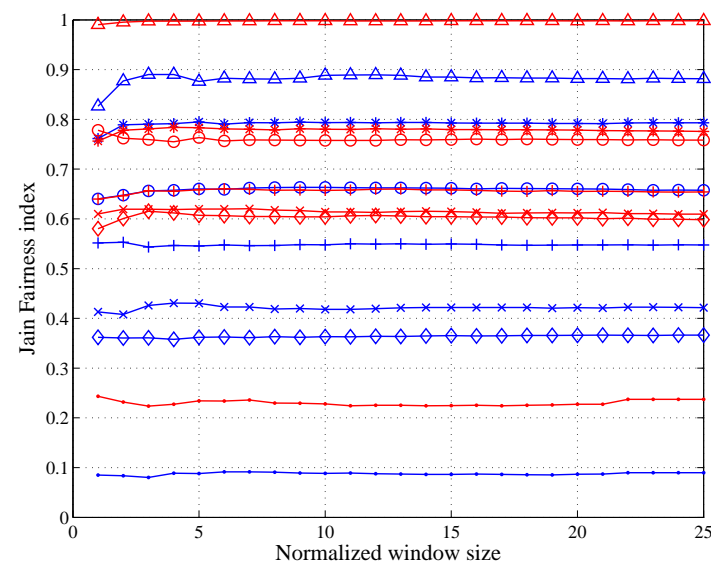

(d) $N=20$

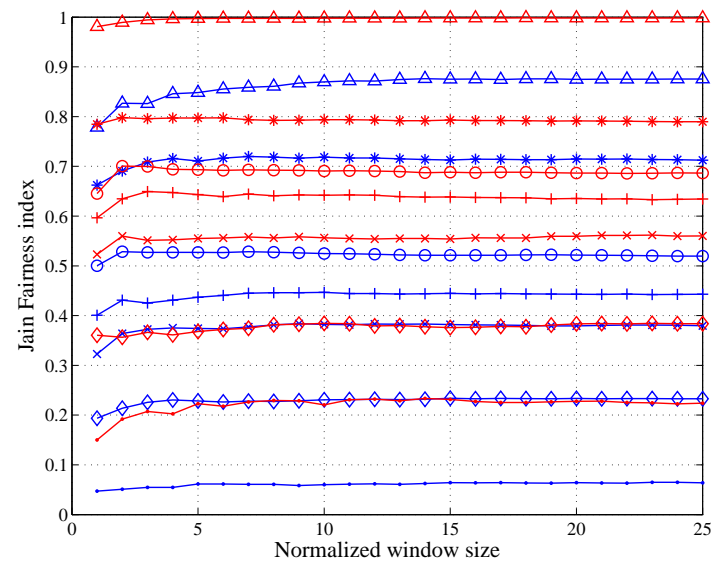

(f) $N=40$

Figure 6.17: Fairness comparison for a network of 5-40 active nodes operating in basic access method with 0-60 percent hidden terminal for each scenario. 
$\triangle 802.11 / \mathrm{b}$ - hidden node $=0 \%$

$\triangle$ Game - Theoretic Model - hidden node $=0 \%$

$* 802.11 / \mathrm{b}-$ hidden node $=10 \%$

* Game - Theoretic Model - hidden node $=10 \%$

- $802.11 / \mathrm{b}-$ hidden node $=20 \%$

- Game - Theoretic Model - hidden node $=20 \%$

$+802.11 / \mathrm{b}-$ hidden node $=30 \%$

- Game - Theoretic Model - hidden node $=30 \%$

$\star 802.11 / \mathrm{b}-$ hidden node $=40 \%$

$\star$ Game - Theoretic Model - hidden node $=40 \%$

$\diamond 802.11 / \mathrm{b}-$ hidden node $=50 \%$

$\checkmark$ Game - Theoretic Model - hidden node $=50 \%$

- $802.11 / \mathrm{b}-$ hidden node $=60 \%$

$\rightarrow$ Game - Theoretic Model - hidden node $=60 \%$

(a) Legend

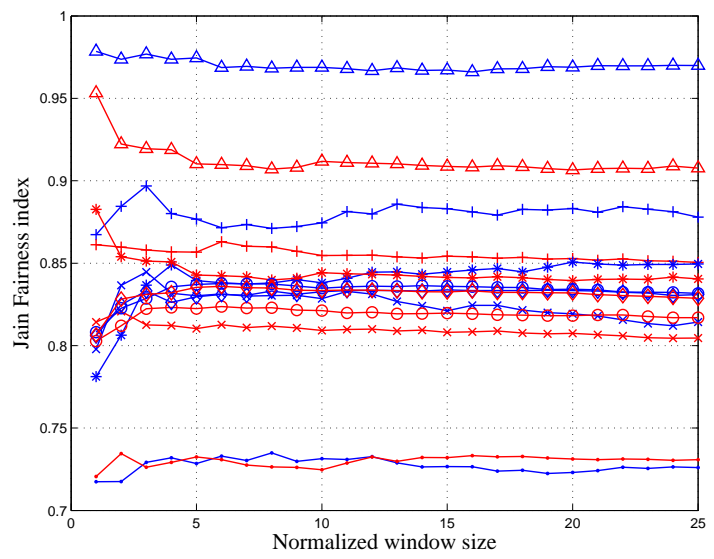

(c) $N=10$

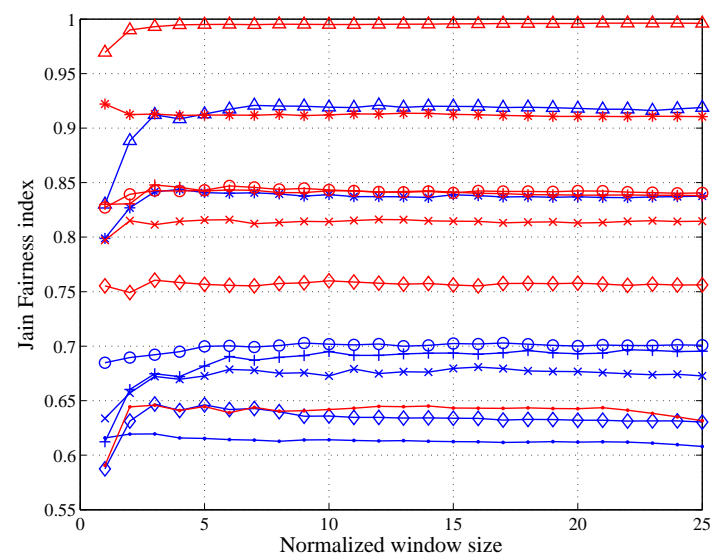

(e) $N=30$

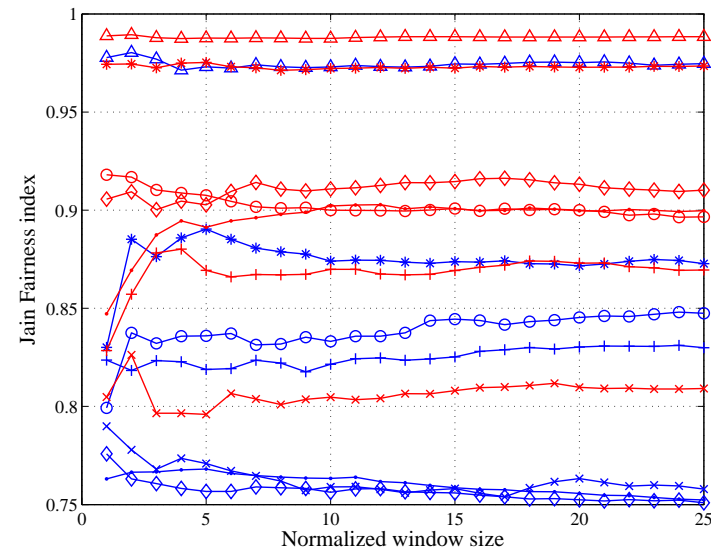

(b) $N=5$

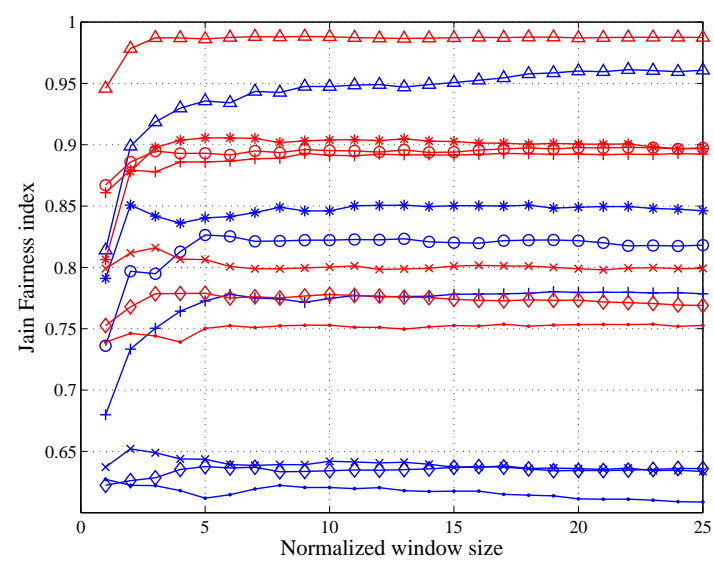

(d) $N=20$

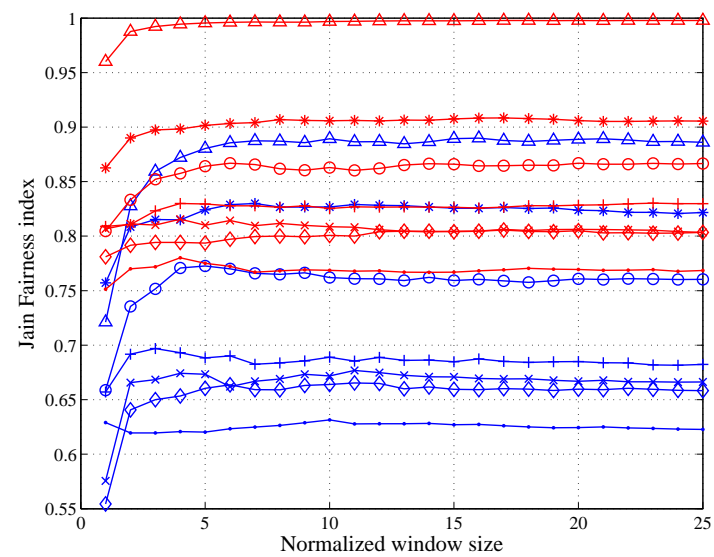

(f) $N=40$

Figure 6.18: Fairness comparison for a network of 5-40 active nodes operating in basic access method with 0-60 percent hidden terminal for each scenario. 


\section{CHAPTER 7}

\section{CONCLUSIONS}

This thesis proposes and explores a game-theoretic approach as a new paradigm for designing a distributed wireless MAC protocol. This study has focused on laying out a theoretical framework to design a reliable, robust, and efficient game-theoretic MAC protocol for wireless LANs operating under DCF, which is simple to implement and computationally efficient. We adopted a noncooperative, static, game-theoretic approach to model DCF in autonomous wireless networks where each node chooses its strategy (which is the channel access probability) to maximise its utility function.

Two games-theoritic model were proposed: The first game is designed for single-cell ad hoc networks [15] (see Chapter 3), and the second one is designed for centralised networks using DCF in the presence of hidden terminals [16] (see Chapter 4). Both games derive from CSMA/CA, in which the idle sense method is employed to design appropriate utility and payoff functions, as proposed by Heusse et al. [14]. The correct specification of a homogeneous utility function for single-cell games and per-node utility functions for centralised games in the presence of hidden terminals results in harmonized but independently determined performance objectives for all competing nodes.

Replacing the binary exponential backoff mechanism with an optimal contention window derived from idle sense method [14] preserves the independence of channel access probability from the conditional collision probability. Therefore, unlike DCF in the proposed 
access methods, wireless nodes can estimate the conditional collision probability by observing consecutive idle slots between transmissions. This means that contention control can be decoupled from the handling of failed transmissions. This results in a stable MAC protocol immune to a wide range of errors on the channel. Moreover, the design of the utility function based on optimal consecutive idle slots minimises the vulnerable period on the channel, which results in a lower number of collisions without the necessity to compromise the trade-off between throughput and channel access delay. As shown in numerical simulations (Chapter 6), the game-theoretic medium access control designs exhibit lower channel access delay, higher throughput, and significantly better short-term fairness (and ultimately better long-term fairness) than DCF. In addition to guiding medium access control design, the random access game model also provides an analytical framework to understand the equilibrium properties and dynamic properties of different medium access protocols.

In conclusion, it has been shown that employing a reasoned approach to design a distributed MAC protocol through the use of game theory in an "imperfect" wireless environment results in increased performance as observed through the wireless network metrics of throughput, channel access delay and fairness in a variety of network scenarios and topologies. Finally, this study demonstrates that game theory is an appropriate mathematical tool in dealing with today's wireless networks issues when it comes to resolving conflict situations over shared medium resources.

\subsection{Future Work}

A great deal of work is needed in order to elevate the proposed game-theoretic protocols from what is, so far, an encouraging and promising design framework to a complete and fully fledged medium access control protocol.

- Examining the coexistence of the proposed access methods and 802.11 DCF: It is 
important to examine the coexistence of all protocols together for the deployment of the proposed methods. It should be performed not only by simulations but also with the inclusion of real-world equipment in order to study the behaviour of their contention signals at the same time. One of the most appropriate methods for examining this coexistence in the real-life setting is to apply the proposed access methods in a testbed with the aim of assessing performance in comparison with the 802.11 DCF for a variety of radio channel environments. Most importantly, this will facilitate the research of the robust estimation of conditional collision probability and the analysis of the setting of various control parameters that determine the dynamic properties of the proposed access methods.

- Extending the proposed access methods to multi-cell network: There is a need to investigate other contention resolution algorithms that have the ability to deal with the issue of exposed terminals, particularly in DCF with centralised architecture. The reason is that in DCF, a node in range of two other nodes will reduce its channel access probability by increasing its contention window size, which results in less frequent channel access when compared to its neighbours. Network performance degradation are then witnessed, particularly in terms of fairness. This issue can be further exasperated if the neighbourhood nodes do not successfully communicate, therefore they might successfully and simultaneously transmit. Such a situation means that the node present in the overlapping area between cells will see that the channel is idle less frequently, meaning a decreased propensity to achieve channel access. Notably, all protocols based on CSMA/CA experience this problem, with the current solution to this challenge is based on the proper configuration of APs so as to ensure cells do not overlap or function on orthogonal channels.

- Extending the proposed access methods to the network where wireless nodes operate 
in different classes of traffic. In this study, it is assumed that nodes in the network are homogeneous and belong to same QoS class. However, in reality, wireless nodes operate using different bit rates in the network. Accordingly, there is a need to provide a general analytical framework with the ability to model a large class of system-wide quality-of-service (QoS) models through the criteria of per-node utility function; this would help to ensure high channel utilisation and time fairness in a wireless network with bit rate diversity.

- It is also worth investigating different utility function designs. There are a number of different functions that may be used so as to symbolise players' utility function in the proposed access methods. These utility functions are mostly function of channel access probability. In order to design an appropriate utility functions that can converge to desirable equilibrium, the design should be based on reverse engineering from existing channel access methods or forward engineering from desired operating points (e.g., [15, 16]) - all of which merit in-depth investigation. These functions can be compared in terms of network performance through mathematical network models, and also, based on effectiveness in real-world environment through simulations and testbed examinations.

- It is also worth investigating different equilibrium concepts. Notably, this proposal considers only the Nash equilibrium as the solution; nevertheless, such solution concept may be insufficient in delivering optimum network performance. In order to ensure Nash equilibrium superiority, a number of other related concepts should be examined. For instance, the Nash bargaining solution-which is Paretooptimal - should be taken into account in regard to fairness and efficiency. An alternative solution is correlated equilibrium. This solution concept is more generalised when contrasted alongside Nash equilibrium, and is also recognised as incurring less 
computational complexity throughout the course of determination.

- The creation and development of application-centric game models for distributed wireless protocol: Conventional random channel access schemes might not be applicable in a number of specific wireless network scenarios, including wireless sensor networks and vehicular networks. In order to design a MAC protocols for particular applications, there are two steps that are need to be taken: primarily, application criteria and the resource constraints must be specified. Secondly, all restrictions caused by the specified requirements in the first step need to be taken into account during the design process. For example, transmission delay (i.e., emergency messaging delay) is an important application criteria in vehicular networks. In such networks, high mobility is also one of the application criteria (high mobility rapidly changes the topology of the vehicular network). Limited bandwidth is one of the resource constraints. Variable vehicle density is also one of the resource constraints. To design a comprehensive utility functions for such networks, all associated parameters and restrictions need to be considered. Because of specifications and requirements of the applications, the development of game models for application-centric random channel access is recognised as being far more problematic than general wireless protocols like wireless LANs.

- As DCF is expected to continue to play a prominent role in 5th generation mobile communication technologies to address the issue of high bandwidth demand at base stations, the modelling random channel access and channel allocation problem in a heterogeneous wireless access network using game theory is highly promising solution to address the issues regarding the bandwidth demand. In the specific context of a heterogeneous network, service providers for the various wireless access networks - in addition to mobile users - might compete or cooperate with one another. 
Game-theoretic modelling and the examination of any and all interactions across mobile nodes and service providers is an interesting research topic. In this same regard, in a heterogeneous network where a bi-level hierarchy exists, the rate control and transmission power issue for random channel access may be modelled with the application of game theory, and an overall optimal random channel access protocol can be designed. 


\section{LIST OF REFERENCES}

[1] G.L. Stüber. Principles of Mobile Communication. Springer, 2011.

[2] Ajay Chandra V. Gummalla and John O. Limb. Wireless medium access control protocols. Communications Surveys Tutorials, IEEE, 3(2):2-15, Second 2000.

[3] A. Goldsmith. Wireless Communications. Cambridge University Press.

[4] Jianbo Gao and Izhak Rubin. Analysis of random access protocol under bursty traffic. In EhabS. Al-Shaer and Giovanni Pacifici, editors, Management of Multimedia on the Internet, volume 2216 of Lecture Notes in Computer Science, pages 71-84. Springer Berlin Heidelberg, 2001.

[5] Norman Abramson. Development of the alohanet. Information Theory, IEEE Transactions on, 31(2):119-123, 1985.

[6] Lawrence G. Roberts. Extensions of packet communication technology to a hand held personal terminal. In Proceedings of the May 16-18, 1972, Spring Joint Computer Conference, AFIPS '72 (Spring), pages 295-298, New York, NY, USA, 1972. ACM.

[7] Phil Karn. MACA - a new channel access method for packet radio. In $A R R L / C R R L$ Amateur Radio 9th Computer Networking Conference, pages 134-140, September 1990.

[8] Vaduvur Bharghavan, Alan Demers, Scott Shenker, and Lixia Zhang. Macaw: A media access protocol for wireless lan's. SIGCOMM Comput. Commun. Rev., 24(4):212225, October 1994.

[9] G. Bianchi. Performance analysis of the ieee 802.11 distributed coordination function. Selected Areas in Communications, IEEE Journal on, 18(3):535-547, 2000.

[10] Hui Ma, Xing Li, Hewu Li, Peiyun Zhang, Shixin Luo, and Cong Yuan. Dynamic optimization of ieee $802.11 \mathrm{csma} / \mathrm{ca}$ based on the number of competing stations. In Communications, 2004 IEEE International Conference on, volume 1, pages 191-195, 2004 .

[11] A.B. MacKenzie, L. DaSilva, and L.A. DaSilva. Game Theory for Wireless Engineers. Synthesis lectures on communications. Morgan \& Claypool Publishers, 2006. 
[12] K. Akkarajitsakul, E. Hossain, D. Niyato, and Dong In Kim. Game theoretic approaches for multiple access in wireless networks: A survey. Communications Surveys Tutorials, IEEE, 13(3):372-395, Third 2011.

[13] Drew Fudenberg and Jean Tirole. Game Theory. MIT Press, Cambridge, MA, 1991.

[14] Martin Heusse, Franck Rousseau, Romaric Guillier, and Andrzej Duda. Idle sense: an optimal access method for high throughput and fairness in rate diverse wireless lans. In Proceedings of the 2005 conference on Applications, technologies, architectures, and protocols for computer communications, SIGCOMM '05, pages 121-132, New York, NY, USA, 2005. ACM.

[15] S.H.E.M. Najafabadi and C.C. Constantinou. Game theoretic approach to medium access control in wireless networks. In Wireless Communications and Networking Conference (WCNC), 2013 IEEE, pages 872-877, April 2013.

[16] S.H.E.M. Najafabadi and C.C. Constantinou. A game theoretic model for wireless medium access control in the presence of hidden terminals. In 2013 IEEE 24th International Symposium on Personal, Indoor and Mobile Radio Communications (PIMRC) 2013, pages 1741-1746, London, United Kingdom, September 2013.

[17] Wireless lan medium access control (mac) and physical layer (phy) specifications. IEEE Std 802.11, 1999.

[18] Sachin Katti, Shyamnath Gollakota, and Dina Katabi. Embracing wireless interference: Analog network coding. In Proceedings of the 2007 Conference on Applications, Technologies, Architectures, and Protocols for Computer Communications, SIGCOMM '07, pages 397-408, New York, NY, USA, 2007. ACM.

[19] Shyamnath Gollakota and Dina Katabi. Zigzag decoding: Combating hidden terminals in wireless networks. In Proceedings of the ACM SIGCOMM 2008 Conference on Data Communication, SIGCOMM '08, pages 159-170, New York, NY, USA, 2008. ACM.

[20] Daniel Halperin, Thomas Anderson, and David Wetherall. Taking the sting out of carrier sense: Interference cancellation for wireless lans. In Proceedings of the 14th ACM International Conference on Mobile Computing and Networking, MobiCom '08, pages 339-350, New York, NY, USA, 2008. ACM.

[21] B. Radunovic, D. Gunawardena, P. Key, A. Proutiere, N. Singh, V. Balan, and G. DeJean. Rethinking indoor wireless mesh design: Low power, low frequency, fullduplex. In Wireless Mesh Networks (WIMESH 2010), 2010 Fifth IEEE Workshop on, pages 1-6, June 2010.

[22] S. Sen, R.R. Choudhury, and S. Nelakuditi. Csma/cn: Carrier sense multiple access with collision notification. Networking, IEEE/ACM Transactions on, 20(2):544-556, April 2012. 
[23] 1.L. Gheorma and G.K. Gopalakrishnan. Rf photonic techniques for same frequency simultaneous duplex antenna operation. Photonics Technology Letters, IEEE, 19(13):1014-1016, July 2007.

[24] D.W. Bliss, P.A. Parker, and A.R. Margetts. Simultaneous transmission and reception for improved wireless network performance. In Statistical Signal Processing, 2007. SSP '07. IEEE/SP 14th Workshop on, pages 478-482, Aug 2007.

[25] Allen Miu, Godfrey Tan, Hari Balakrishnan, and John Apostolopoulos. Divert: Finegrained path selection for wireless lans. In Proceedings of the $2 N d$ International Conference on Mobile Systems, Applications, and Services, MobiSys '04, pages 203216, New York, NY, USA, 2004. ACM.

[26] Daniel Aguayo, John Bicket, Sanjit Biswas, Glenn Judd, and Robert Morris. Linklevel measurements from an 802.11b mesh network. In Proceedings of the 2004 Conference on Applications, Technologies, Architectures, and Protocols for Computer Communications, SIGCOMM '04, pages 121-132, New York, NY, USA, 2004. ACM.

[27] David Kotz, Calvin Newport, Robert S. Gray, Jason Liu, Yougu Yuan, and Chip Elliott. Experimental evaluation of wireless simulation assumptions. In Proceedings of the 7th ACM International Symposium on Modeling, Analysis and Simulation of Wireless and Mobile Systems, MSWiM '04, pages 78-82, New York, NY, USA, 2004. ACM.

[28] Godfrey Tan and John Guttag. Time-based fairness improves performance in multirate wlans. In Proceedings of the Annual Conference on USENIX Annual Technical Conference, ATEC '04, pages 23-23, Berkeley, CA, USA, 2004. USENIX Association.

[29] A. Kochut, A. Vasan, A.U. Shankar, and A. Agrawala. Sniffing out the correct physical layer capture model in 802.11b. In Network Protocols, 2004. ICNP 2004. Proceedings of the 12th IEEE International Conference on, pages 252-261, Oct 2004.

[30] F.A. Tobagi and L. Kleinrock. Packet switching in radio channels: Part ii-the hidden terminal problem in carrier sense multiple-access and the busy-tone solution. Communications, IEEE Transactions on, 23(12):1417-1433, Dec 1975.

[31] Kai-Chuang Huang and Kwang-Cheng Chen. Interference analysis of nonpersistent csma with hidden terminals in multicell wireless data networks. In Personal, Indoor and Mobile Radio Communications, 1995. PIMRC'95. Wireless: Merging onto the Information Superhighway., Sixth IEEE International Symposium on, volume 2, pages 907-911 vol.2, Sep 1995.

[32] Saikat Ray, David Starobinski, and Jeffrey B. Carruthers. Performance of wireless networks with hidden nodes: A queuing-theoretic analysis. Comput. Commun., 28(10):1179-1192, June 2005. 
[33] Harshal S. Chhaya and Sanjay Gupta. Performance modeling of asynchronous data transfer methods of ieee 802.11 mac protocol. Wirel. Netw., 3(3):217-234, August 1997.

[34] Yu Wang and J.J. Garcia-Luna-Aceves. Performance of collision avoidance protocols in single-channel ad hoc networks. In Network Protocols, 2002. Proceedings. 10th IEEE International Conference on, pages 68-77, Nov 2002.

[35] Haitao Wu, Yong Peng, Keping Long, Shiduan Cheng, and Jian Ma. Performance of reliable transport protocol over ieee 802.11 wireless lan: analysis and enhancement. In INFOCOM 2002. Twenty-First Annual Joint Conference of the IEEE Computer and Communications Societies. Proceedings. IEEE, volume 2, pages 599-607 vol.2, 2002 .

[36] Eustathia Ziouva and Theodore Antonakopoulos. Csma/ca performance under high traffic conditions: Throughput and delay analysis. Comput. Commun., 25(3):313321, February 2002.

[37] K. Sakakibara, S. Chikada, and J. Yamakita. Analysis of unsaturation throughput of ieee 802.11 def. In Personal Wireless Communications, 2005. ICPWC 2005. 2005 IEEE International Conference on, pages 134-138, Jan 2005.

[38] K. Duffy, D. Malone, and D.J. Leith. Modeling the 802.11 distributed coordination function in non-saturated conditions. Communications Letters, IEEE, 9(8):715-717, Aug 2005.

[39] Fu-Yi Hung and Ivan Marsic. Performance analysis of the ieee $802.11 \mathrm{dcf}$ in the presence of the hidden stations. Computer Networks, 54(15):2674 - 2687, 2010.

[40] Taejoon Kim and Jong-Tae Lim. Throughput analysis considering coupling effect in ieee 802.11 networks with hidden stations. Communications Letters, IEEE, 13(3):175-177, March 2009.

[41] Youngjip Kim and Chong-Ho Choi. Analysis of the ieee 802.11 back-off mechanism in presence of hidden nodes. IEICE Transactions, 92-B(4):1291-1299, 2009.

[42] O. Ekici and A. Yongacoglu. Ieee 802.11a throughput performance with hidden nodes. Communications Letters, IEEE, 12(6):465-467, June 2008.

[43] Athanasia Tsertou, David I. Laurenson, and John S. Thompson. A new approach for the throughput analysis of ieee 802.11 in networks with hidden terminals, 2005.

[44] Haitao Wu, Fan Zhu, Qian Zhang, and Zhisheng Niu. Wsn02-1: Analysis of ieee 802.11 dcf with hidden terminals. In Global Telecommunications Conference, 2006. GLOBECOM '06. IEEE, pages 1-5, Nov 2006. 
[45] Fu-Yi Hung, S. Pai, and I. Marsic. Performance modeling and analysis of the ieee 802.11 distribution coordination function in presence of hidden stations. In Military Communications Conference, 2006. MILCOM 2006. IEEE, pages 1-7, Oct 2006.

[46] A. Tsertou and D.I. Laurenson. Revisiting the hidden terminal problem in a csma/ca wireless network. Mobile Computing, IEEE Transactions on, 7(7):817-831, 2008.

[47] F. Cali, M. Conti, and Enrico Gregori. Dynamic tuning of the ieee 802.11 protocol to achieve a theoretical throughput limit. Networking, IEEE/ACM Transactions on, 8(6):785-799, 2000.

[48] L. Gannoune and S. Robert. Dynamic tuning of the contention window minimum (cwmin) for enhanced service differentiation in ieee 802.11 wireless ad-hoc networks. 1:311 - 317 Vol.1, sept. 2004.

[49] G. Bianchi and I. Tinnirello. Kalman filter estimation of the number of competing terminals in an ieee 802.11 network. In INFOCOM 2003. Twenty-Second Annual Joint Conference of the IEEE Computer and Communications. IEEE Societies, volume 2, pages 844-852 vol.2, March 2003.

[50] G. Bianchi, L. Fratta, and M. Oliveri. Performance evaluation and enhancement of the csma/ca mac protocol for 802.11 wireless lans. In Personal, Indoor and Mobile Radio Communications, 1996. PIMRC'96., Seventh IEEE International Symposium on, volume 2, pages 392-396 vol.2, Oct 1996.

[51] L. Bononi, M. Conti, and Enrico Gregori. Runtime optimization of ieee 802.11 wireless lans performance. Parallel and Distributed Systems, IEEE Transactions on, 15(1):66-80, Jan 2004.

[52] Qiang Ni, I. Aad, C. Barakat, and T. Turletti. Modeling and analysis of slow cw decrease ieee 802.11 wlan. In Personal, Indoor and Mobile Radio Communications, 2003. PIMRC 2003. 14th IEEE Proceedings on, volume 2, pages 1717-1721 vol.2, Sept 2003.

[53] Younggoo Kwon, Yuguang Fang, and H. Latchman. A novel mac protocol with fast collision resolution for wireless lans. In INFOCOM 2003. Twenty-Second Annual Joint Conference of the IEEE Computer and Communications. IEEE Societies, volume 2, pages 853-862 vol.2, March 2003.

[54] Tiantong You, Chi-Hsiang Yeh, and H. Hassanein. A new class of collision prevention mac protocols for wireless ad hoc networks. In Communications, 2003. ICC '03. IEEE International Conference on, volume 2, pages 1135-1140 vol.2, May 2003.

[55] Thyagarajan Nandagopal, Tae-Eun Kim, Xia Gao, and Vaduvur Bharghavan. Achieving mac layer fairness in wireless packet networks. In Proceedings of the 6th Annual International Conference on Mobile Computing and Networking, MobiCom '00, pages 87-98, New York, NY, USA, 2000. ACM. 
[56] Nah-Oak Song, Byung-Jae Kwak, Jabin Song, and M.E. Miller. Enhancement of ieee 802.11 distributed coordination function with exponential increase exponential decrease backoff algorithm. In Vehicular Technology Conference, 2003. VTC 2003Spring. The 57th IEEE Semiannual, volume 4, pages 2775-2778 vol.4, April 2003.

[57] Chunyu Hu and J.C. Hou. A novel approach to contention control in ieee 802.11eoperated wlans. In INFOCOM 200\%. 26th IEEE International Conference on Computer Communications. IEEE, pages 1190-1198, May 2007.

[58] Robert J. Aumann. Subjectivity and correlation in randomized strategies. Journal of Mathematical Economics, 1(1):67-96, March 1974.

[59] Klaus Ritzberger. Foundations of non-cooperative game theory. Oxford Univ. Press, Oxford [u.a.], 1. publ edition, 2002.

[60] Michael L. Littman. Markov games as a framework for multi-agent reinforcement learning. In IN PROCEEDINGS OF THE ELEVENTH INTERNATIONAL CONFERENCE ON MACHINE LEARNING, pages 157-163. Morgan Kaufmann, 1994.

[61] Nikos Vlassis. A concise introduction to multiagent systems and distributed ai, 2003.

[62] Richard S. Sutton and Andrew G. Barto. Reinforcement Learning: An Introduction. MIT Press, 1998.

[63] D. Chatterjee and T.F. Wong. Resource allocation and cooperative behavior in fading multiple-access channels under uncertainty. In Military Communications Conference, 2009. MILCOM 2009. IEEE, pages 1-7, Oct 2009.

[64] B. Shrestha, D. Niyato, Zhu Han, and E. Hossain. Wireless access in vehicular environments using bittorrent and bargaining. In Global Telecommunications Conference, 2008. IEEE GLOBECOM 2008. IEEE, pages 1-5, Nov 2008.

[65] Levente Buttyan and Jean-Pierre Hubaux. Security and Cooperation in Wireless Networks: Thwarting Malicious and Selfish Behavior in the Age of Ubiquitous Computing. Cambridge University Press, New York, NY, USA, 2007.

[66] H. Inaltekin and S. Wicker. The analysis of a game theoretic mac protocol for wireless networks. In Sensor and Ad Hoc Communications and Networks, 2006. SECON '06. 2006 3rd Annual IEEE Communications Society on, volume 1, pages 296-305, Sept 2006.

[67] Younggeun Cho and F.A. Tobagi. Cooperative and non-cooperative aloha games with channel capture. In Global Telecommunications Conference, 2008. IEEE GLOBECOM 2008. IEEE, pages 1-6, Nov 2008.

[68] Younggeun Cho, Chan-Soo Hwang, and F.A. Tobagi. Design of robust random access protocols for wireless networks using game theoretic models. In INFOCOM 2008. The 27th Conference on Computer Communications. IEEE, pages -, April 2008. 
[69] HyungJune Lee, Hyukjoon Kwon, A. Motskin, and L. Guibas. Interference-aware mac protocol for wireless networks by a game-theoretic approach. In INFOCOM 2009, IEEE, pages 1854-1862, April 2009.

[70] Tao Cui, Lijun Chen, and S.H. Low. A game-theoretic framework for medium access control. Selected Areas in Communications, IEEE Journal on, 26(7):1116-1127, September 2008.

[71] Y.E. Sagduyu, R. Berry, and Anthony Ephremides. Mac games for distributed wireless network security with incomplete information of selfish and malicious user types. In Game Theory for Networks, 2009. GameNets '09. International Conference on, pages 130-139, May 2009.

[72] Y.E. Sagduyu and Anthony Ephremides. Power control and rate adaptation as stochastic games for random access. In Decision and Control, 2003. Proceedings. 42nd IEEE Conference on, volume 4, pages 4202-4207 vol.4, Dec 2003.

[73] P. Nuggehalli, M. Sarkar, K. Kulkarni, and R.R. Rao. A game-theoretic analysis of qos in wireless mac. In INFOCOM 2008. The 27th Conference on Computer Communications. IEEE, pages -, April 2008.

[74] Dandan Wang, Cristina Comaniciu, H. Minn, and N. Al-Dhahir. A game-theoretic approach for exploiting multiuser diversity in cooperative slotted aloha. Wireless Communications, IEEE Transactions on, 7(11):4215-4225, November 2008.

[75] R.T.B. Ma, V. Misra, and D. Rubenstein. An analysis of generalized slotted-aloha protocols. Networking, IEEE/ACM Transactions on, 17(3):936-949, June 2009.

[76] Guopeng Zhang, Hailin Zhang, and Liqiang Zhao. A novel mac scheme for wireless lans from the perspective of game theory. In Wireless, Mobile and Sensor Networks, 200\%. (CCWMSN07). IET Conference on, pages 112-116, Dec 2007.

[77] S. Chowdhury, S. Dutta, K. Mitra, D.K. Sanyal, M. Chattopadhyay, and S. Chattopadhyay. Game-theoretic modeling and optimization of contention-prone medium access phase in ieee 802.16/wimax networks. In Broadband Communications, Information Technology Biomedical Applications, 2008 Third International Conference on, pages 335-342, Nov 2008.

[78] Lijun Chen, S.H. Low, and J.C. Doyle. Contention control: A game-theoretic approach. In Decision and Control, 2007 46th IEEE Conference on, pages 3428-3434, Dec 2007.

[79] M. Felegyhazi, M. Cagalj, and J-P Hubaux. Efficient mac in cognitive radio systems: A game-theoretic approach. Wireless Communications, IEEE Transactions on, 8(4):1984-1995, April 2009. 
[80] Liqiang Zhao, Jie Zhang, and Hailin Zhang. Using incompletely cooperative game theory in wireless mesh networks. Network, IEEE, 22(1):39-44, Jan 2008.

[81] J. Konorski. A game-theoretic study of csma/ca under a backoff attack. Networking, IEEE/ACM Transactions on, 14(6):1167-1178, Dec 2006.

[82] J. E. Marsden R. Abraham and T. Ratiu. Manifolds, Tensor Analysis, and Applications. Springer-Verlag, second edition edition, 1988.

[83] D.P. Bertsekas and R.G. Gallager. Data networks. Prentice-Hall international editions. Prentice Hall, 1992.

[84] Walid Saad Zhu Han, Dusit Niyato and Tamer Basar. Game Theory in Wireless and Communication Networks: Theory, Models, and Applications. Cambridge University Press, Cambridge/GB, 2012.

[85] OMNeT ++ Community. OMNeT ++ modeler website. url: http://www.omnetpp.org/. page accessed on february 27, 2013.

[86] Stuart Kurkowski, Tracy Camp, and Michael Colagrosso. Manet simulation studies: The incredibles. SIGMOBILE Mob. Comput. Commun. Rev., 9(4):50-61, October 2005 .

[87] ns2 (2010) the network simulator - ns-2. url:http://www.isi.edu/nsnam/ns/. page accessed on february 27, 2013.

[88] Opnet technologies inc (2010) opnet modeler. url:http://www.opnet.com/solutions/ network_rd/modeler.html. page accessed on february 27, 2013.

[89] Y. Yuan J. Liu, L. F. Perrone and D. Nicol. The simulator for wireless ad hoc networks (swan). url: http://www.eg.bucknell.edu/swan/. page accessed on february 27, 2013.

[90] The qualnetô communications simulation platform (qualnet). url:http://web.scalable-networks.com/content/qualnet/. page accessed on february 27, 2013.

[91] OMNeT++-MiXiM modeling framework for mobile and fixed wireless networks. url: http://mixim.sourceforge.net/. page accessed on february 27, 2013.

[92] Jerry Banks, John S. Carson, Barry L. Nelson, and David M. Nicol. Discrete-Event System Simulation (5th Edition). Prentice Hall, 5 edition, July 2009.

[93] Robert G. Sargent. Verification and validation of simulation models. In Proceedings of the 3rth conference on Winter simulation, WSC '05, pages 130-143. Winter Simulation Conference, 2005. 
[94] Dimitri Bertsekas and Robert Gallager. Data networks. Prentice-Hall, Inc., Upper Saddle River, NJ, USA, 1987.

[95] Harshal S. Chhaya and Sanjay Gupta. Performance modeling of asynchronous data transfer methods of IEEE 802.11 MAC protocol. Wirel. Netw., 3(3):217-234, 1997.

[96] Tien-Shin Ho and Kwang-Cheng Chen. Performance analysis of IEEE 802.11 CSMA/CA medium access control protocol. Personal, Indoor and Mobile Radio Communications, 1996. PIMRC'96., Seventh IEEE International Symposium on, 2:407411 vol.2, October 1996.

[97] Rajendra K. Jain, Dah-Ming W. Chiu, and William R. Hawe. A quantitative measure of fairness and discrimination for resource allocation in shared computer systems. September 1984. 


\section{APPENDIX A DERIVATION OF UTILITY FUNCTIONS}

\section{A.1 Derevation of Equation $3.2 .19 \& 3.2 .22$}

In $\$ 3.2 .3$, we use the fact that $U_{i}^{\prime}\left(P_{i}^{\tau *}\right)=\mathcal{C}_{i}\left(\mathbf{P}^{\tau *}\right)$ at the nontrivial Nash equilibrium. By Equation 3.2.5 we have:

$$
\begin{aligned}
& 1-\left(1-P_{i}^{\tau}\right)^{N-1}=U_{i}^{\prime}\left(P_{i}^{\tau}\right) \\
& \left(1-P_{i}^{\tau}\right)^{N-1}=1-U_{i}^{\prime}\left(P_{i}^{\tau}\right) \\
& \left(1-P_{i}^{\tau}\right)^{N}=\left(1-P_{i}^{\tau}\right)\left(1-U_{i}^{\prime}\left(P_{i}^{\tau}\right)\right),
\end{aligned}
$$

then based on 3.2 .13 based on 3.2.21 we have :

$$
P_{i}^{i d l e}\left(\mathbf{P}^{\tau}\right)=\left(1-P_{i}^{\tau}\right)\left(1-U_{i}^{\prime}\left(P_{i}^{\tau}\right)\right), \forall i \in N
$$

in $\$ 3.2 .4$ we also have $P_{i}^{\text {idle }}\left(\mathbf{P}^{\tau}\right)=e^{-\xi}\left(1+P_{i}^{\tau}\right)$ therefore Equation A.1.2 becomes:

$$
\begin{aligned}
& \left(1-P_{i}^{\tau}\right)\left(1-U_{i}^{\prime}\left(P_{i}^{\tau}\right)\right)=e^{-\xi}\left(1+P_{i}^{\tau}\right) \\
& 1-U_{i}^{\prime}\left(P_{i}^{\tau}\right)=\frac{e^{-\xi}\left(1+P_{i}^{\tau}\right)}{\left(1-P_{i}^{\tau}\right)} \\
& U_{i}^{\prime}\left(P_{i}^{\tau}\right)=1-\frac{e^{-\xi}\left(1+P_{i}^{\tau}\right)}{\left(1-P_{i}^{\tau}\right)} \\
& U_{i}\left(P_{i}^{\tau}\right)=\int 1-\frac{e^{-\xi}\left(1+P_{i}^{\tau}\right)}{\left(1-P_{i}^{\tau}\right)} d P_{i}^{\tau} \\
& U_{i}\left(P_{i}^{\tau}\right)=\left(1+e^{-\xi}\right) P_{i}^{\tau}+2 e^{-\xi} \ln \left(1-P_{i}^{\tau}\right) .
\end{aligned}
$$

Equation 4.2.22 \& 4.2.24 can be derived exactly in the same manner. 


\section{APPENDIX B NORMALISED DELAY DISTRIBUTION}

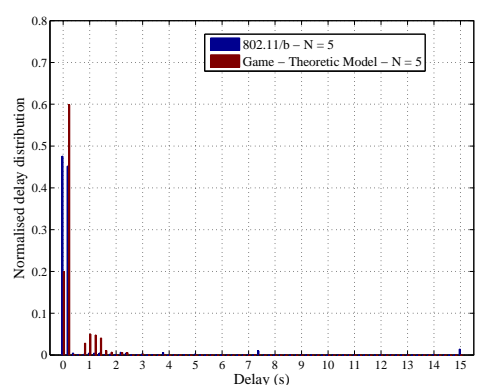

(a) Linear scale

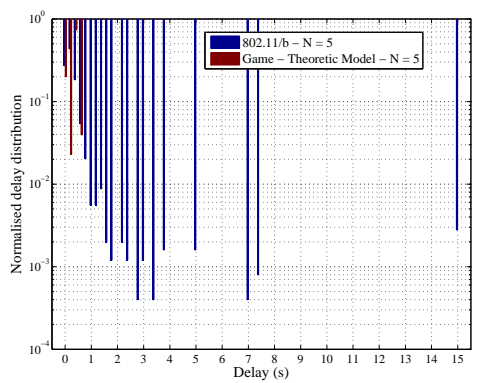

(d) Logarithmic scale

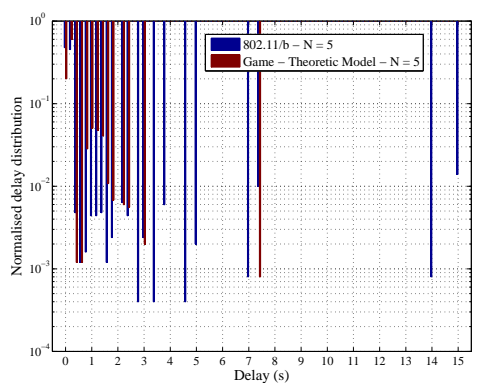

(b) Logarithmic scale

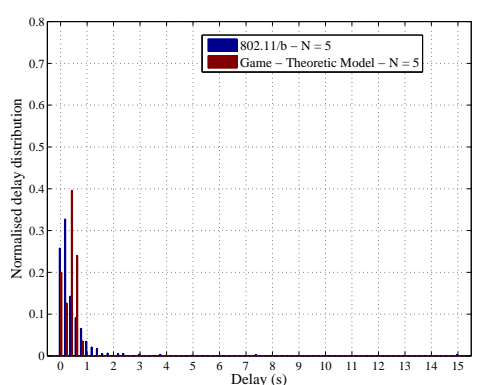

(e) Linear scale

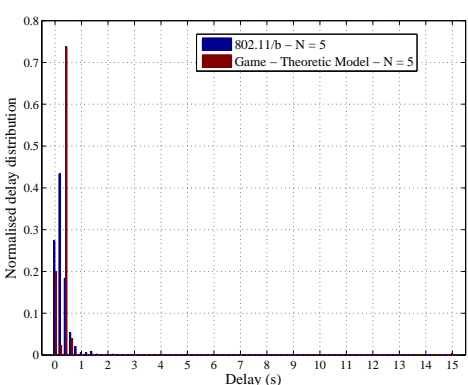

(c) Linear scale

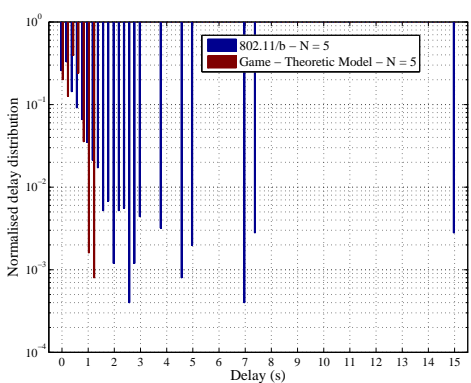

(f) Logarithmic scale

Figure B.1: Normalised delay distribution comparison for a network of 5 active nodes, operating in basic access method (DCF \& game-theoretic design) with 10-30 percent hidden terminal for each scenario. The bin-size is 0.2 seconds. 


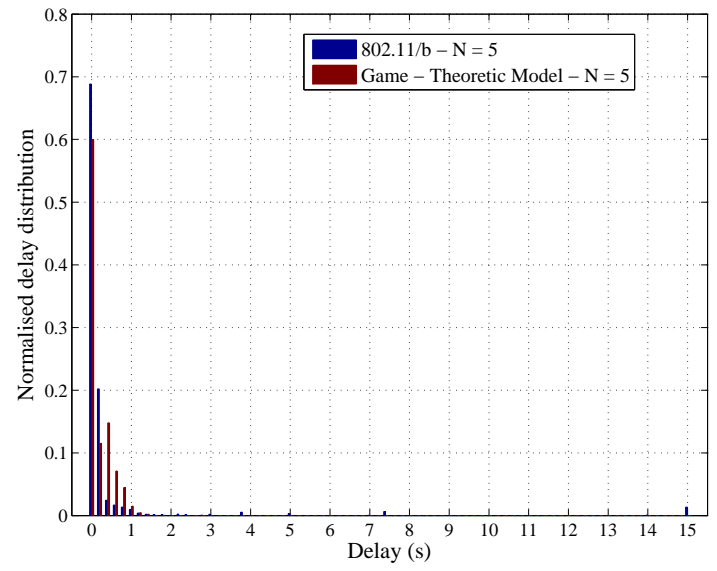

(a) Linear scale

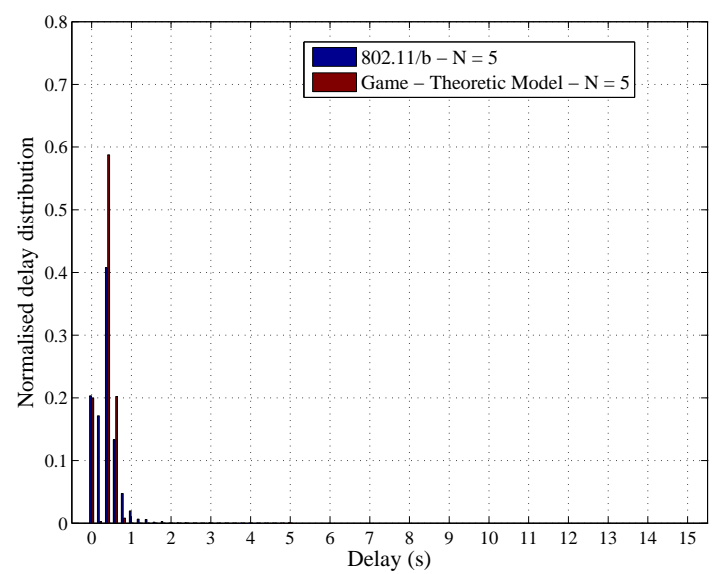

(c) Linear scale

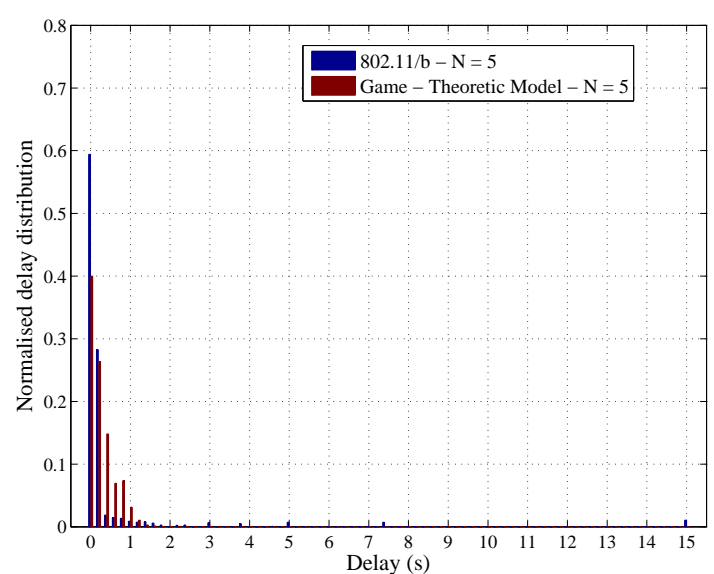

(e) Linear scale

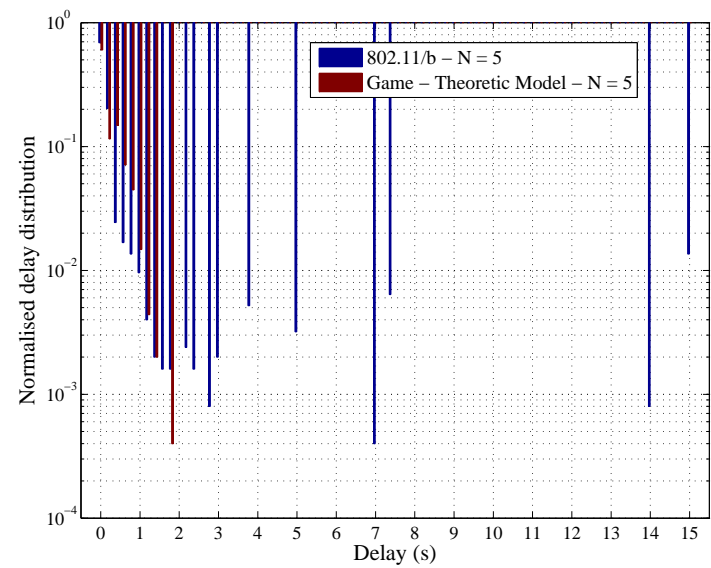

(b) Logarithmic scale

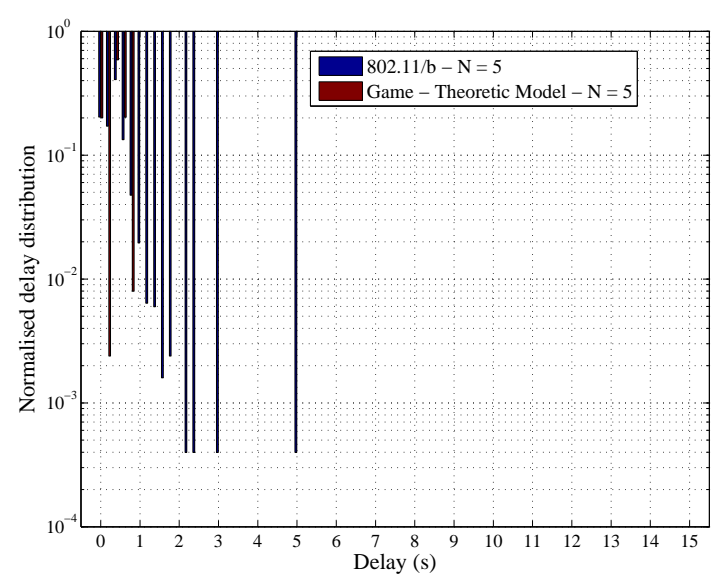

(d) Logarithmic scale

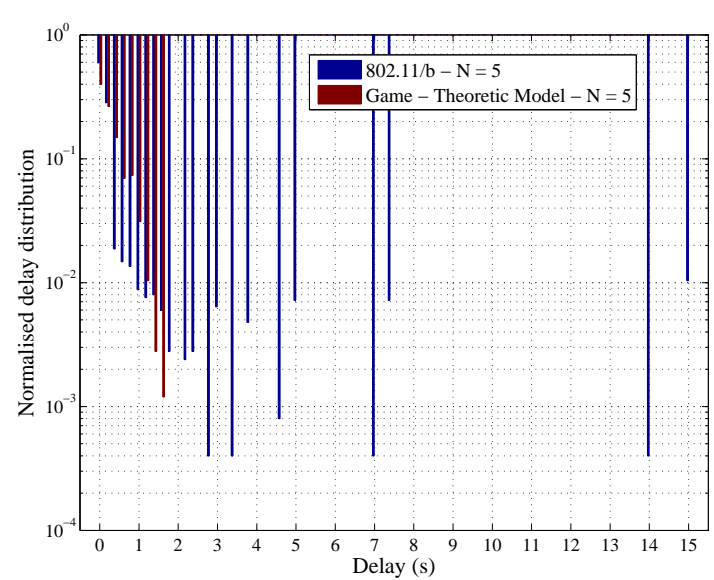

(f) Logarithmic scale

Figure B.2: Normalised delay distribution comparison for a network of 5 active nodes, operating in basic access method (DCF \& game-theoretic design) with 40-60 percent hidden terminal for each scenario. The bin-size is 0.2 seconds. 


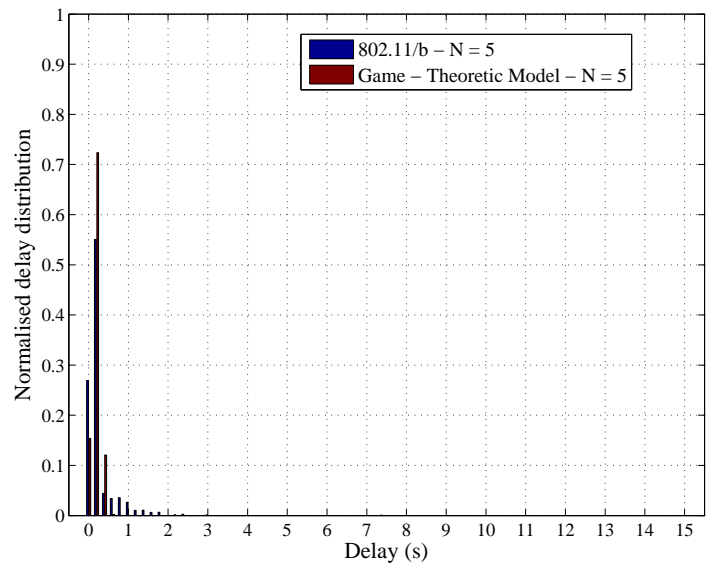

(a) Linear scale

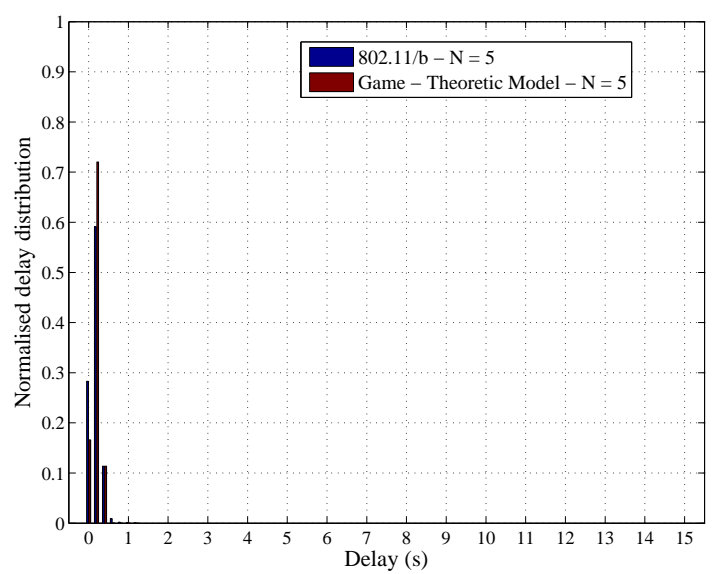

(c) Linear scale

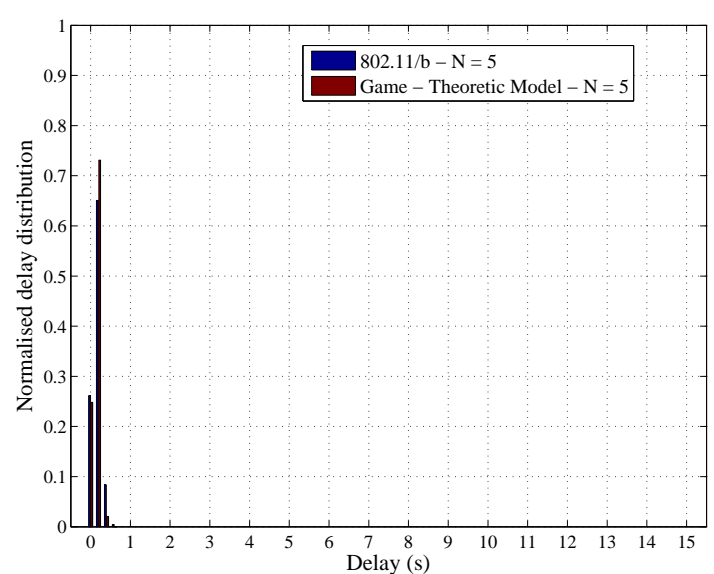

(e) Linear scale

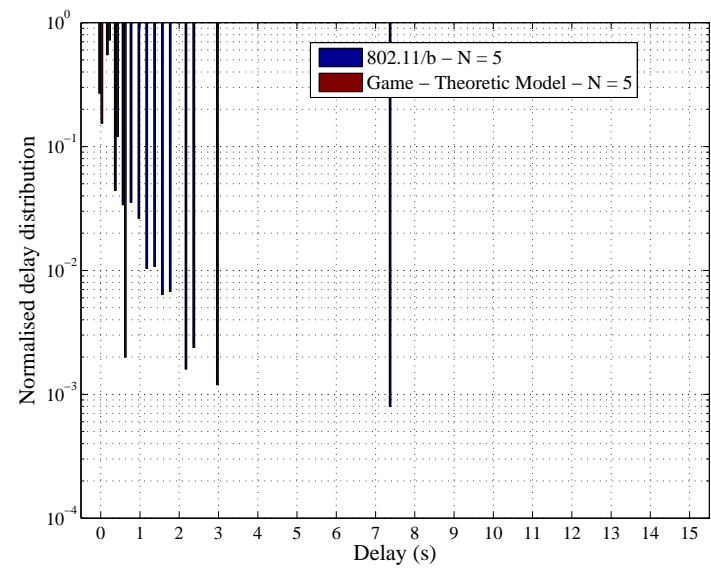

(b) Logarithmic scale

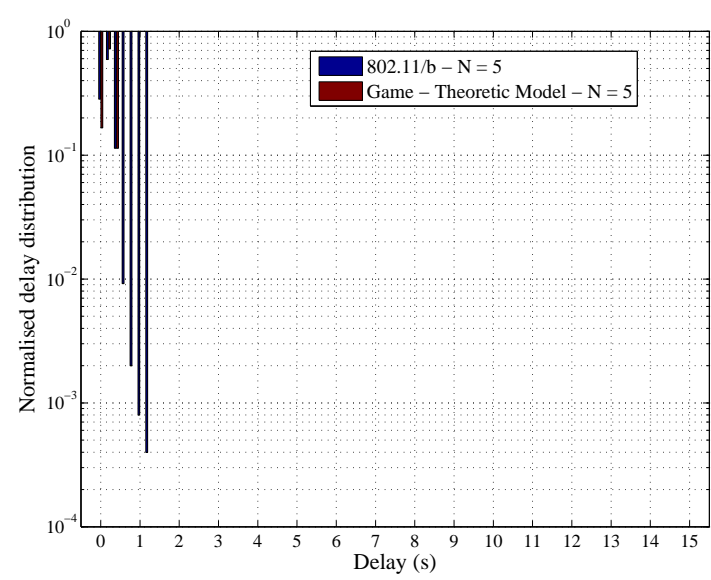

(d) Logarithmic scale

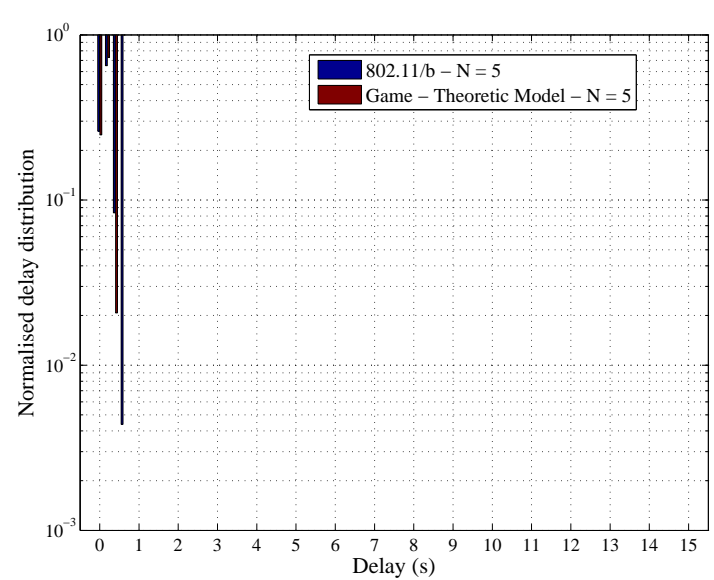

(f) Logarithmic scale

Figure B.3: Normalised delay distribution comparison for a network of 5 active nodes, operating in RTS/CTS access method (DCF \& game-theoretic design) with 10-30 percent hidden terminal for each scenario. The bin-size is 0.2 seconds. 


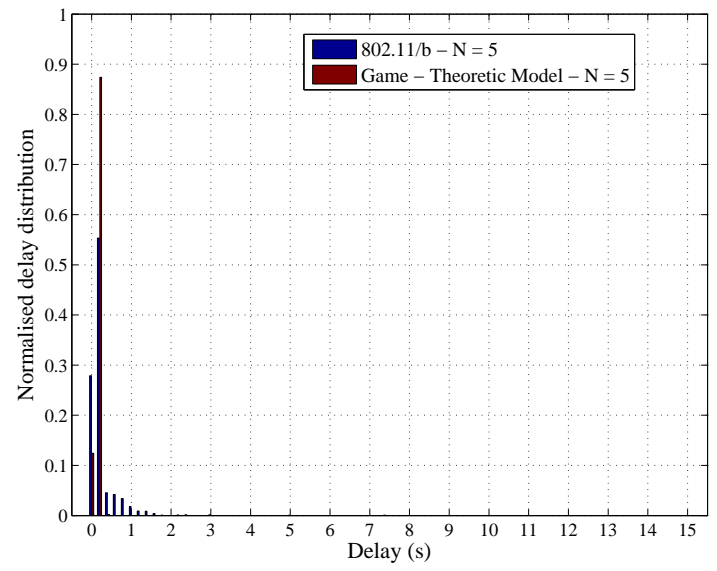

(a) Linear scale

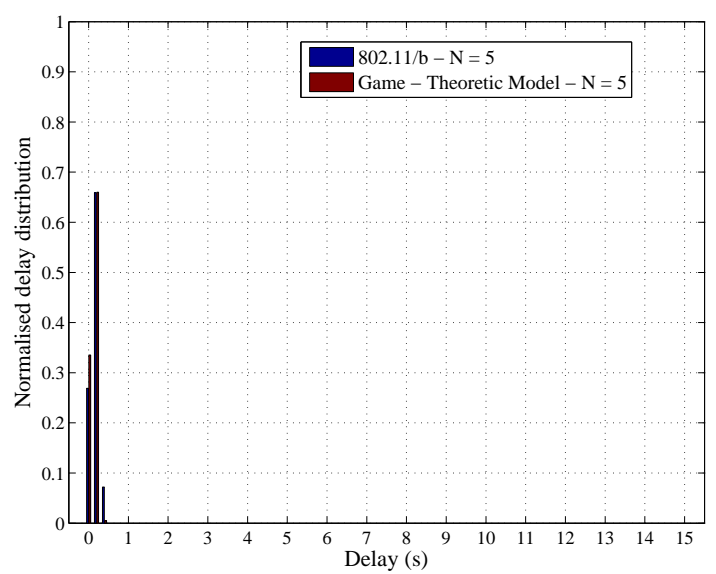

(c) Linear scale

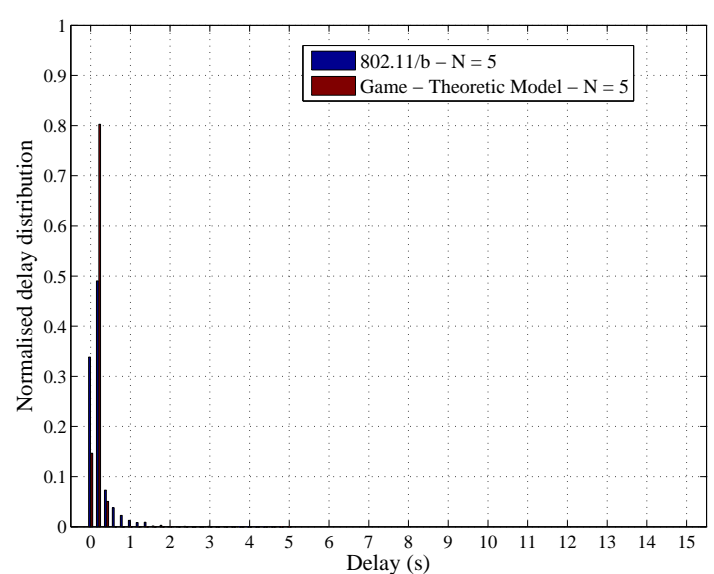

(e) Linear scale

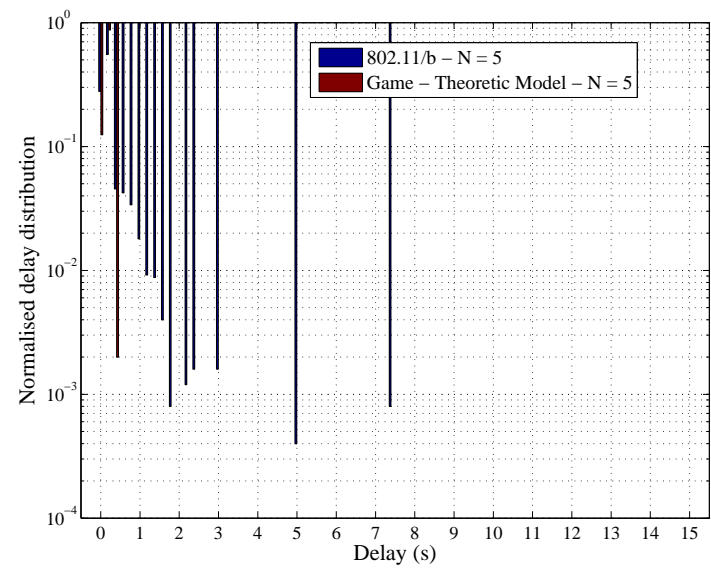

(b) Logarithmic scale

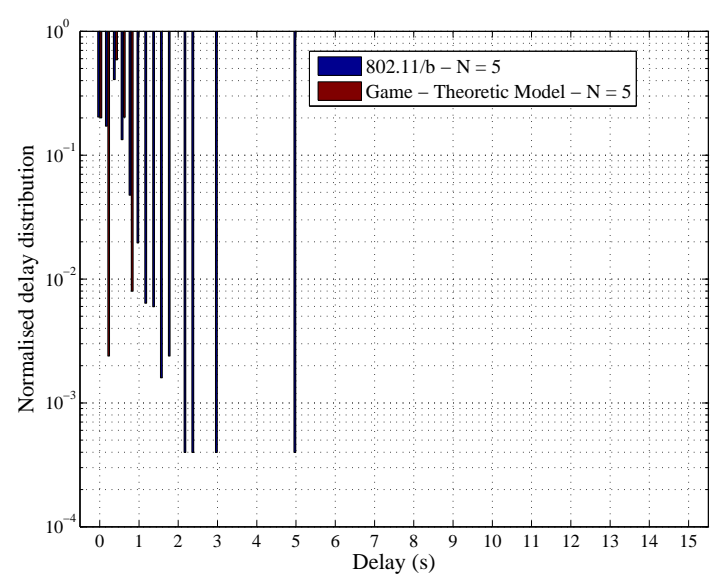

(d) Logarithmic scale

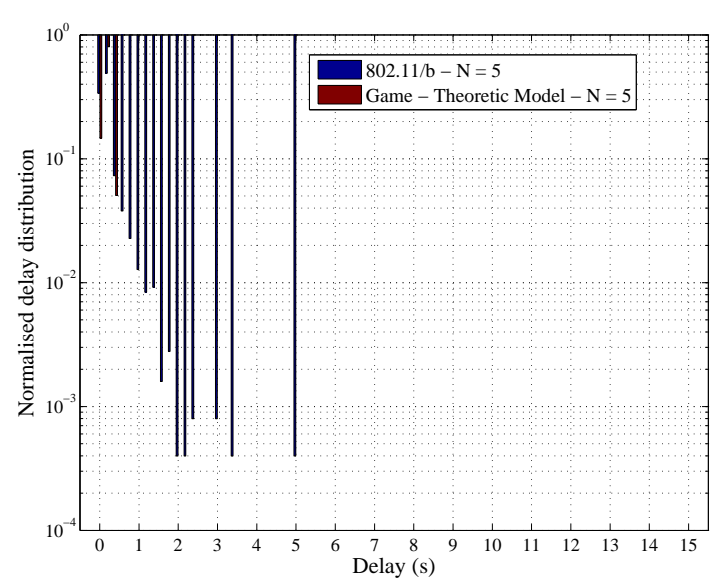

(f) Logarithmic scale

Figure B.4: Normalised delay distribution comparison for a network of 5 active nodes, operating in RTS/CTS access method (DCF \& game-theoretic design) with 40-60 percent hidden terminal for each scenario. The bin-size is 0.2 seconds. 


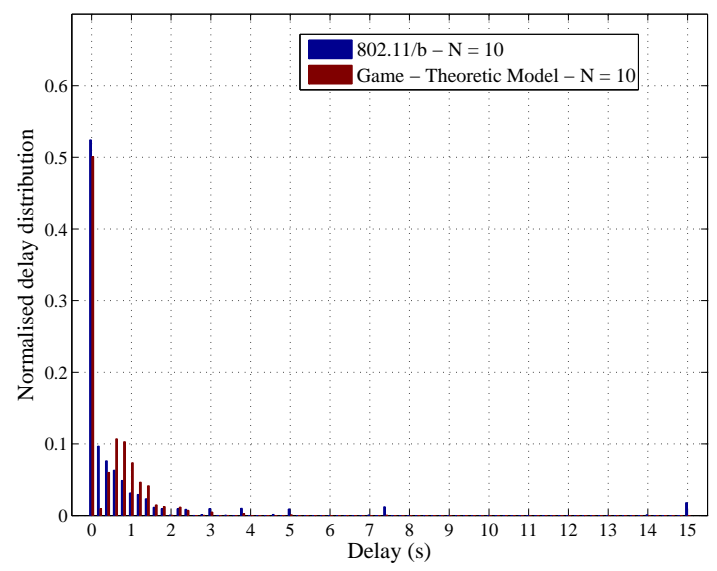

(a) Linear scale

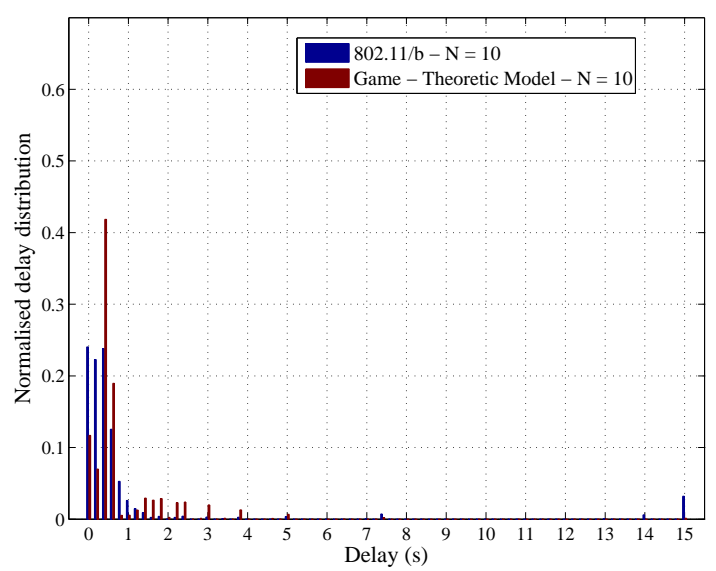

(c) Linear scale

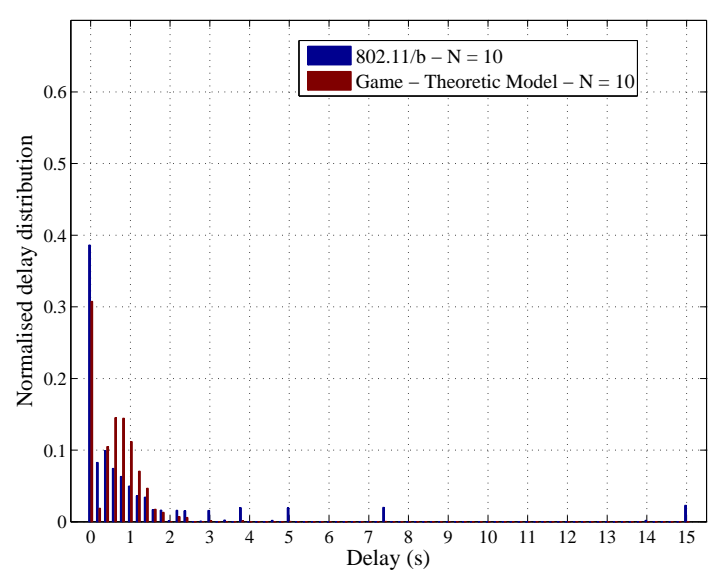

(e) Linear scale

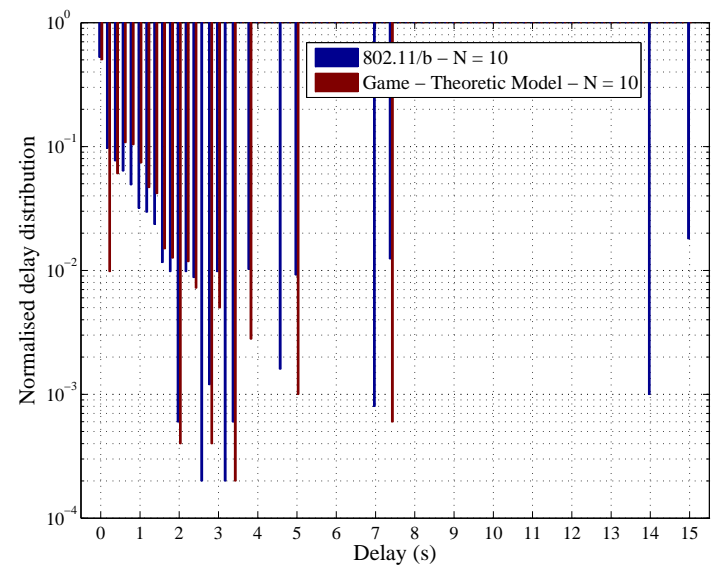

(b) Logarithmic scale

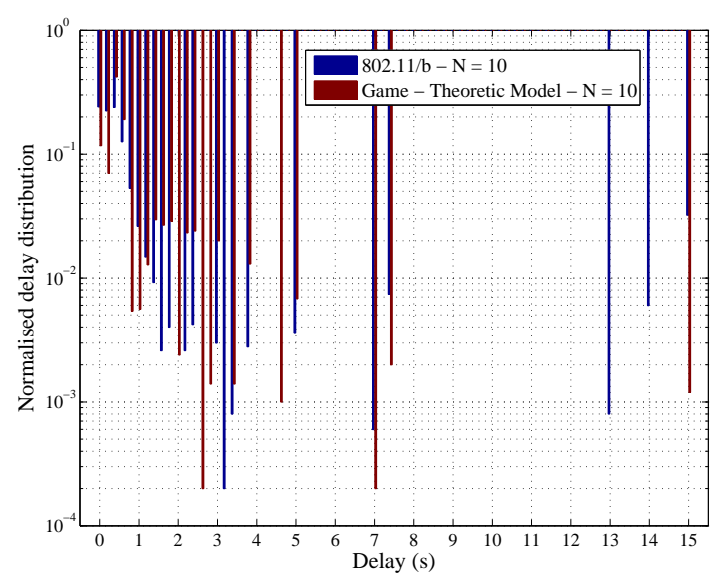

(d) Logarithmic scale

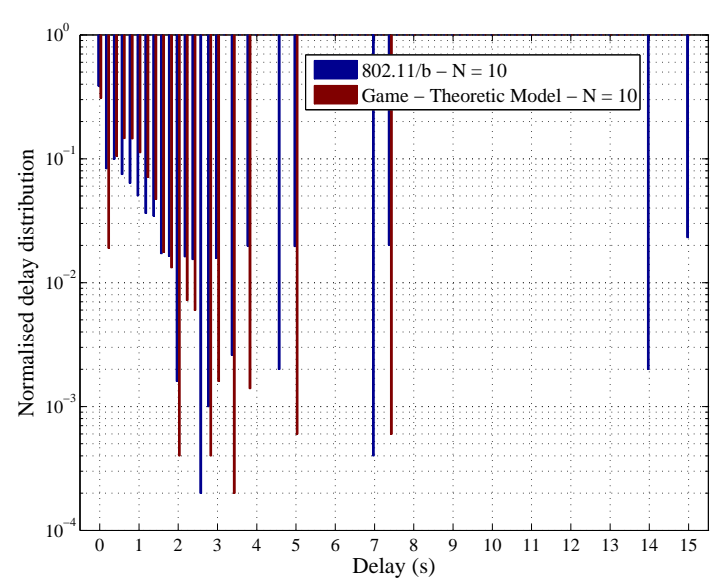

(f) Logarithmic scale

Figure B.5: Normalised delay distribution comparison for a network of 10 active nodes, operating in basic access method (DCF \& game-theoretic design) with 10-30 percent hidden terminal for each scenario. The bin-size is 0.2 seconds. 


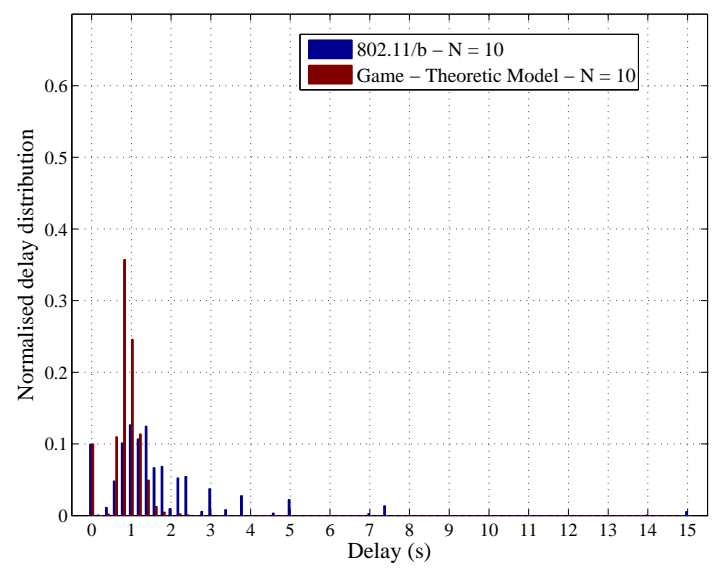

(a) Linear scale

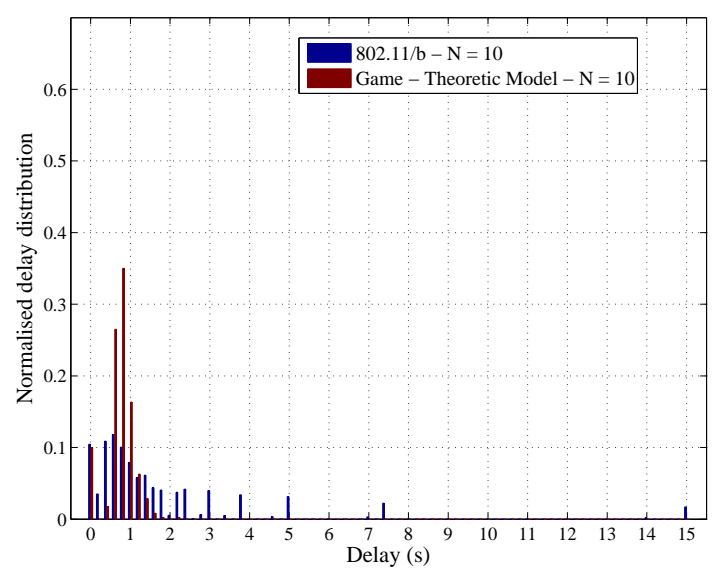

(c) Linear scale

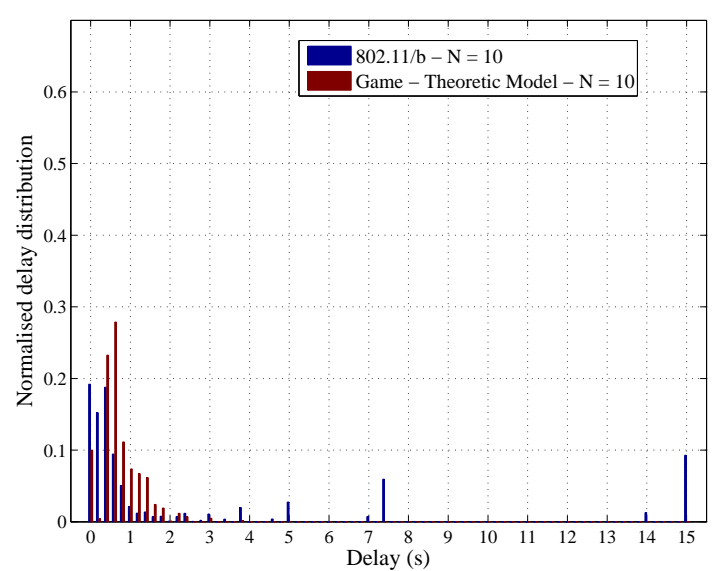

(e) Linear scale

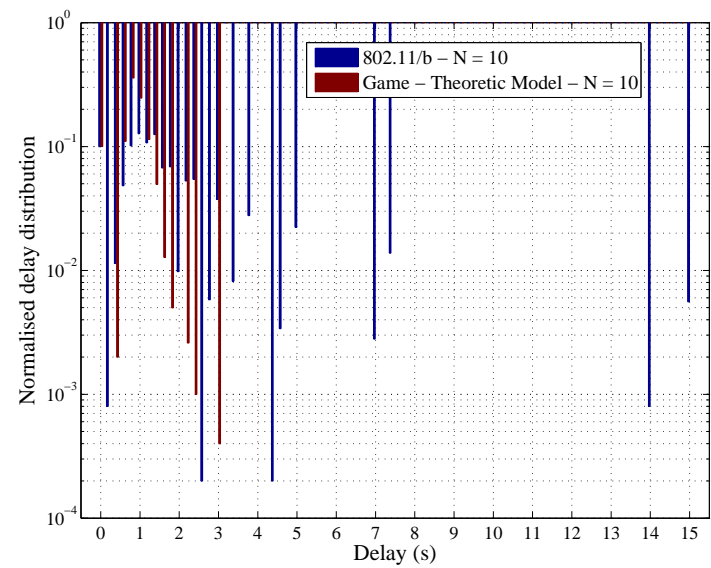

(b) Logarithmic scale

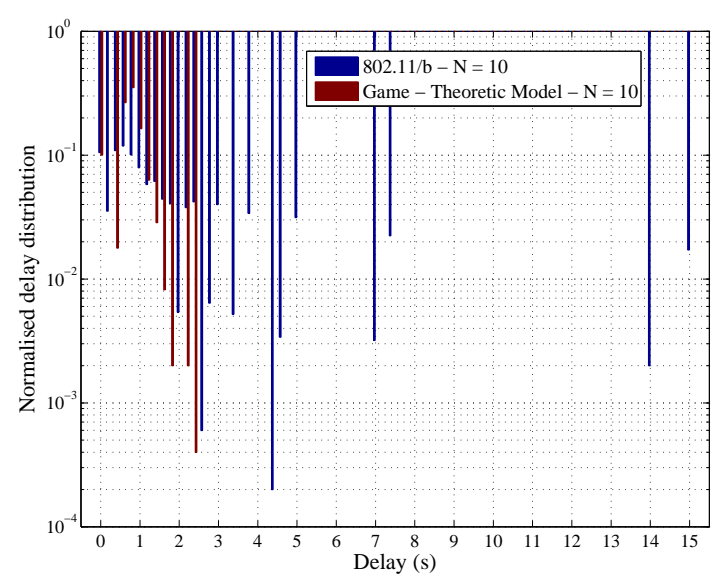

(d) Logarithmic scale

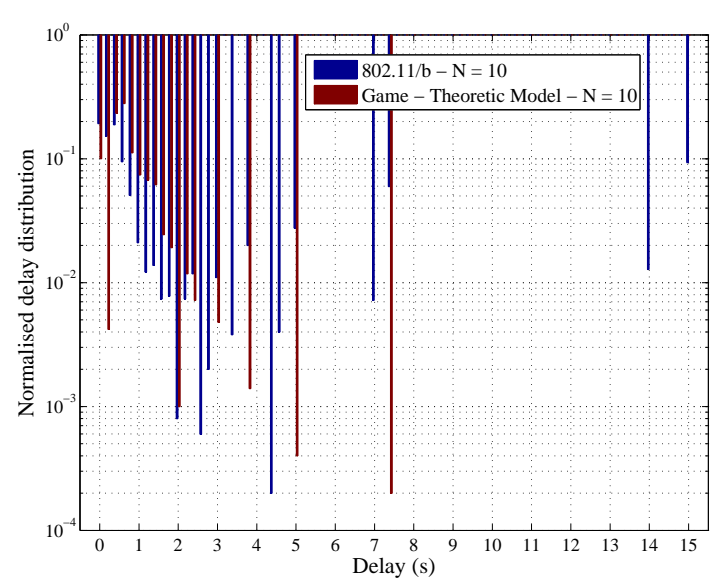

(f) Logarithmic scale

Figure B.6: Normalised delay distribution comparison for a network of 10 active nodes, operating in basic access method (DCF \& game-theoretic design) with 40-60 percent hidden terminal for each scenario. The bin-size is 0.2 seconds. 


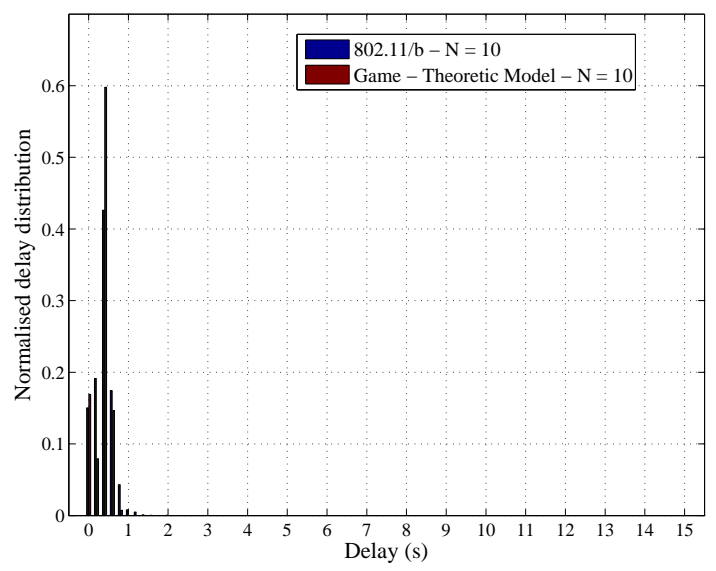

(a) Linear scale

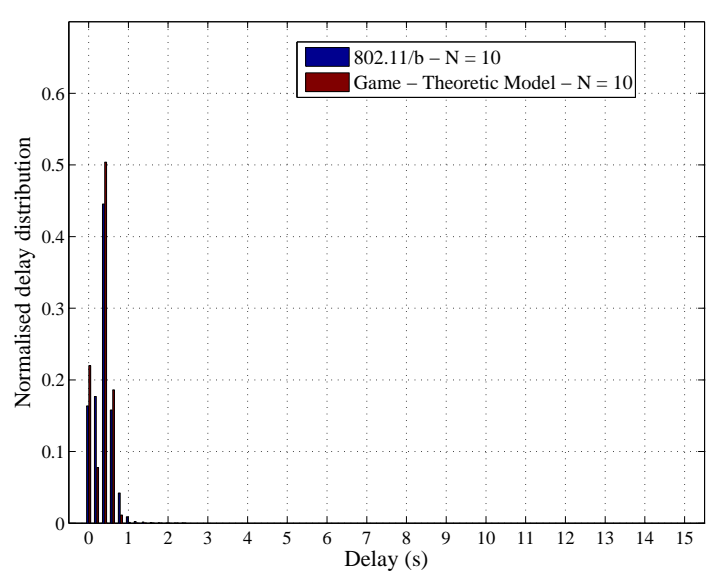

(c) Linear scale

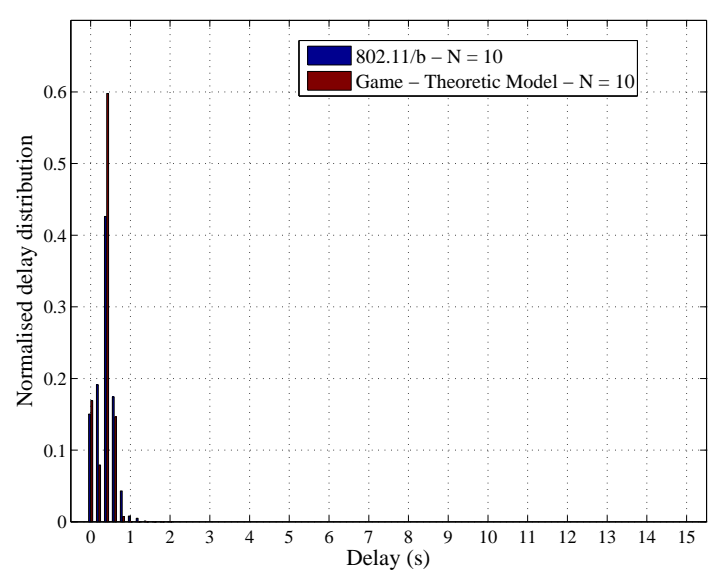

(e) Linear scale

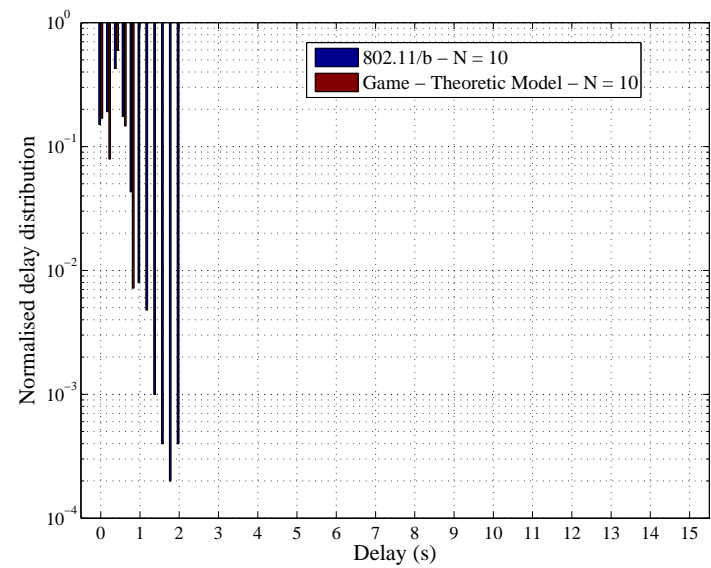

(b) Logarithmic scale

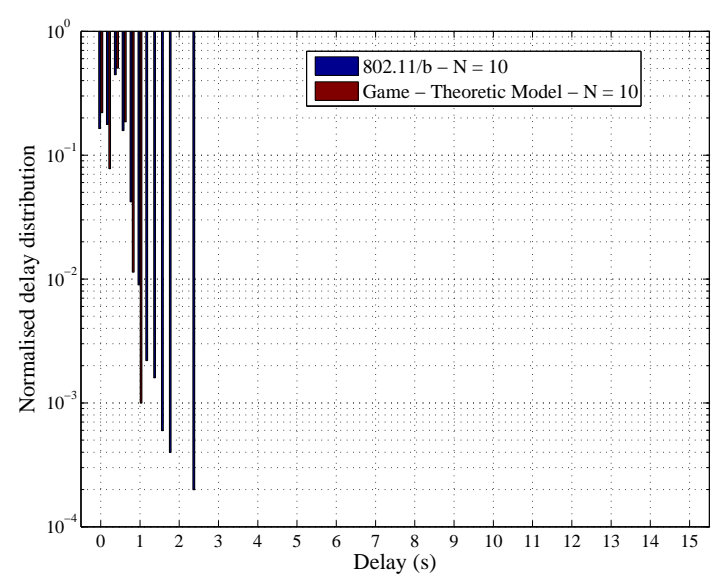

(d) Logarithmic scale

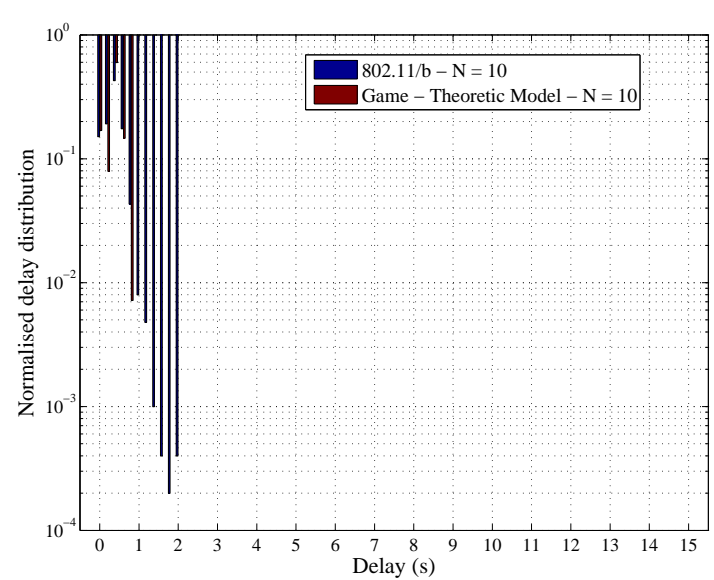

(f) Logarithmic scale

Figure B.7: Normalised delay distribution comparison for a network of 10 active nodes, operating in RTS/CTS access method (DCF \& game-theoretic design) with 10-30 percent hidden terminal for each scenario. The bin-size is 0.2 seconds. 


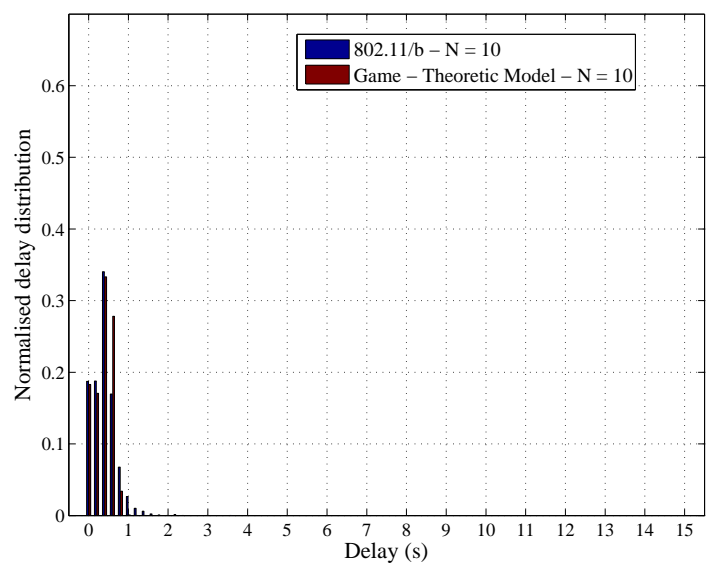

(a) Linear scale

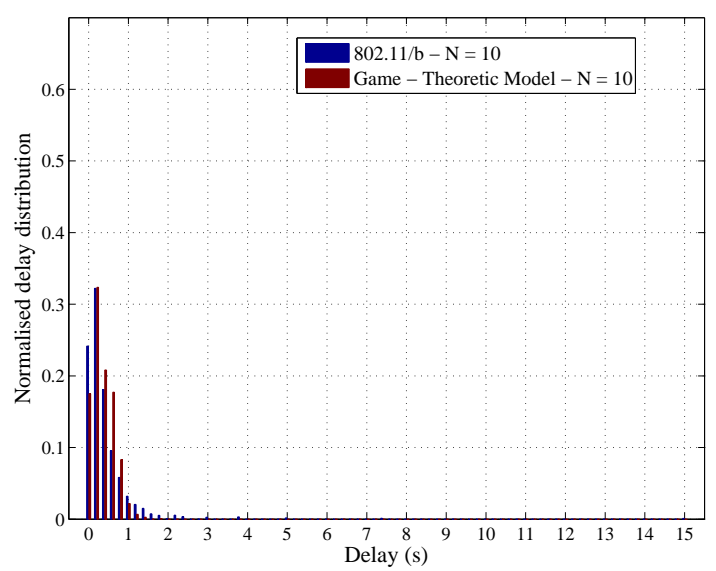

(c) Linear scale

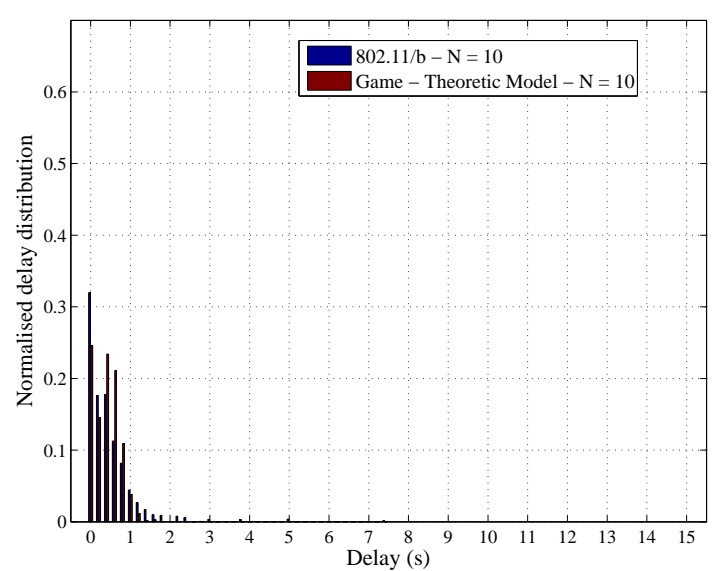

(e) Linear scale

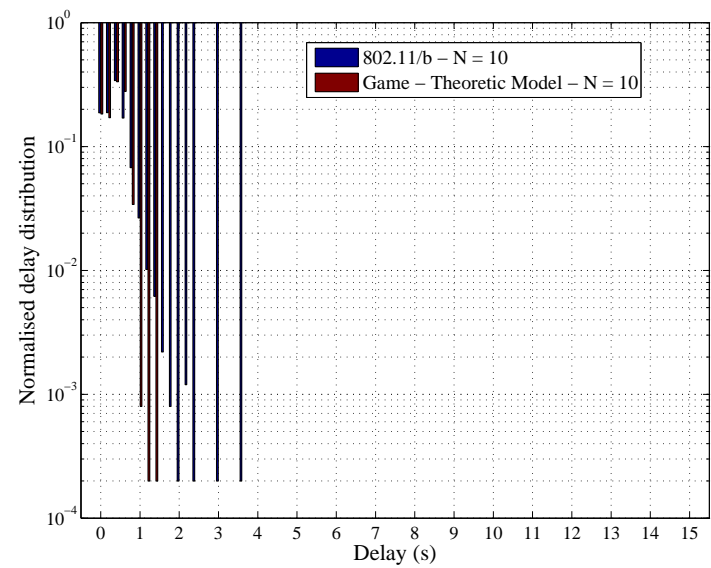

(b) Logarithmic scale

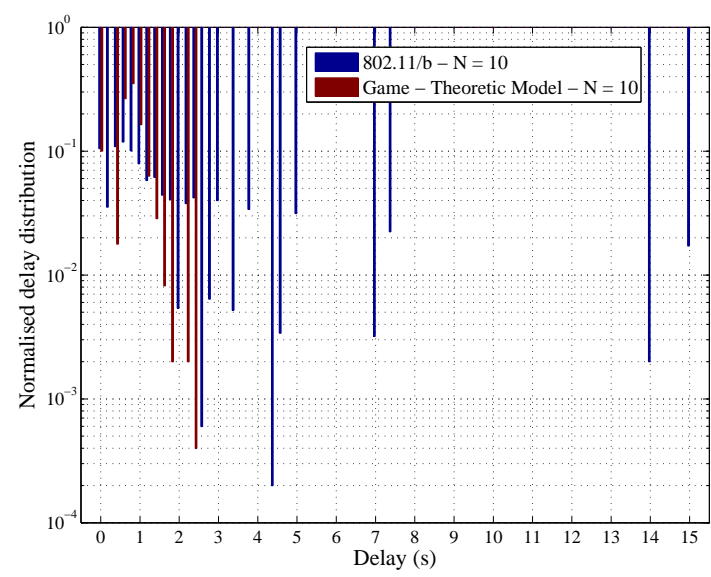

(d) Logarithmic scale

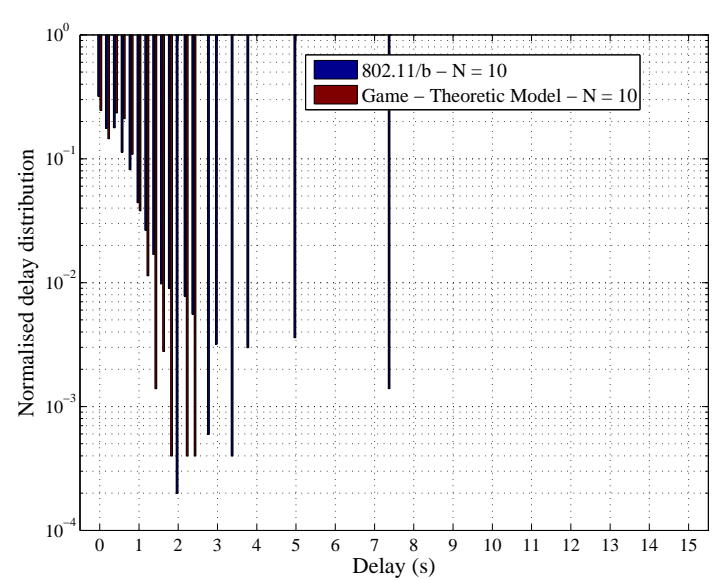

(f) Logarithmic scale

Figure B.8: Normalised delay distribution comparison for a network of 10 active nodes, operating in RTS/CTS access method (DCF \& game-theoretic design) with 40-60 percent hidden terminal for each scenario. The bin-size is 0.2 seconds. 


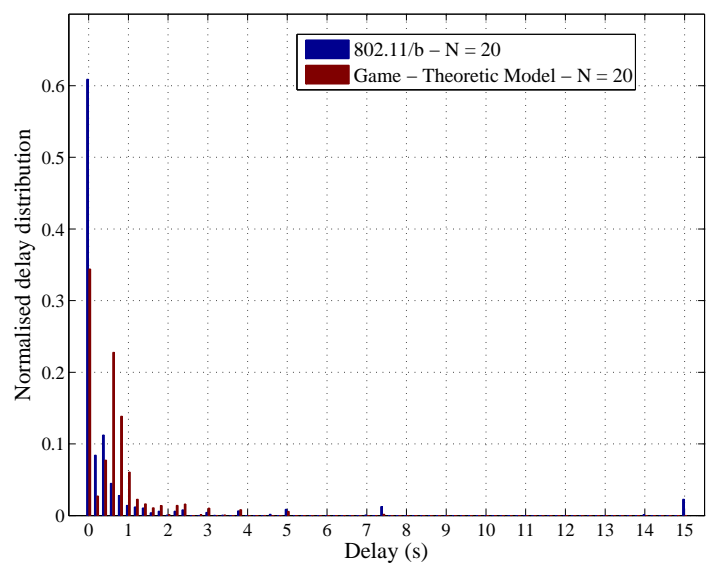

(a) Linear scale

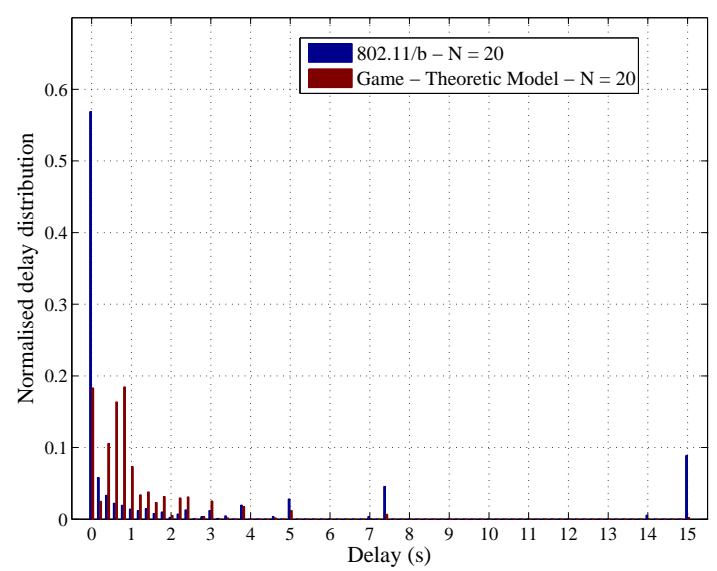

(c) Linear scale

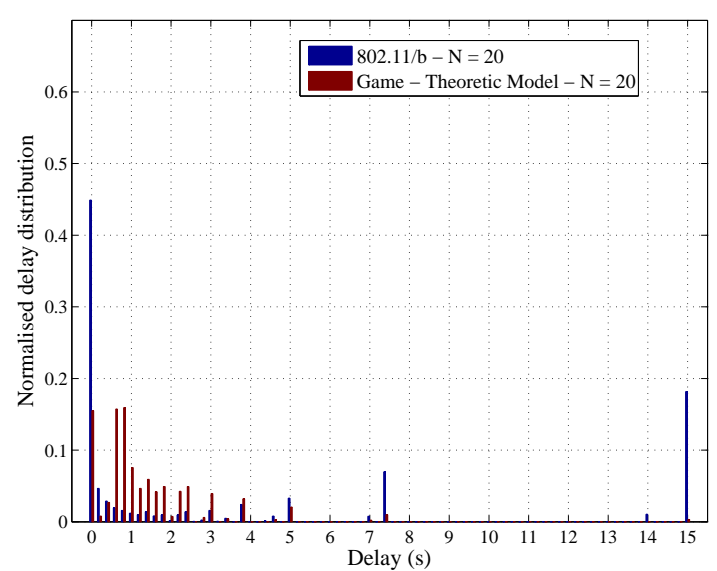

(e) Linear scale

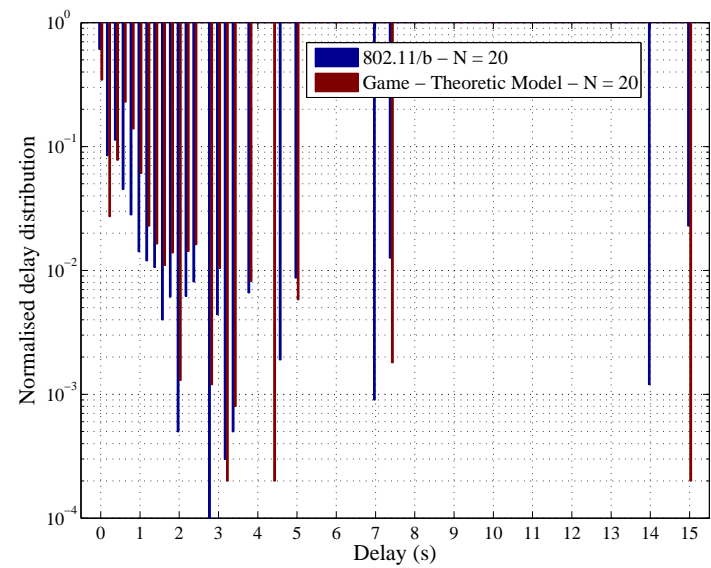

(b) Logarithmic scale

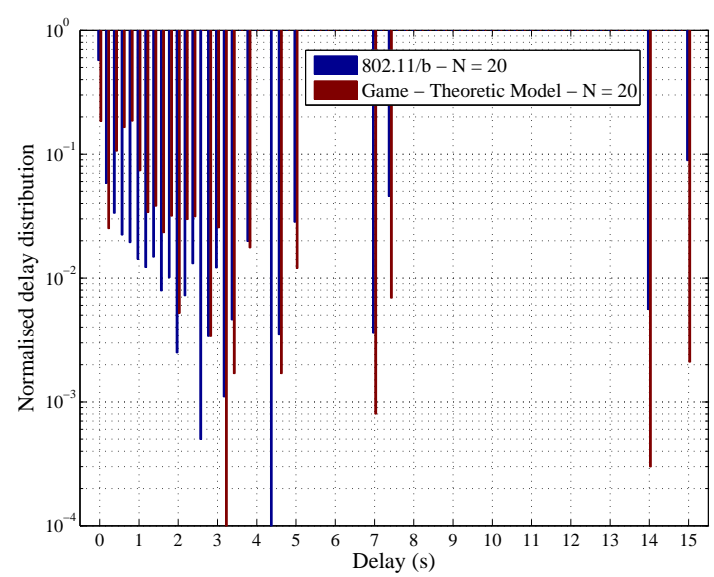

(d) Logarithmic scale

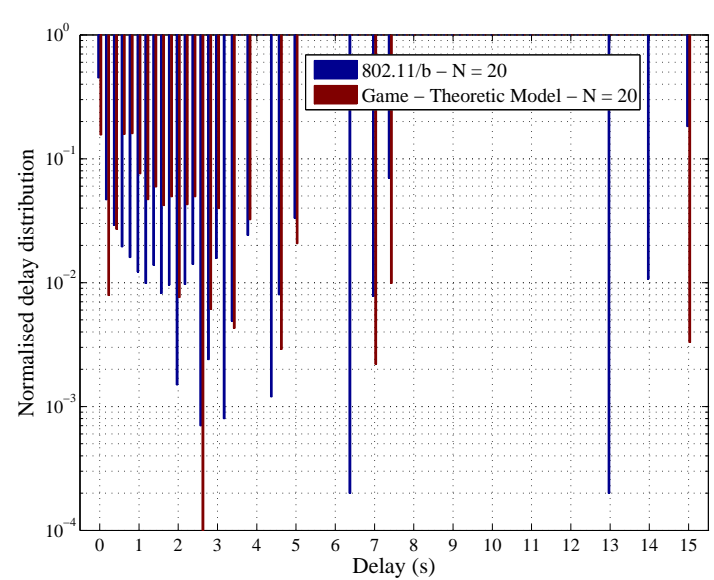

(f) Logarithmic scale

Figure B.9: Normalised delay distribution comparison for a network of 20 active nodes, operating in basic access method (DCF \& game-theoretic design) with 10-30 percent hidden terminal for each scenario. The bin-size is 0.2 seconds. 


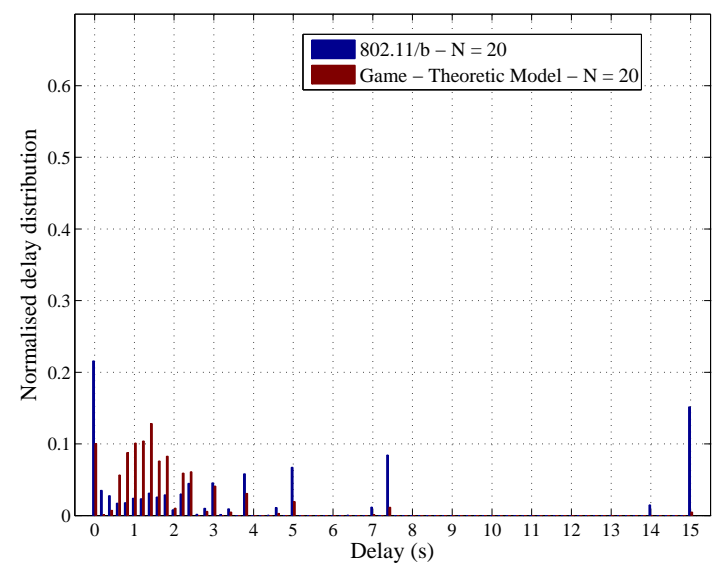

(a) Linear scale

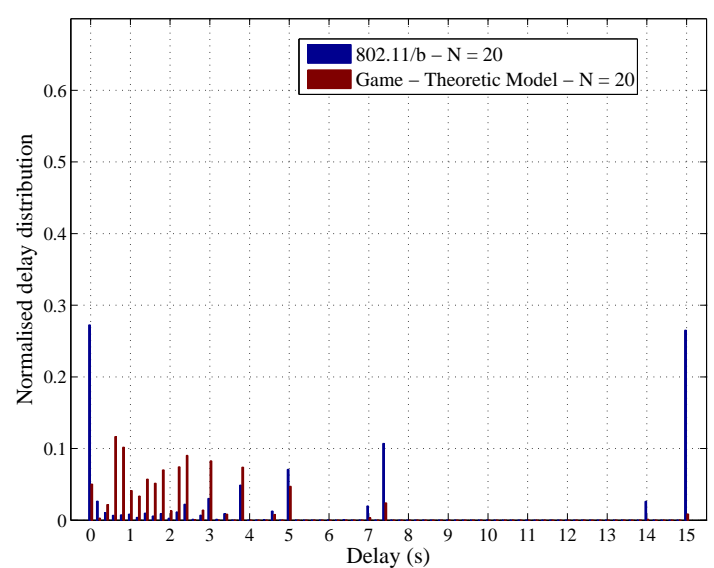

(c) Linear scale

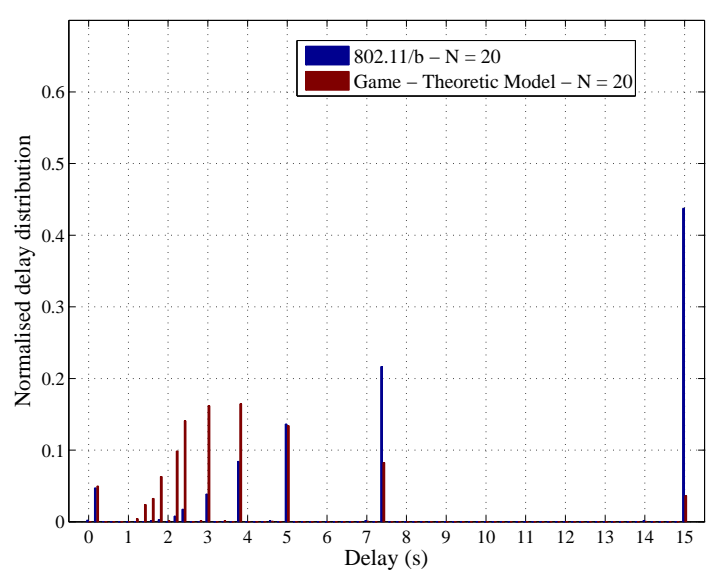

(e) Linear scale

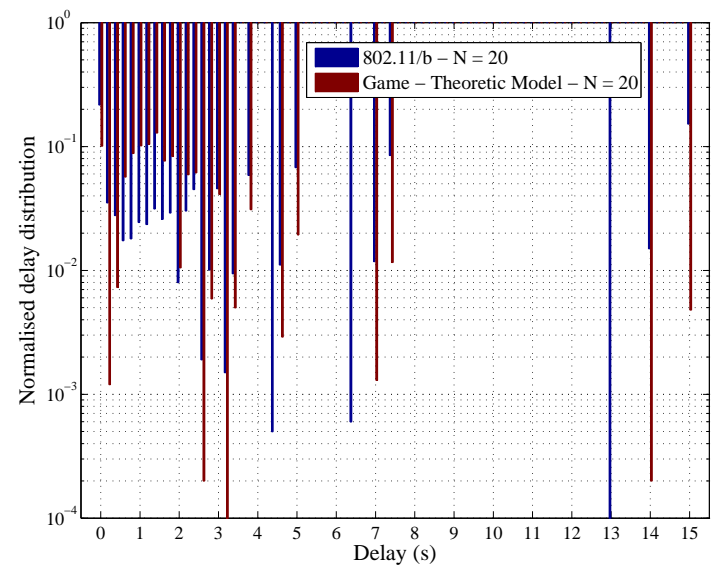

(b) Logarithmic scale

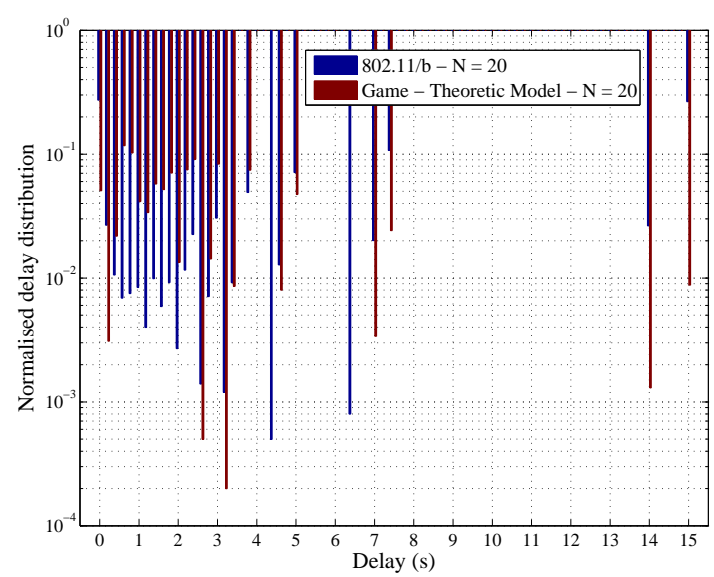

(d) Logarithmic scale

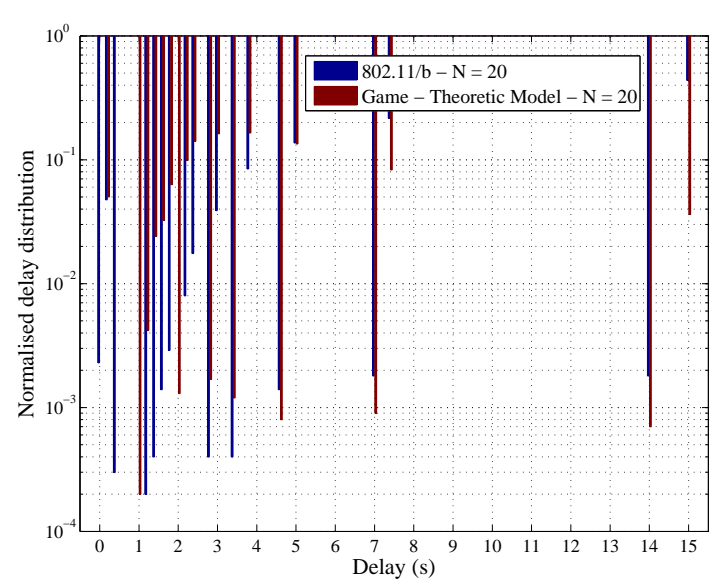

(f) Logarithmic scale

Figure B.10: Normalised delay distribution comparison for a network of 20 active nodes, operating in basic access method (DCF \& game-theoretic design) with 40-60 percent hidden terminal for each scenario. The bin-size is 0.2 seconds. 


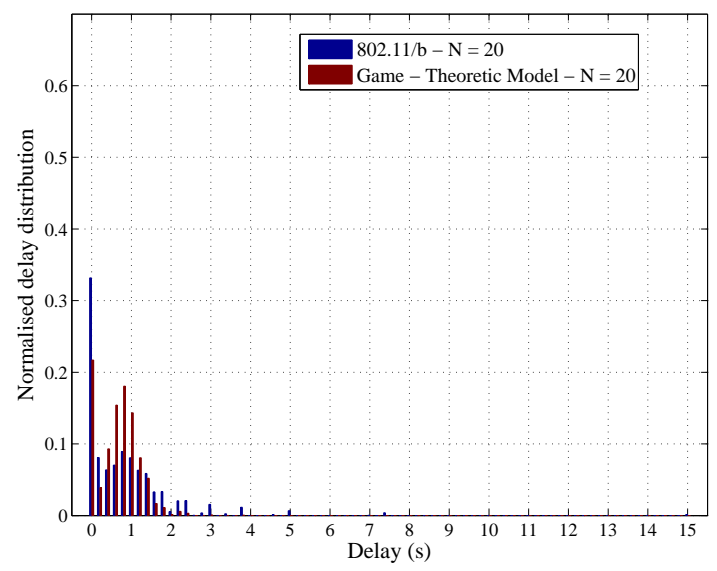

(a) Linear scale

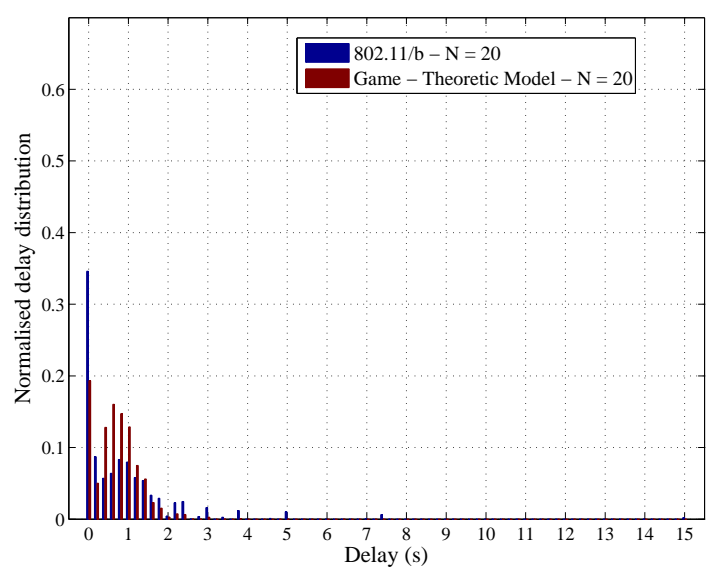

(c) Linear scale

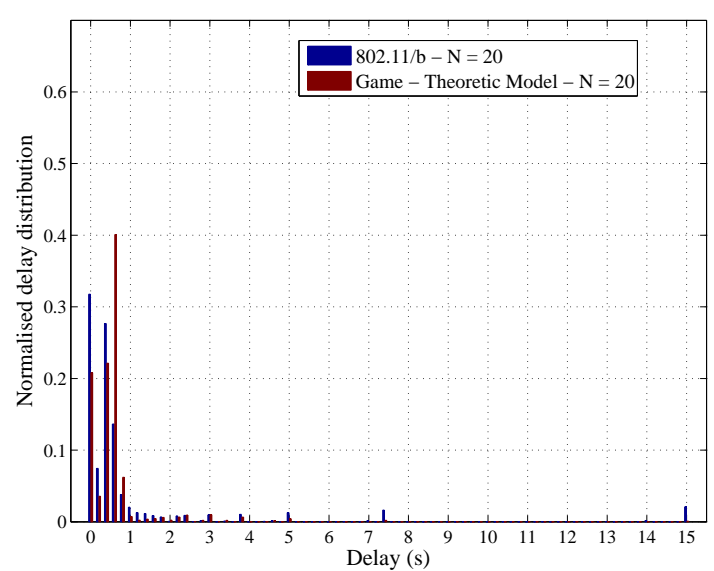

(e) Linear scale

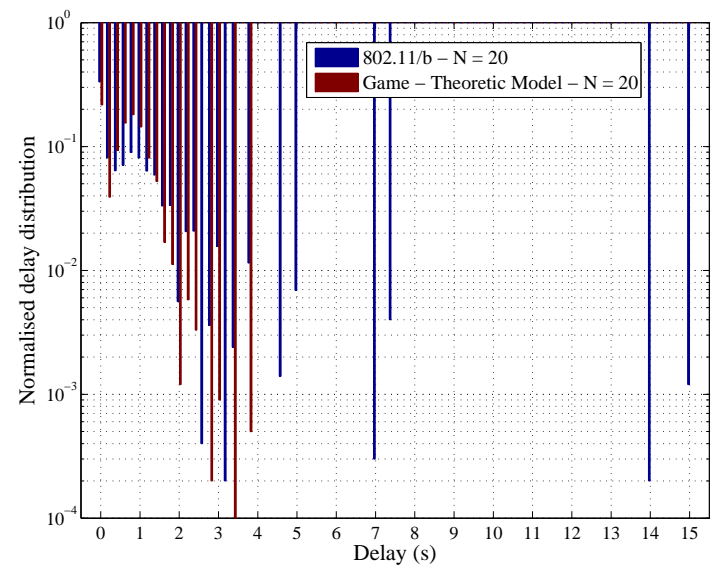

(b) Logarithmic scale

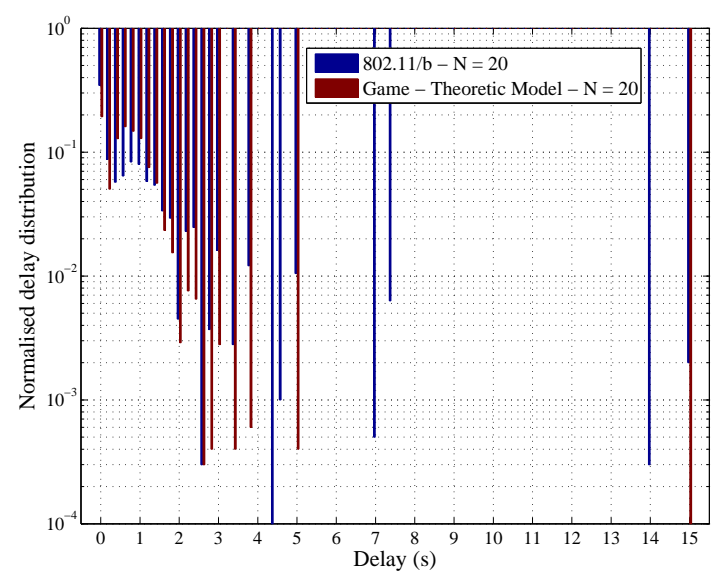

(d) Logarithmic scale

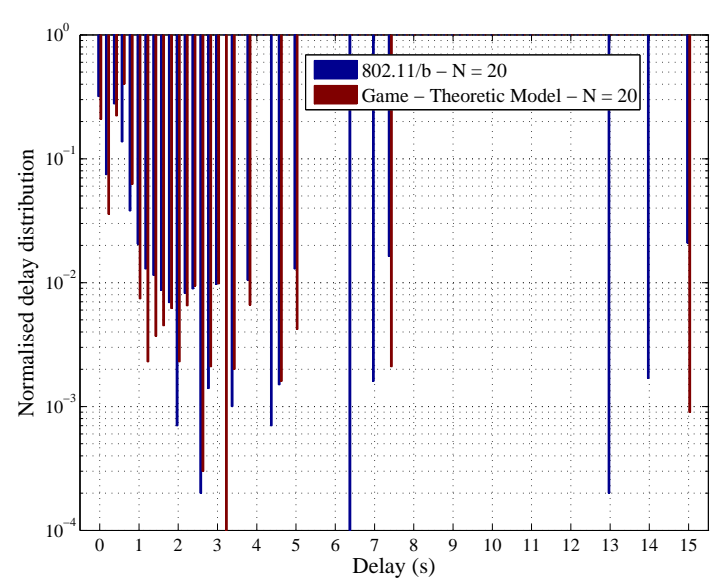

(f) Logarithmic scale

Figure B.11: Normalised delay distribution comparison for a network of 10 active nodes, operating in RTS/CTS access method (DCF \& game-theoretic design) with 10-30 percent hidden terminal for each scenario. The bin-size is 0.2 seconds. 


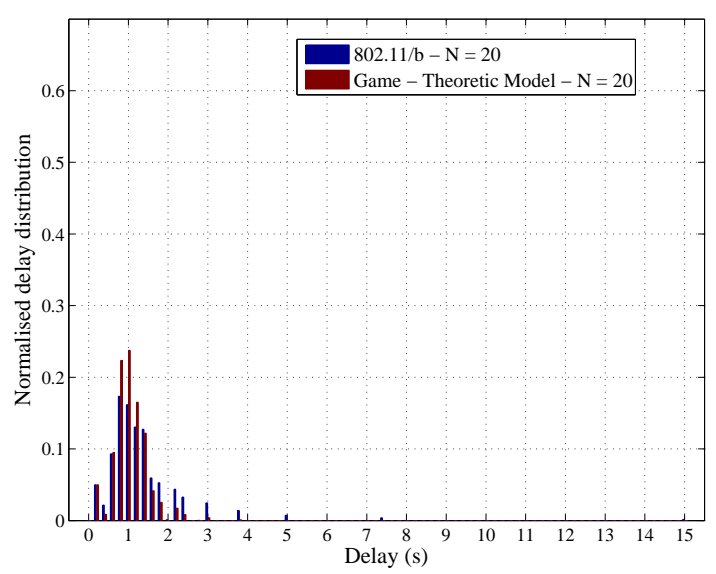

(a) Linear scale

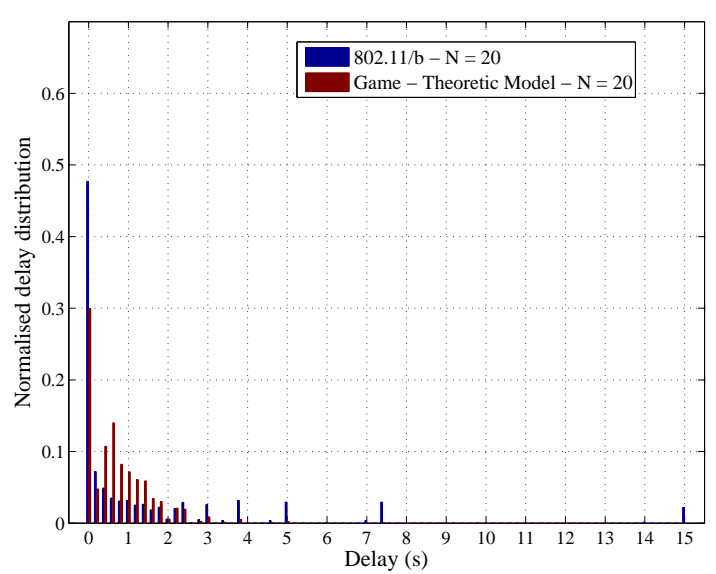

(c) Linear scale

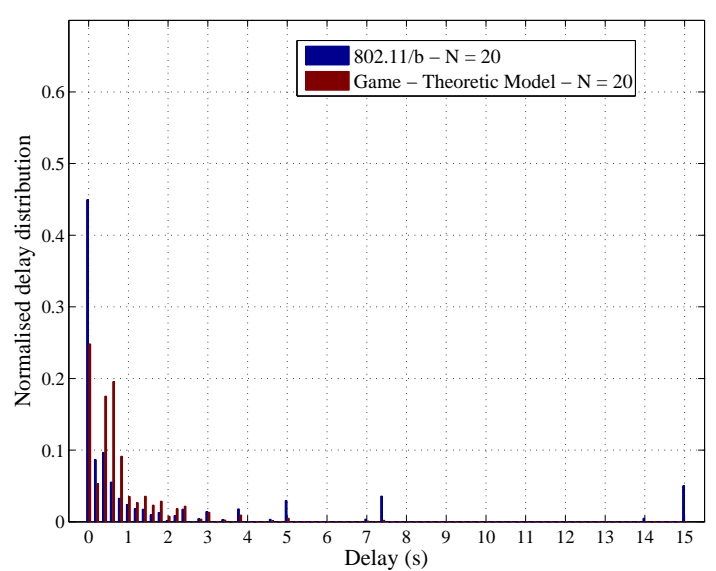

(e) Linear scale

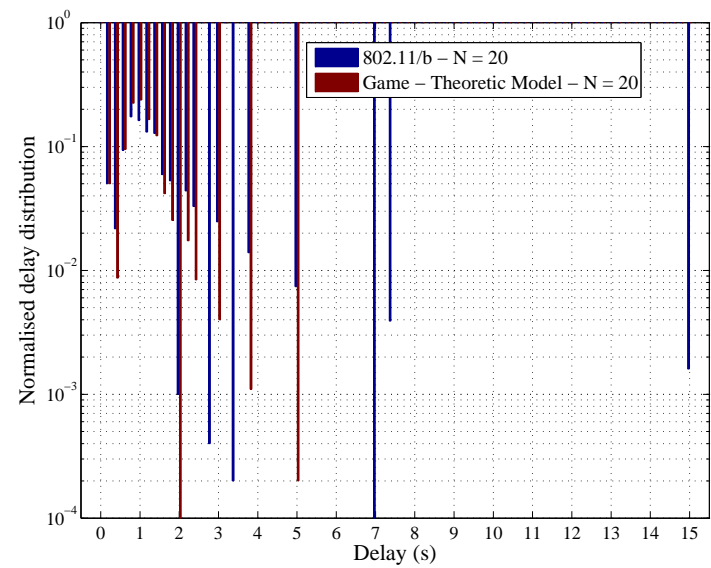

(b) Logarithmic scale

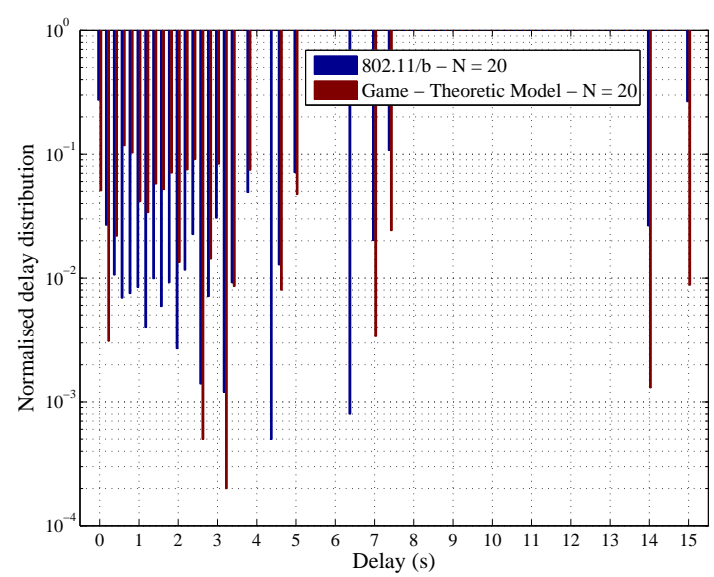

(d) Logarithmic scale

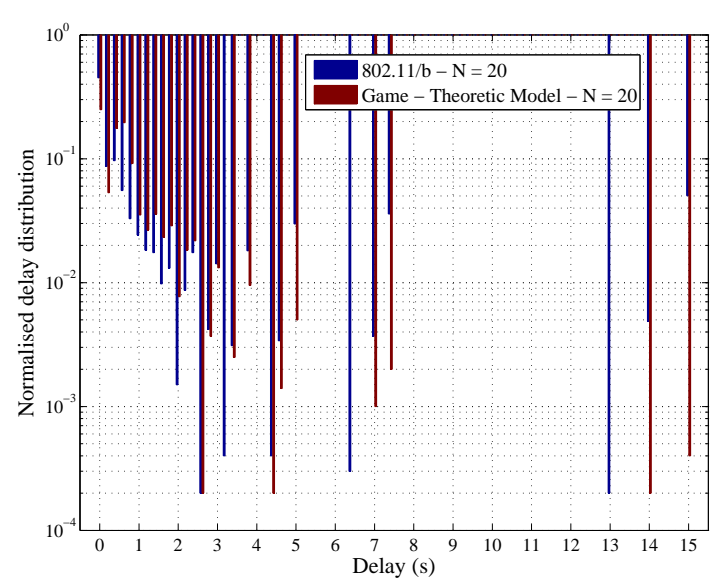

(f) Logarithmic scale

Figure B.12: Normalised delay distribution comparison for a network of 20 active nodes, operating in RTS/CTS access method (DCF \& game-theoretic design) with 40-60 percent hidden terminal for each scenario. The bin-size is 0.2 seconds. 


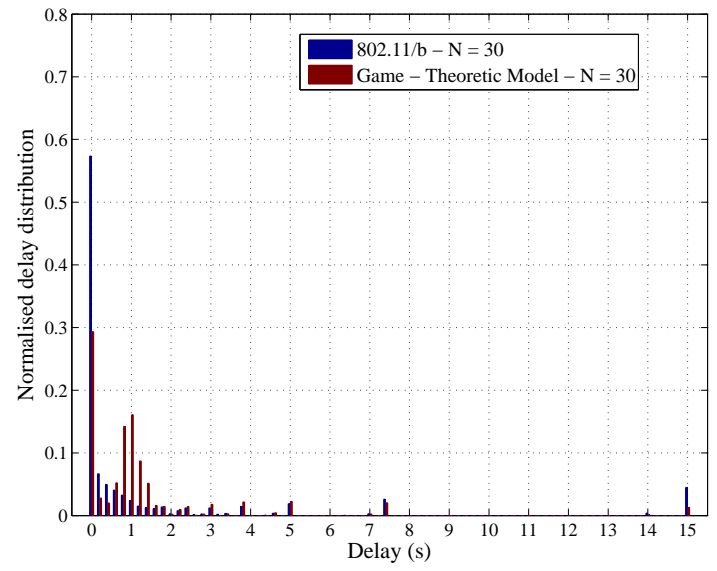

(a) Linear scale

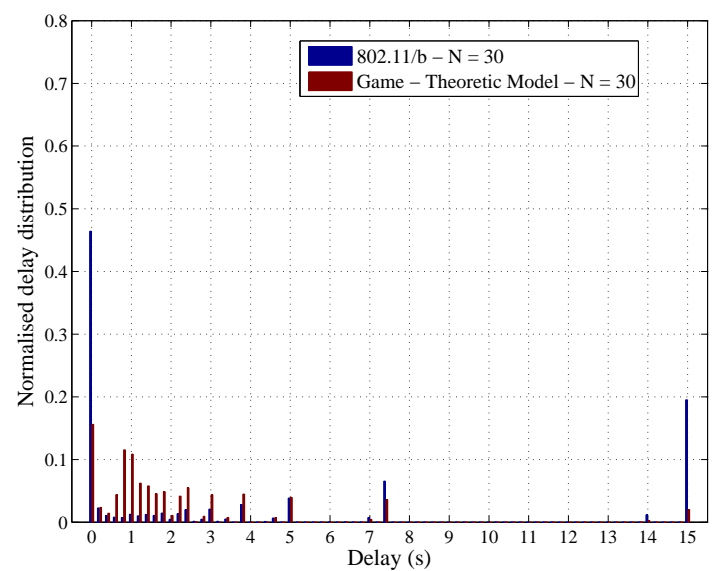

(c) Linear scale

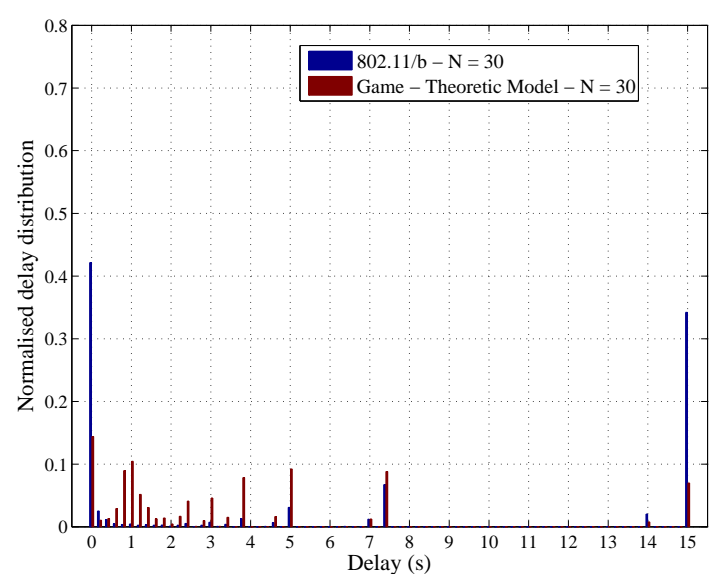

(e) Linear scale

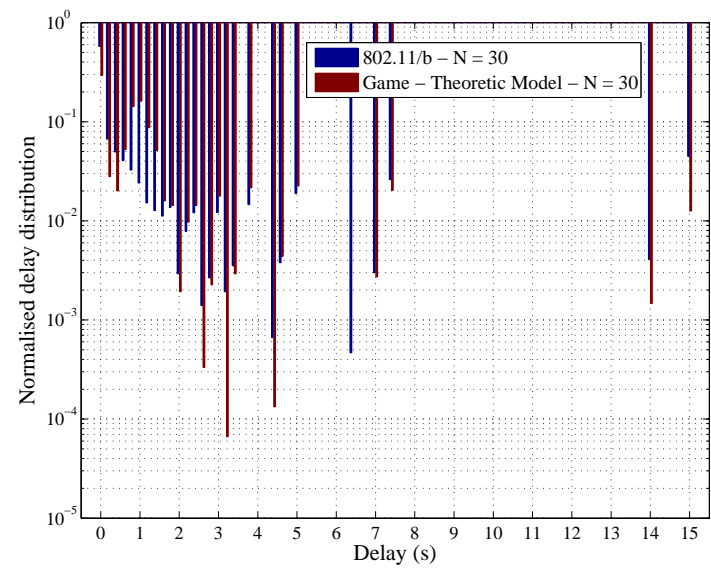

(b) Logarithmic scale

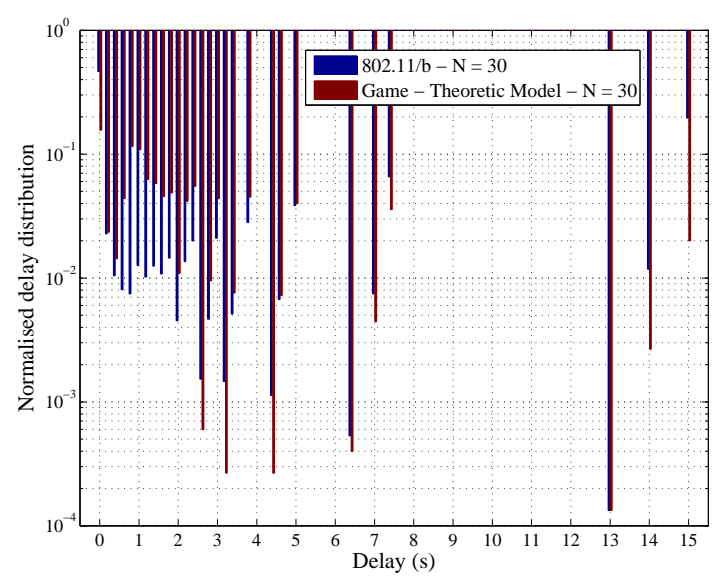

(d) Logarithmic scale

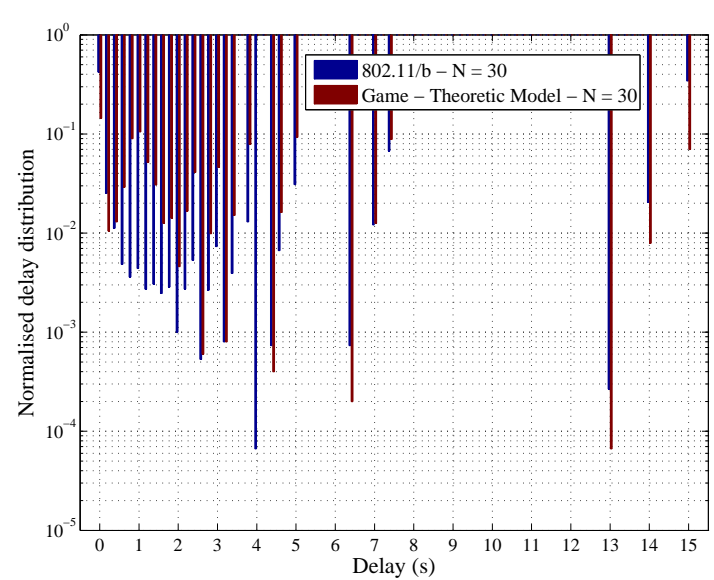

(f) Logarithmic scale

Figure B.13: Normalised delay distribution comparison for a network of 30 active nodes, operating in basic access method (DCF \& game-theoretic design) with 10-30 percent hidden terminal for each scenario. The bin-size is 0.2 seconds. 


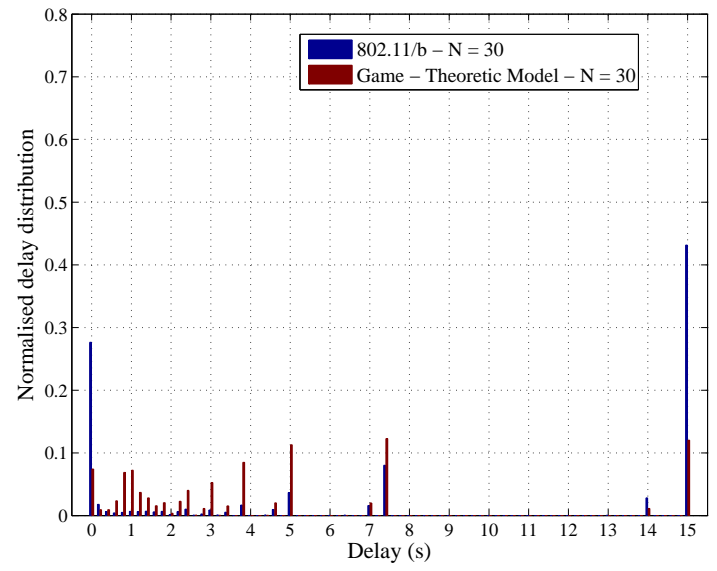

(a) Linear scale

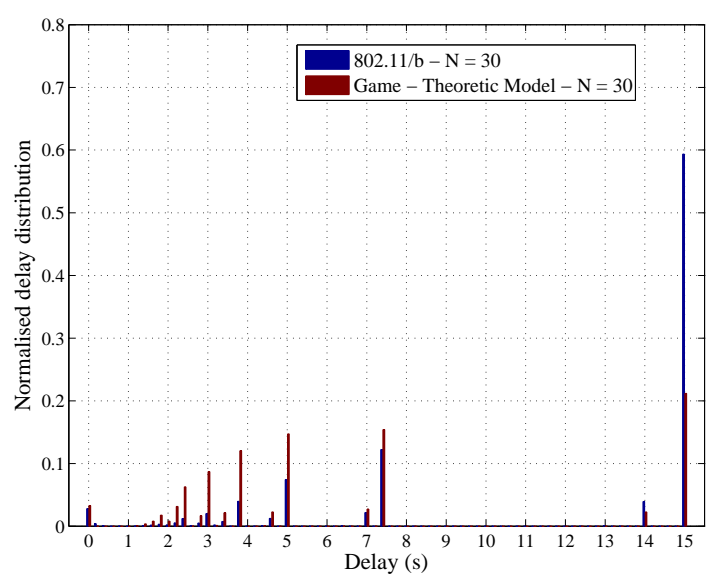

(c) Linear scale

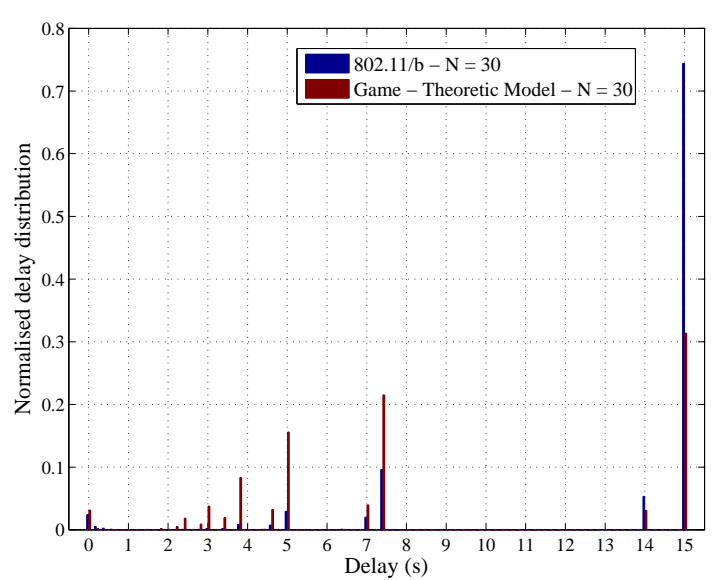

(e) Linear scale

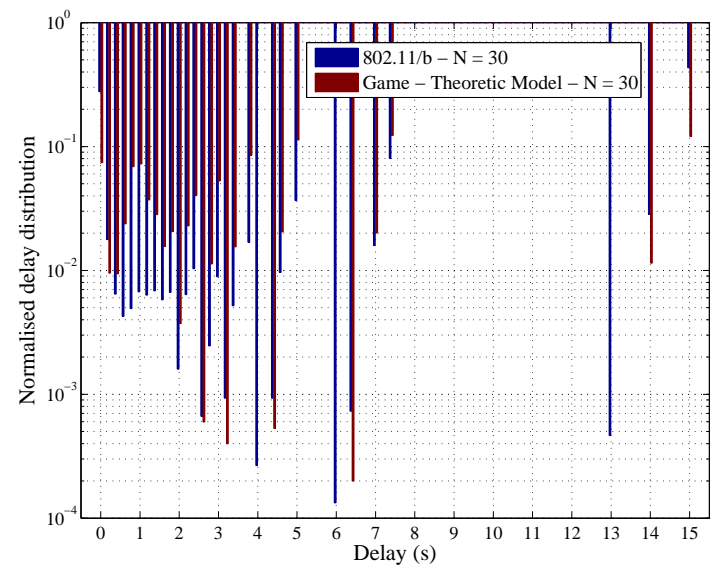

(b) Logarithmic scale

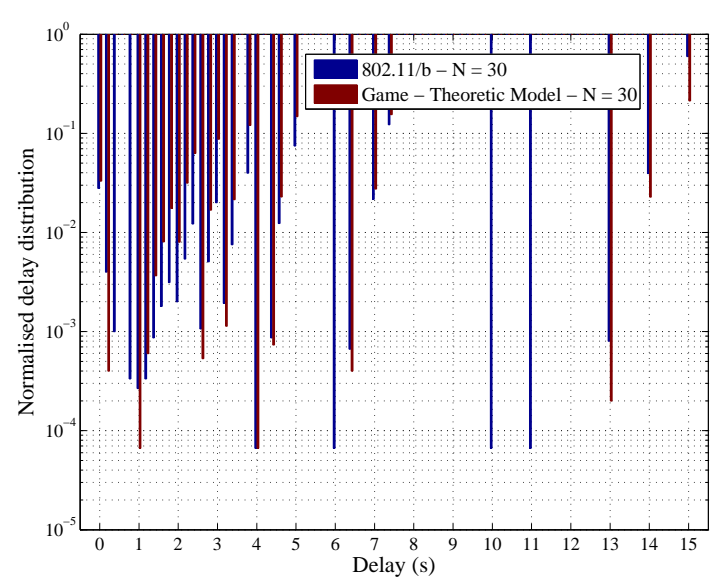

(d) Logarithmic scale

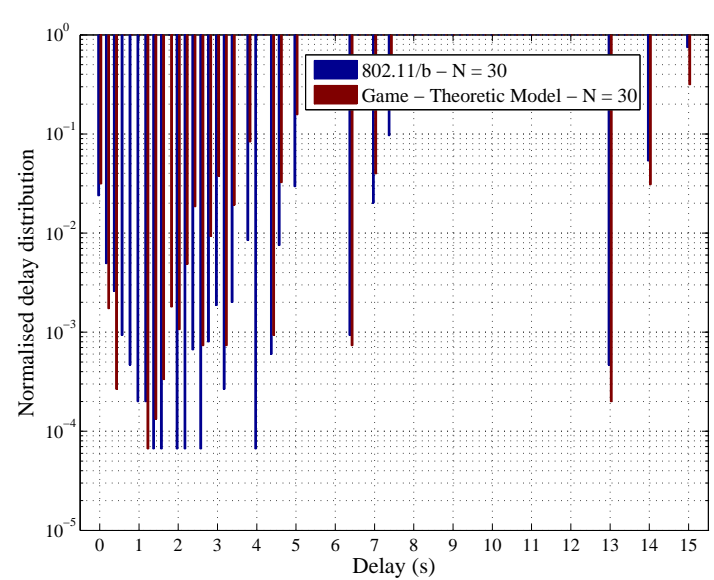

(f) Logarithmic scale

Figure B.14: Normalised delay distribution comparison for a network of 30 active nodes, operating in basic access method (DCF \& game-theoretic design) with 40-60 percent hidden terminal for each scenario. The bin-size is 0.2 seconds. 


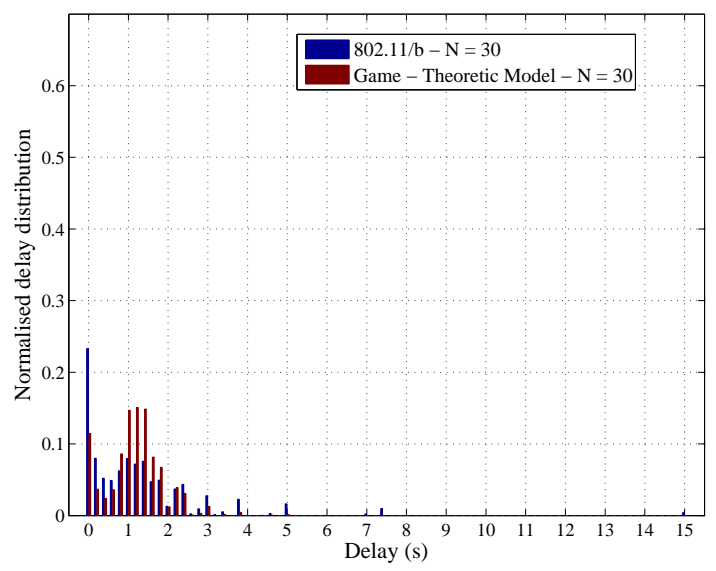

(a) Linear scale

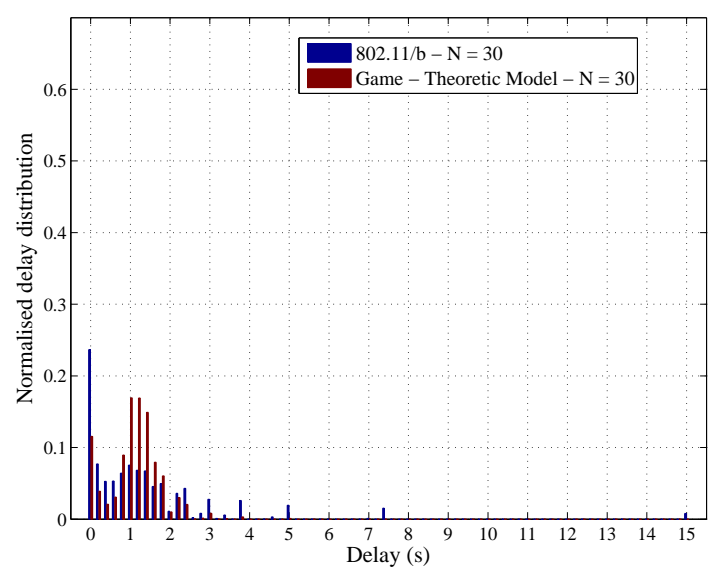

(c) Linear scale

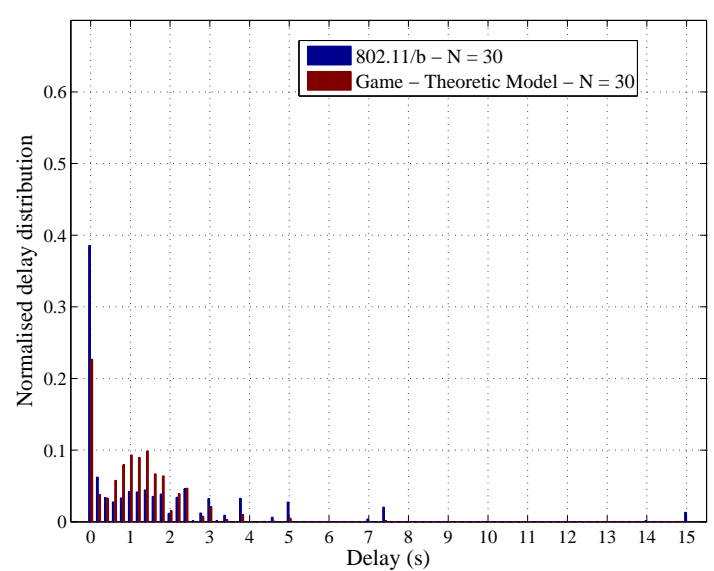

(e) Linear scale

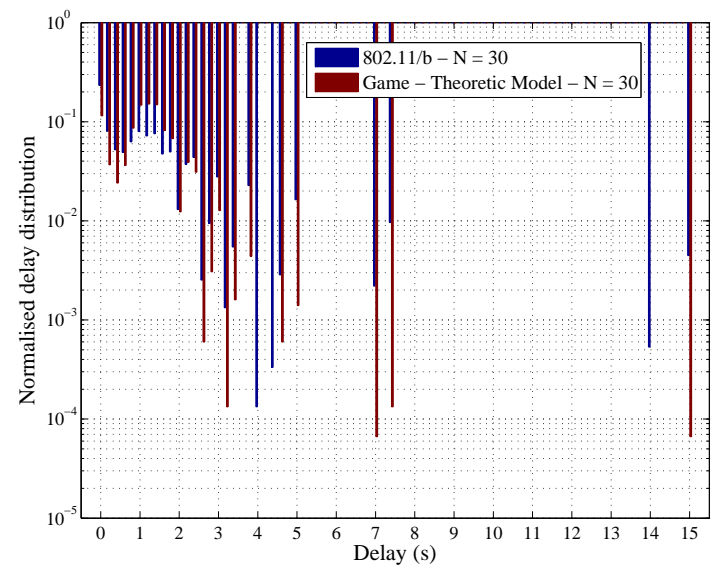

(b) Logarithmic scale

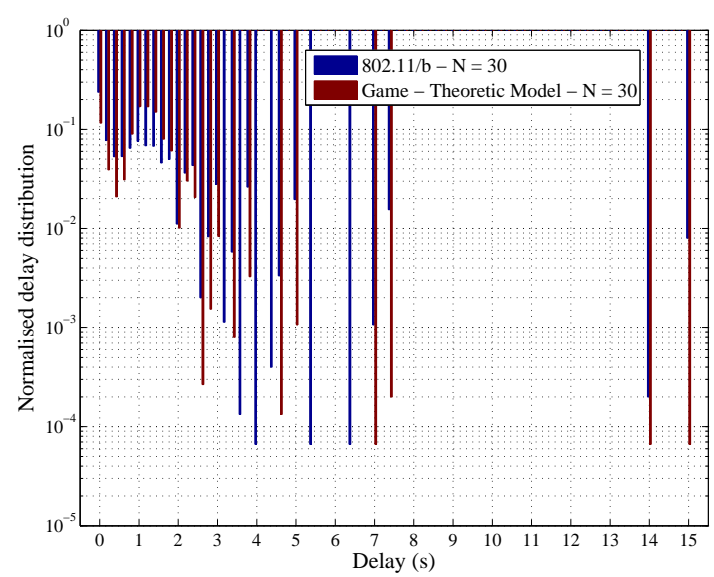

(d) Logarithmic scale

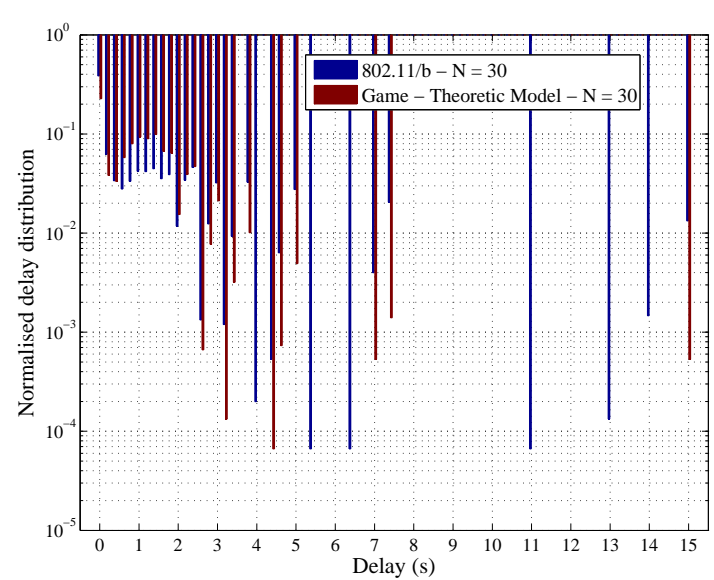

(f) Logarithmic scale

Figure B.15: Normalised delay distribution comparison for a network of 30 active nodes, operating in RTS/CTS access method (DCF \& game-theoretic design) with 10-30 percent hidden terminal for each scenario. The bin-size is 0.2 seconds. 


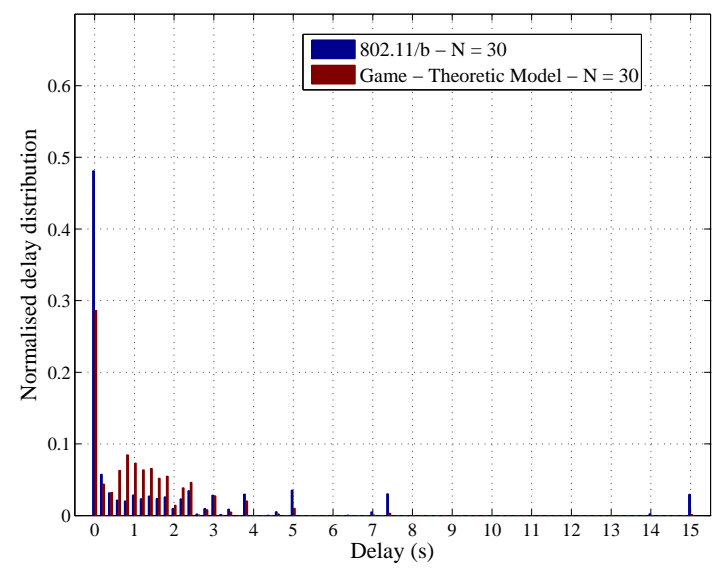

(a) Linear scale

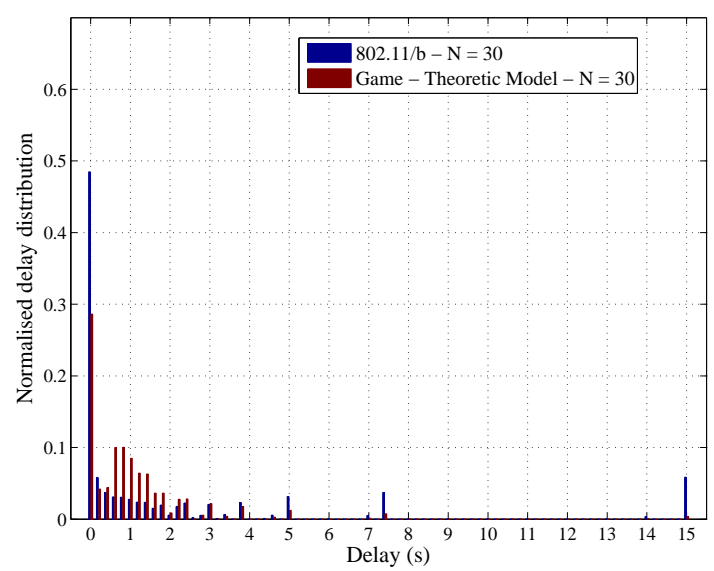

(c) Linear scale

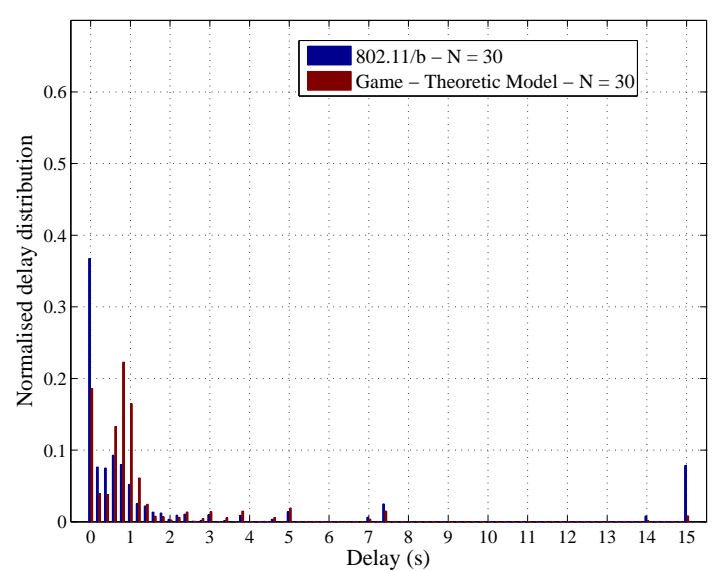

(e) Linear scale

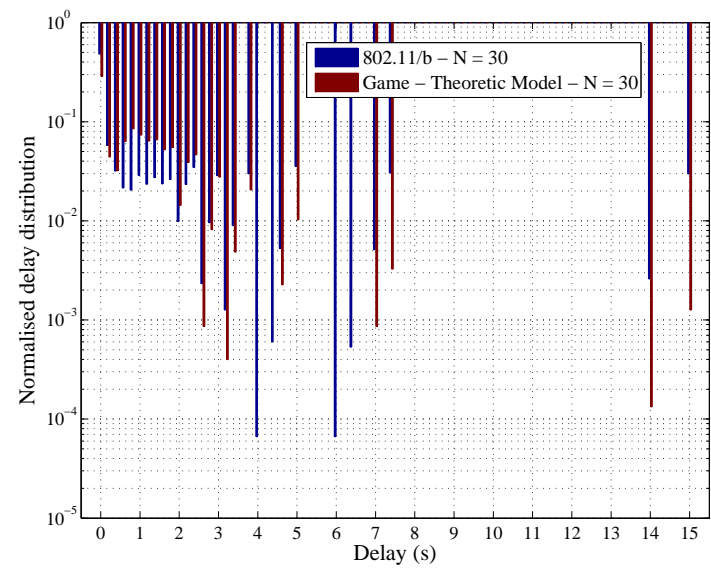

(b) Logarithmic scale

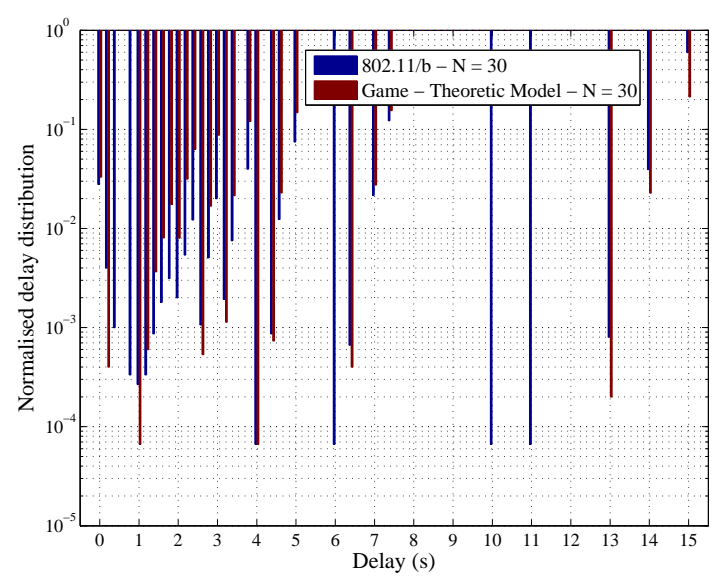

(d) Logarithmic scale

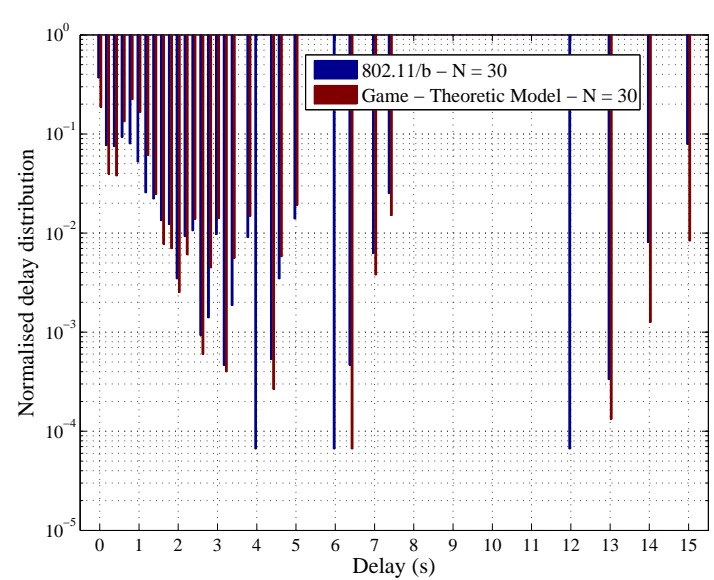

(f) Logarithmic scale

Figure B.16: Normalised delay distribution comparison for a network of 30 active nodes, operating in RTS/CTS access method (DCF \& game-theoretic design) with 40-60 percent hidden terminal for each scenario. The bin-size is 0.2 seconds. 


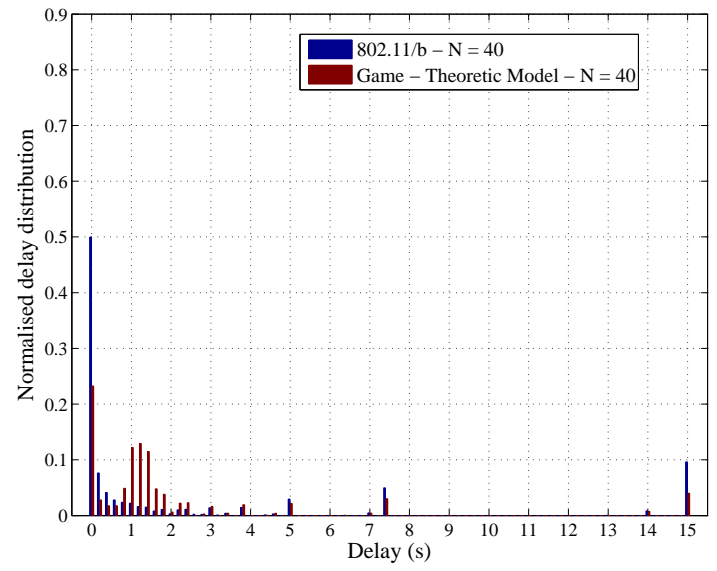

(a) Linear scale

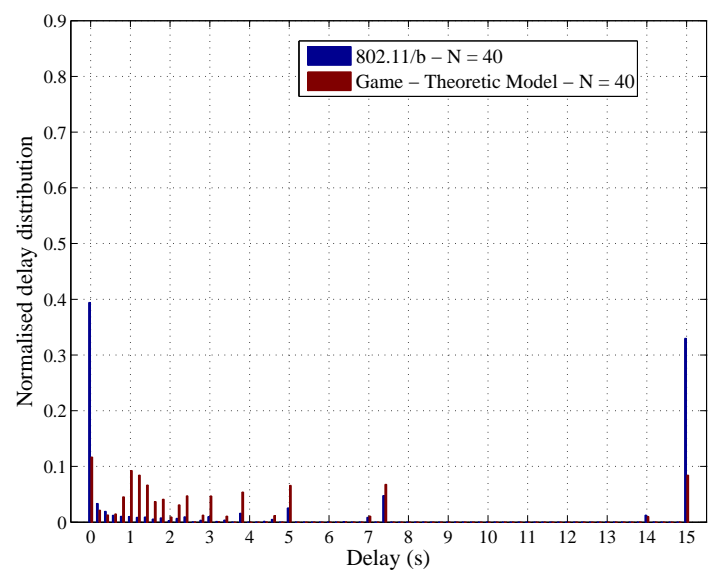

(c) Linear scale

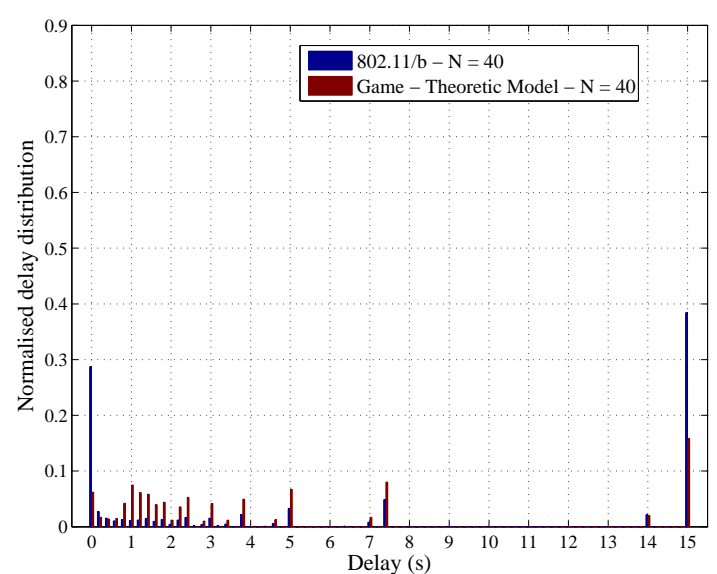

(e) Linear scale

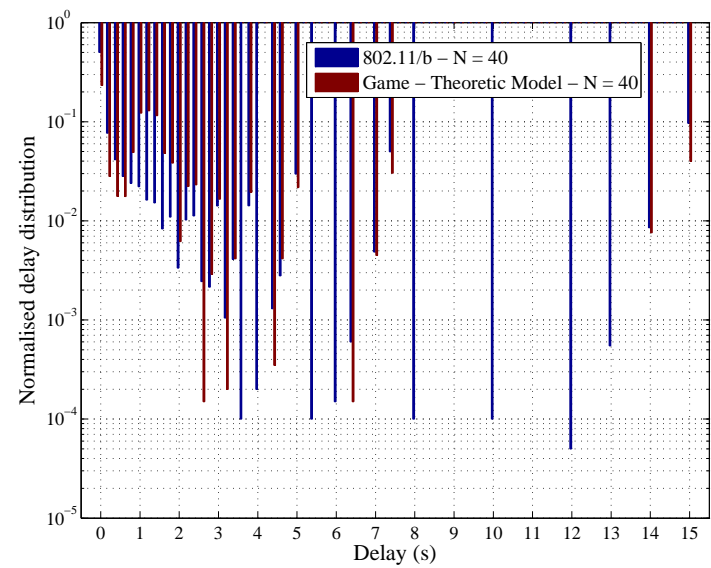

(b) Logarithmic scale

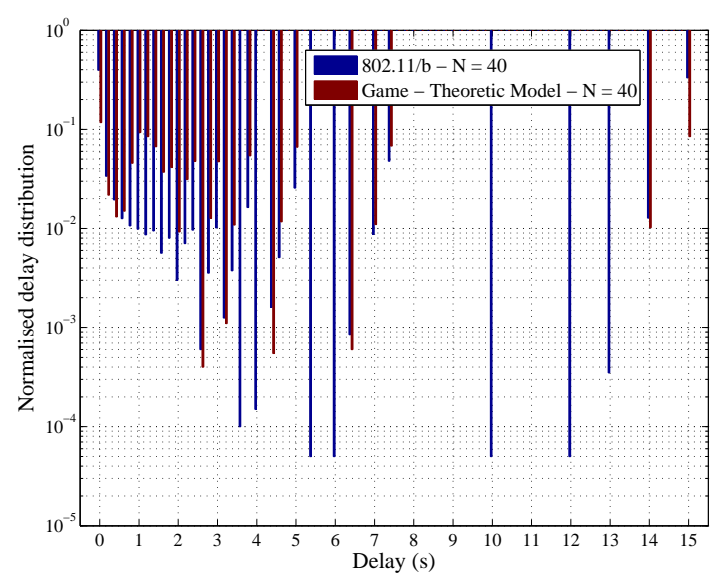

(d) Logarithmic scale

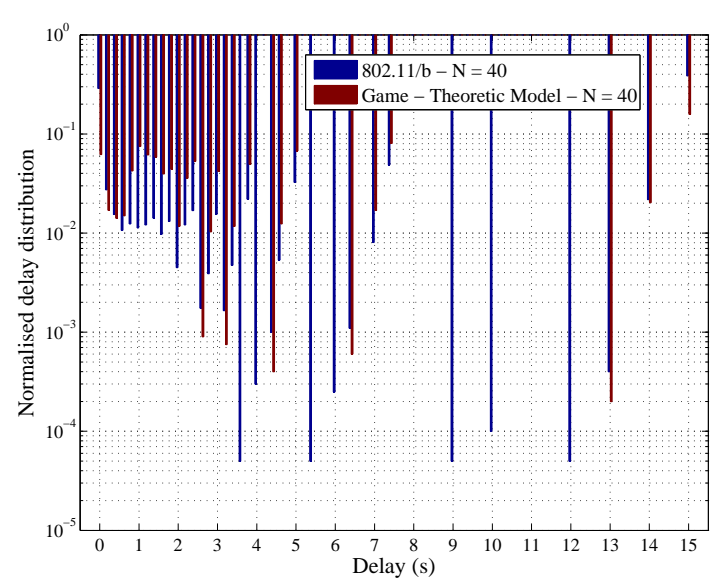

(f) Logarithmic scale

Figure B.17: Normalised delay distribution comparison for a network of 20 active nodes, operating in basic access method (DCF \& game-theoretic design) with 10-30 percent hidden terminal for each scenario. The bin-size is 0.2 seconds. 


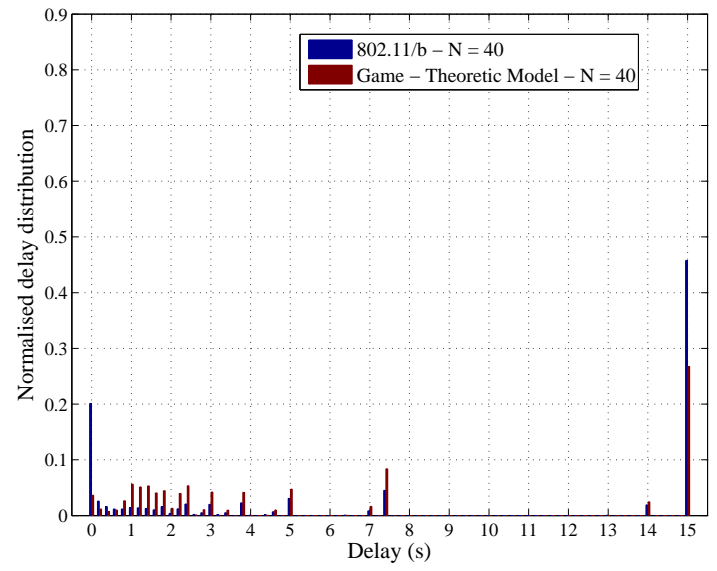

(a) Linear scale

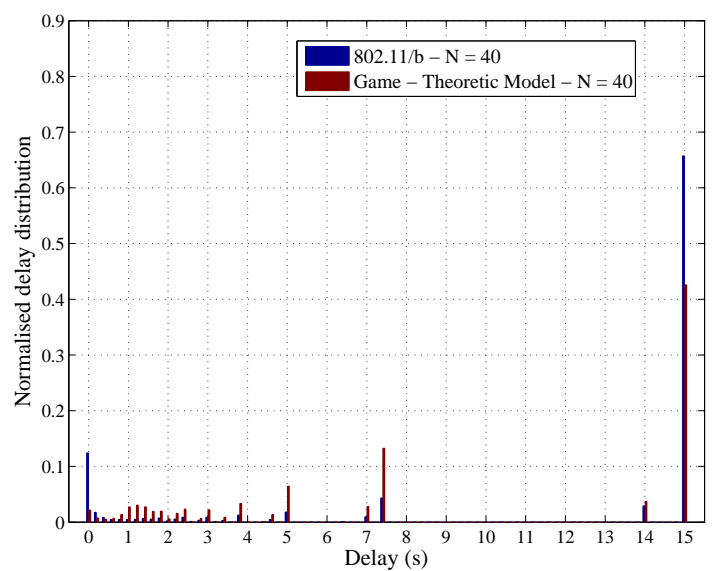

(c) Linear scale

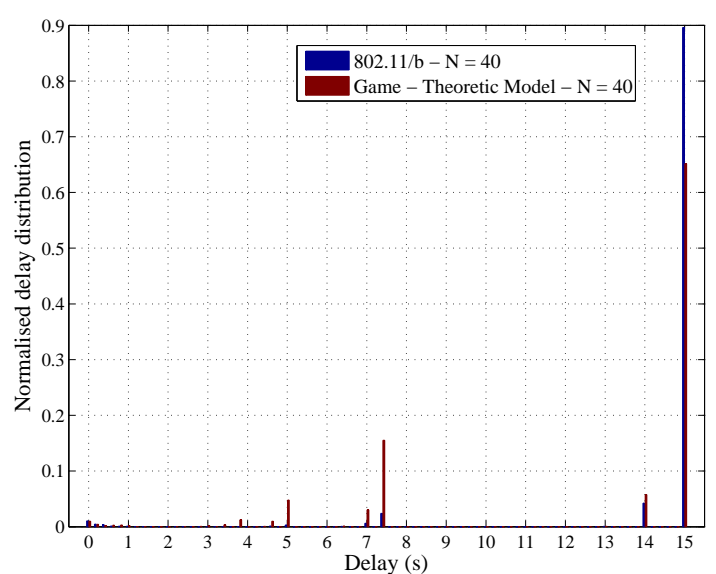

(e) Linear scale

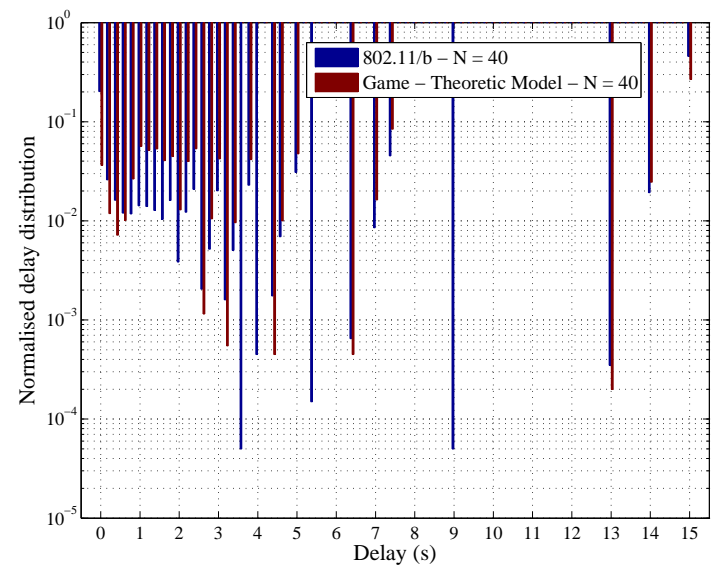

(b) Logarithmic scale

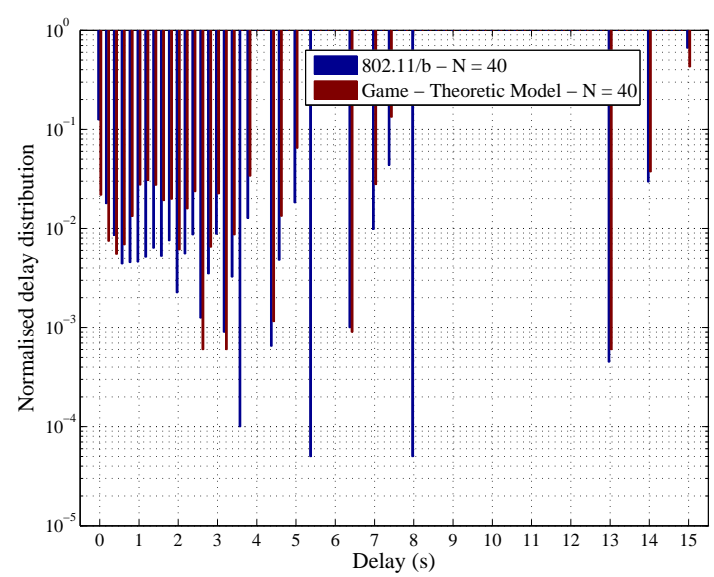

(d) Logarithmic scale

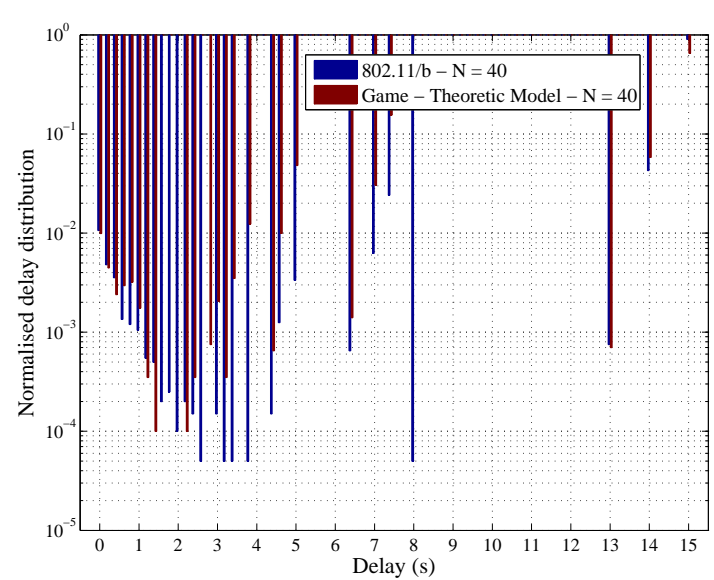

(f) Logarithmic scale

Figure B.18: Normalised delay distribution comparison for a network of 20 active nodes, operating in basic access method (DCF \& game-theoretic design) with 40-60 percent hidden terminal for each scenario. The bin-size is 0.2 seconds. 


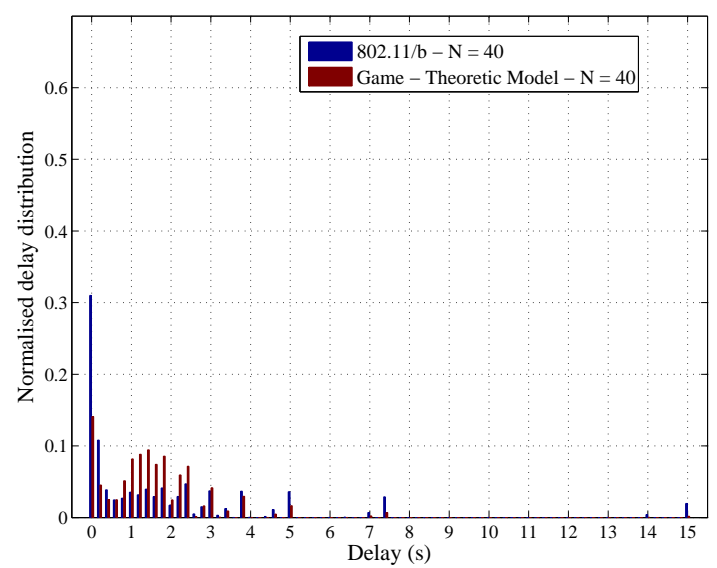

(a) Linear scale

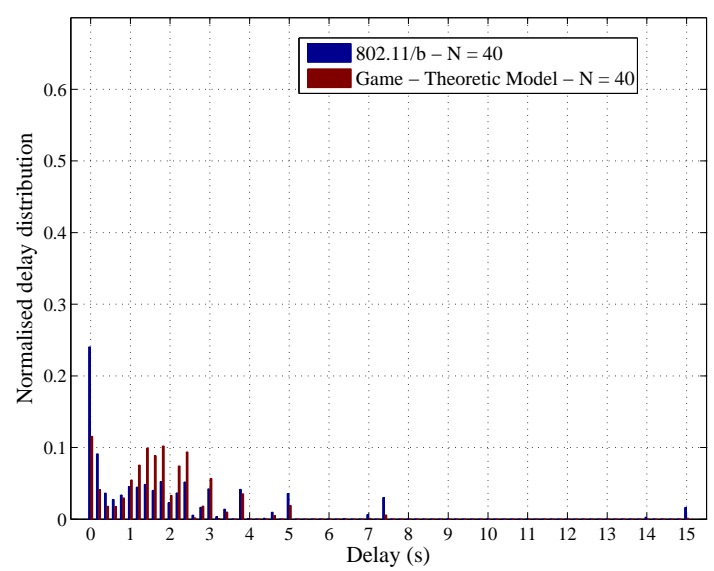

(c) Linear scale

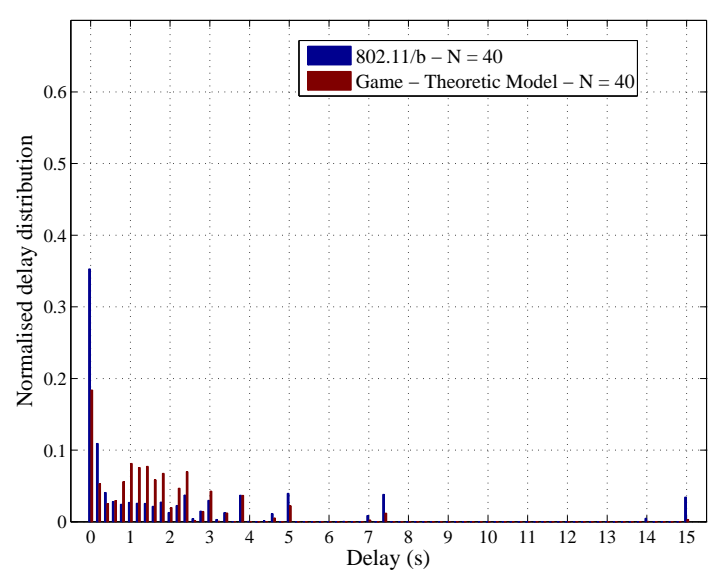

(e) Linear scale

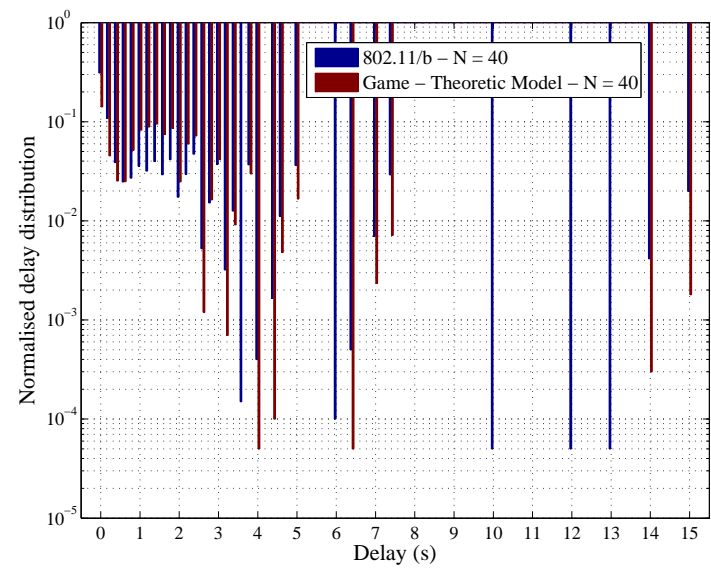

(b) Logarithmic scale

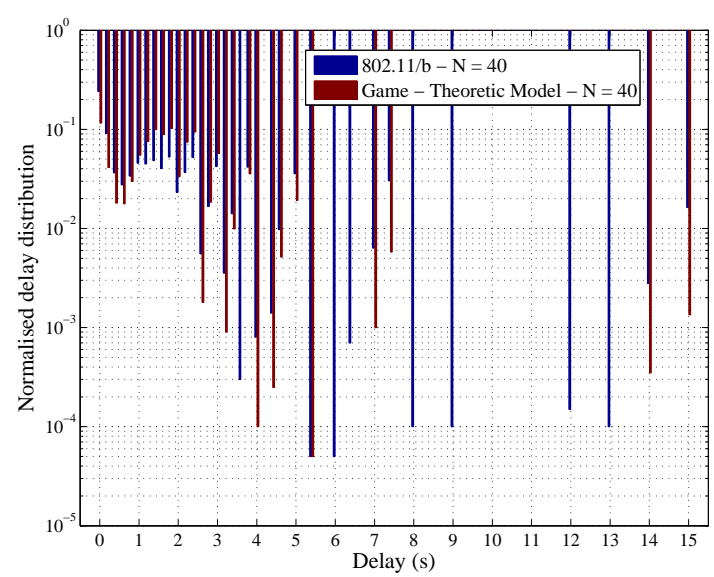

(d) Logarithmic scale

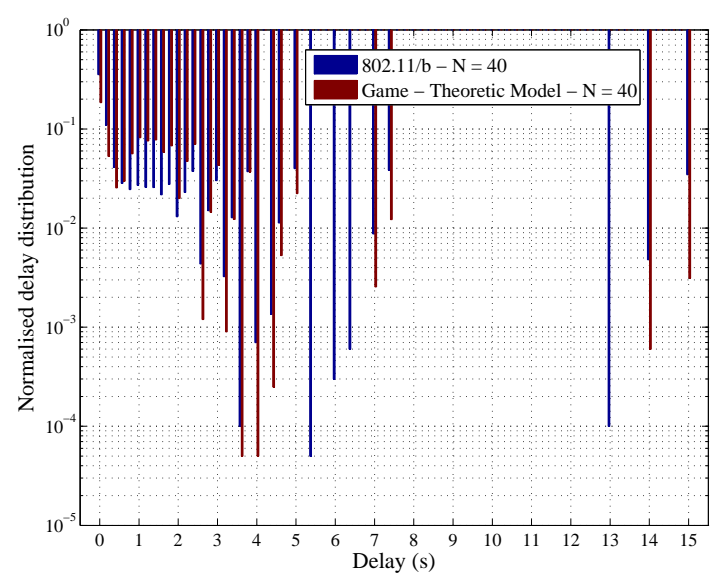

(f) Logarithmic scale

Figure B.19: Normalised delay distribution comparison for a network of 40 active nodes, operating in RTS/CTS access method (DCF \& game-theoretic design) with 10-30 percent hidden terminal for each scenario. The bin-size is 0.2 seconds. 


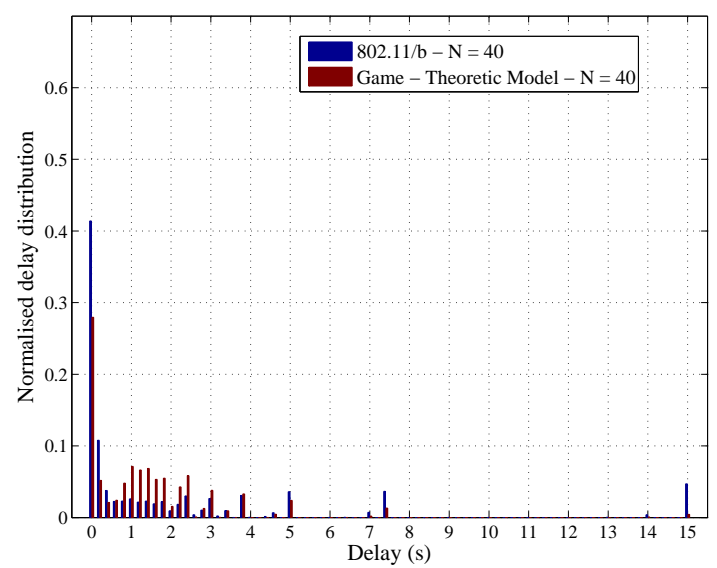

(a) Linear scale

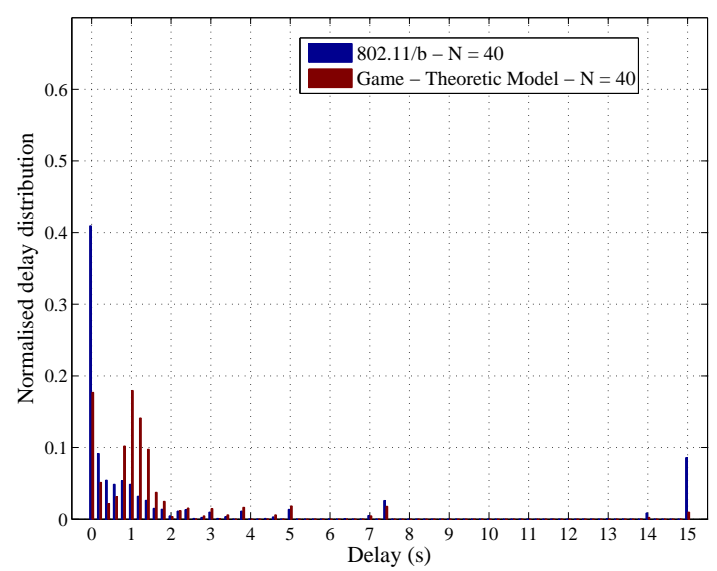

(c) Linear scale

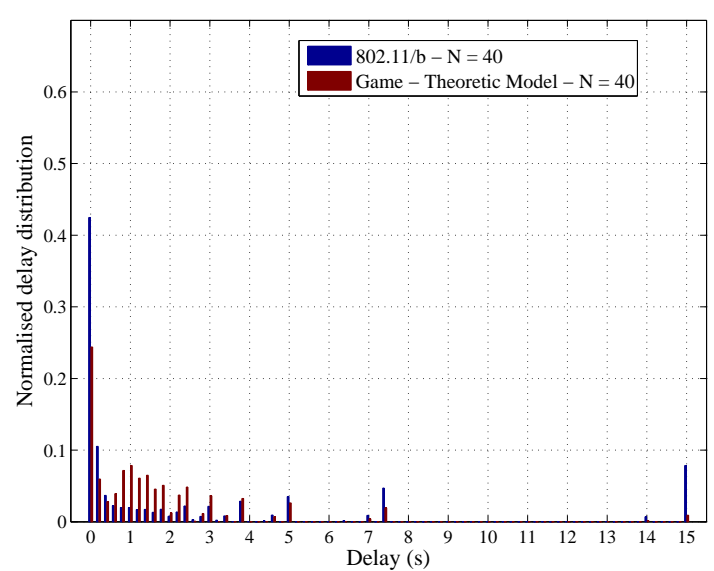

(e) Linear scale

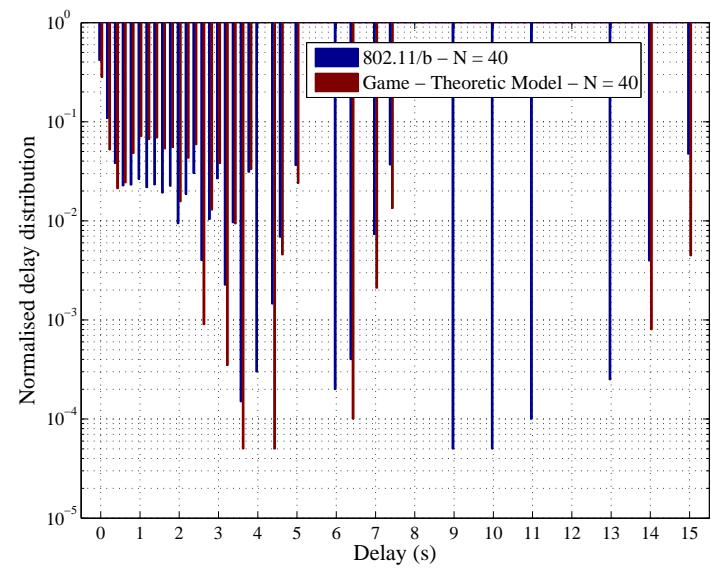

(b) Logarithmic scale

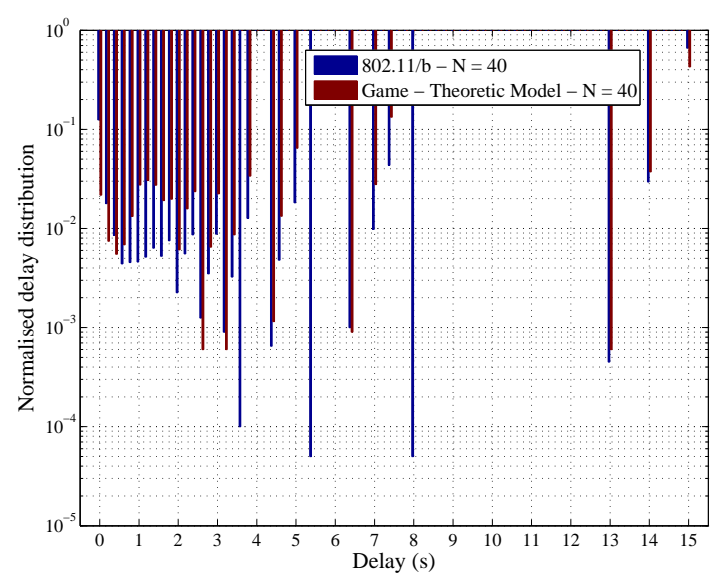

(d) Logarithmic scale

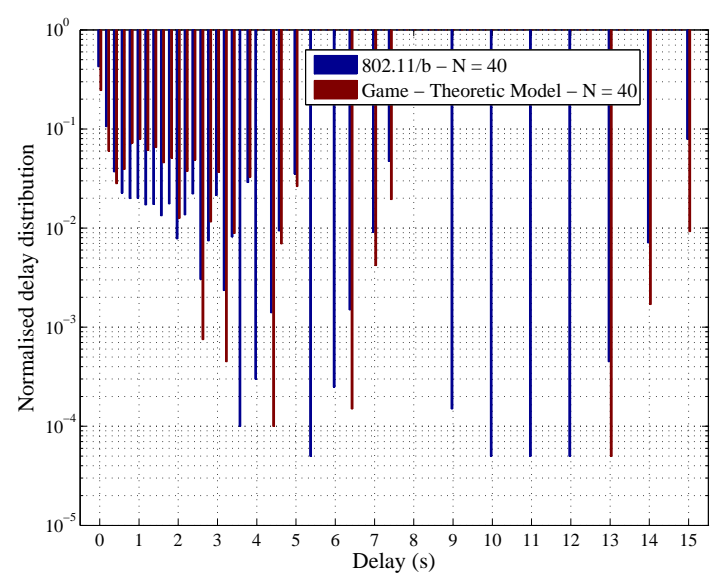

(f) Logarithmic scale

Figure B.20: Normalised delay distribution comparison for a network of 40 active nodes, operating in RTS/CTS access method (DCF \& game-theoretic design) with 40-60 percent hidden terminal for each scenario. The bin-size is 0.2 seconds. 


\section{APPENDIX C COMMON PARAMETERS USED FOR MiXIM- OMNETPP SimULATIONS}

\section{C.1 omnetpp.ini}

The omnetpp.ini are including of all the simulation configurations and is intended to be used with $802.11 / \mathrm{b}$ MAC. This simulation configuration also includes the scenario of 10 nodes with 10 percent hidden terminals

Listing C.1: Configuration Script code

$/ / *$ Modified by Hani Mortazavi

//* copyright: (C) 2008 Telecommunication Networks Group (TKN) at

$/ / * \quad$ Technische Universitaet Berlin, Germany.

\section{[General]}

cmdenv-express-mode $=$ true

network $=$ MyNetVer2

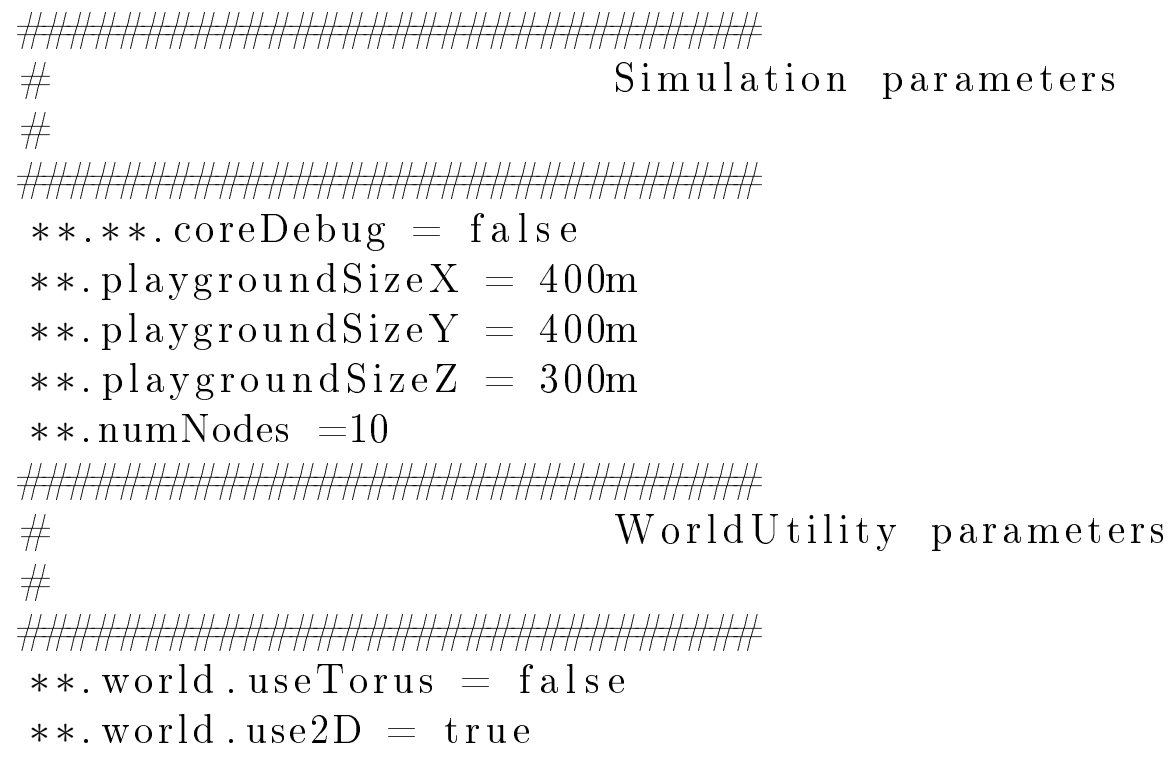




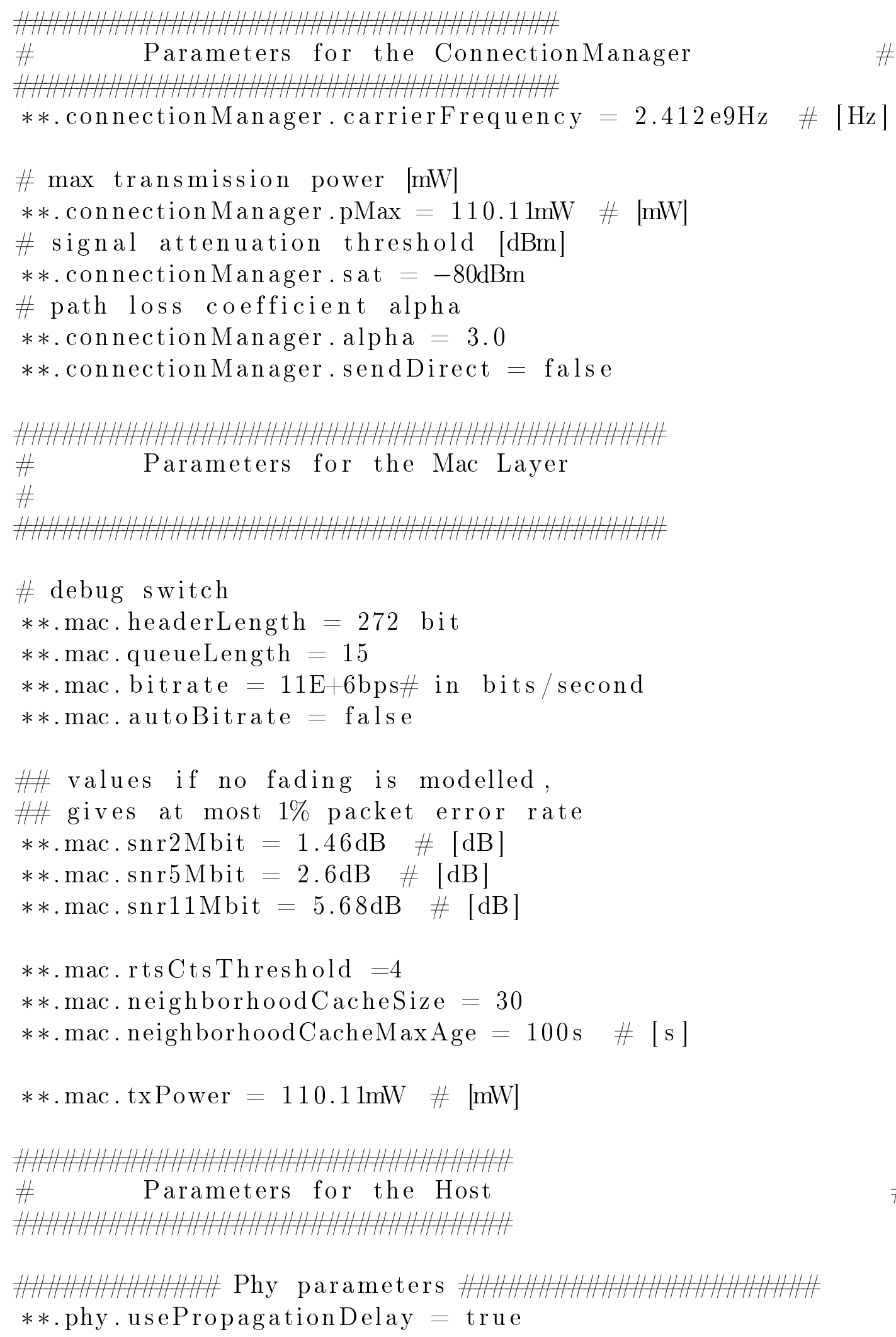




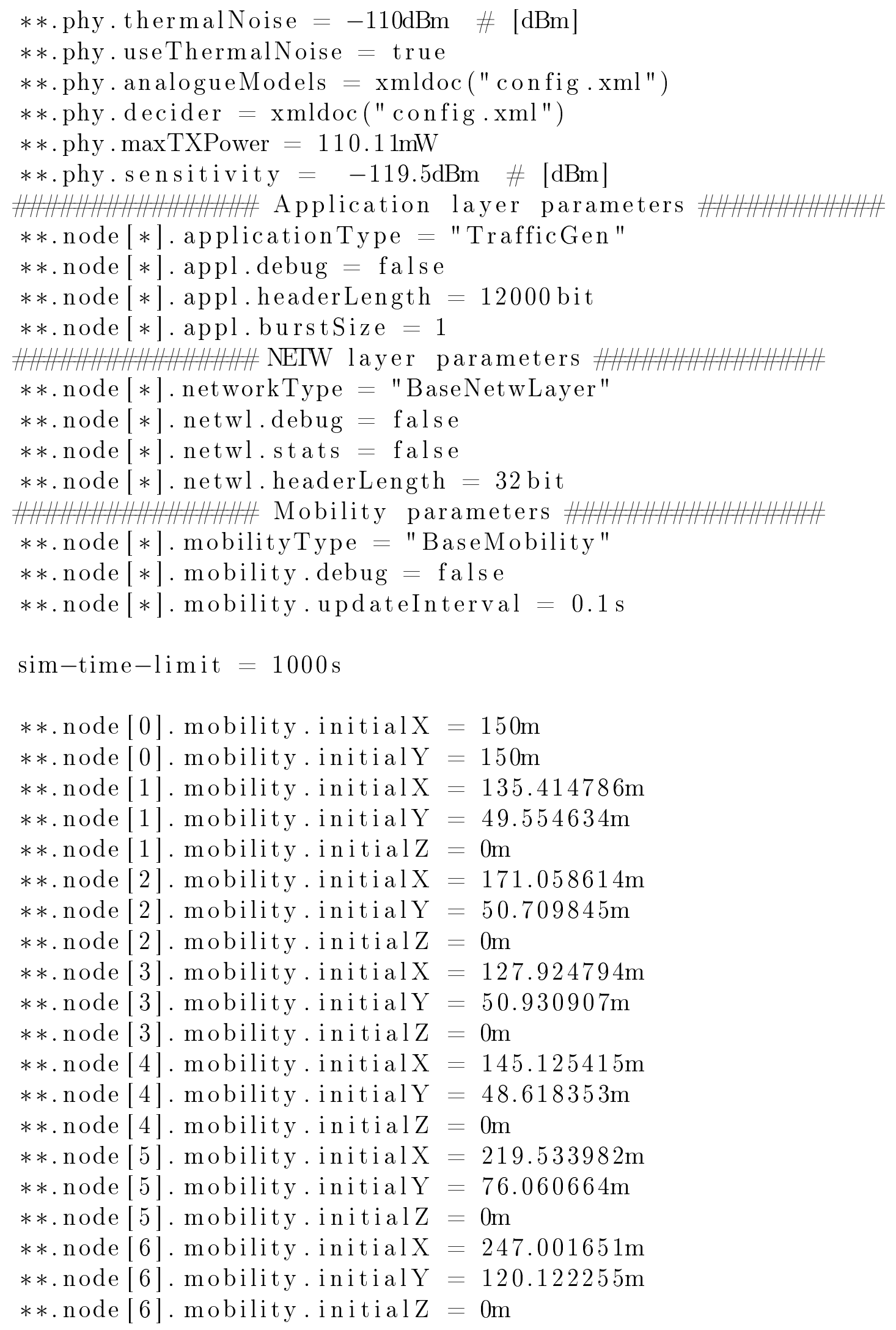


$* *$. node [7]. mobility initialX $=236.183631 \mathrm{~m}$

$* *$. node [7]. mobility initialY $=96.386739 \mathrm{~m}$

$* *$. node [7]. mobility initialZ $=0 \mathrm{~m}$

$* *$. node [8]. mobility initialX $=204.365795 \mathrm{~m}$

$* *$. node [8]. mobility initialY $=64.289088 \mathrm{~m}$

$* *$. node $[8]$. mobility initialZ $=0 \mathrm{~m}$

$* *$. node $[9]$. mobility initialX $=231.965451 \mathrm{~m}$

$* *$. node [9]. mobility initialY $=90.136280 \mathrm{~m}$

$* *$. node $[9]$. mobility . initial $\mathrm{Z}=0 \mathrm{~m}$

\#Hidden Covered

\# $3 \quad 6$

\# $1 \quad 8$

\# $3 \quad 6$

\# $20 \quad 7$

\# $0 \quad 9$

\# 45

\# $3 \quad 6$

\# $0 \quad 9$

$\begin{array}{lll}\text { \# } & 2 & 7\end{array}$

\# $0 \quad 9$

$\# \mathrm{f}_{\text {_res_ }} \mathrm{h}=0.20$

\#f_res_c $=0.80$ 


\section{APPENDIX D PUBLICATIONS}

S.H.E.M. Najafabadi and C.C. Constantinou. Game theoretic approach to medium access control in wireless networks. In Wireless Communications and Networking Conference (WCNC), 2013 IEEE, pages 872-877, April 2013.

S.H.E.M. Najafabadi and C.C. Constantinou. A game theoretic model for wireless medium access control in the presence of hidden terminals. In 2013 IEEE 24th International Symposium on Personal, Indoor and Mobile Radio Communications (PIMRC) 2013, pages 1741-1746, London, United Kingdom, September 2013. 